\title{
GAS-EXPANDED LUBRICANTS FOR INCREASED ENERGY EFFICIENCY AND CONTROL IN ROTATING MACHINERY
}

\author{
A Dissertation \\ Presented to \\ the faculty of the School of Engineering and Applied Science \\ University of Virginia \\ in partial fulfillment \\ of the requirements for the degree \\ Doctor of Philosophy
}

by

Brian Keith Weaver

December

2014 
APPROVAL SHEET

The dissertation

is submitted in partial fulfillment of the requirements

for the degree of

Doctor of Philosophy

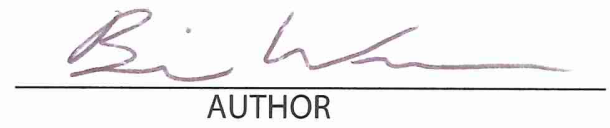

The dissertation has been read and approved by the examining committee:

\begin{tabular}{c} 
Andres Clarens \\
\hline Advisor \\
Lisa Colosi Peterson \\
\hline Houston Wood \\
\hline Alexandrina Untaroiu \\
\hline Christopher Goyne \\
\hline
\end{tabular}

Accepted for the School of Engineering and Applied Science:

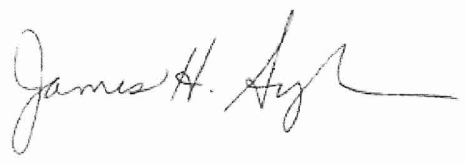

Dean, School of Engineering and Applied Science

December

2014 


\begin{abstract}
Bearing lubricants are essential to the efficient and reliable operation of high-speed rotating machinery. Lubricant films separate the rotating shaft and the stationary bearing structure to minimize friction and remove heat while providing stiffness and damping forces to the shaft that enable its smooth operation at high speeds. As the demand for larger, faster machines increases along with the need to push existing equipment to new extremes of operation, the need for lubrication technologies that can support these higher demands is also increased. However, the difficulty in this need lies in the fact that bearing lubricants introduce inefficiencies and other performance limitations of their own. The goal of this work is to develop a novel lubrication technology called gas-expanded lubricants (GELs) which stand to provide real-time control over bearing and rotor dynamics while increasing bearing efficiency and reliability, and without comprising the other important functions that lubricants are needed to provide. GELs are controlled by dissolving carbon dioxide into synthetic lubricants at high pressure. The control of mixture composition provides direct control over the lubricant viscosity. Larger machines often require the use of more viscous fluids to support the weight of the rotor, which can then lead to higher bearing losses and heat generation due to the viscous shear of the fluid. Greater lubricant viscosities also tend to increase bearing stiffness which in many cases can be detrimental to the rotordynamic stability of the machine. The tunability that these fluids impart can be used prior to machine startup or shutdown to maximize
\end{abstract}


rotordynamic stability or to maximize bearing efficiency and reduce operating temperatures during steady operation, thereby increasing the life of the bearing. GELs also provide the ability to adapt to dynamic operating conditions as a result of changing environmental or loading conditions. Other lubricant and bearing technologies have been developed in attempts to improve machine performance, but none of them offer the realtime control of bearing and rotor dynamics of GELs without comprising other important bearing properties such as load capacity.

To fully understand the implications of this lubricant technology on bearing and overall machine performance, it was necessary to develop a fundamental understanding of both the properties of these fluids as well as their expected impacts on machine performance and design. This work applied a combination of experimental and modeling techniques to answer questions related to the development and use of GELs in rotating machinery. The viscosity, diffusivity, and thermal properties of GELs were measured and combined with existing data in the literature to fully characterize their behavior. The mixture viscosity was found to be driven by the amount of carbon dioxide dissolved into the fluid while effects on other properties were found to be minor. This knowledge allowed for the accurate prediction of GEL performance in a variety of machinery bearings operating at a range of loads and speeds. It was found that the changes in lubricant viscosity can significantly affect bearing efficiency, operating temperature, and stiffness and damping forces while keeping other bearing metrics such as eccentricity and minimum film thickness within acceptable ranges. The effects on bearing dynamics were also found to have significant impacts on machine rotordynamic performance metrics including rotordynamic stability, though effects on unbalance-driven vibration 
amplitudes of the rotor were found to be minor. A number of unique design considerations are discussed in the context of two experimental test rigs that were designed and assembled for the characterization of GEL performance in high-pressure bearings and seals. This work provides the foundation for a novel lubrication technology with the potential to significantly increase performance and control in high-speed rotating machinery. 


\section{Acknowledgements}

This work would not have been possible without the support of many people and organizations. First, I must thank my wife Janelle for her love, encouragement, and support throughout my time at UVA. We have endured both the joys and pains of graduate school together, and without her early encouragement and constant support I would have never taken on such a great challenge. I must also thank my family for their never-ending support, always encouraging me to keep my head up and putting up with my crazy schedule.

I would also like to thank my advisor, Dr. Andres Clarens. His expertise, open mind, and scientific drive are unparalleled and I believe the University of Virginia is lucky to have such talent amongst their ranks. I am honored to say that I have been able to work with him these past four years, learning not only the subject of my research but also learning how to better research new ideas, think critically, and present those ideas in a scientifically and logically sound manner. Many thanks are also extended to the rest of my dissertation committee for their feedback throughout this process.

Much gratitude is also extended to the professors, professional researchers, and graduate students in ROMAC. They have been an excellent group of people to work with throughout the duration of this project and this work would not have been possible without their collaborative support. Special thanks are extended to Alexandrina Untaroiu, Roger Fittro, Houston Wood, Chris Goyne, Bob Rockwell, Jason Kaplan, Patrick Migliorini, Neal Morgan, Brad Nichols, and Ya Zhang for their support of the modeling 
and test rig efforts performed in this work. Thanks are also extended to former research staff Amir Younan and Timothy Dimond for their guidance and teaching in my early days of studying mechanical engineering. I believe your efforts to convert me into a mechanical engineer have been successful!

Many thanks are also reserved for my lab mates past and present in the VestLab. Thank you Shibo Wang, Bo Liang, Zhiyuan Tao, Buddy Wilkins, Lyu Xiaotong, Xiaowei Liu, Eleazer Resurreccion, and Alec Gosse for your friendship, advice, and feedback throughout my time at UVA.

Support from the U.S. National Science Foundation and the Department of Commerce must also be acknowledged as this work would not have been made possible without their support for the discovery and exploration of new technologies. 


\section{Contents}

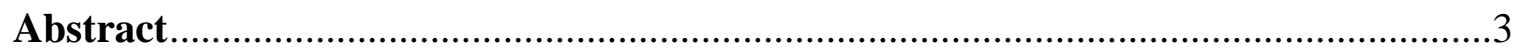

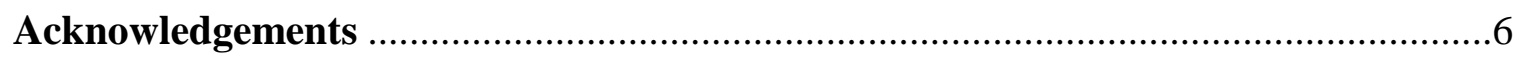

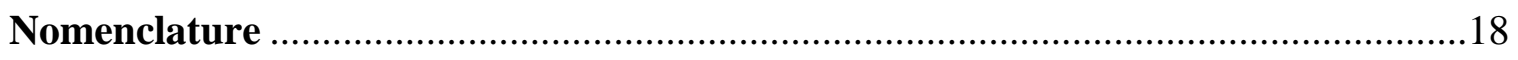

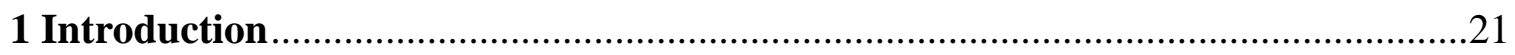

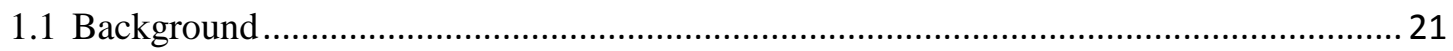

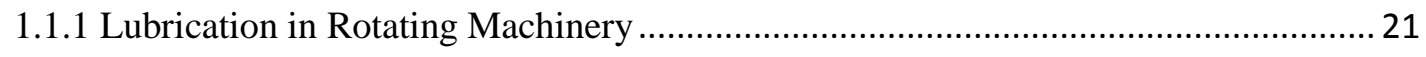

1.1.2 Properties of Lubricant-Carbon Dioxide Mixtures ……………………………....... 26

1.1.3 Effects of Dissolved Solutes on Lubricant Performance............................................ 29

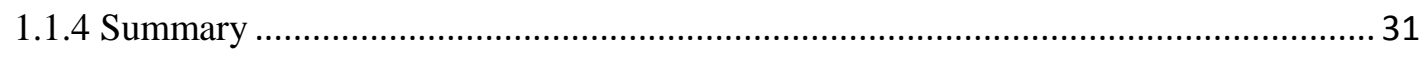

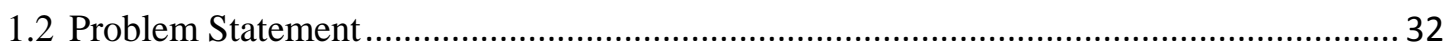

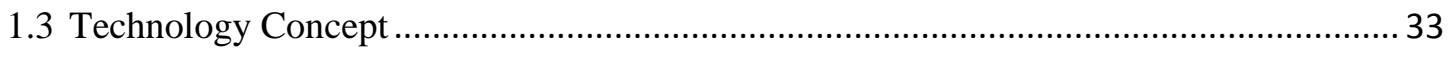

1.4 Research Objectives, Scope, and Overview of Dissertation ............................................ 35

\section{Properties and Performance of Gas-Expanded Lubricants in Tilting Pad Journal}

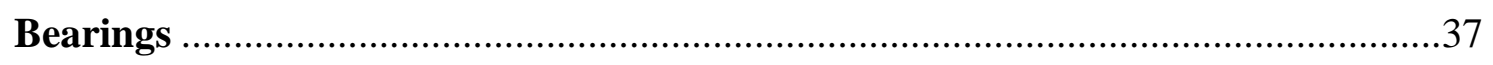

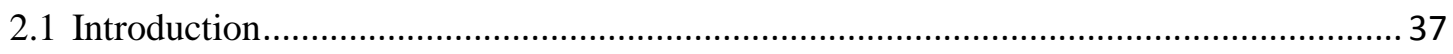

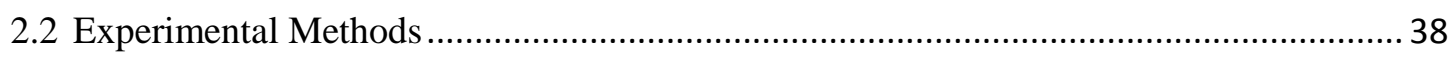




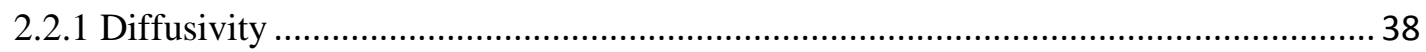

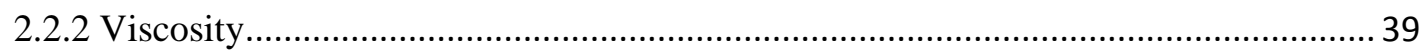

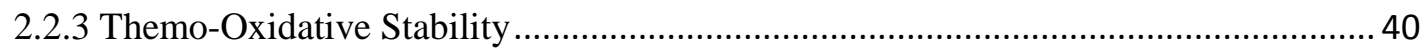

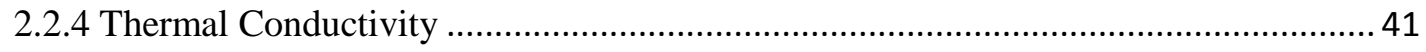

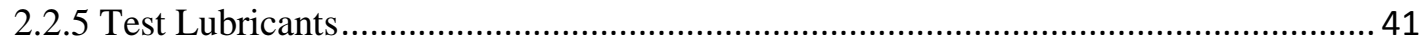

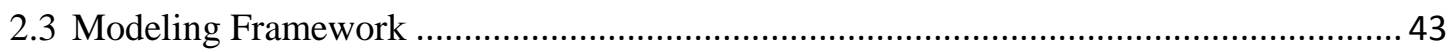

2.3.1 Solubility of Carbon Dioxide in Lubricant …..................................................... 43

2.3.2 Thermoelastohydrodynamic Bearing Model........................................................ 44

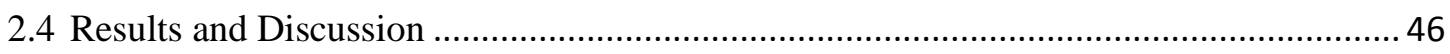

2.4.1 Gas-Expanded Lubricant Properties..................................................................... 46

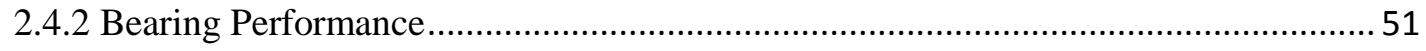

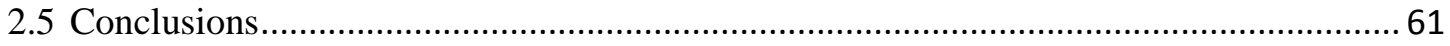

\section{Gas-Expanded Lubricant Performance and Effects on Rotor Stability in}

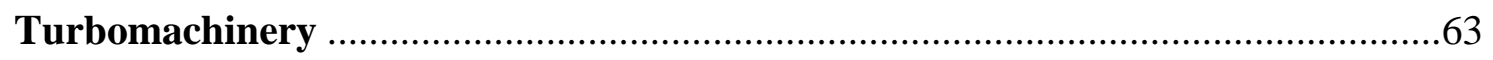

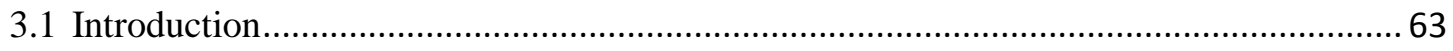

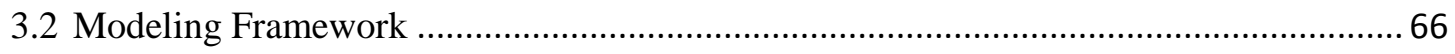

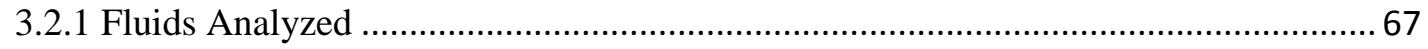

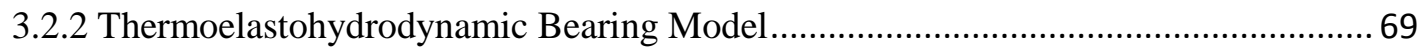

3.2.3 Finite Element Models of Rotordynamic Performance............................................ 71

3.2.4 Transient Finite Element Model of Rotordynamic Performance ............................... 74

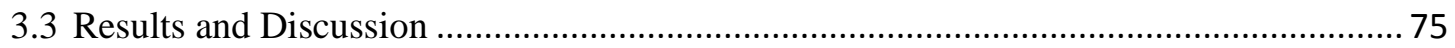




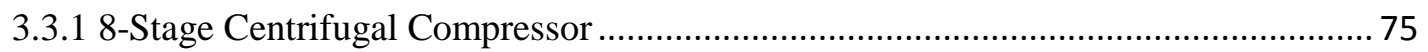

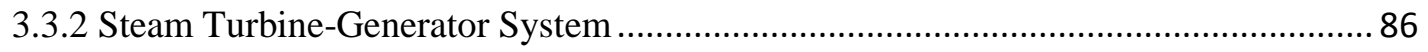

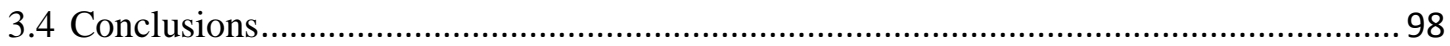

4 Nonlinear Analysis of Rub Impact in a Three-Disk Rotor and Correction via

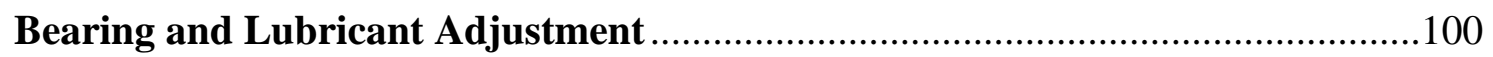

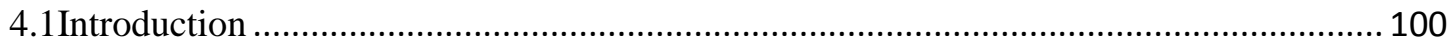

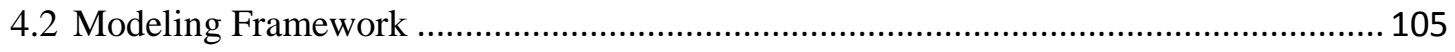

4.2.1 Thermoelastohydrodynamic Bearing Model ........................................................ 105

4.2.2 Steady-State Finite Element Rotordynamic Model............................................... 107

4.2.3 Nonlinear Finite Element Rotordynamic Model ................................................. 108

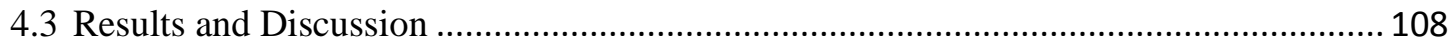

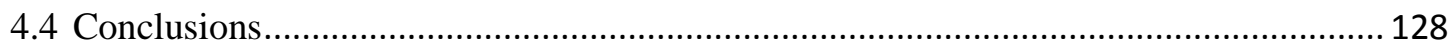

5 Design of a Gas-Expanded Lubricants Bearing Test Rig..................................130

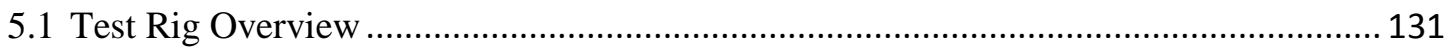

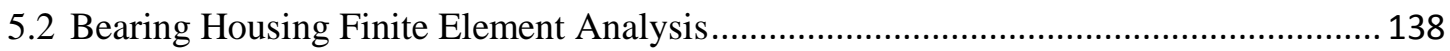

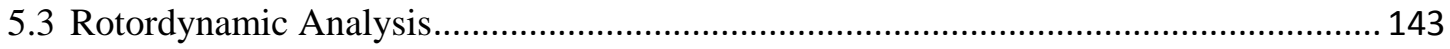

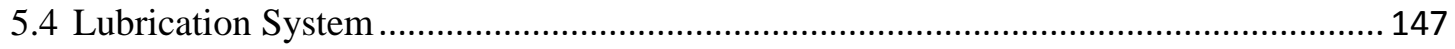

5.5 Data Acquisition and Instrumentation ..................................................................... 150

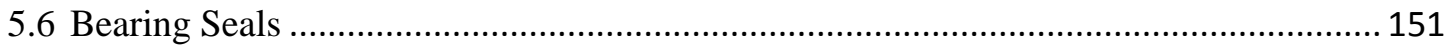

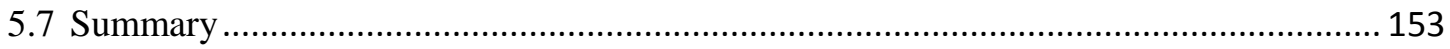




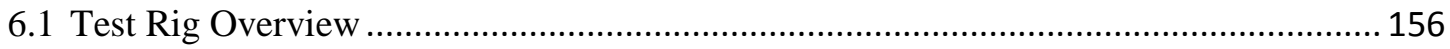

6.2 Seal Design and Performance Analysis .................................................................. 159

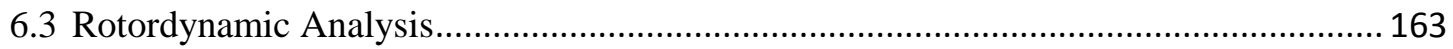

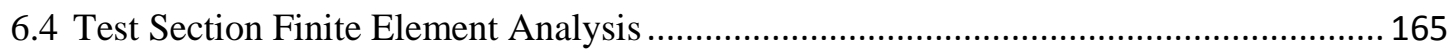

6.5 Base Modal Analysis ………………………………………………………….... 169

6.6 Lubrication System Modifications............................................................................... 170

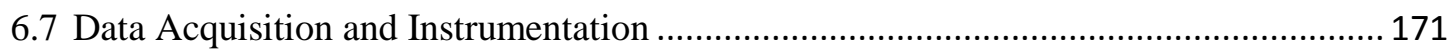

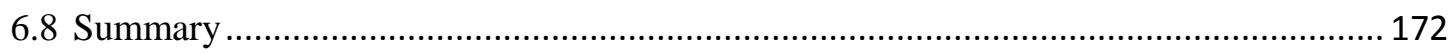

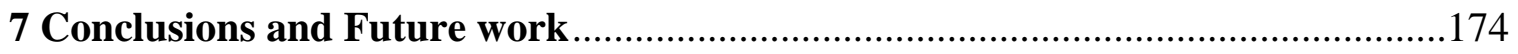

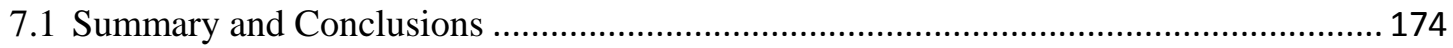

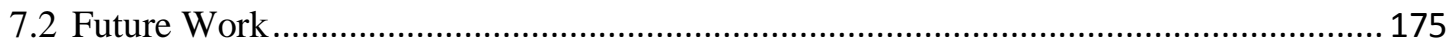

7.2.1 Seal Testing and Model Validation................................................................... 176

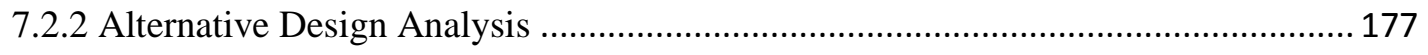

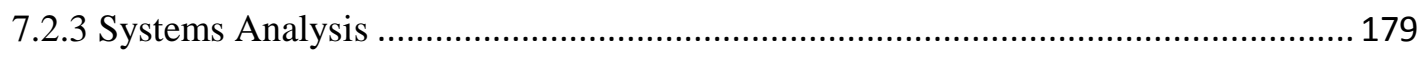

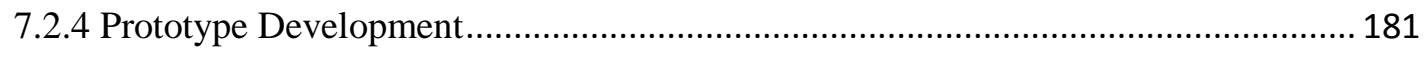

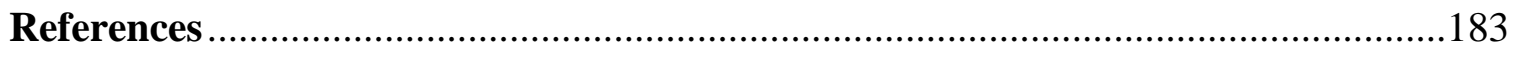




\section{List of Figures}

1.1 Lubricant shear in the non-supporting regions of bearings results in excessive power loss and higher operating temperatures 24

1.2 Lubricant viscosity at two isotherms as a function of dissolved $\mathrm{CO}_{2}$ mass fraction....... 28

1.3 Lubricant density remains relatively unchanged for a wide range of pressures and temperatures

1.4 Power losses increase drastically as the flow regime transitions from laminar to turbulent flow .33

1.5 Gas-expanded lubricants provide real-time control over lubricant viscosity

2.1 High-pressure fluid-phase stability curves for POE and carbon dioxide. GELs form above the isotherms; below them multiple phases exist. Experimental data are from Hauk and model results are from this work

2.2 Diffusion coefficients as a function of lubricant viscosity. The fitted line has a slope of -0.535 , a relationship found comparable to others reported

2.3 GEL viscosity as a function of mixture composition as (a) $40^{\circ} \mathrm{C}$ and (b) $100^{\circ} \mathrm{C}$

2.4 Thermo-oxidation of lubricant- $\mathrm{CO}_{2}$ mixtures does not appear to influence the long-term stability of the lubricants compared to lubricants alone

2.5 Pressure (solid lines) and temperature (dashed lines) distributions on the loaded pad \#4 of the tilting pad journal bearing. Profiles are shown for (a) POE and (b) the POE-based GEL at $200 \mathrm{rpm}$ .53 
2.6 Pressure (solid lines) and temperature (dashed lines) distributions on the loaded pad \#4 of the tilting pad journal bearing. Profiles are shown for (a) POE and (b) the POE-based GEL at 20,000 rpm. .54

2.7 GEL composition in the fluid film region along pad \#4 of the tilting pad journal bearing at (a) $200 \mathrm{rpm}$ and (b) 20,000 rpm .56

2.8 Power loss as a function of speed .58

2.9 Journal eccentricity ratio as a function of speed .59

2.10 Synchronously reduced stiffness and damping coefficients as a function of speed

2.11 Minimum film thickness as a function of speed. 61

3.1 Finite element model of the 8-stage compressor. .71

3.2 Finite element model of the steam turbine-generator system: a. generator, b. low-speed pinion, c. high-speed pinion, d. flexible coupling, and e. steam turbine 72

3.3 Reynolds numbers for Pad 2 in the compressor bearings .75

3.4 Compressor bearing power loss as a function of speed. .76

3.5 Minimum film thickness as a function of speed. .77

3.6 Journal eccentricity ratio as a function of speed .78

3.7 Critical speed map of the centrifugal compressor .78

3.8 Unbalance response of the first bending mode 80

3.9 Compressor stability margin 80

3.10 Dynamic stiffness (a) and damping (b) coefficients for the compressor bearings 81

3.11 Direct a. stiffness and b. damping coefficients as a function of lubricant viscosity 83

3.12 Cross-coupled stiffness coefficients as a function of lubricant viscosity .84 
3.13 Transient $\mathrm{x}$ and $\mathrm{y}$ displacments of the left compressor bearing 86

3.14 Power loss as a function of speed in the high-speed pinion bearings .88

3.15 Reduced operating temperatures in the high-speed pinion GEL case have significant effects on a. fluid film thickness and b. eccentricity ratio. .90

3.16 Reynolds numbers for Pad 2 in the coupling-end turbine bearing..... 91

3.17 Power loss as a function of speed in the coupling-end turbine bearing shows the onset of turbulent flow at $10,000 \mathrm{rpm}$ 92

3.18 Mode 5 of the generator and low-speed pinion for the a. GEL and b. ISO fluid cases... 95

3.19 Unbalance response of the turbine first bending mode 97

3.20 Steam turbine stability margin 97

4.1 Finite element model of the 3-disk rotor 107

4.2 Critical speed map of the 3-disk rotor.

4.3 Center disk displacements for the 0.2 preload and load-between-pad bearing configurations

4.4 Dynamic stiffness (a) and damping (b) coefficients for cases 2, 6, 10, and 14. 113

4.5 Bifurcation diagrams for cases 2 (a) and 14 (b) 115

4.6 Shaft whirl orbits for cases 2 (a) and 14 (b) 116

4.7 Spectrum analysis of cases 2 (a) and 14 (b) 117

4.8 Center disk displacements for the 0.2 preload and load-on-pad bearing configuration 119

4.9 Dynamic stiffness (a) and damping (b) coefficients for cases 1, 5, 9, and 13 120

4.10 Center disk displacements for the POE lubricant and the $50.8 \mu \mathrm{m}$ bearing clearance.. 122 4.11 Dynamic stiffness (a) and damping (b) coefficients for cases 1, 2, 3, and 4 123 
4.12 Center disk displacements for the POE lubricant and $25.4 \mu \mathrm{m}$ bearing clearance.

4.13 Dynamic stiffness (a) and damping (b) coefficients for cases 5, 6, 7, and 8

4.14 Center disk displacements for the GEL lubricant cases 126

4.15 Dynamic stiffness (a) and damping (b) coefficients for cases 9, 10, and 13-16 127

5.1 A 3D solid model depiction of the GEL Bearing Test Rig test section 133

5.2 Reuland Electric motor to be used with the GEL bearing testbed 134

5.3 Reuland Electric motor specifications. 134

5.4 Rexnord Thomas 150 SR71 double-flex coupling. 135

5.5 GEL bearing test rig rotor 136

5.6 High-pressure bearing housing for the GEL bearing test rig 137

5.7 High-pressure bearing housing cross-section 138

5.8 Finite element model of the bearing housing 139

5.9 Peak stresses in the high-pressure bearing housing. 140

5.10 Peak stresses in the housing bolts 141

5.11 Separation between the housing surfaces at design pressure

5.12 Deformation of the bearing housing at design pressure

5.13 Critical speed map of the GEL bearing test rig. 143

5.14 Undamped mode shapes of the bearing test rig rotor 144

5.15 Damped mode shape of the first critical speed 146

5.16 Unbalance response of the second mode in one of the test rig bearings 147

5.17 The GEL delivery system for the bearing test rig 148 
5.18 Floating bushing seal for the high-pressure bearing housings

6.1 A 3D solid model depiction of the GEL Seal Test Rig test section 156

6.2 The Giordano Colombo spindle motor driving the GEL seal test rig 157

6.3 The GEL Seal Test Rig rotor. 158

6.4 Cross-sectional view of the seal rig test section 159

6.5 High-pressure ports machined into the seal cartridges for data collection 160

6.6 Stiffness and damping coefficients for the plain annular seal at 10,000 rpm. 162

6.7 Campbell diagram of the frequency response of the GEL seal test rig.... 165

6.8 Finite element model of the GEL seal test rig test section 166

6.9 Peak stresses in the test rig bolts 167

6.10 Peak stresses in the central housing 168

6.11 Peak stresses in the seal. 168

6.12 Modal analysis of the test rig base and support structures 169

6.13 The modified GEL supply system for the GEL Seal Test Rig... 


\section{List of Tables}

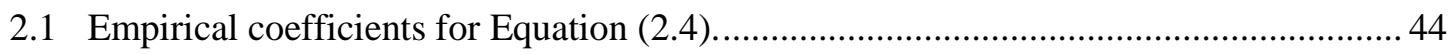

2.2 Diffusion coefficients and Henry's Constants for lubricant- $\mathrm{CO}_{2}$ mixtures.................... 47

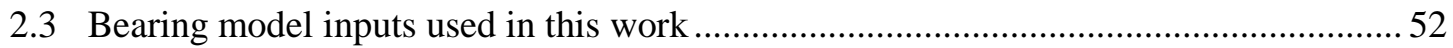

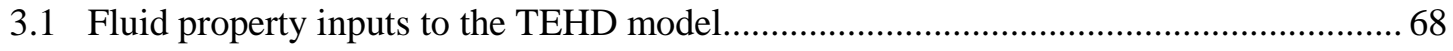

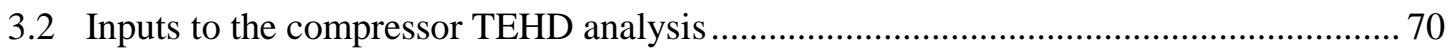

3.3 Inputs to the TEHD analysis for the turbine-generator system .................................... 70

3.4 Critical speeds and log decrements for the turbine-generator system. Both forward (F)

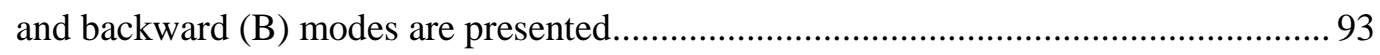

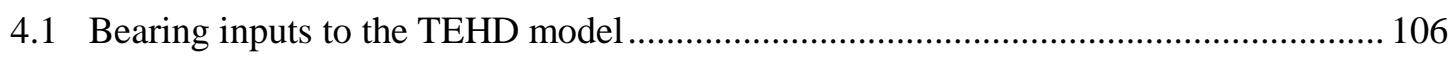

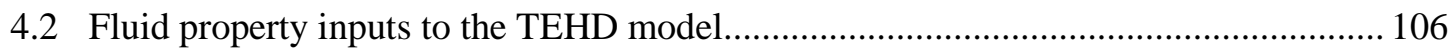

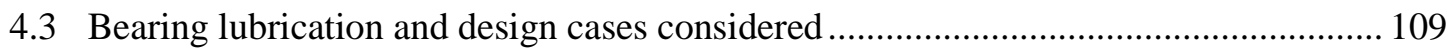




\title{
Nomenclature
}

\author{
$\mathrm{a}_{1}-\mathrm{a}_{5}$ empirical constants \\ $\mathrm{c}_{\mathrm{r}} \quad$ clearance \\ C damping matrix \\ $\mathrm{C}_{\mathrm{P}} \quad$ specific heat \\ d empirical constant \\ D diffusion coefficient \\ $\mathrm{D}_{\mathrm{L}} \quad$ lubricant depth \\ e radial displacement \\ f external force \\ $f_{c} \quad$ coefficient of friction \\ F rub impact force \\ G gyroscopic matrix \\ H Henry’s Constant \\ $\mathrm{k} \quad$ stator stiffness \\ K stiffness matrix \\ L bearing axial length \\ m mass fraction \\ M intertia matrix \\ MW molecular weight \\ $\mathrm{n} \quad$ index
}




\begin{tabular}{|c|c|}
\hline $\mathrm{p}$ & damping exponent \\
\hline $\mathrm{P}$ & pressure \\
\hline$q$ & cross-coupled stiffness \\
\hline Q & cross-coupled stiffness matrix \\
\hline $\mathrm{r}$ & journal radius \\
\hline $\mathrm{R}$ & universal gas constant \\
\hline $\mathrm{t}$ & time \\
\hline $\mathrm{T}$ & temperature \\
\hline $\mathrm{u}$ & displacement vector \\
\hline $\mathrm{v}$ & shaft rotational angular velocity \\
\hline $\mathrm{V}$ & volume \\
\hline $\mathrm{x}$ & circumferential coordinate \\
\hline $\mathrm{X}_{\mathrm{o} 1}$ & horizontal displacement \\
\hline $\mathrm{y}$ & radial coordinate \\
\hline $\mathrm{y}_{\mathrm{o} 1}$ & vertical displacement \\
\hline $\mathrm{z}$ & axial coordinate \\
\hline$\beta$ & scaling factor \\
\hline$\delta$ & $\log$ decrement \\
\hline$\varepsilon_{\mathrm{m}}$ & Eddy viscosity \\
\hline$\theta$ & circumferential angular coordinate \\
\hline$\mu$ & dynamic viscosity \\
\hline$\mu_{\mathrm{e}}$ & effective viscosity \\
\hline
\end{tabular}




$\begin{array}{ll}v & \text { kinematic viscosity } \\ \rho & \text { density } \\ \tau & \text { shear stress } \\ \omega_{\mathrm{d}} & \text { damped natural frequency } \\ \Omega & \text { excitation frequency }\end{array}$

\section{Subscripts}

r radial direction

rx horizontal force direction

ry vertical force direction

$\mathrm{x}$ horizontal displacement at bearing

y vertical displacement at bearing

1,2 fluid mixture component 


\section{Chapter 1}

\section{Introduction}

\subsection{Background}

\subsubsection{Lubrication in Rotating Machinery}

Lubricants play an important role in rotating machinery as they separate solid, rotating surfaces and dissipate heat from these high-friction areas. In bearings, lubricants support the weight applied to the bearing, provide stiffness and damping to the rotating shaft, and protect both the rotating and support components from wear [1-3]. In gear meshes, lubricants provide a thin separating film between gear teeth and reduce the amount of friction taking place [4].

High-speed rotating machines are typically lubricated by two types of oils: mineral oils and synthetic oils. Mineral oils are derived from mined crude oil and are manufactured by fractional distillation and other refining processes such as mixing with organic solvents to remove impurities [5]. Synthetic oils are produced from chemicallymodified low molecular weight hydrocarbons that are carefully polymerized to achieve 
the correct chemical structure and fluid properties. Synthetic lubricants are widely known to have a number of performance benefits over mineral oils including a higher viscosity index, a greater thermo-oxidative stability, and greater resilience under extreme operating conditions. Some synthetic oils are biodegradable as well, making them easier to dispose of. Lubricant additives are also commonly used to enhance the performance of both mineral and synthetic oils and include viscosity index improvers, anti-oxidants, anti-wear additives, corrosion inhibitors, and extreme pressure additives. This dissertation will focus primarily on a new form of lubricant called gas-expanded lubricants (GELs), which are mixtures of synthetic oil and dissolved carbon dioxide. The synthetic oils studied as GELs in this work include pure lubricant base stocks of three commonly used lubricant chemistries in high-speed turbomachinery: polyalkylene glycols (PAG), polyol esters (POE), and polyalpha olefins (PAO). These lubricant classes were selected as they are widely used as high-speed bearing lubricants. In addition to the above mentioned properties, these fluids have also been shown to be highly compatible with solutes such as carbon dioxide [6].

Bearing and gearbox environments in rotating machinery are typically characterized by high surface speeds, requiring a separating lubricant film to reduce wear between these surfaces and dissipate heat resulting from the viscous shear of the fluid. Elastohydrodynamic lubrication theory, governed by the Reynolds Equation, is applied to predict the fluid behavior of these systems [7]. Hydrodynamic lubrication is described as the convergence of a wedge-shaped fluid film that is a result of the motion of the rotating surface. The term "elasto" takes into account the elastic deformation of the solid surfaces being separated by the film. The thermal behavior of the fluid film and the surrounding 
structures are then commonly analyzed using the Energy Equation. Further details of these approaches will be described in subsequent chapters.

These environments are subjected to a wide range of operating conditions, driven primarily by ambient conditions, system design, and operating speed. Operating temperatures typically range from 40 to $100{ }^{\circ} \mathrm{C}$, though many applications exist in more extreme environments as well. Lubricants are typically supplied at pressures of 15 to 40 psi [7], though in the fluid film region local pressures can exceed many hundreds of psi as a result of the applied load to the film. In many applications lubricants can also be delivered at much higher pressures during startup to provide separation between metal surfaces until the fluid film develops as a result of the shaft motion. Hydrostatic bearings, which rely more on hydrostatic fluid pressure than the hydrodynamic action of the fluid to provide support for the rotor, are operated in a similar manner. Dynamic operating conditions are also typical of many applications as a result of fluctuating ambient temperatures, operating speeds, and loading conditions.

The effects of the lubricant and its properties on the performance of bearings are substantial. Because power loss in rotating components is a direct function of fluid shear, which is a function of the lubricant viscosity, efficiency gains can be accomplished by changing out lubricants currently in use with less viscous fluids (Figure 1.1). The viscous shear of the fluid also creates heat, hence lower viscosity fluids also tend to produce lower operating temperatures. However, as the film thickness and load carrying capacity of the fluid also typically decrease with viscosity, careful consideration must be taken in making such operational changes. This has proven to be the case when using very low viscosity fluids such as gases, which are limited in terms of both load capacity and rotor 
damping [8]. Lubricant viscosity also has a significant effect on the forces applied to the rotor by the fluid as it rotates inside the bearing, with lubricant temperature - and its effect on viscosity - affecting the stiffness and damping properties of the fluid film [9]. Lubricant pressure can also affect the stiffness and damping forces applied to the shaft in hydrostatic bearings, with higher pressures often resulting in greater bearing stiffness. In GELs the lubricant viscosity is controlled by the amount of carbon dioxide dissolved into the fluid, which is a function of both pressure and temperature.

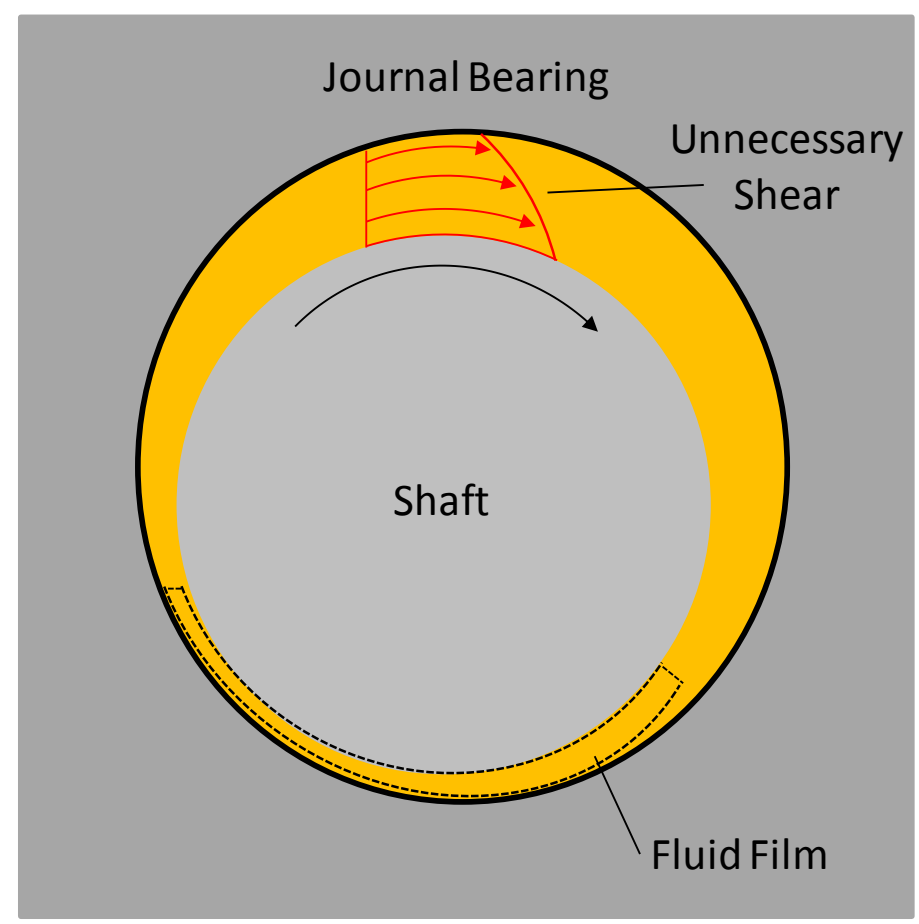

Figure 1.1. Lubricant shear in the non-supporting regions of bearings results in excessive power loss and higher operating temperatures.

Viscosity index (VI), the measure of a fluid's change in viscosity with temperature, has also been shown to affect the energy efficiency of a lubricated system [10]. By having a higher VI, which is typical when comparing synthetics to mineral oils, the fluid viscosity increases less with lowering temperatures, reducing power losses under these 
conditions. Because mineral oils also tend to increase in viscosity as they degrade over time, whereas some synthetic oils do not, synthetics have been shown to generally outperform mineral oils in many performance metrics including energy efficiency $[4,10$ 12]. Other lubricant properties including thermal conductivity and heat capacity have also been found to have an impact on bearing performance, though their effects are smaller when compared to viscosity and viscosity index [10].

As previously described, there are a number of tradeoffs to consider when selecting the proper lubricant for an application. Simply choosing a less viscous fluid to increase efficiency can reduce fluid film thickness and load capacity, resulting in increased wear and premature failure. This leads operators to choose fluids based on worst-case operating conditions which can lead to long-term inefficiencies, particularly in dynamic applications. Conventional mineral oils are also subject to thermo-oxidative breakdown, increasing inefficiencies as they thicken over time as well as leaving sludgy deposits within support components which can also reduce long-term reliability. Pure synthetic fluids offer a higher performing alternative to mineral oils, though they also lack adaptability in dynamic environments, thus also being subject to worst-case design criteria.

Other bearing design and lubricant-based technologies have been studied and utilized in industry to enhance bearing or rotordynamic performance. Directed and leading-edge lubrication techniques, wherein the lubricant is injected directly into the fluid film region, effectively eliminate the need to flood the housing with lubricant [13]. These configurations reduce the amount of fluid shear, and thus, the power loss occurring in the bearing. Other bearing design parameters including bearing clearance, length-to-diameter 
ratio, and the use and design of tilting pads and pressure dams can be used to increase bearing stability [14-18]. Hydrostatic bearings are often employed in smaller, less eccentric machines where the process gas can be used as the lubricant, as well as in larger machines and specialty applications where the hydrostatic pressure can provide lift during low speed operation [7, 19]. However, none of these fluid-based bearings offer any means of real-time bearing and rotordynamic control. Magnetic bearings are utilized in select applications as well, providing the benefits of both greater efficiency and rotordynamic control [20-29], though their use often comes at the expense of high energy consumption, limited load capacity, higher cost, and the requirement for backup bearing systems for energy-loss or control-loss emergency scenarios. Electro-rheological and magneto-rheological fluids have also been proposed as "smart" fluids that could be used to increase bearing performance, though these are still in the early stages of development due to extreme technological demands [30, 31].

\subsubsection{Properties of Lubricant-Carbon Dioxide Mixtures}

As GELs are pressurized mixtures of synthetic oil and carbon dioxide, understanding their properties is essential to understanding their potential effects on bearing performance. Some of the properties of these mixtures have been studied over the past decade by the refrigeration industry as it seeks to identify alternatives to chlorinated refrigerants. Hauk and Weidner [6] studied the potential of using $\mathrm{CO}_{2}$ as a 'green' refrigerant and evaluated a number of these mixtures for their solubility and phase behavior. They found that while most of the mixtures exhibited adequate miscibility at higher temperatures, miscibility limits were demonstrated particularly at low temperatures and with PAO-based mixtures. Hauk [32] also provides a library of 
solubility data for these mixtures in his doctoral thesis, matching experimental data with a Peng-Robinson equation of state. Yokozeki [33] demonstrated that the solubility and phase behavior of these mixtures could be accurately predicted using a Redlich-Kwong equation of state. Youbi-Idrissi and coworkers [34] used an empirical equation to predict the solubility of PAG- $\mathrm{CO}_{2}$ mixtures, also showing good agreement with experimental data. Bobbo and coworkers [35] performed similar solubility analyses with a series of POE lubricants in an effort to determine the most ideal lubricant for refrigeration applications where the refrigerant would be exposed to the oil.

The viscosity of mixtures of synthetic oil and carbon dioxide was reported in the literature by Hauk and Weidner [6] for one each of a PAG, POE, and PAO, though the specific relationship between pressure, the mass fraction of dissolved $\mathrm{CO}_{2}$, and mixture viscosity was only reported for the PAG. This work provided the first evidence that this relationship existed, showing a linear drop in the mixture viscosity with an increasing mass fraction of dissolved $\mathrm{CO}_{2}$ when the viscosity is plotted on a log scale, indicating that this behavior could be controlled via pressure. Hauk's doctoral thesis [32] presents mixture viscosity data for 2 PAGs, 2 PAOs, and 1 POE for a number of isotherms, also showing this behavior. Clarens and coworkers [36] verified this behavior for a lowviscosity PAG and used a modified Arrhenius equation to accurately predict the change in viscosity with increasing mass fraction of $\mathrm{CO}_{2}$ (Figure 1.2). 


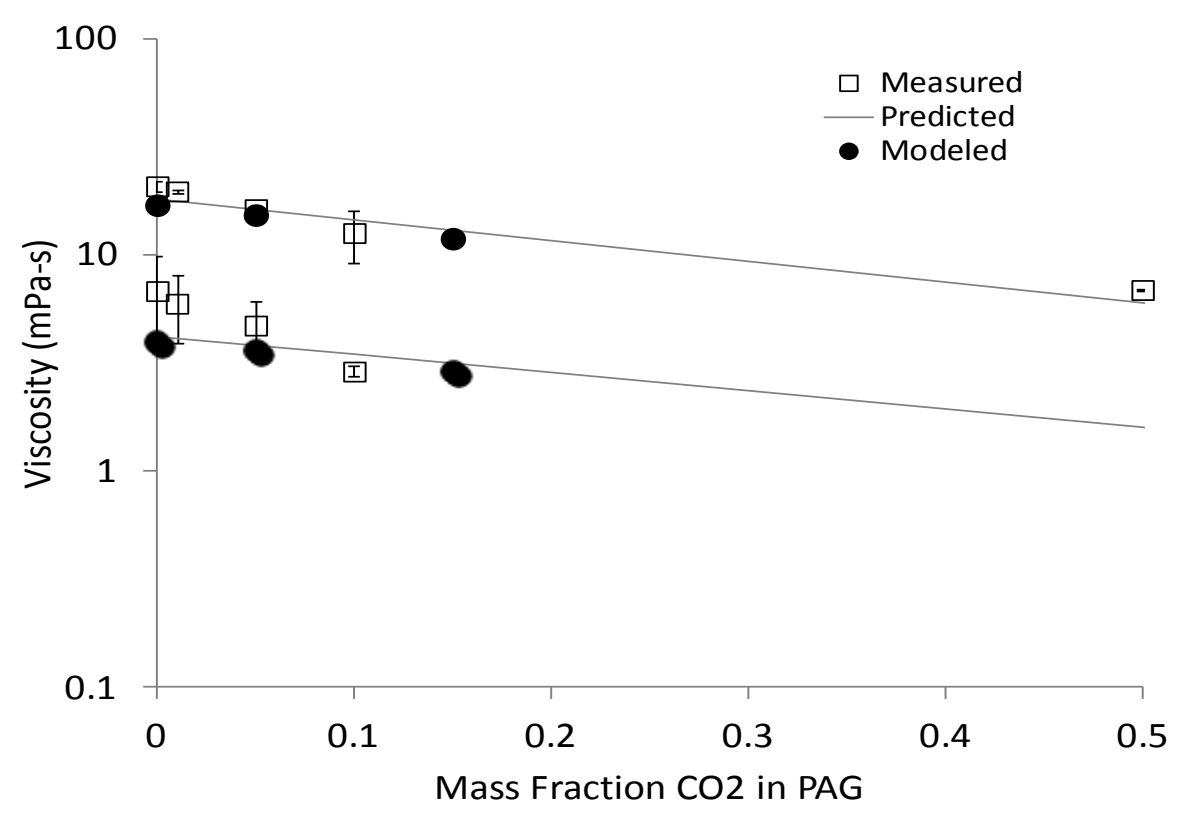

Figure 1.2. Lubricant viscosity at two isotherms as a function of dissolved $\mathrm{CO}_{2}$ mass fraction. From Clarens et al. [36].

The density of synthetic oils has been reported by Hauk [32] to be affected minimally by the addition of carbon dioxide (Figure 1.3). Experimental data was presented for 2 PAGs, 1 POE, and 1 PAO. At low mass fractions the $\mathrm{CO}_{2}$ has little effect due to there being so little solute dissolved into the lubricant. At higher mass fractions, the $\mathrm{CO}_{2}$ density approaches that of the lubricant so the effect at these concentrations is also negligible. 


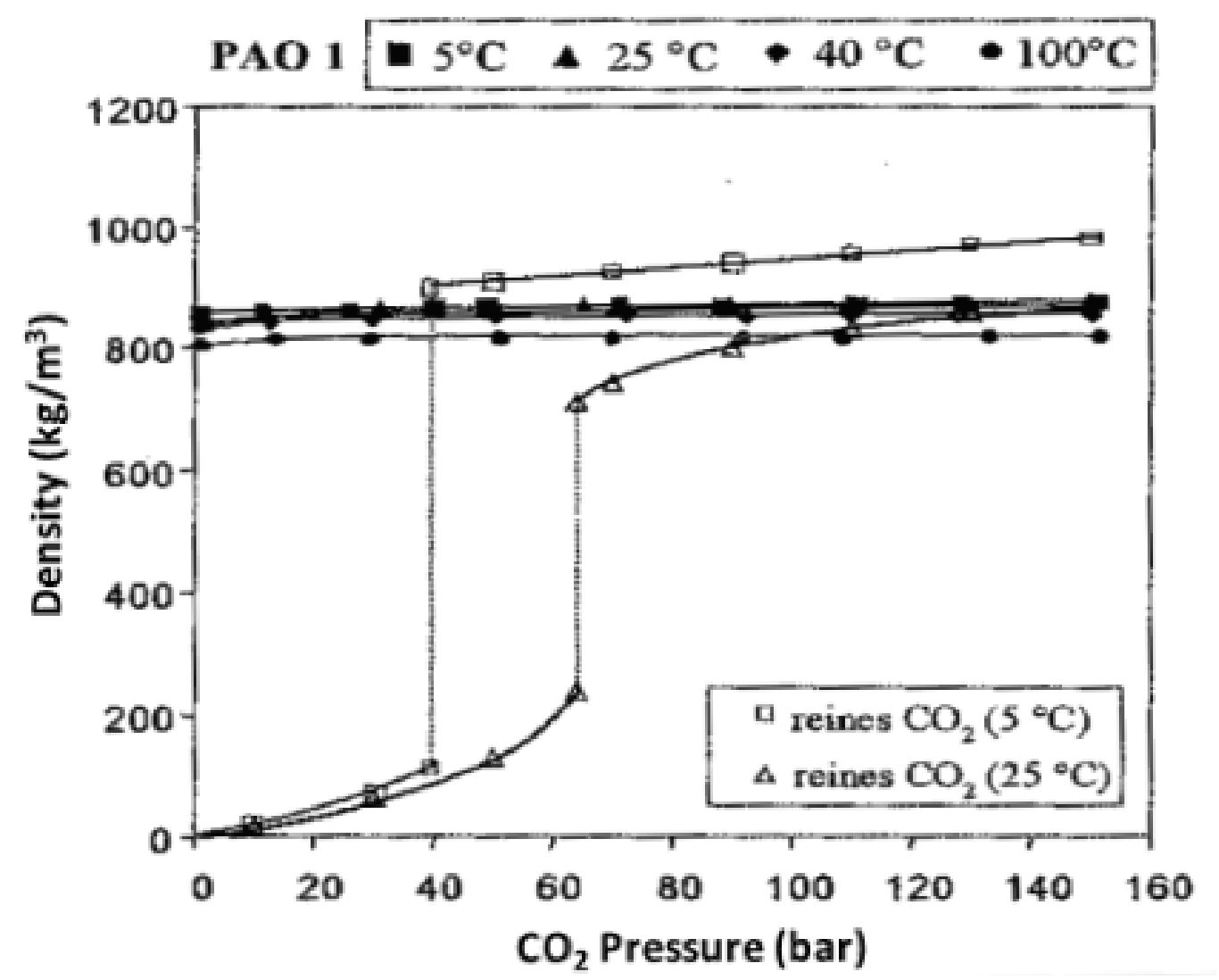

Figure 1.3. Lubricant density remains relatively unchanged for a wide range of pressures and temperatures. Adapted from Hauk [32].

\subsubsection{Effects of Dissolved Solutes on Lubricant Performance}

A number of liquid-liquid and gas-liquid mixtures have been evaluated for their effects on bearing performance in the past. Bair [37] describes how liquid-liquid mixtures can be used to control the viscosity of a mixture, using solutes such as fatty acids, esters, and polymer mixtures. While this may be a promising approach for certain types of applications, it is limited in many applications because of the difficulty inherent in liquidliquid extractions. Some mixtures require extremely high pressures (hundreds of MPa) to lower the viscosity of the fluid. A second drawback is that some polymer mixtures exhibit non-Newtonian, shear-thinning behavior, making it difficult to achieve specified 
fluid properties using this technique. This behavior was modeled by Liu and coworkers [38], with high molecular weight thickeners added to the base lubricant having a minimal effect on film thickness. Thirdly, some of these mixtures are inseparable once they are created, resulting in irreversible control over the fluid properties. Considering oil-water emulsions, Wang and coworkers [39] evaluated their performance using elastohydrodynamic theory. They found that the fluid film thickness varies with oil droplet radius and the inlet volume fraction of oil. It was also found that oil "pooling" typically takes place near the inlet of the lubricated component. This behavior, along with a dependence on speed, often results in these mixtures having inferior lubricating properties as they are typically only used in applications where a high fire-resistance in the fluid is required.

In conventionally lubricated bearings, the fluid film undergoes a pressure spike and subsequent drop as the fluid converges and diverges through the loaded region. In some cases this pressure drop can result in the formation of multi-phase mixtures as well as cavitation, which can result in pitting or other forms of enhanced corrosion. Khonsari and Booser [7] describe two primary forms of cavitation in journal bearings: gaseous cavitation and vapor cavitation. Gaseous cavitation, the most common form, involves phase separation as the fluid film expands in the divergent region of the pad and the pressure drops below the gas saturation pressure. This low-pressure cavity of air is then reintegrated into the fluid as the pressure increases again, resulting in no damaging effects on the bearing. Vapor cavitation, which is more typical in dynamically loaded bearings, results from pressure drops below the vapor pressure of the lubricant, creating air cavities that are then rapidly compressed by the dynamic load. This causes a collapse 
of the air cavity on the surface of the material leading to an erosive damaging effect on the bearing surface.

The Grando group at the University of Leeds has published a number of articles on cavitation and multi-phase flow modeling in journal bearings [40-42]. They reported that mixtures of lubricant with dissolved HFC-134a - which results in a reduction of the mixture viscosity - perform similarly to that of pure lubricant in terms of the pressure distributions across the support region, indicating no detrimental effects in the bearing as a result of the dissolved solute or multi-phase flow. They do, however, point out the need for more information and models on gas absorption as they could only model the system as a single-phase or multi-phase system, not taking into account the actual rate of gas absorption locally. Younan and coworkers [43] modeled the effect of entrained air bubbles in the lubricant in squeeze film dampers and found that the entrained air reduces the load capacity of the fluid while increasing vibration, though it must be noted that the fluid flow in squeeze film dampers is quite different from that in journal bearings.

\subsubsection{Summary}

An overview of conventional lubricants, rotating machinery environments, the effects of lubricant properties on bearing performance has been provided, highlighting the limitations of conventional lubrication technologies. Performance gains have been shown to be achieved by changing lubricants out with fluids having superior properties, modifying the design of the bearing system, or by considering the use of alternative bearing technologies, though many of these techniques are typically limited by design considerations, cost, and a lack of adaptability in dynamic operating conditions. Other fluid mixtures have also been proposed as better performing alternatives to conventional 
fluids, though these are also limited in terms of control and overall performance. The known properties of mixtures of synthetic lubricants and carbon dioxide have been described, though many gaps in the literature still remain on this topic prior to this work, including the thermal properties of these mixtures as well as data on how these mixtures form. Information on the bearing and rotordynamic performance of these mixtures has also previously been unknown, as well as their implications for bearing cavity and seal design. The following sections outline the important need for an alternative, tunable lubricant technology as well as the technology proposed and studied in this work.

\subsection{Problem Statement}

Lubricants are needed for the proper function of most rotating machinery and greatly enhance the efficiency and reliability of a device when deployed correctly [44]. But lubricants also introduce inefficiencies of their own. In many high-speed rotating machines, the shear of the lubricant can create an appreciable loss of power in the system [13]. Some rotating machines are more susceptible to higher power losses and operating temperatures than others. These machines tend to have higher rotating velocities, shaft diameters, and bearing clearances [45]. Collectively, these parameters lead to higher surface speeds and thicker fluid films in parts of the bearing. This will result in significant amounts of lubricant shear and Reynolds numbers that can reach levels indicative of turbulent flow (Figure 1.4) which then causes power loss to increase at an even greater rate with speed $[46,47]$. Consequently, machines with large rotors and high rotational speeds such as steam turbines, gas turbines, generators, and centrifugal compressors are ideal candidates for improving bearing efficiency because they are most 
directly affected by these losses. They also tend to be supported by many (e.g., 8-10) bearings, which multiplies this effect.

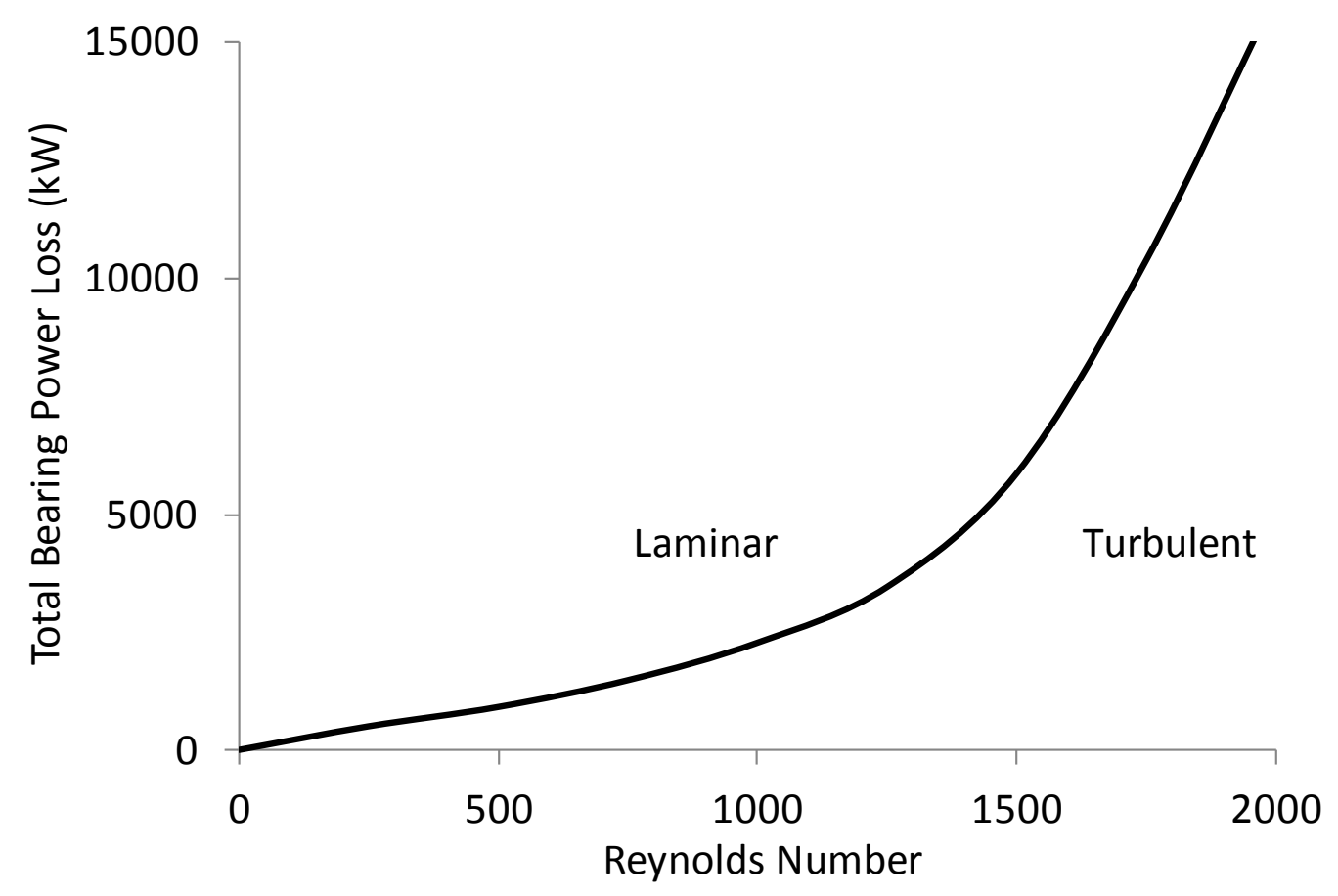

Figure 1.4. Power losses increase drastically as the flow regime transitions from laminar to turbulent flow. Adapted from [47].

\subsection{Technology Concept}

Gas-expanded lubricants (GELs) have been proposed as tunable lubricants with properties that can be adjusted in real time to increase efficiency, reliability, and control in rotating machines (Figure 1.5). GELs are binary mixtures of synthetic lubricant and dissolved carbon dioxide with properties that can be adjusted by changing the relative composition of the mixture [36]. The composition can be adjusted by setting the system pressure or by adjusting the flow rate of the two mixture components, which in turn will specify a unique composition with bulk mechanical and thermal characteristics. Preliminary work had suggested that significant power loss reductions in a tilting-pad 
journal bearing were possible using these mixtures when compared to conventional petroleum-based lubricants in a flooded lubrication bearing. GELs are made using synthetic lubricants because their chemistry is more homogeneous than conventional petroleum and the properties of the mixture can be more easily predicted. This has the added advantage of enhancing the thermal characteristics of the fluid since synthetic lubricants tend to have a higher viscosity index and thermal conductivity. As a result, the pad temperatures in GEL systems are predicted to be lower while several other important process parameters such as lubricant film thickness and eccentricity can be maintained within acceptable ranges. As lubricant properties also directly affect the stiffness and damping forces being applied to the machine rotor, GELs stand to also provide a degree of control over the rotordynamics of high-speed rotating machines, allowing operators to maximize rotor stability during startup and shutdown and minimize excessive vibrations that can lead to catastrophic machine failures.

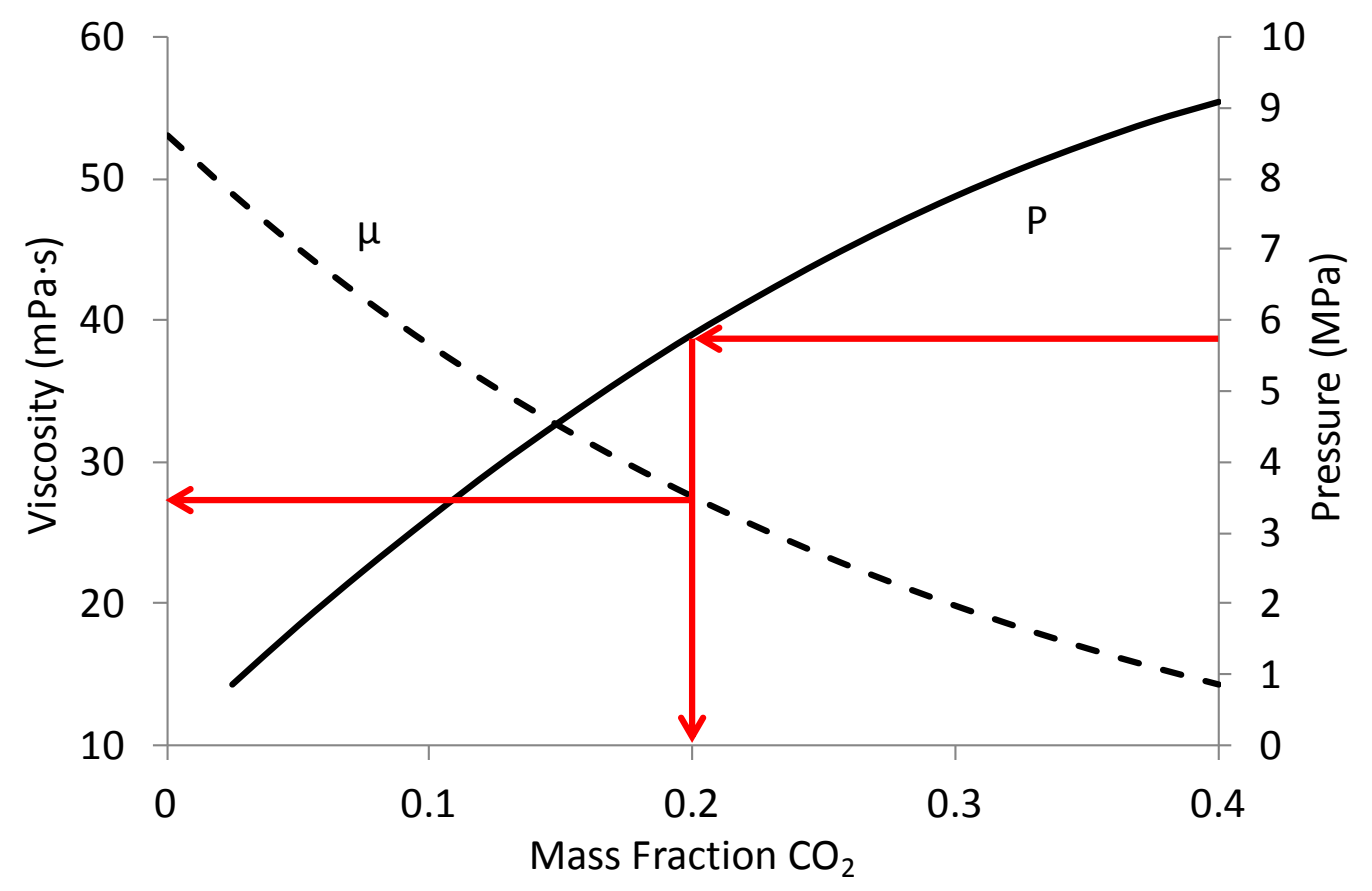

Figure 1.5. Gas-expanded lubricants provide real-time control over lubricant viscosity. 


\subsection{Research Objectives, Scope, and Overview of Dissertation}

The objective of this research is to study gas-expanded lubricants in terms of their properties, performance, and design implications in the context of high-speed rotating machinery. The properties of these fluids will be well-characterized to build upon existing knowledge in the literature. A number of important lubricant properties will be analyzed including mixture viscosity, diffusivity, thermal conductivity, and thermooxidative stability.

This information will then serve as inputs to a number of bearing and rotordynamic performance models for predicting their performance as it compares to that of more commonly used lubricants. Mixture phase behavior will be considered in a tilting pad journal bearing to investigate the potential for bearing cavitation. The influence of laminar vs. turbulent flow in GEL-lubricated bearings will also be considered. Lubricant performance will be evaluated for a wide range of bearing operating speed and loading conditions with performance metrics including power loss, pressure and temperature profiles, minimum film thickness, journal eccentricity, and stiffness and damping coefficients. Lubricant effects on machine rotordynamics will also be analyzed for a number of representative case studies, with critical speeds, mode shapes, mode stability, and rotor displacements considered as performance metrics. The transient control of machine rotordynamics will also be studied along with the use of GELs in mitigating harmful vibration.

Finally, as gas-expanded lubricants require elevated pressures in lubricated components, the design of GEL-compatible systems will be discussed and explored in the context of the design of two GEL test rigs. The first will explore GEL-lubricated bearings 
and the design of high-pressure bearing housings. The design and assembly of a GEL supply system will also be discussed. The second test rig will explore the design and assembly of high-pressure seals for containing these fluids in high-pressure, high-speed rotary environments. Other important considerations including machine operation, system-level efficiency, and machine rotordynamics will also be discussed in the context of these designs as these considerations will be vital to the successful implementation of the GEL technology in modern day machines.

Following this introductory chapter, Chapter 2 will present the results of work performed to characterize the properties of GELs, with bearing performance and fluid phase behavior also being considered. Chapter 3 will extend the analysis of GEL performance to larger, more heavily loaded bearings from real machine designs while also considering the effects of lubricant properties on the rotordynamic performance of the machines considered. The transient control and analysis of machine rotordynamics will also be discussed in this chapter. Chapter 4 will explore the use of GELs in minimizing vibration to improve or remove rub impact conditions in a three-disk rotorbearing system. The design and assembly of a GEL bearing test rig and a GEL seal test rig will be discussed in Chapters 5 and 6, respectively. Finally, Chapter 7 will provide conclusions and recommendations for future work studying GEL performance and further developing the technology for both academic exploration and commercial use. 


\section{Chapter 2}

\section{Properties and Performance of Gas-}

\section{Expanded Lubricants in Tilting Pad Journal}

\section{Bearings}

\subsection{Introduction}

In this work, experimental results of GEL viscosity, gas diffusivity, thermo-oxidative stability, and thermal conductivity were combined with high-pressure phase equilibrium data to understand how these mixtures will behave in tilting-pad journal bearings under a range of industry-relevant high-speed conditions. Simulations were carried out using the experimental data as inputs to a thermoelastohydrodynamic model of tilting-pad journal bearing performance. Phase behavior within the bearing was also simulated using pressure-temperature distributions and an empirical GEL solubility model.

As described in Chapter 1, lubricant cavitation has the potential to produce an erosive, damaging effect in bearings, depending on the type of cavitation occurring. This

*Originally published by STLE. See reference [79]. 
work tests the hypothesis that GELs will not exhibit significant cavitation in journal bearings using a combination of experimental and modeling tools. The results of this analysis help establish the feasibility of using GELs over a range of operating conditions and GEL properties to understand how the technology could be deployed. The relationship between carbon dioxide composition in the GEL and viscosity is reported for a library of representative lubricants and the power loss from using these lubricants in a bearing is reported. The thermodynamic and thermochemical properties of GELs are also studied and incorporated into the modeling effort.

\subsection{Experimental Methods}

\subsubsection{Diffusivity}

The diffusion of carbon dioxide into various fluids [48-60] and the diffusion of refrigerants into lubricant oils [40, 61-64] are described in the literature. Despite this effort, the diffusion of carbon dioxide into synthetic oils had yet to be reported. Hence, $\mathrm{CO}_{2}$ diffusivity into a group of representative lubricants was measured using a modified pressure-decay method developed by Hou and Baltus [57]. Lubricant was added gravimetrically to a pressure vessel of known volume and dimensions. The vessel was sealed, placed into a $25^{\circ} \mathrm{C}$ water bath, and pressurized with $30 \mathrm{psi}$ of $\mathrm{CO}_{2}$. The vessel was then thermally isolated and the pressure was recorded using a pressure transducer (Omega Engineering PX41). The $\mathrm{CO}_{2}$ diffused into the lubricant until an equilibrium pressure was achieved. The time to reach equilibrium ranged between 20 hours to 7 days, depending on the molecular weight of the fluid. Each pressure curve could then be fit by non-linear regression to Equation (2.1), derived from Fick's Law for one-dimensional 
diffusion, Henry's Law, and a mole balance of the gas column above the fluid film. The full derivation of Equation (2.1) can be found in [57].

$$
\begin{gathered}
\ln \frac{P}{P_{0}}=\left(\frac{k}{H_{\mathrm{CO}_{2}}}\right) \sum_{n=1}^{\infty} \frac{1}{(2 n+1)^{2}}\left\{\exp \left[-\frac{(2 n+1)^{2} \pi^{2} D_{\mathrm{CO}_{2}} t}{4 D_{L}^{2}}\right]-1\right\} \\
\text { where } \quad k=\frac{8 R T V_{\text {Oil }} \rho_{\text {Oil }}}{\pi^{2} V_{\mathrm{CO}_{2}} M W_{\text {Oil }}}
\end{gathered}
$$

The regression resulted in outputs of diffusion coefficients, $\mathrm{D}_{\mathrm{AB}}\left(\mathrm{cm}^{2} / \mathrm{s}\right)$, and Henry’s Constants, H (bar). As Henry's Law is only appropriate for predicted solute mole fractions at very low concentrations, this parameter could be used for method verification purposes but is generally less important when considering higher concentration lubricant$\mathrm{CO}_{2}$ mixtures. The diffusion coefficients do provide some insight into how gas-expanded lubricants form, how the two components separate, and what physical mechanisms are the primary drivers of this process.

The accuracy of this method was verified by measuring the Henry's constant for a mixture of carbon dioxide and n-dodecane. Henry's constants for this mixture were published by Henni and coworkers [65], with an empirical equation developed for predicting the values at any temperature. At $25^{\circ} \mathrm{C}$ this value was calculated to be 79.36 bar, within $3.3 \%$ of our experimentally measured value of 76.72 bar. Two replicates were measured for each lubricant, with the cutoff time for the data used in the regression established by the shorter of the two replicates.

\subsubsection{Viscosity}

The viscosity of the pure lubricants and GELs was measured using an Anton Paar MCR 301 rheometer equipped with a high-pressure cell rated to $15 \mathrm{MPa}$. The $\mathrm{CO}_{2}$ was 
delivered to the pressure cell using a Teledyne ISCO 500HP syringe pump with a constant-temperature jacket. The temperature jacket ensured the delivery of liquid $\mathrm{CO}_{2}$, allowing for the calculation of the $\mathrm{CO}_{2}$ mass fraction via volumetric measurements and mass balance calculations. The temperature of each sample was controlled to within $\pm 0.1^{\circ} \mathrm{C}$ using a Peltier style temperature controller integrated into the rheometer. All measurements were performed at $40^{\circ} \mathrm{C}$ and $100^{\circ} \mathrm{C}$ which represent a typical operating temperature range in bearing and gearbox environments.

\subsubsection{Thermo-oxidative Stability}

The thermo-oxidative stability of the pure lubricants and GELs was also evaluated, using viscosity as an indicator of degradation, by monitoring the viscosity of the lubricants subjected to high temperatures and pressures over six weeks. The synthetic lubricant base stocks tested contained no antioxidants and were susceptible to thermooxidative breakdown under the conditions tested here. The petroleum oil benchmark used in this study did contain phenolic antioxidants but since this is an industry standard for these applications, it was appropriate to benchmark its performance to the synthetic fluids selected. Our analysis represents a conservative analysis of the potential of synthetic lubricants in GELs. The use of viscosity as an indicator of oxidative stability has been used in the past by other researchers to analyze the oxidative stability of various fluids, including lubricants, often in conjunction with one or more other metrics of oxidation such as Total Acid Number (TAN) or Oxidation Induction Time (OID) [66-69]. Methods that utilize these metrics, including Differential Scanning Calorimetry (DSC) [66-68, 70], Total Acid Number [66, 69, 70], and the Rotary Bomb Oxidation Test (RBOT) [68, 70] were also considered but were found to be challenging to apply to a pressurized $\mathrm{CO}_{2}$ 
environment since these methods typically require the use of pressurized oxygen atmospheres (RBOT), oxygen flows (DSC), or reagents that could react with the carbon dioxide (TAN). Two replicates each of pure lubricant and GEL pressurized to 2.76 MPa were loaded into sealed pressure vessels and placed into an oven at $100^{\circ} \mathrm{C}$. Each week the samples were cooled to room temperature and the $\mathrm{CO}_{2}$ was removed from the GELs by depressurization and a vacuum pump. Samples were then measured for viscosity at $40^{\circ} \mathrm{C}$ before being pressurized and placed back into the oven.

\subsubsection{Thermal Conductivity}

The thermal conductivity of three representative GEL chemistries was measured using a Decagon KD2 Pro equipped with a KS-1 sensor for measuring the thermal conductivity of liquids. All measurements were performed at room temperature in a thermally stable laboratory to minimize any error in the measurement associated with convection.

\subsubsection{Test Lubricants}

Seven lubricant base stocks representing three of the most common synthetic lubricant chemistry classes were analyzed to calculate a $\mathrm{CO}_{2}$ diffusion coefficient and Henry's Constant. The lubricants included here are: three polyalkylene glycols (PAGs) (Dow Chemical Company, Midland, MI), three polyalpha olefins (PAOs) (Chemtura and ExxonMobil Chemical, Fords, NJ and Houston, TX), and one polyol ester (POE) (Chemtura, Fords, NJ). These three synthetic oil types are widely used in bearing and gearbox applications. The lubricant selection within each class was based on viscosity grade and molecular structure. A total of eleven lubricants were evaluated for viscosity, diffusivity, thermal conductivity, and thermal stability as pure fluids and as GELs. 
The lubricant used for modeling GEL phase behavior in a tilting pad journal bearing was a POE because POEs are known to be highly compatible with $\mathrm{CO}_{2}$ and because extensive literature data is available detailing the high pressure fluid phase behavior of $\mathrm{POE} / \mathrm{CO}_{2}$ mixtures. A combination of experimental and published parameters was used as inputs to the model. Viscosity and carbon dioxide solubility data were obtained from Hauk [32] for a POE 3. The effect of dissolved carbon dioxide on the viscosity of the GEL was measured experimentally and the results were fit using a modified Grunberg and Nissan Equation, which has been used before to accurately predict the viscosity of these mixtures [71].

$$
\ln \mu_{M i x}=m_{1} \ln \mu_{1}+m_{2} \ln \mu_{2}+m_{1} m_{2} d
$$

The density was obtained from the manufacturer of a POE 2926 lubricant. Hauk [32] had previously reported that the effect of dissolved carbon dioxide on mixture density was minor. The properties of the POE 3 and the POE 2926 were considered together and found to be consistent since both had similar chemistry and viscosities. The specific heat of the pure lubricant was estimated from values reported by Totten and coworkers [72] for a polyol ester. Again here, the effects of temperature and pressure on pure lubricant thermal properties were assumed to be negligible for the ranges considered in this study as demonstrated in [73]. The Jensen Equation [74] was used to calculate the specific heat of the lubricant-carbon dioxide mixture.

$$
C_{P_{M i x}}=m_{C_{2}} C_{P_{\mathrm{CO}_{2}}}+\left(1-m_{\mathrm{CO}_{2}}\right) C_{P_{\mathrm{O} i l}}
$$




\subsection{Modeling Framework}

\subsubsection{Solubility of Carbon Dioxide in Lubricant}

The solubility of carbon dioxide in POE was calculated as a function of temperature and pressure conditions using a set of empirical isotherms developed from experimental phase behavior collected by Hauk [32]. This approach for modeling high pressure phase behavior, shown in Equation (2.4), was used by Jeng and coworkers [75] and YoubiIdrissi and coworkers [34] to describe the behavior of high pressure and temperature mixtures of synthetic oil and refrigerants including carbon dioxide.

$$
P=a_{1} m+a_{2} m T+a_{3} m^{2}+a_{4} m^{2} T+a_{5} m^{2} T^{2}
$$

The fit was performed using a nonlinear regression in the MATLAB Surface Fitting Toolbox. The empirical coefficients calculated by the regression are given in Table 2.1. Figure 2.1 illustrates that the solubility model matches the experimental data well for the temperatures and mass fractions of carbon dioxide expected in a GEL-lubricated bearing. The average relative error between the experimental data and their fit is $1.2 \%$. GELs do not easily form at $\mathrm{CO}_{2}$ mass fractions much higher than $20 \%$ because the pressure required to maintain an equilibrium mixture at that composition is very high, especially at higher temperatures. Since the model only needed to cover the mass fraction range from $0-20 \%$, this approach was more computationally efficient than using an equation of state while maintaining comparable accuracy. These phase behavior data were then used to specify other bearing model inputs including supply pressure, GEL viscosity, and specific heat. 
Table 2.1. Empirical coefficients for Equation (2.4).

\begin{tabular}{ccccc}
\hline \hline$a_{1}$ & $a_{2}$ & $a_{3}$ & $a_{4}$ & $a_{5}$ \\
\hline-150.3 & 0.5926 & 525.1 & -3.317 & 0.004917 \\
\hline \hline
\end{tabular}

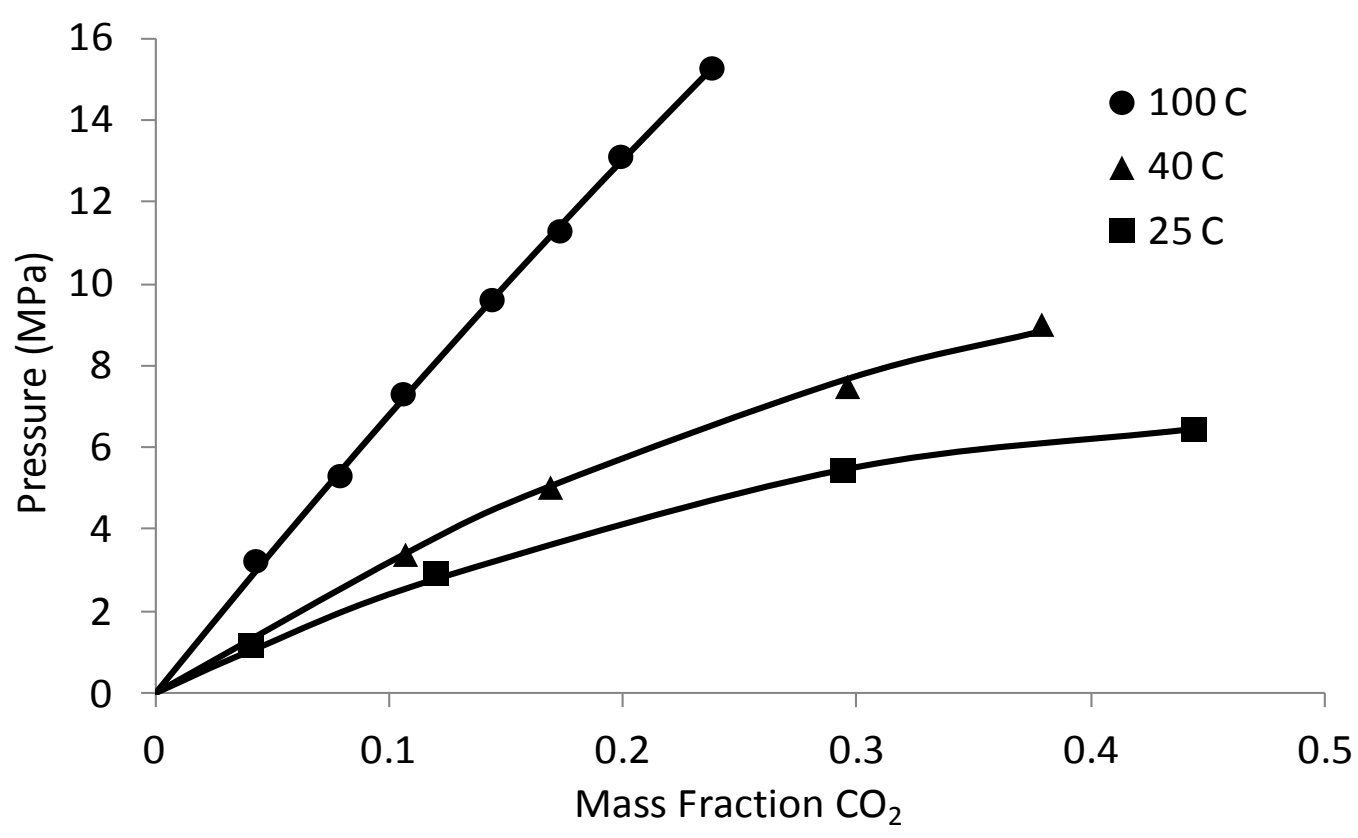

Figure 2.1. High-pressure fluid-phase stability curves for POE and carbon dioxide. GELs form above the isotherms; below them multiple phases exist. Experimental data are from Hauk [32] and model results are from this work.

\subsubsection{Thermoelastohydrodynamic Bearing Model}

The bearing model employed in this study is a well-established TEHD analysis [7678] that has also been previously used to model GEL performance in tilting pad journal bearings [31, 36, 79]. The model is based on the two-dimensional forms of the generalized Reynolds and energy equations, which enable the study of lubricant viscosity and its effects on operating temperature, power loss, eccentricity, and stiffness and damping behavior. The effectiveness of this model has been demonstrated and paired 
with experimental data from Taniguchi and coworkers [80]. Key assumptions made with this approach include a constant pressure profile across the film thickness, no slip conditions at surfaces, negligible inertia effects, constant lubricant densities, and fully flooded bearings with $80 \%$ hot oil carry over between pads. Instantaneous mixing of the lubricant locally is also assumed. This assumption is reasonable due to the large amount of shear taking place in the bearing. Lastly, the thermal expansion term in the energy equation is assumed to be negligible. Due to the small change in pressure per node, the resulting compressive heating and cooling term is found to be multiple orders of magnitude smaller than the conduction and convection terms. The generalized Reynolds equation allows for the variation of the viscosity across the film as well as for the presence of turbulence. The effect of turbulent stress is combined with the eddy viscosity law $\left(\varepsilon_{\mathrm{m}}\right)$ to express the viscosity as an effective viscosity $\left(\mu_{\mathrm{e}}\right)$ in the Reynolds equation $[81,82]$.

$$
\mu_{\mathrm{e}}(\mathrm{x}, \mathrm{y}, \mathrm{z})=\mu\left(1+\beta \frac{\varepsilon_{\mathrm{m}}}{v}\right)
$$

Local Reynolds numbers are used to determine the flow regime around the bearing, with values below 500 and above 1000, respectively, being considered for laminar and turbulent flow. A scaling factor $(\beta)$ modifies the eddy viscosity for the transitional region between these values. Power loss is calculated using Petroff's equation for concentric cylinders [83], though the concentric assumption is dropped to take journal eccentricity into account. Petroff's equation highlights the direct relationship between power loss and fluid shear, which is a direct function of the fluid viscosity.

$$
\mathrm{P}_{\text {loss }}=\mathrm{rv} \int_{0}^{\mathrm{L}} \int_{0}^{2 \pi} \tau(\theta, \mathrm{z}) \mathrm{d} \theta \mathrm{dz}
$$


This analysis produced full stiffness and damping coefficients for the bearings that are whirl-frequency independent but that consider small perturbations of the shaft position by solving the perturbed Reynolds equation. The bearing analysis code may also synchronously reduce the full coefficients to each shaft rotational speed which produces sets of 8 bearing coefficients for each speed. This allowed the model to be used to simulate the speed-dependent characteristics of the fluid film bearings and how they interact with the entire rotor structure.

\subsection{Results and Discussion}

\subsubsection{Gas-Expanded Lubricant Properties}

The diffusion of $\mathrm{CO}_{2}$ into seven representative lubricants was measured and the results are presented in Figure 2.2. Gas diffusion into lubricants has been shown to correlate well with molecular weight, which is typically proportional to the viscosity in synthetic lubricants. Diffusion has been shown in the past to be a function of solvent viscosity for a variety of fluids including ionic liquids, organic solvents, aqueous solutions, and oils $[60,84]$. This relationship holds for the GELs evaluated here with a $\log$-linear relationship between viscosity and $\mathrm{CO}_{2}$ diffusivity observed. The slope of the relationship between viscosity and diffusivity $(-0.535)$ is highly consistent with values reported by Moganty and Baltus [60] for other fluids (-0.66 to -0.44). For the lubricants measured here, the diffusion coefficients decrease by an order of magnitude between the lowest and highest viscosity lubricants. From a practical standpoint, these data are important in the design of GEL systems because they will drive the design of gas-liquid exchangers and determine the residence times needed to cycle the lubricants through and 
either add or remove gas, depending on the stage in the cycle. As diffusion and lowconcentration solubility information is also relevant for the refrigeration industry, Table 2.2 presents both the diffusion coefficients and Henry's Constants for all of the lubricants analyzed.

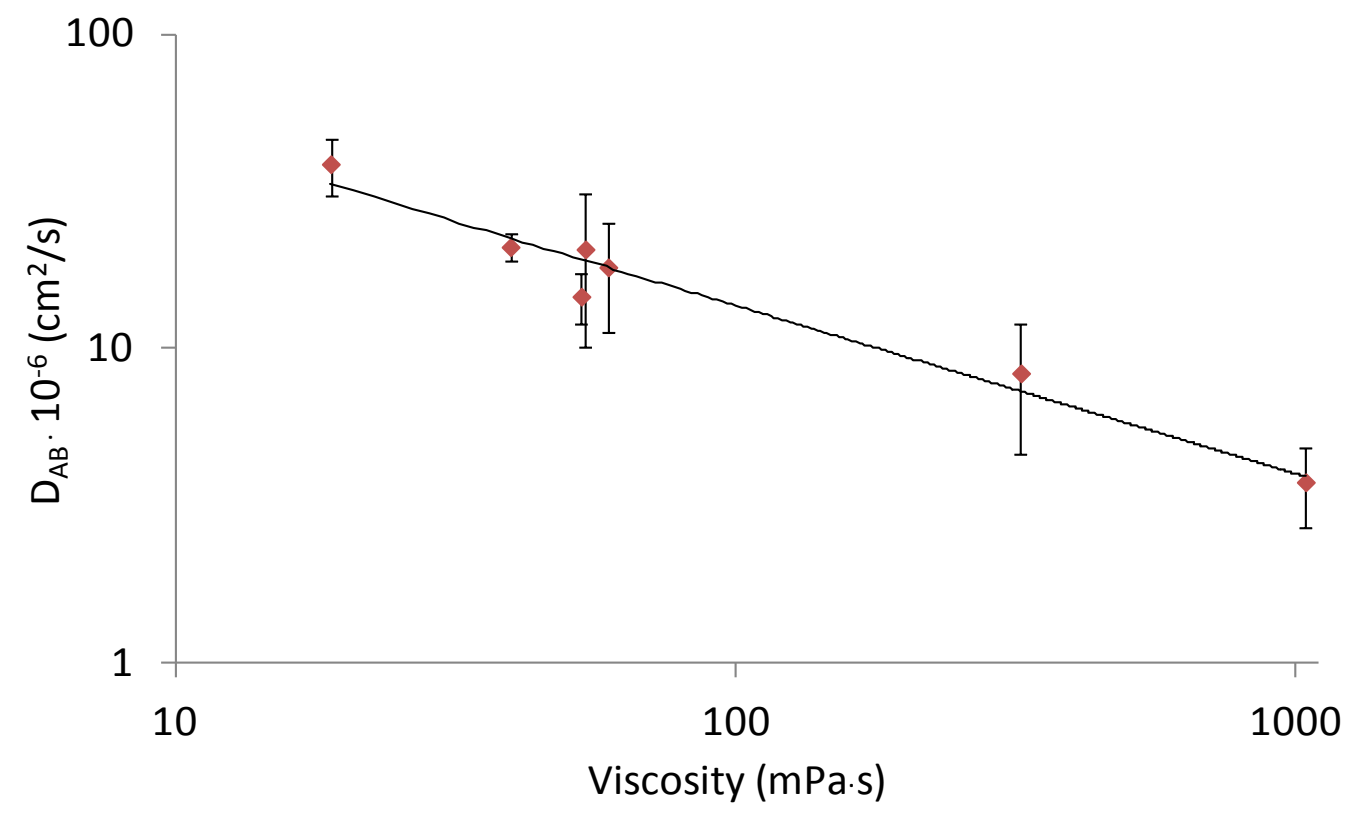

Figure 2.2. Diffusion coefficients as a function of lubricant viscosity. The fitted line has a slope of -0.535 , a relationship found comparable to others reported [60].

Table 2.2. Diffusion coefficients and Henry's Constants for lubricant- $\mathrm{CO}_{2}$ mixtures.

\begin{tabular}{cccc}
\hline \hline Lubricant & $\mu_{40}(\mathrm{mPa} \cdot \mathrm{s})$ & $\mathrm{D}_{\mathrm{AB}}\left(\mathrm{cm}^{2} / \mathrm{s}\right)$ & $\mathrm{H}(\mathrm{MPa})$ \\
\hline PAG 1 & 18.9 & $3.87 \mathrm{E}-05$ & 2.053 \\
PAG 2 & 53.9 & $2.07 \mathrm{E}-05$ & 1.103 \\
PAG 3 & 59.2 & $1.82 \mathrm{E}-05$ & 1.165 \\
PAO 1 & 39.6 & $2.11 \mathrm{E}-05$ & 3.188 \\
PAO 2 & 323.6 & $8.35 \mathrm{E}-06$ & 0.839 \\
PAO 3 & 1044.2 & $3.76 \mathrm{E}-06$ & 0.377 \\
POE 1 & 53.0 & $1.47 \mathrm{E}-05$ & 1.657 \\
\hline \hline
\end{tabular}


The viscosity-mass fraction relationship of GELs for three representative lubricants is shown in Figure 2.3 for compositions up to $20 \% \mathrm{CO}_{2}$. The experimental data was fit using the modified Grunberg and Nissan equation. As discussed by Clarens and coworkers [36], these fluids also exhibit Newtonian behavior for a wide range of shear rates, thus validating the Newtonian fluid behavior assumption in the TEHD model used in this work. These results suggest that the viscosity properties of GELs can be specified using pressure for a range of lubricant chemistries. These data would be used in a controller designed to specify the GEL properties in response to some external signal. These signals would include parameters such as operating temperature and loading, depending on the application. The controller would then modify the $\mathrm{CO}_{2}$ flow rate in the lubricant stream and the pressure inside of the lubricated component to change the viscosity of the fluid to a more optimal value. This change (and subsequent thermodynamic equilibrium) in the system would be achieved relatively quickly compared to experimental diffusion times as the high shear rates encountered in these components would allow for rapid mixing of the lubricant and carbon dioxide. 

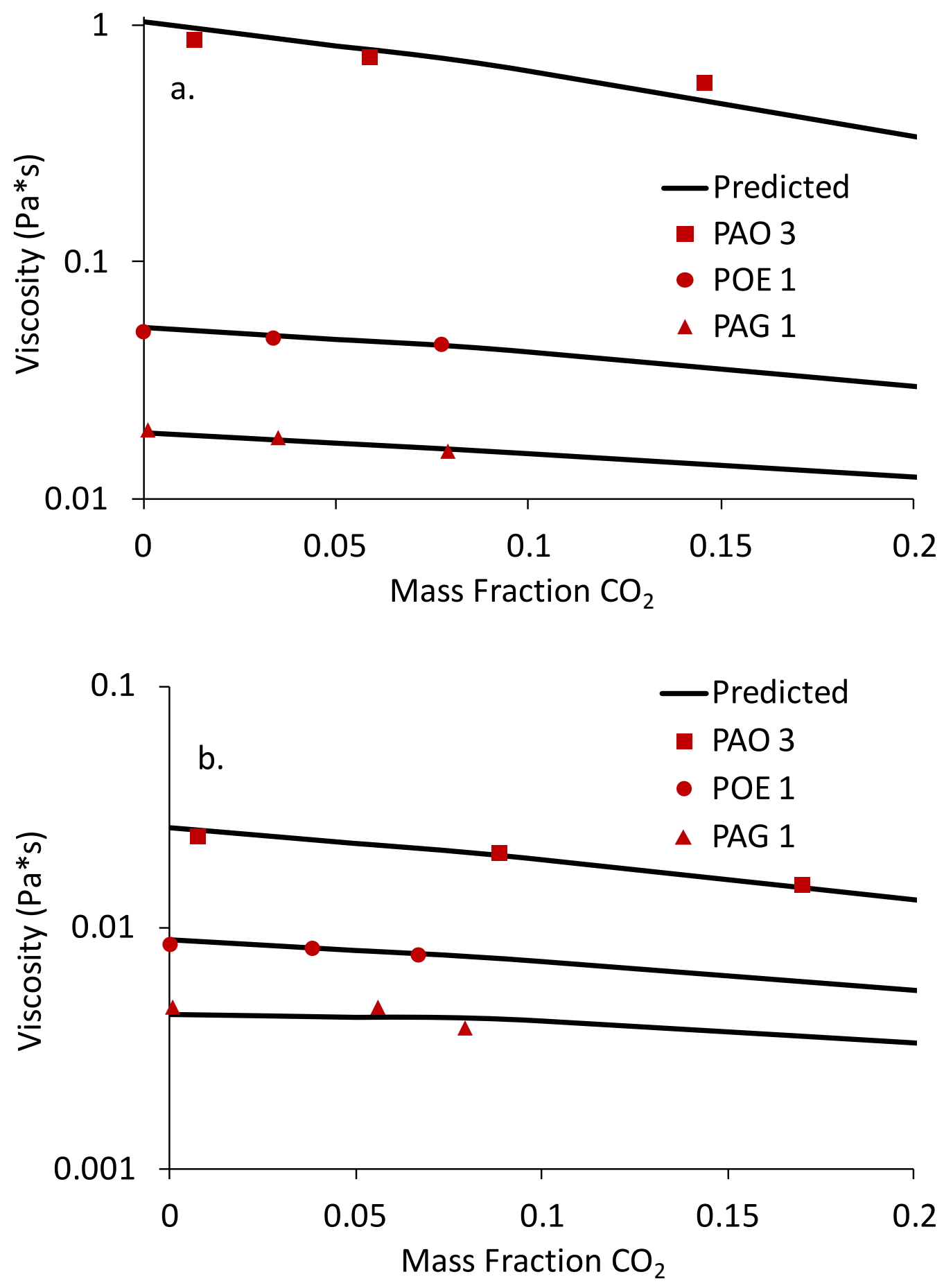

Figure 2.3. GEL viscosity as a function of mixture composition at (a) $40^{\circ} \mathrm{C}$ and (b) $100^{\circ} \mathrm{C}$. 
The thermo-oxidative stability of pure lubricants and GELs, shown in Figure 2.4, was found to be unchanged by the addition of carbon dioxide to the lubricant. In all cases only mild fluctuations in viscosity were found, typically within the margin of error. Visually, all of the synthetic lubricant and synthetic-based GELs remained clear at the end of the six-week period. The petroleum-based ISO VG 68, however, became black as the lubricant began forming higher molecular weight species despite the fact that it contained phenolic antioxidants. This behavior is typical of petroleum-based lubricants as they degrade more quickly than synthetic lubricants. Future studies might explore the impact of higher temperatures or longer contact times as well as other mechanistic connections using modified DSC or TAN methods that are compatible with pressurized carbon dioxide environments.

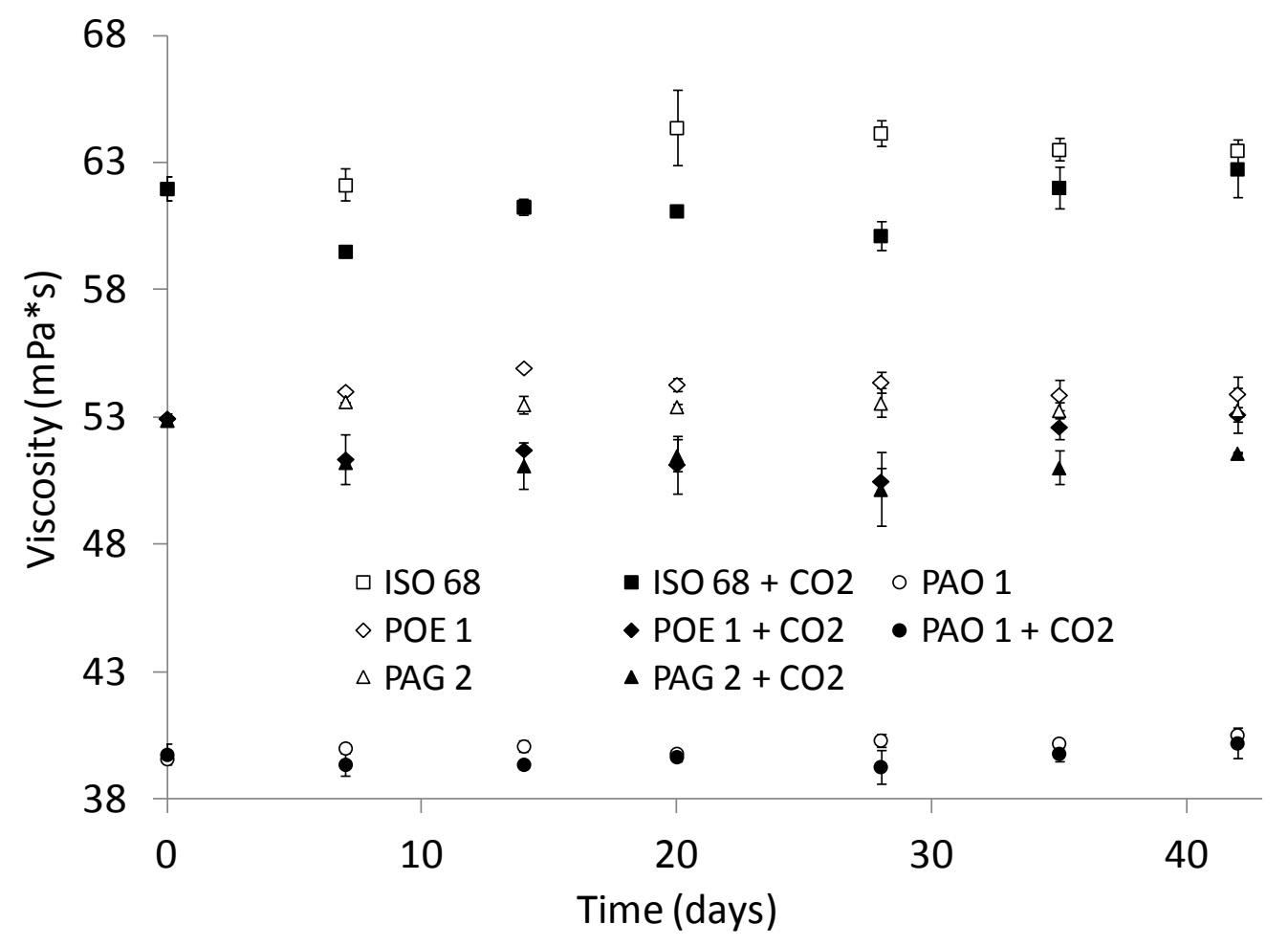

Figure 2.4. Thermo-oxidation of lubricant- $\mathrm{CO}_{2}$ mixtures does not appear to influence the long-term stability of the lubricants compared to lubricants alone. 


\subsubsection{Bearing Performance}

The performance of pure POE and a GEL containing POE and 20\% mass fraction $\mathrm{CO}_{2}$ was modeled using the TEHD finite element code [76] for a range of speeds from 200 to 20,000 rpm. Model inputs are summarized in Table 2.3. Pressure and temperature distributions are shown in Figures 2.5 and 2.6 for the loaded Pad \#4 of the bearing for low- and high-speed cases, respectively. For the low-speed case, both lubricants show little change in temperature as the fluid passes through the loaded region. As expected, the pressure increases by about $0.5 \mathrm{MPa}$ as the fluid passes through the region of high loading and then drops rapidly as the fluid expands and exits this support region beneath the shaft, returning to the supply pressure. This pressure spike is enhanced at higher speeds, shown in the lower panels, with an overall change in pressure of over $7 \mathrm{MPa}$ with the pure POE and $5 \mathrm{MPa}$ with the GEL. Both lubricants exhibit higher operating temperatures under high-speed conditions, with the temperature increasing by approximately $20{ }^{\circ} \mathrm{C}$ across the length of the pad. In this case, the GEL produces lower operating temperatures than the pure POE by approximately 6 to $10{ }^{\circ} \mathrm{C}$. This has important implications for long-term bearing performance as lower operating temperatures are commonly associated with lower rates of wear and increased component longevity. 
Table 2.3. Bearing model inputs used in this work.

\begin{tabular}{lcc}
\hline \hline Parameter & Value & Unit \\
\hline Bearing diameter & 3.81 & $\mathrm{~cm}$ \\
Pad thickness & 1.52 & $\mathrm{~cm}$ \\
Length & 2.54 & $\mathrm{~cm}$ \\
Clearance & 0.03 & $\mathrm{~mm}$ \\
Bearing Preload & 0.5 & \\
Pivot Offset & 0.5 & \\
Configuration & Load On Pad \\
No. of pads & 5 & \\
Oil supply temperature & 40 & \\
Oil supply rate & 1 & $\mathrm{gpm}$ \\
Radial load & 111.2 & $\mathrm{~N}$ \\
Pad thermal conductivity & 50.09 & $\mathrm{~W} / \mathrm{m} \mathrm{K}^{\circ}$ \\
Convection coefficient & 73.6 & $\mathrm{~W} / \mathrm{m}^{2} \mathrm{~K}$ \\
\hline \hline
\end{tabular}



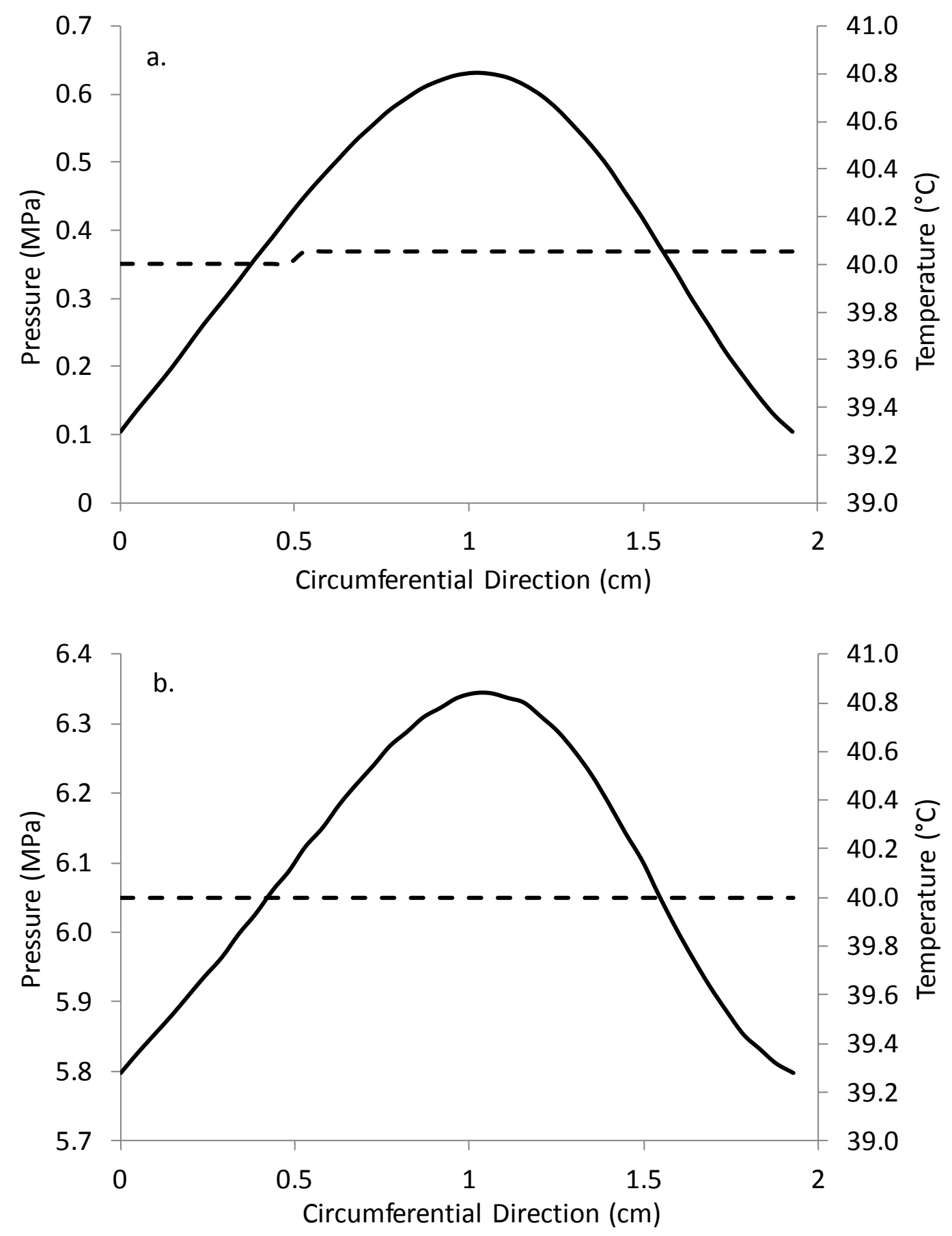

Figure 2.5. Pressure (solid lines) and temperature (dashed lines) distributions on the loaded pad \#4 of the tilting pad journal bearing. Profiles are shown for (a) POE and (b) the POE-based GEL at $200 \mathrm{rpm}$. 

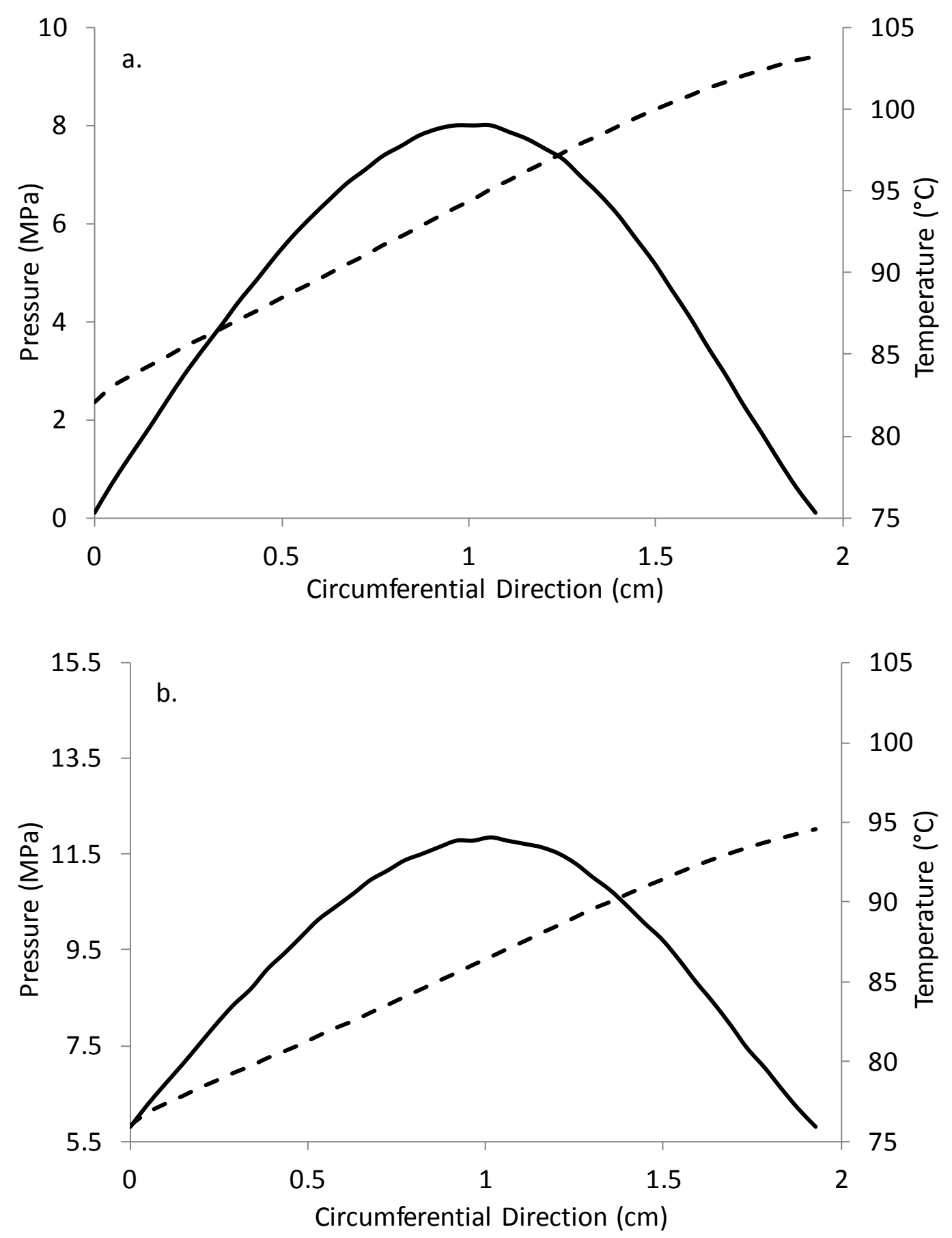

Figure 2.6. Pressure (solid lines) and temperature (dashed lines) distributions on the loaded pad \#4 of the tilting pad journal bearing. Profiles are shown for (a) POE and (b) the POE-based GEL at 20,000 rpm. 
To evaluate the likelihood that a bearing operated using GELs would not produce a multi-phase fluid film, the pressure and temperature distributions from the analysis above were substituted into the Jensen solubility model to determine the composition of the GEL as a function of position in the fluid film. This approach assumes a uniform distribution of carbon dioxide in the bearing due to mixing, as well as thermodynamic equilibrium in the form of instantaneous mixing at all locations within the bearing. Figure 2.7 illustrates how the solubility of carbon dioxide in the GEL changes as a function of position for the low and high-speed cases. For the low speed case, the pressure spikes but never falls below the supply pressure at constant temperature, therefore the $\mathrm{CO}_{2}$ solubility remains constant through the region of support because mass transfer is limiting carbon dioxide dissolution into the lubricant.

The high-speed case presents a more relevant discussion on the solubility of the GEL and the operational considerations involved in the local phase behavior. High-speed systems are more likely to adopt GELs because of the greater overall energy savings. As with the low speed case, an increase in pressure is observed through the loaded region on Pad \#4. There is considerably more heat in this system and the operating temperatures across the pad begin at values above the supply temperature and increase across the length of the pad. $\mathrm{CO}_{2}$ solubility increases with the pressure build up on the pad but because of the higher temperature the overall value is low and it drops to its lowest value at the end of the pad. 

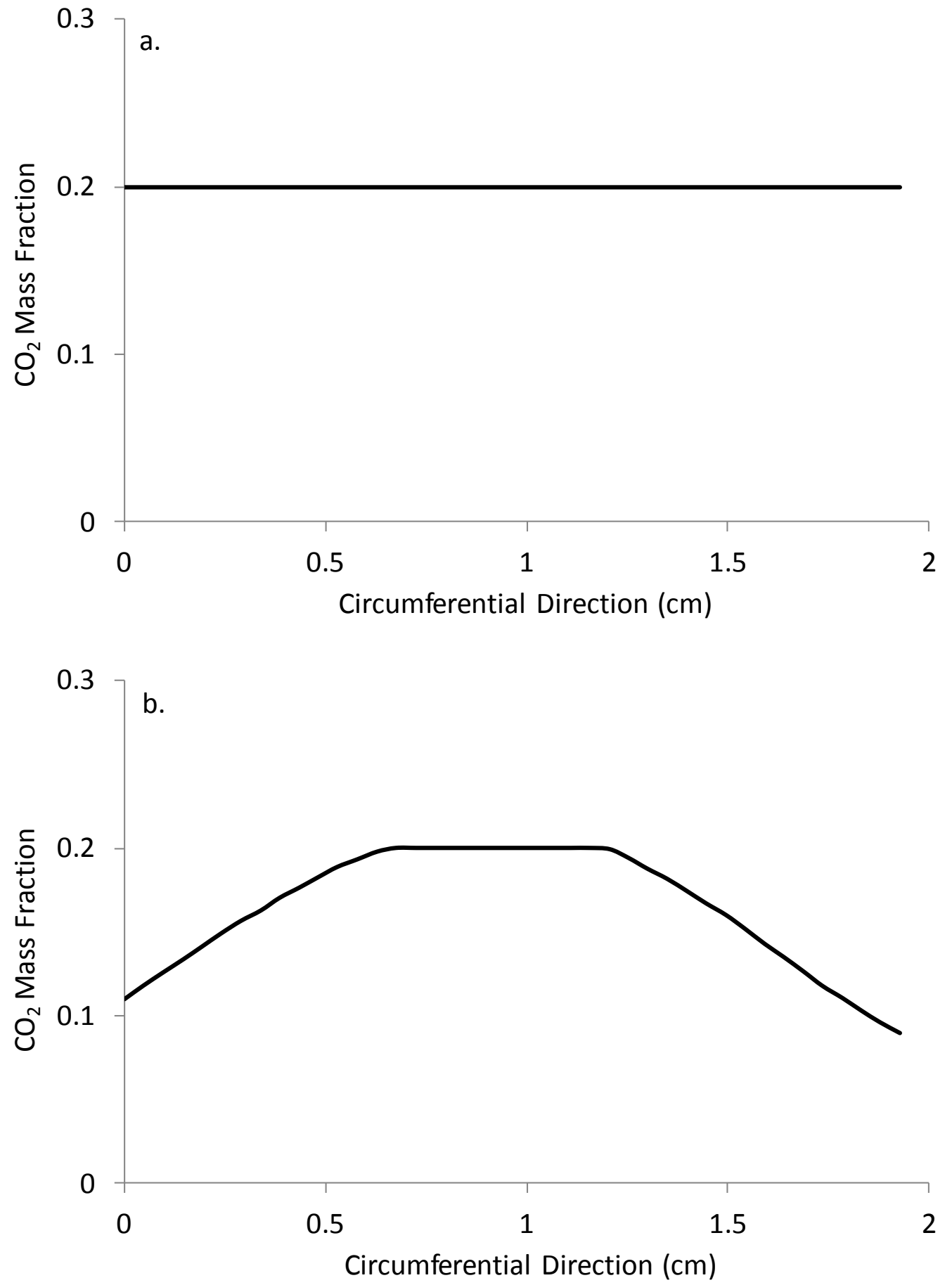

Figure 2.7. GEL composition in the fluid film region along pad \#4 of the tilting pad journal bearing at (a) $200 \mathrm{rpm}$ and (b) 20,000 rpm. 
In a bearing operated as in the low speed case, these conditions could result in multiphase flow through the region of support because not all of the carbon dioxide would be dissolved into the lubricant locally. This behavior is commonly avoided by adjusting the $\mathrm{CO}_{2}$ flow rate or by modifying the operating pressure. This would effectively control the mixture such that the amount of carbon dioxide delivered to the bearing would only be enough to reach the solubility point of the minimum pressure and maximum temperature conditions, eliminating the solubility spike and subsequent drop as the fluid exits the region of support. Since changes to these operating inputs would have a direct effect on the temperature of the bearing, any adjustments to the system would be performed in a stepwise fashion to enhance the control over the mixture properties. Maintaining proper film thickness would remain an important consideration in these operational decisions as well.

In an effort to avoid localized phase separation and cavitation, additional simulations were conducted to understand the operating conditions that could produce pressure drops below the ambient supply pressure. A second low speed case was performed using twice the previously used radial clearance in the bearing. For both the pure POE and GEL, the pressure and temperature distributions appeared similar to those in Figure 2.5, with the only exception being a slight drop $(<0.1 \mathrm{MPa})$ below the ambient pressure in the divergent region of the fluid film. These results indicate that even though localized phase separation is possible under certain sets of operating conditions, this separation does not occur in the critical region of load support and is no more likely to be detrimental to the bearing than a standard lubrication environment. 
These results suggest that cavitation in GELs would not be any more acute than in conventionally lubricated bearings, but the effects of this phase behavior on power loss were also of interest. The power loss estimates are presented in Figure 2.8 for a range of shaft speeds. Reductions of $14-46 \%$ are possible though the highest values here (e.g., $46 \%$ reduction) are for low speed conditions. Over the entire range of speeds measured here, reductions around $20-25 \%$ were the average. These results confirm that significant power loss reductions can be expected for a variety of gas-expanded lubricant formulations when compared to both petroleum-based lubricants and synthetics alone.

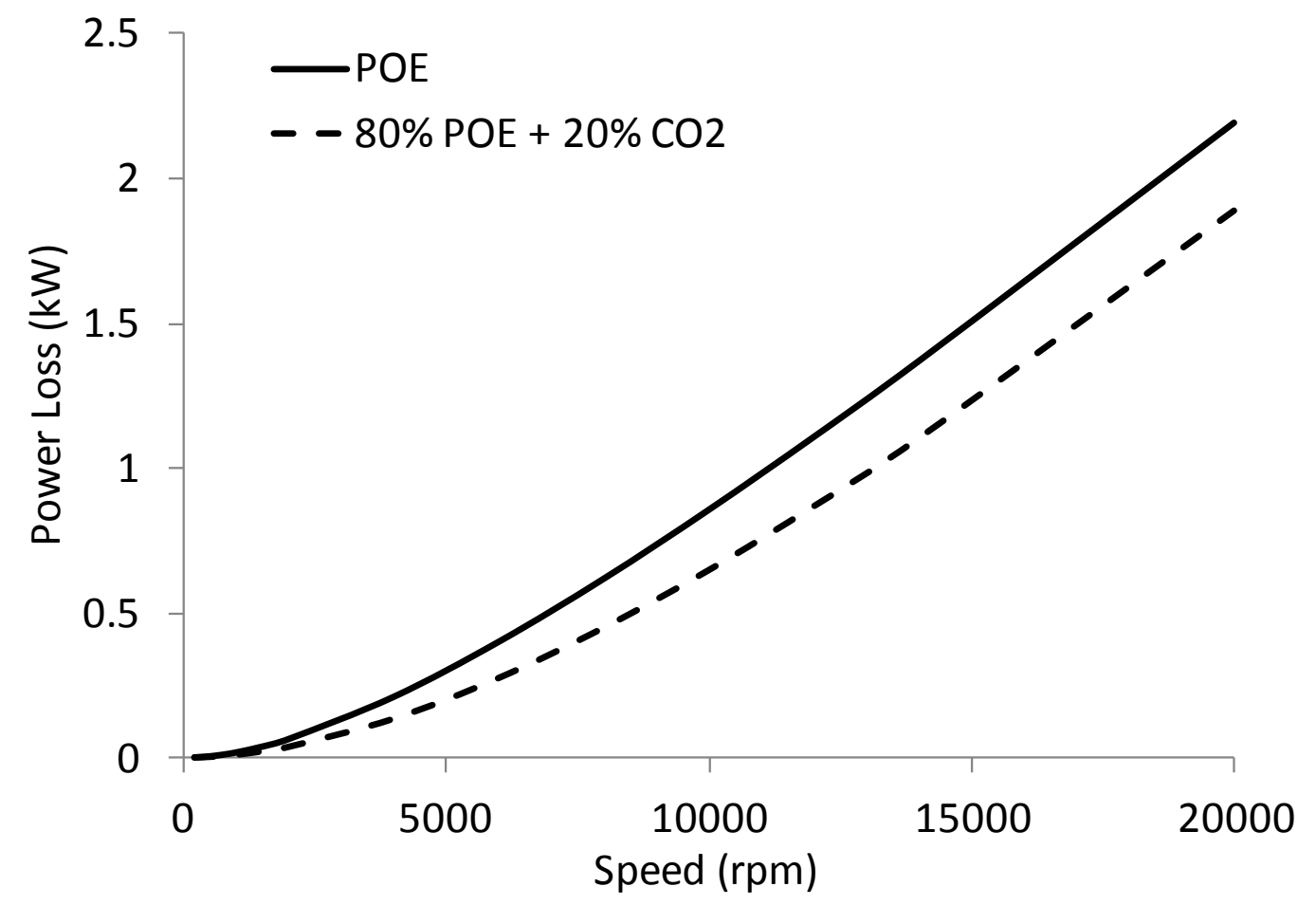

Figure 2.8. Power loss as a function of speed.

Ensuring proper rotordynamic performance is another key element in the implementation of the GEL technology. One important performance measure is the eccentricity ratio of the rotor being supported by the bearing. Figure 2.9 shows the 
eccentricity ratio for both the pure POE and GEL cases. Synchronously reduced stiffness and damping coefficients, shown in Figure 2.10, are significantly lower when the bearing is operated using a GEL. There are competing effects with this change in equivalent stiffness and damping values. While the damping coefficients are reduced, the corresponding reduction in stiffness coefficients can result in greater effective damping of the unbalance response due to increased rotor motion at the bearings [14]. However, this effect is specific to the supported rotor. These bearing results indicate, however, that the bearing could be suitable for support of a flexible rotor while also increasing the energy efficiency of the bearing in cases where the specific rotordynamic characteristics, e.g., the shaft loading, are favorable. Chapter 3 will address the effect of the GEL lubricant on the natural frequencies, unbalance response, and stability of a flexible rotor.

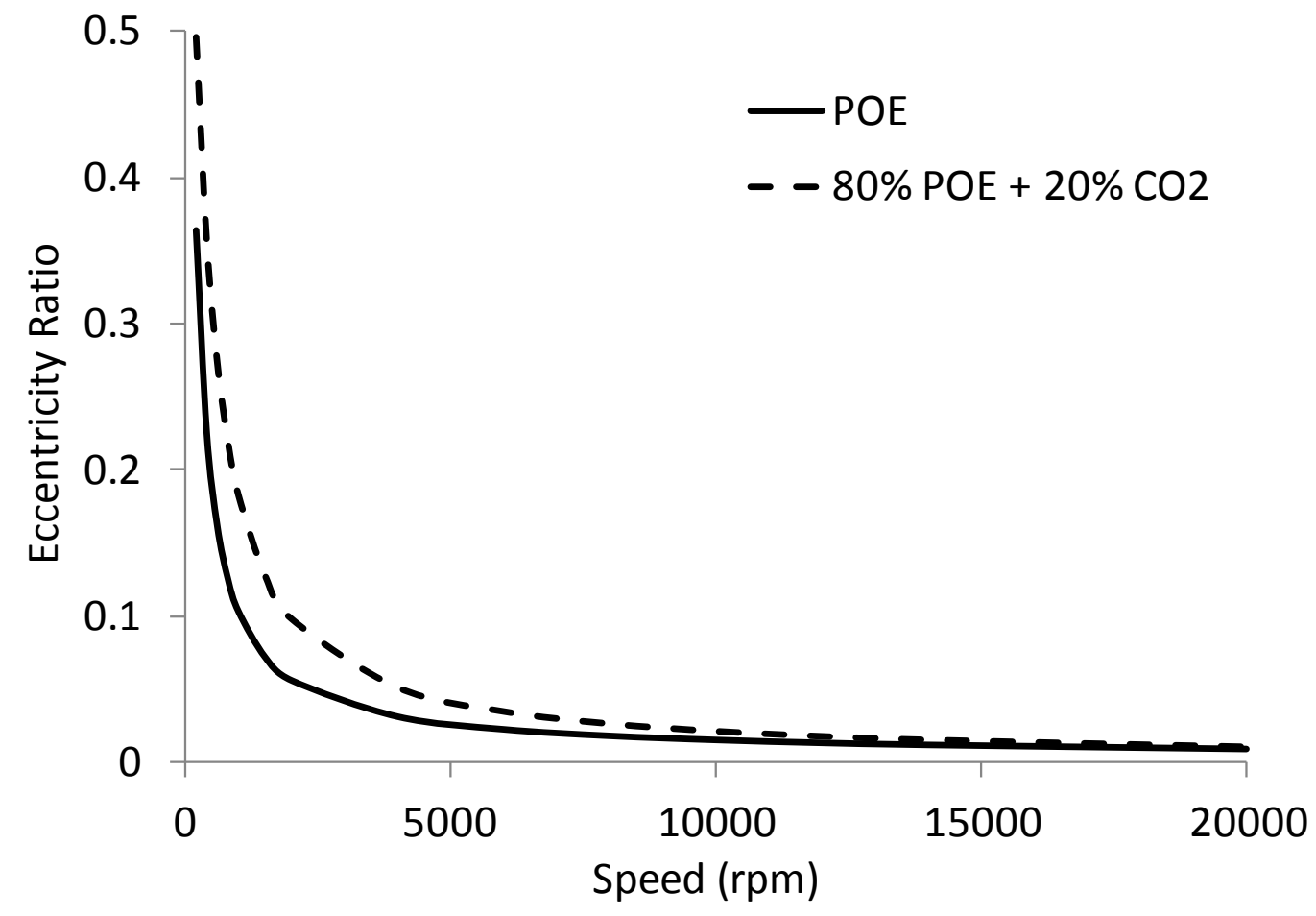

Figure 2.9. Journal eccentricity ratio as a function of speed. 

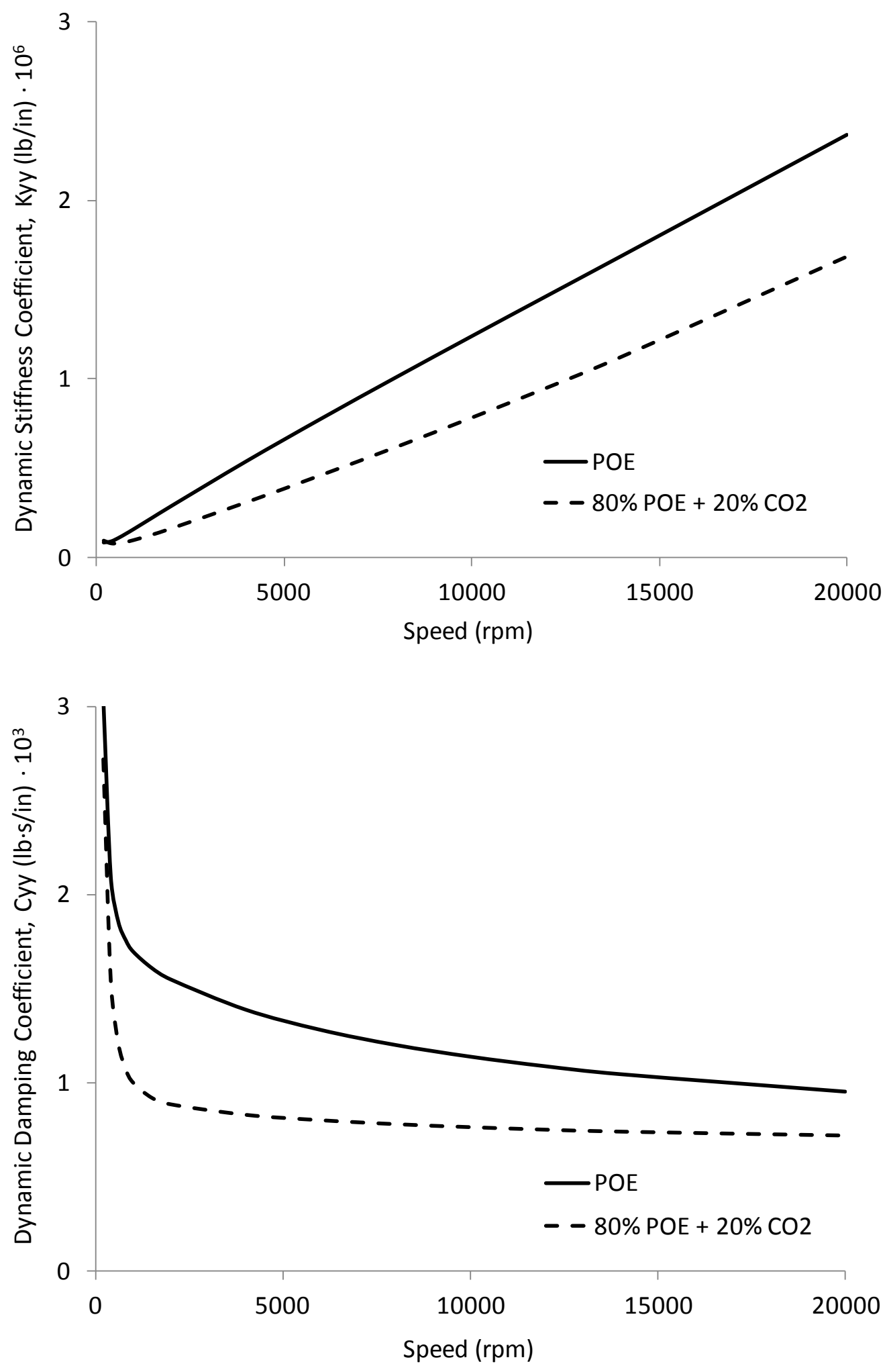

Figure 2.10. Synchronously reduced stiffness and damping coefficients as a function of speed. 
Minimum film thickness, another key parameter of bearing performance directly related to eccentricity ratio, is reported in Figure 2.11 for the two lubricant cases. The GEL-lubricated scenarios result in adequate film thickness except at very low speeds (e.g., $<200 \mathrm{rpm}$ ), where the film thickness is just below the typical minimum threshold of $10^{-5} \mathrm{~m}$ [85]. It is unlikely that a bearing operating under these conditions would use a GEL with a high mass fraction of $\mathrm{CO}_{2}$. For speeds above 4,000 rpm the GEL actually provides for a thicker lubricant film than conventionally lubricated bearings, providing the benefits of better load support as well as greater bearing efficiency.

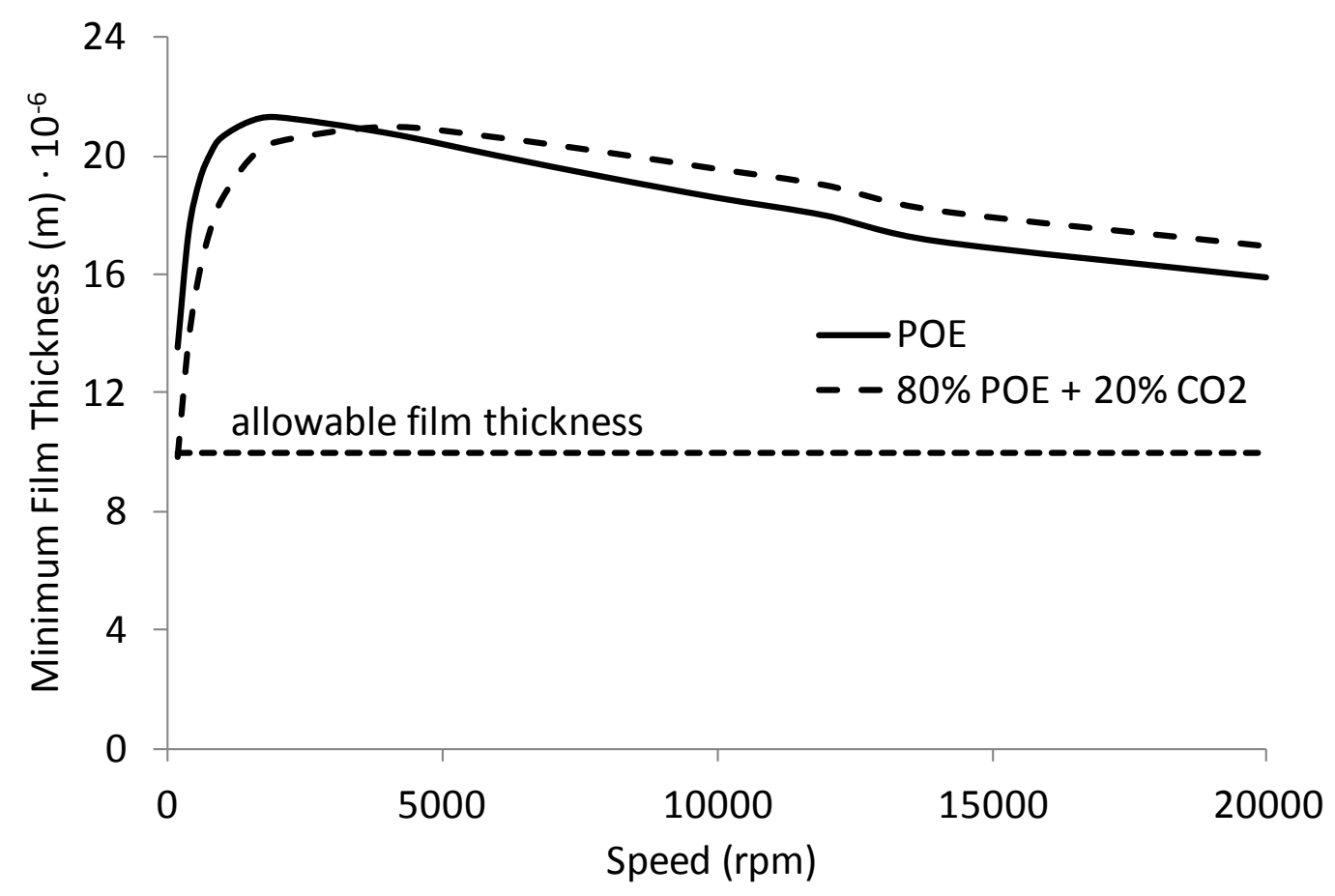

Figure 2.11. Minimum film thickness as a function of speed.

\subsection{Conclusions}

The formation of gas-expanded lubricants was analyzed using experimental and modeling techniques of high-pressure phase behavior and bearing performance to understand the benefits and potential challenges associated with deploying this 
technology. The diffusion of carbon dioxide into synthetic lubricants was found to be a function of the lubricant viscosity as previously demonstrated for other gas-liquid mixtures while the viscosity effects of introducing $\mathrm{CO}_{2}$ were highly consistent across a range of lubricant classes. GEL viscosity for a variety of base lubricants of all viscosity grades was shown to be dependent on the mass fraction of the carbon dioxide present in the system.

The TEHD modeling results suggest that in spite of the higher pressures inside the bearing, any pressure drop experienced during GEL lubrication will be comparable to standard lubricated bearings. Modest levels of phase separation are expected as the fluid exits the region of support under low speed operating conditions but the $\mathrm{CO}_{2}$ and lubricant are rapidly reintegrated before reaching the end of the bearing pad. Even if the phase separation persists, this behavior would not be detrimental to the bearing or to the ability of the fluid to support the bearing load. Furthermore, any thermal expansion is considered to be negligible, with lower overall operating temperatures also expected in a GEL lubricated bearing, resulting in lower rates of wear in the bearing and a long-term cost savings for the user. Other rotordynamic performance parameters, such as eccentricity and minimum film thickness, are well within the acceptable range for GEL lubricated bearings, though significant effects on synchronously reduced stiffness and damping coefficients were found. The improvements in system efficiency are appreciable and these unique and tunable properties offered by GELs make them a promising approach for improving the energy efficiency of rotating machinery. 


\section{Chapter 3}

\section{Gas-Expanded Lubricant Performance and}

\section{Effects on Rotor Stability in Turbomachinery}

\subsection{Introduction}

In this work, the bearing and rotordynamic performance of two representative highspeed machines was evaluated when different lubricants, including GELs, were supplied to the machine bearings. While past work by the author has suggested that GELs could enhance bearing performance when compared to conventional fluids in flooded journal bearings, these results have given rise to a number of questions that need to be further evaluated before the technology can be deployed. Chief among these questions is how the use of a tunable fluid will affect the stability margin in high-speed rotating machines. GELs have been shown analytically to produce lower stiffness and damping coefficients in lightly loaded bearings as well as lower coefficients at higher speeds in more heavily loaded bearings when compared to standard fluids, but it is unclear how these changes in bearing behavior will affect the overall stability of the machines that these bearings

*Originally published by ASME. See reference [116]. 
support. In implementing the GEL technology, ensuring that the tunable nature of these fluids will not negatively impact the rotor stability will be critical. The knowledge related to the range of rotor behavior that is expected as a function of lubricant composition will also demonstrate the potential for rotordynamic control over these machines using these fluids.

While lubricant properties are common inputs to models designed to analyze bearing performance, there are few studies that have focused primarily on the effects that these properties have on bearing and rotordynamic performance, and no studies that have extended that performance analysis to fluid mixtures with unique properties such as GELs. Glavatskih et al. [10] examined the significance of lubricant thermal properties on tilting-pad thrust bearing performance and found that replacing an ISO VG 68 mineral oil with an ISO VG 46 ester-based synthetic fluid reduced power loss and operating temperature while providing a comparable oil film. They also found that viscosity index had the most significant effect on bearing performance, notable because synthetic fluids typically have a higher viscosity index relative to petroleum-based lubricants. A later study by this group [86] confirmed these results, presenting some of the benefits of less viscous fluids and synthetic fluids over their more viscous counterparts, though both studies were limited solely to bearing performance. Flack et al. [87] examined the effects of lubricant viscosity on rotor instability threshold using standard fluids and an experimental test rig consisting of a three-mass flexible rotor and multiple bearing designs. They concluded that decreasing the lubricant viscosity via inlet temperature increases could either increase or decrease the instability threshold, depending on the lubricant used and the bearing design considered. Singhal [88] also noted this behavior in 
a simple thermohydrodynamic analysis, finding that journal bearing systems could be stabilized by either heating or cooling the lubricant, depending on the operating region.

Studies on fluid mixtures and other non-conventional lubricants have focused primarily on multi-phase mixtures and non-Newtonian lubricants. Grando et al. [41] studied two-phase (liquid-gas) flow in journal bearings, considering the effects of mixtures of oil and refrigerant on journal bearing behavior and cavitation. They found that under an equilibrium boundary condition, the gas would dissolve into the fluid in the rotor support region, resulting in no detrimental effects on bearing performance. Under a non-equilibrium boundary condition, where two-phase flow is present throughout the bearing geometry, it was found that under moderate loading conditions the two-phase fluid film would provide adequate support for the applied load while also reducing eccentricity and friction due to the compressibility of the gas. Roach and Goodwin [89] studied vibration control experimentally using aerated lubricants on a journal bearing test rig. Their results showed that stability can be increased by these lubricants due to reduced bearing stiffness. This may not necessarily be the result for all machines, though, as demonstrated by an analysis performed by Younan et al. [43], which showed air entrainment in squeeze film dampers resulted in increased vibration and reduced load capacity. Samantaray et al. [90] investigated the effect of two-phase (liquid-solid) lubricants on bearing stability and found that these fluids have the potential to provide stabilizing, spring-like forces in journal bearings to control whirl orbit size. A number of studies have also been published evaluating the effects of non-Newtonian, viscoelastic lubricants on bearing stability [91, 92], load capacity [93], and minimum film thickness [94]. 
The goal of this study is to begin answering the critical questions surrounding the overall effects of the GEL technology on rotating machines by analyzing these effects in two representative case studies. The case studies will be presented by first modeling bearing behavior under varying lubrication conditions, comparing standard petroleumbased lubricants with polyol ester (POE) synthetic oils and POE-based GELs. Stiffness and damping coefficients predicted from this analysis will then serve as inputs to rotordynamic finite element models for predicting the rotordynamic behavior of the two machines studied: an eight-stage gas reinjection centrifugal compressor and a four-rotor steam turbine-generator system. These machines are promising candidates for the GEL technology and represent a wide range of speed and loading conditions, many of which that have not yet been analyzed for GEL performance. The results of these case studies will be discussed along with operational considerations and the potential effects of GEL bearing seals on system performance.

\subsection{Modeling Framework}

The modeling approach used in this study consisted of a two-step process of analyzing bearing performance and its subsequent effects on machine rotordynamic performance. The first step involved modeling the behavior of the bearings, considering a number of bearing performance metrics including power loss, minimum film thickness, eccentricity, and stiffness and damping coefficients. The TEHD finite element model described in section 2.3.2 was used to perform this step of the analysis. Stiffness and damping coefficients were calculated and were then used as inputs, in the second step, to finite element models for determining the rotordynamic behavior of the two machines 
considered, allowing for an analysis of the potential effects of different lubrication scenarios on rotor unbalance response and stability.

\subsubsection{Fluids Analyzed}

The lubricants analyzed for their effects on bearing-rotor system behavior were a standard ISO VG 32 petroleum-based lubricant, an ISO VG 46 petroleum-based lubricant, two polyol esters (POE), and POE-based GELs. Polyol esters are known to be highly compatible with $\mathrm{CO} 2$, and experimental data is available in the literature detailing the high pressure fluid phase behavior of POE/CO2 mixtures [32]. Lubricants with viscosities comparable to those of an ISO VG 32 grade were modeled in all bearings except for the gearbox bearings in the turbine- generator system, where lubricants near an ISO VG 46 grade are more commonly used. A combination of experimental and published parameters were used as inputs to the bearing model (Table 3.1). Carbon dioxide solubility data was obtained from Hauk [32] for the GELs. Viscosity data was provided by the lubricant manufacturers and was entered into the bearing model at $40^{\circ} \mathrm{C}$ and $100^{\circ} \mathrm{C}$ isotherms. This data was then fit to the Vogel equation to calculate the lubricant viscosity at any temperature locally within the bearing.

$$
\begin{aligned}
& \mu=\mu_{\text {ref }} \mathrm{e}^{\beta\left(\mathrm{T}-\mathrm{T}_{\mathrm{ref}}\right)} \\
& \text { where } \beta=\frac{\ln \left(\frac{\mu_{2}}{\mu_{1}}\right)}{\mathrm{T}_{2}-\mathrm{T}_{1}}
\end{aligned}
$$

The effect of dissolved carbon dioxide on the viscosity of the GELs was measured experimentally with an Anton Paar rheometer as described in [79]. The results were fit using a modified Grunberg and Nissan model, Equation (3.2), which has been used before to accurately predict the viscosity of these mixtures [71]: 


$$
\ln \mu_{\text {Mix }}=m_{1} \ln \mu_{1}+m_{2} \ln \mu_{2}+m_{1} m_{2} d
$$

Table 3.1. Fluid property inputs to the TEHD model.

\begin{tabular}{|c|c|c|c|c|c|c|}
\hline $\begin{array}{l}\text { Fluid Property } \\
\text { Units }\end{array}$ & $\begin{array}{l}\text { Density } \\
\mathrm{kg} / \mathrm{m}^{3}\end{array}$ & $\begin{array}{c}\text { Specific Heat } \\
\mathrm{J} / \mathrm{g} \cdot \mathrm{K} \\
\end{array}$ & $\begin{array}{c}\text { Thermal Conductivity } \\
\mathrm{W} / \mathrm{m} \cdot \mathrm{K}\end{array}$ & $\begin{array}{c}\text { Viscosity } 40^{\circ} \mathrm{C} \\
\mathrm{mPa} \cdot \mathrm{s}\end{array}$ & $\begin{array}{c}\text { Viscosity } 100^{\circ} \mathrm{C} \\
\mathrm{mPa} \cdot \mathrm{s}\end{array}$ & $\begin{array}{c}\text { Supply Pressure } \\
\mathrm{MPa} \\
\end{array}$ \\
\hline ISO VG 32 & 855 & 1.95 & 0.150 & 28.5 & 4.7 & $* 0.1-0.2$ \\
\hline POE 2938 & 945 & 2.30 & 0.164 & 19.0 & 4.4 & $* 0.1-0.2$ \\
\hline $80 \%$ POE $2938+20 \% \mathrm{CO}_{2}$ & 945 & 2.19 & 0.164 & 10.5 & 3.7 & 5.8 \\
\hline ISO VG 46 & 855 & 1.95 & 0.150 & 39.3 & 5.7 & $* 0.1-0.2$ \\
\hline POE 2926 & 1002 & 2.30 & 0.168 & 53.1 & 8.8 & $* 0.1-0.2$ \\
\hline $80 \%$ POE $2926+20 \% \mathrm{CO}_{2}$ & 1002 & 2.19 & 0.168 & 32.2 & 7.4 & 5.8 \\
\hline
\end{tabular}

*The supply pressure of the ISO and pure POE lubricants was 0.1 MPa for the compressor and 0.2 MPa for the turbine-generator system.

The POE thermal conductivity was measured using a Decagon KD2 Pro thermal conductivity meter equipped with a KS-1 sensor for measuring the thermal conductivity of liquids. The density was obtained from the manufacturer of POE 2938 and POE 2926 lubricants (Chemtura, Fords, NJ). The effect of dissolved carbon dioxide on thermal conductivity was found to be negligible [79] and Hauk [32] had previously reported that the effect on density was minor. The properties of the POEs from Hauk [32] and the manufacturer-supplied POEs were considered together and found to be consistent since both had similar chemistry and phase behavior. The specific heat of the pure POE was estimated from values reported by Totten et al. [72] for a polyol ester. The effects of temperature and pressure on pure lubricant thermal properties were assumed to be negligible for the ranges considered in this study as demonstrated in [73]. The Jensen model [74], shown in Equation (3.3), was used to calculate the specific heat of the lubricant-carbon dioxide mixtures.

$$
\mathrm{C}_{\mathrm{P}_{\mathrm{Mix}}}=\mathrm{m}_{\mathrm{CO}_{2}} \mathrm{C}_{\mathrm{P}_{\mathrm{CO}_{2}}}+\left(1-\mathrm{m}_{\mathrm{CO}_{2}}\right) \mathrm{C}_{\mathrm{P}_{\mathrm{Oil}}}
$$




\subsubsection{Thermoelastohydrodynamic Bearing Model}

Section 2.3.2 provides a detailed description of the TEHD model used for this part of the study. This analysis produced full stiffness and damping coefficients for the bearings that are whirl-frequency independent but that consider small perturbations of the shaft position by solving the perturbed Reynolds equation. These full coefficients were used for the 8-stage compressor analysis. The bearing analysis code may also synchronously reduce the full coefficients to each shaft rotational speed which produces sets of 8 bearing coefficients for each speed. This allowed the model to be used to simulate the speeddependent characteristics of the fluid film bearings and how they interact with the entire rotor structure. The synchronously-reduced bearing coefficients were used in the steam turbine-generator analysis.

Table 3.2 outlines the inputs to the compressor bearing analysis. Sommerfeld Numbers for the compressor bearings were 2.4 in the ISO fluid case, 1.7 in the POE case, and 1.0 in the GEL case at operating speed. These values are driven by the reduction in shear stress that is achieved in the POE and the GEL cases. Inputs to the TEHD model for the bearing analyses performed for the turbine-generator system are presented in Table 3.3. Sommerfeld Numbers at operating speed ranged from $0.05-0.13$ in the generator bearings, $1.69-3.12$ in the low speed pinion, $0.43-0.70$ in the high speed pinion, and $0.25-$ 0.70 in the turbine bearings. 
Table 3.2. Inputs to the compressor TEHD analysis.

\begin{tabular}{lcc}
\hline \hline Parameter & Value & Unit \\
\hline Shaft diameter & 127 & $\mathrm{~mm}$ \\
Rotational speed & 6500 & $\mathrm{rpm}$ \\
Bearing radial clearance & 96.52 & $\mu \mathrm{m}$ \\
Pad axial length & 63.5 & $\mathrm{~mm}$ \\
Pad arc length & 60 & $\mathrm{deg}$ \\
No. of pads & 5 & \\
Load orientation & $\mathrm{LBP}$ & \\
Bearing preload & 0.15 & \\
Pivot offset & 0.55 & \\
Bearing specific load & 513.7 & $\mathrm{kPa}$ \\
Oil supply temperature & 40 & ${ }^{\circ} \mathrm{C}$ \\
Oil supply rate & 45.4 & $\mathrm{~L} / \mathrm{min}$ \\
\hline \hline
\end{tabular}

Table 3.3. Inputs to the TEHD analysis for the turbine-generator system.

\begin{tabular}{lccccccc}
\hline \hline Bearing & Generator 1 & Generator 2 & LS Pinion 1 & LS Pinion 2 & HS Pinion & Turbine 1 & Turbine 2 \\
\hline Bearing Type & Fixed Geom & Fixed Geom & Fixed Geom & Fixed Geom Fixed Geom & Tilting Pad & Tilting Pad \\
Shaft diameter $(\mathrm{mm})$ & 225 & 250 & 225 & 225 & 140 & 110 & 110 \\
Rotational speed $(\mathrm{rpm})$ & 1500 & 1500 & 1500 & 1500 & 10778 & 10778 & 10778 \\
Bearing radial clearance $(\mu \mathrm{m})$ & 168.66 & 187.45 & 116.59 & 116.59 & 136.14 & 104.24 & 104.24 \\
Pad axial length $(\mathrm{mm})$ & 169 & 200 & 170 & 152 & 130 & 55 & 55 \\
Pad arc length $(\mathrm{deg})$ & 180 & 180 & 158 & 158 & 157 & 76 & 76 \\
No. of pads & 2 & 2 & 2 & 2 & 2 & 4 & 4 \\
Load orientation & LOP & LOP & LOP & LOP & LOP & LBP & LBP \\
Bearing preload & 0.00 & 0.00 & 0.66 & 0.66 & 0.36 & 0.41 & 0.41 \\
Pivot offset & - & - & - & - & - & 0.5 & 0.5 \\
Bearing specific load $(\mathrm{kPa})$ & 2576.6 & 2223.6 & 395.1 & 442.0 & 3579.8 & 2107.7 & 1892.6 \\
Oil supply temperature $\left({ }^{\circ} \mathrm{C}\right)$ & 40 & 40 & 40 & 40 & 40 & 40 & 40 \\
Oil supply rate $(\mathrm{L} / \mathrm{min})$ & 40.1 & 40.1 & 40.1 & 45.4 & 62.5 & 62.5 & 62.5 \\
\hline \hline
\end{tabular}




\subsubsection{Finite Element Models of Rotordynamic Performance}

Upon processing the performance data from the TEHD bearing model, rotor models were developed for the machines being studied: an eight-stage gas reinjection compressor documented in Barrett [95] and a steam turbine-generator system documented in Wagner [96]. A schematic of the compressor rotor finite element model is shown in Figure 3.1.

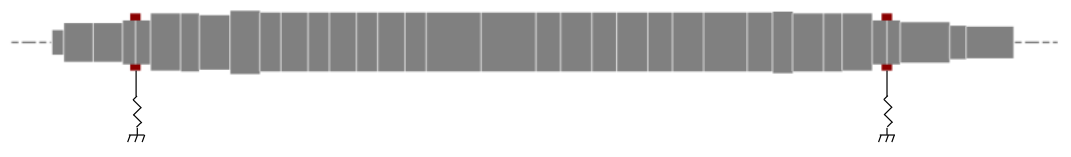

Figure 3.1. Finite element model of the 8 -stage compressor.

The compressor rotor is $2.794 \mathrm{~m}$ in total length with a bearing span of $2.286 \mathrm{~m}$. This length is broken down into 34 Timoshenko beam elements in the finite element model, with the bearings being represented as complex impedances at nodes 4 and 31 . The rotational speed was assumed to be $6,500 \mathrm{rpm}$. The destabilizing mechanism in this rotor system is assumed to be a center piston within the compressor. Destabilizing forces are applied at node 17 to model the effect of the piston on the stability margin. Additional details of the rotor model, including lumped inertias for the coupling half-weight and the impellers, can be found in Barrett [95].

The steam turbine-generator system (Figure 3.2) consists of four rotors and eight bearings of varying size and design, details of which are found in Wagner [96]. The turbine is connected to the high speed pinion of the gearbox via a flexible coupling. A low speed pinion in the gearbox is then rigidly connected to the generator. The complete 
system is defined by 146 elements and 147 nodes. The high speed rotors have a rotational speed of $10,778 \mathrm{rpm}$ and the low speed rotors have an operating speed of 1,500 rpm.

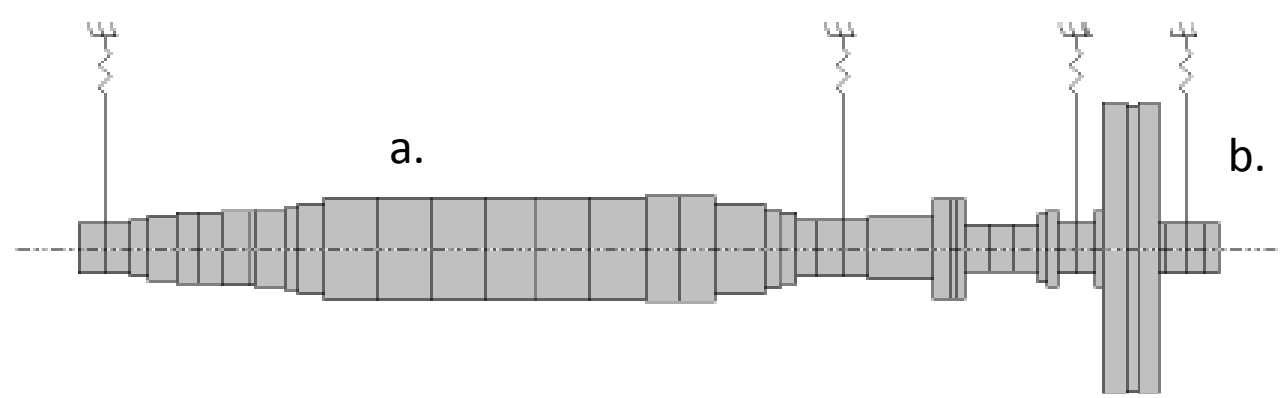

c.

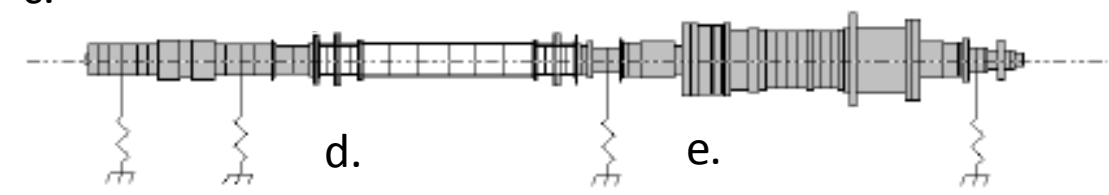

Figure 3.2. Finite element model of the steam turbine-generator system: a. generator, b. low-speed pinion, c. high-speed pinion, d. flexible coupling, and e. steam turbine. Adapted from [96].

The linearized flexible rotor model is represented by the following generalized equation,

$$
M \ddot{u}+(C+\Omega G) \dot{u}+K u=f
$$

containing terms representing the inertia, damping, gyroscopics, and stiffness matrices. Rotordynamic instability, a result of fluid-structure interactions, friction fits, and other effects is fundamentally treated as a free-vibration phenomenon [97]. As such, the stability margin is calculated from the homogenous solution to Equation (3.5), which also includes the cross-coupled stiffness term written explicitly:

$$
M \ddot{u}+(C+\Omega G) \dot{u}+(K+Q) u=0
$$

The cross-coupled stiffness coefficient matrix $Q$ is represented by:

$$
Q=\left[\begin{array}{cc}
0 & q \\
-q & 0
\end{array}\right]
$$


The stability of the rotor is determined using the logarithmic decrement of the first bending critical speed [98]. Damped eigenvalues are obtained in the complex form:

$$
\mathrm{s}=\mathrm{p} \pm \mathrm{i} \omega_{\mathrm{d}}
$$

where $\mathrm{p}$ is the damping exponent and $\omega_{\mathrm{d}}$ is the damped natural frequency. The logarithmic decrement can then be calculated by:

$$
\delta=\frac{-2 \pi p}{\left|\omega_{\mathrm{d}}\right|}
$$

Though commonly used industry specifications such as API 617 [99] require a minimum $\log$ decrement of 0.1 to ensure rotordynamic stability in a manner that considers modeling uncertainty and safety factors, instability was considered to have taken place at values below 0 in this study. API 684 [98] notes that this non-dimensional parameter is a measure of the decay of free vibration, with values greater than 0 indicating a decrease in vibration over time and negative values indicating an increase in vibration over time (rotordynamic instability). These values, along with the calculation of other rotordynamic metrics such as the locations of critical speeds as well as the unbalance response of modes of importance, allowed for a standardized vibration analysis of how changing the fluid properties could impact the overall performance of the machine.

The effects of the gear mesh on the rotordynamic system are represented by a $12 \times 12$ stiffness matrix that couples the axial, lateral, and torsional vibrations of the low and high-speed shafts, originally presented by Stringer [100] and further illustrated by Kaplan et al. [101]. In this analysis, the flexibility of the gear mesh is governed by the stiffness of the teeth only and the gear bodies are assumed to be rigid. Elastohydrodynamic lubrication effects at the tooth contact surface are also neglected. The generalized forces 
and displacements acting along the line of action are then resolved into the global coordinate systems of the low and high-speed shafts. In addition to stiffness considerations, the gyroscopic terms in the equations of motion must be altered to reflect that the low and high-speed shafts operate at different rotational velocities.

\subsubsection{Transient Finite Element Model of Rotordynamic Performance}

To further study the concept of real-time control of bearing and rotor dynamics in high-speed machinery, a time-transient finite element model was also employed to analyze the effects of changing lubricant properties over time on the transient rotordynamic response of the centrifugal compressor. Similar to the steady-state finite element model used in this study, synchronously reduced bearing coefficients were used as inputs to a time-transient finite element model of the rotor. The model utilized was a rotordynamic code described in detail in [102]. With a constant rotating speed $\Omega$, the equation of motion Equation (3.4) is converted to the state space form:

$$
\left[\begin{array}{cc}
\mathrm{M} & 0 \\
0 & \mathrm{M}
\end{array}\right]\left[\begin{array}{l}
\dot{\mathrm{u}} \\
\ddot{\mathrm{u}}
\end{array}\right]+\left[\begin{array}{cc}
0 & -\mathrm{M} \\
\mathrm{K} & \mathrm{C}+\Omega \mathrm{G}
\end{array}\right]\left[\begin{array}{l}
\mathrm{u} \\
\dot{\mathrm{u}}
\end{array}\right]=\left[\begin{array}{c}
0 \\
\mathrm{~F}(\mathrm{u}, \dot{\mathrm{u}}, \mathrm{t})
\end{array}\right]
$$

Let $U=\left[\begin{array}{l}\mathrm{u} \\ \dot{\mathrm{u}}\end{array}\right], \mathrm{A}=\left[\begin{array}{cc}\mathrm{M} & 0 \\ 0 & \mathrm{M}\end{array}\right], \mathrm{B}=\left[\begin{array}{cc}0 & -\mathrm{M} \\ \mathrm{K} & \mathrm{C}+\Omega \mathrm{G}\end{array}\right], \mathrm{F}=\left[\begin{array}{c}0 \\ \mathrm{~F}(\mathrm{u}, \dot{\mathrm{u}}, \mathrm{t})\end{array}\right]$.

Equation (3.9) then has the general state space matrix form:

$$
\mathrm{A} \dot{\mathrm{U}}+\mathrm{BU}=\mathrm{F} \rightarrow \dot{\mathrm{U}}=\left(-\mathrm{A}^{-1} \mathrm{~B}\right) \mathrm{U}+\mathrm{A}^{-1} \mathrm{~F}
$$

Using this form, the second-order ordinary differential equation has been converted to a set of first-order ordinary differential equations that are solved using the explicit fourthorder Runge-Kutta method. 


\subsection{Results and Discussion}

\subsubsection{8-Stage Centrifugal Compressor}

The performance of the centrifugal compressor bearings was modeled at speeds ranging from 500 to $10,000 \mathrm{rpm}$ for three lubricant cases: ISO VG 32, POE, and a POEbased GEL. Reynolds numbers for Pad 2 (Figure 3.3) were the highest encountered in the bearings, with laminar flow being predominant for a majority of speeds in the ISO VG 32 and POE cases. Transitional flow was predicted in these fluid cases only at speeds above the operating speed, whereas it was predicted at speeds above 5,000 rpm in the GEL case. The power loss in the bearings (Figure 3.4) was found to decrease with the use of the synthetic fluid and the GEL, with $40 \%$ reductions in power loss shown when comparing the GEL to the petroleum-based fluid at operating speed.

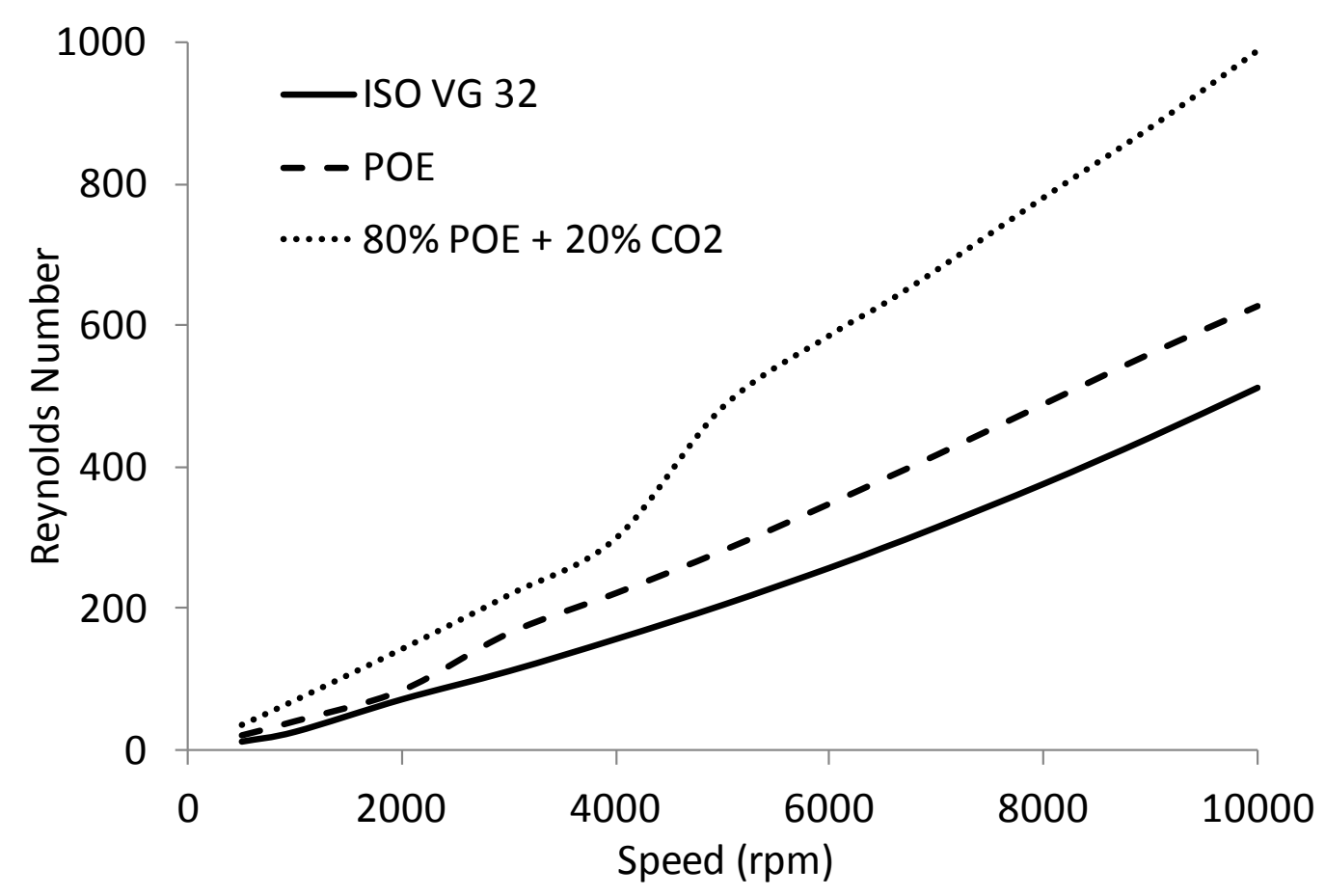

Figure 3.3. Reynolds numbers for Pad 2 in the compressor bearings. 


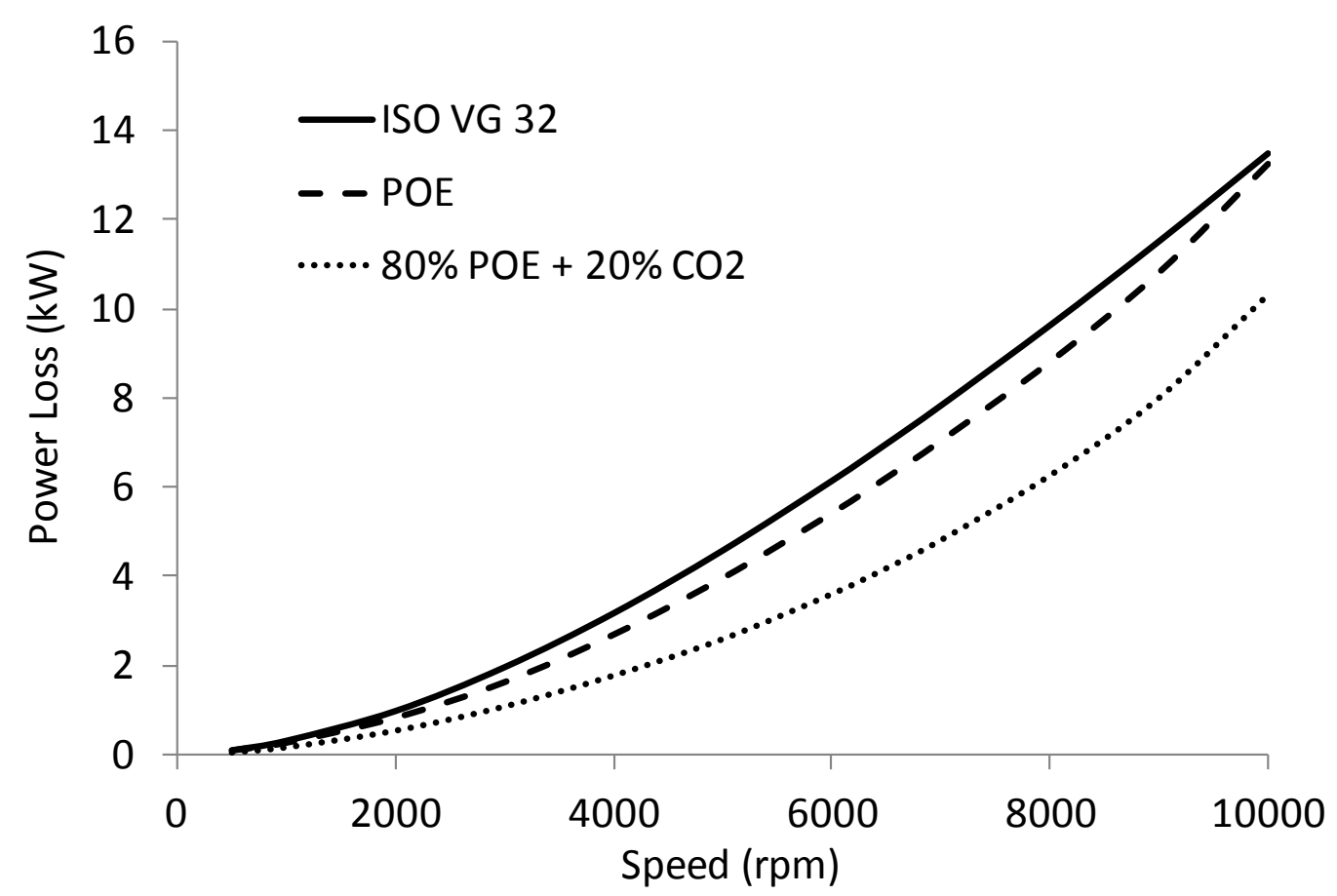

Figure 3.4. Compressor bearing power loss as a function of speed.

Operating temperatures were also found to decrease with the use of GELs due to reduced fluid shear, with fluid film temperatures in the loaded region of the bearing being reduced by approximately $8-12{ }^{\circ} \mathrm{C}$ when comparing the GEL lubricant to the ISO VG 32 lubricant. Reductions in minimum film thickness were also predicted by the model (Figure 3.5), with $15-36 \%$ reductions observed at lower operating speeds, though in all cases the fluid film was found to remain above the typical minimum threshold of $10 \mu \mathrm{m}$ [85]. This behavior is worth noting, however, as reduced film thickness and load capacity could potentially become a limiting factor with the use of less viscous lubricants such as GELs, though reduced temperatures in the fluid film region would likely counteract this effect to a varying extent as well. 


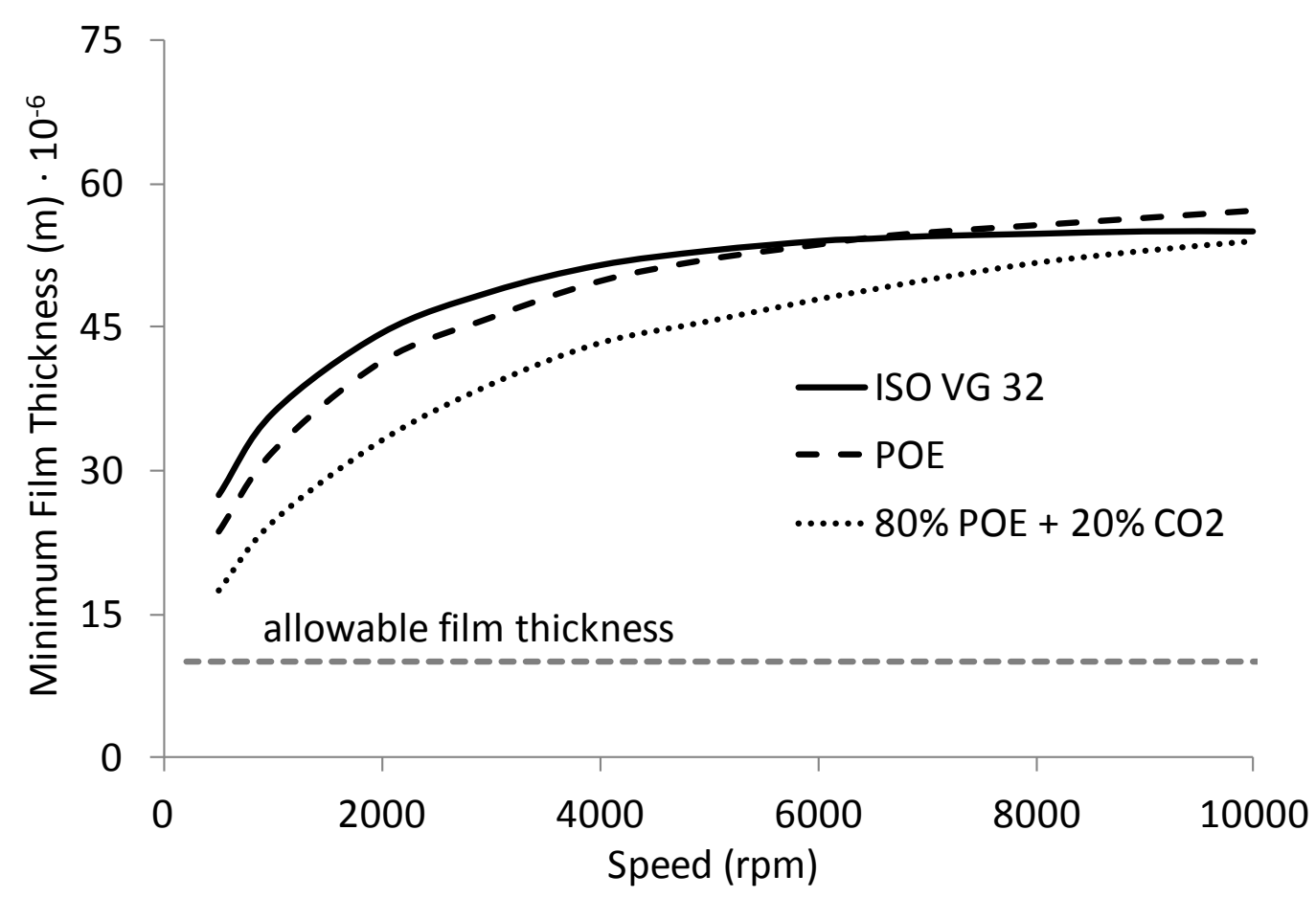

Figure 3.5. Minimum film thickness as a function of speed.

Changes in bearing stiffness and damping behavior due to the use of different lubricating fluids resulted in small increases in bearing eccentricity when comparing the synthetic fluid and GEL to ISO VG 32 as shown in Figures 3.6 and 3.7. This range of possible bearing behavior under varying lubrication scenarios can also result in changes to the locations of critical speeds, shown in Figure 3.7. However, the expected changes in the critical speeds are small with the expected variability in bearing stiffness. This has important implications for operational control of system rotordynamics with GELs as it may be possible to improve the effective system damping for a given mode. It is, however, still necessary to check the effects of other components that affect machine rotordynamics such as seals, which are not modeled here, to more fully understand the full range of expected system behavior. Transient behavior when changing from one set 
of lubricant properties to another must also be considered when studying this operational control.

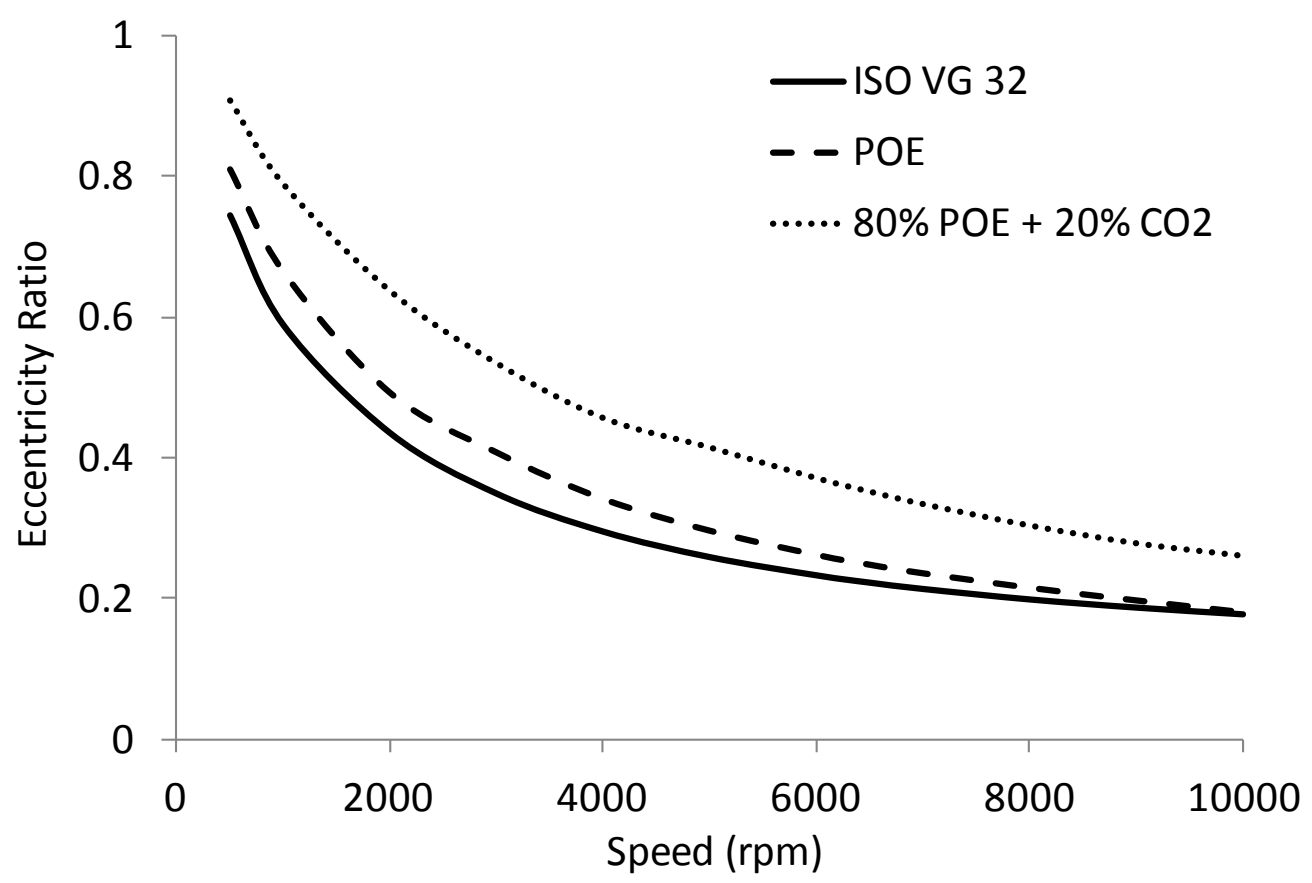

Figure 3.6. Journal eccentricity ratio as a function of speed.

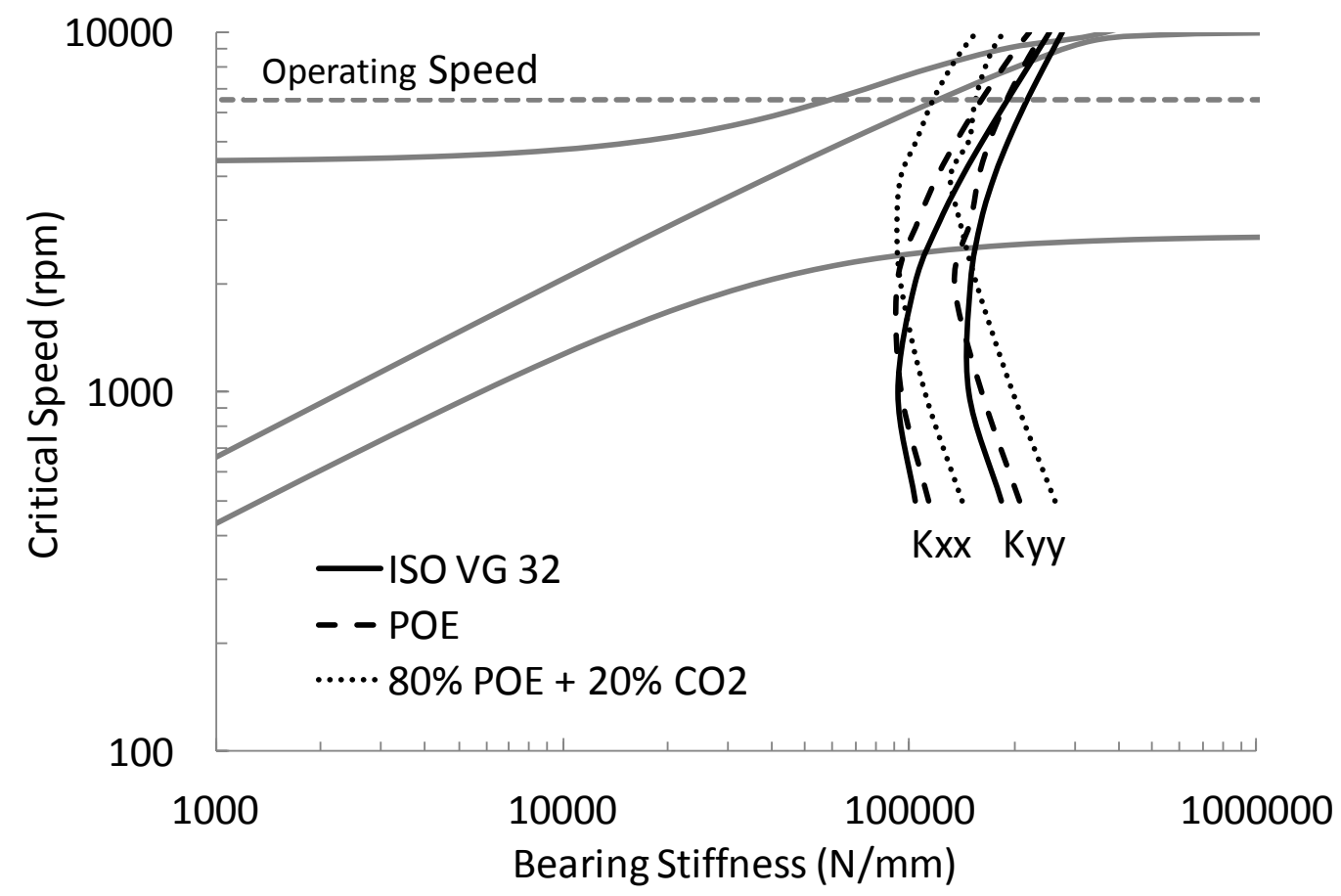

Figure 3.7. Critical speed map of the centrifugal compressor. 
Other important measures of rotordynamic performance, including the unbalance response and stability of the first bending mode, were also analyzed to better understand the effects of different lubrication scenarios on these parameters. The unbalance response due to excitation of the first bending mode, shown in Figure 3.8, was not sensitive to lubricant properties, while the stability margin (Figure 3.9) was improved by the use of the POE and POE-based GEL. The GEL was found to produce the highest stability margin for this mode, showing an increase of $43 \%$ when compared to the ISO VG 32. This was a result of the reduction of direct stiffness and damping coefficients (Figure 3.10 ) by approximately $25-30 \%$ at higher speeds along with $35-40 \%$ reductions in crosscoupled stiffness when comparing the GEL to the ISO VG 32. The second and third modes encountered in this system, conical and gyroscopic modes above the operating speed, respectively, showed similar behavior for the unbalance response. The effect on the stability, however, was counter to the first bending mode as the GEL produced the lowest log decrement values for these modes, though it is noted that for all fluid cases the $\log$ decrement values were substantially higher for these modes than the first bending mode. These results show that the operational flexibility exhibited by the use of tunable fluids may aid in maximizing the stability of a rotordynamic system while not significantly impacting the excitation of rotor critical speeds during machine startup and shutdown. 


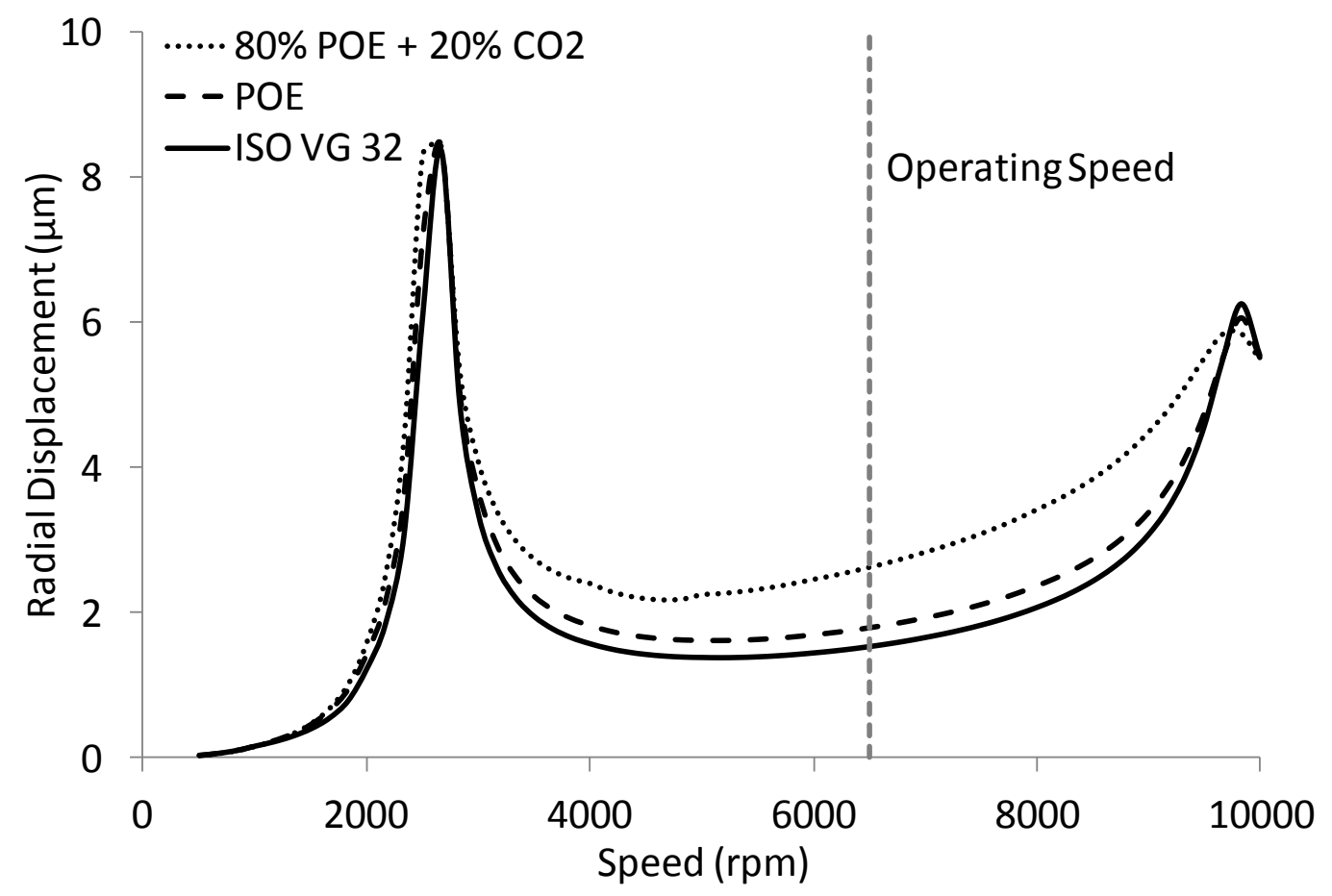

Figure 3.8. Unbalance response of the first bending mode.

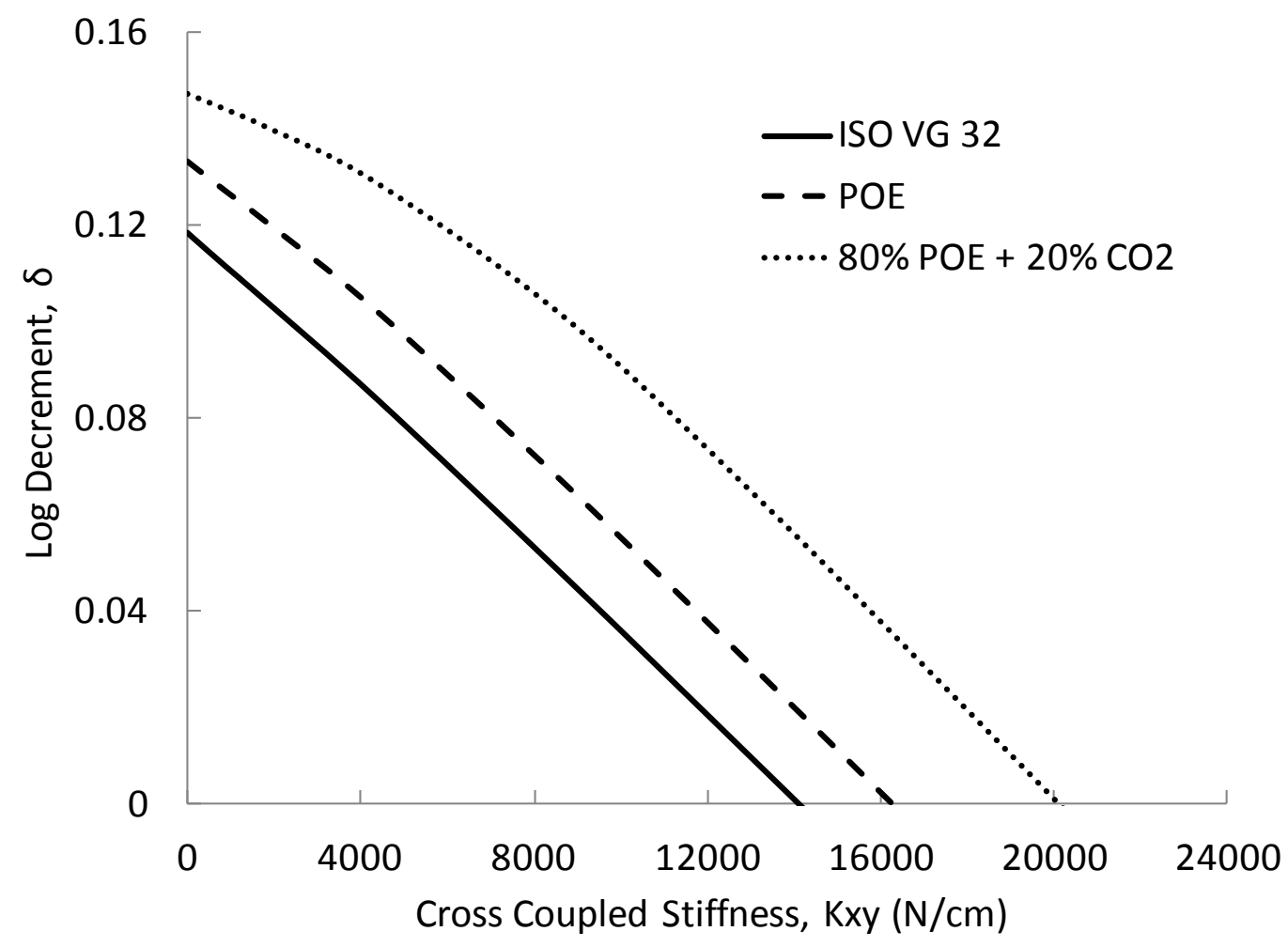

Figure 3.9. Compressor stability margin. 

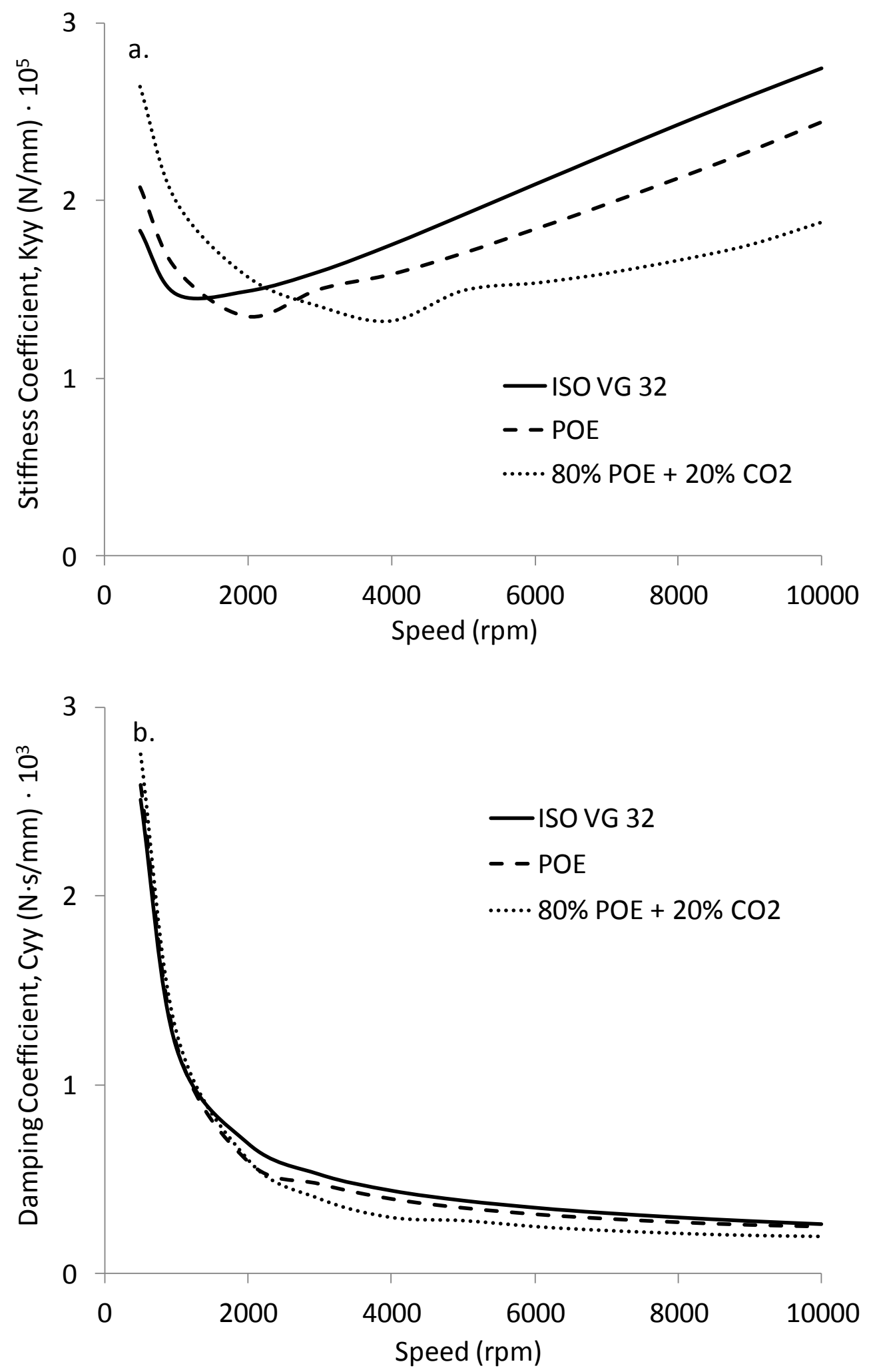

Figure 3.10. Dynamic stiffness (a) and damping (b) coefficients for the compressor bearings. 
As discussed earlier, the transient behavior of the machine must also be considered when transitioning from one set of fluid properties to another. As such, the transient rotordynamic code discussed in section 3.2.4 was used to analyze the transient behavior of the compressor while transitioning between two sets of fluid properties, those of the GEL and the pure POE lubricant. To do this, the residence time of the fluid in the bearing was first calculated using the geometric parameters of the bearing and rotor as well as the lubricant supply rate. This produced a total fluid residence time of 0.35 seconds which was then split into three transitional phases of 0.117 seconds each. The Grunberg and Nissan equation (Equation (3.2) without the $3^{\text {rd }}$ empirical term) was used to calculate the viscosity of the GEL-POE mixtures during these transitional phases. The Jensen model (Equation (3.3) was used to calculate the specific heat of the GEL-POE mixtures while the other fluid properties were held constant. The TEHD model used in this study was used to calculate stiffness and damping coefficients for each of the transitional phases as well as additional values between them to ensure that the trends observed held throughout the viscosity range considered. Combined with the bearing coefficients for the pure POE and GEL lubricants, Figure 3.11 shows the relationship between lubricant viscosity and direct stiffness and damping. This relationship was found to be highly predictable, demonstrating a nearly linear relationship between viscosity and these fluid forces. Crosscoupled stiffness (Figure 3.12) was found to be less predictable overall, though this relationship could still be reasonably predicted as well with an equation fit to the data using simple curve fitting techniques. 

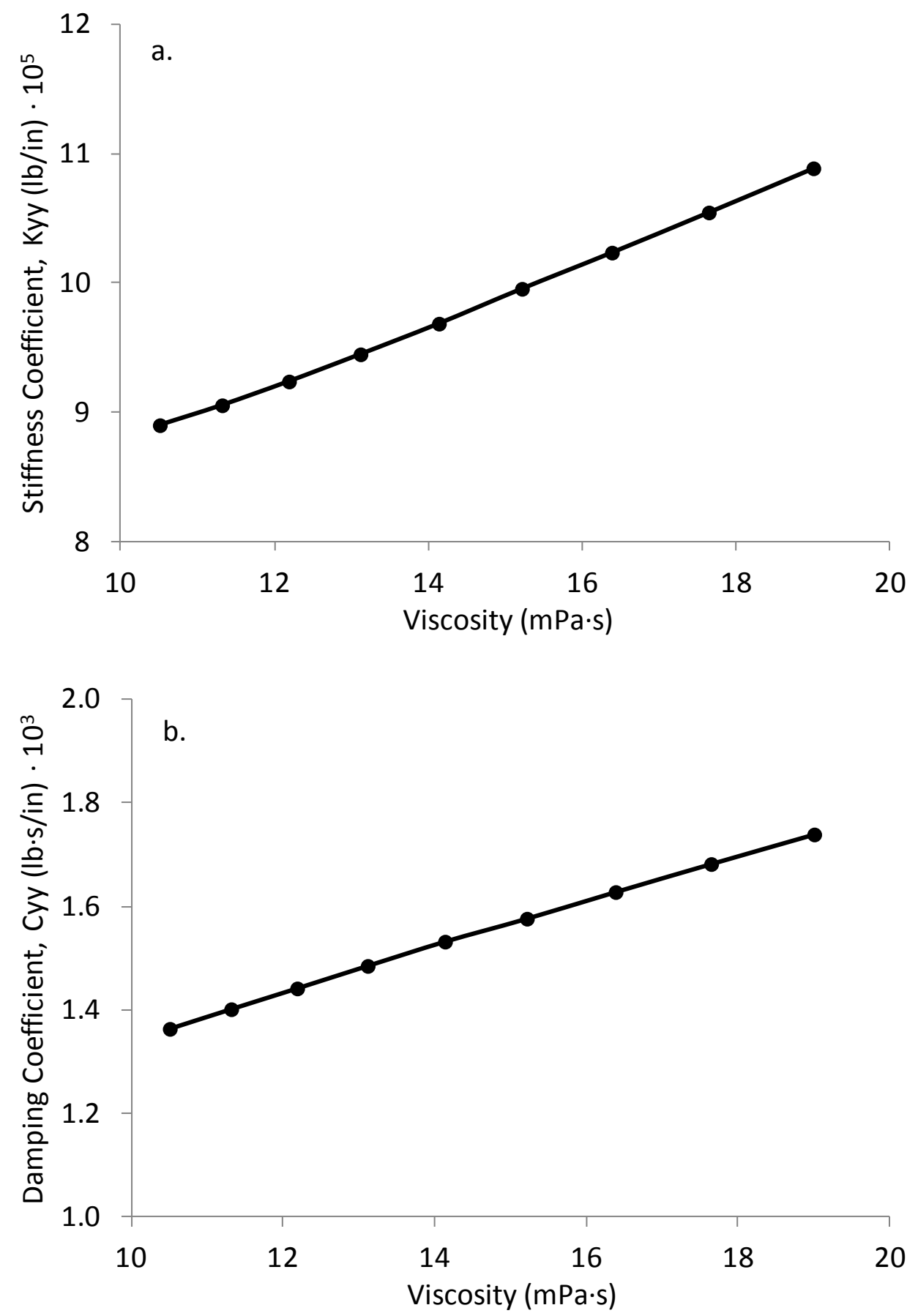

Figure 3.11. Direct a. stiffness and b. damping coefficients as a function of lubricant viscosity. 

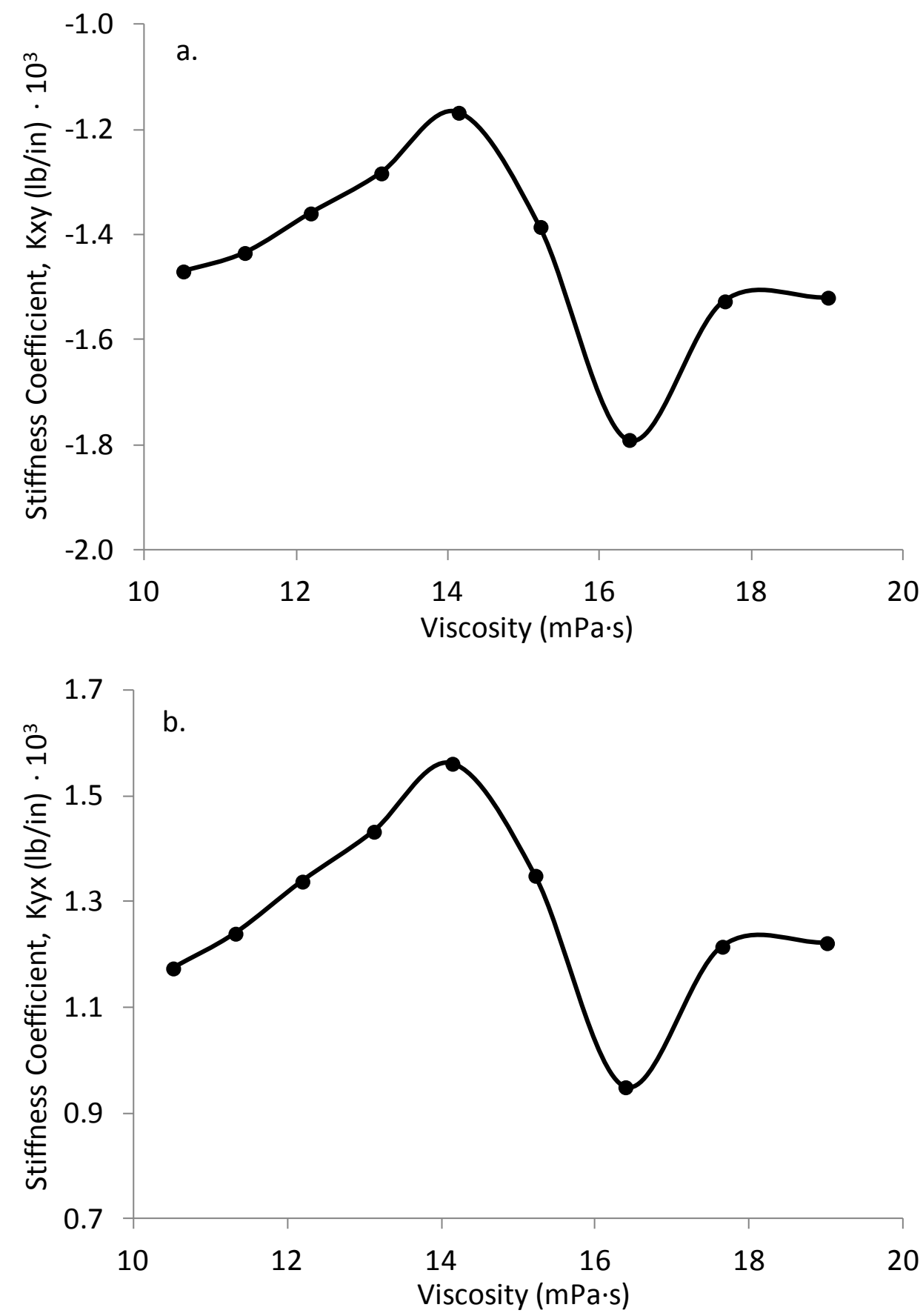

Figure 3.12. Cross-coupled stiffness coefficients as a function of lubricant viscosity. 
Inserting these coefficients at the time intervals discussed above into the transient rotordynamic code, the transient response of the compressor rotor was evaluated. Figure 3.13 shows the transient response of the rotor $\mathrm{x}$ and $\mathrm{y}$ displacements at one of the bearings as the fluid transitions from a GEL to pure POE, then back to a GEL. It can be seen that when using the same scale for the displacements that the majority of change in rotor position was found to occur in the $y$ direction, with the maximum difference between peak displacements found to be 0.0002 ", indicating a relatively smooth transition between the different sets of fluid properties. Mild vibration excitations were also observed at each transition that coincided with the vibration frequency of the $1^{\text {st }}$ bending mode, though these were quickly reduced due to system damping. It should also be noted that if the fluid property shift were modeled more gradually using a transient bearing model that these excitations would likely be reduced if not eliminated. Similar rotordynamic behavior was found at both the second bearing node as well as the central node of the rotor where the unbalance force was applied, with the maximum difference between peak displacements at this node found to be approximately 0.0003 ". 

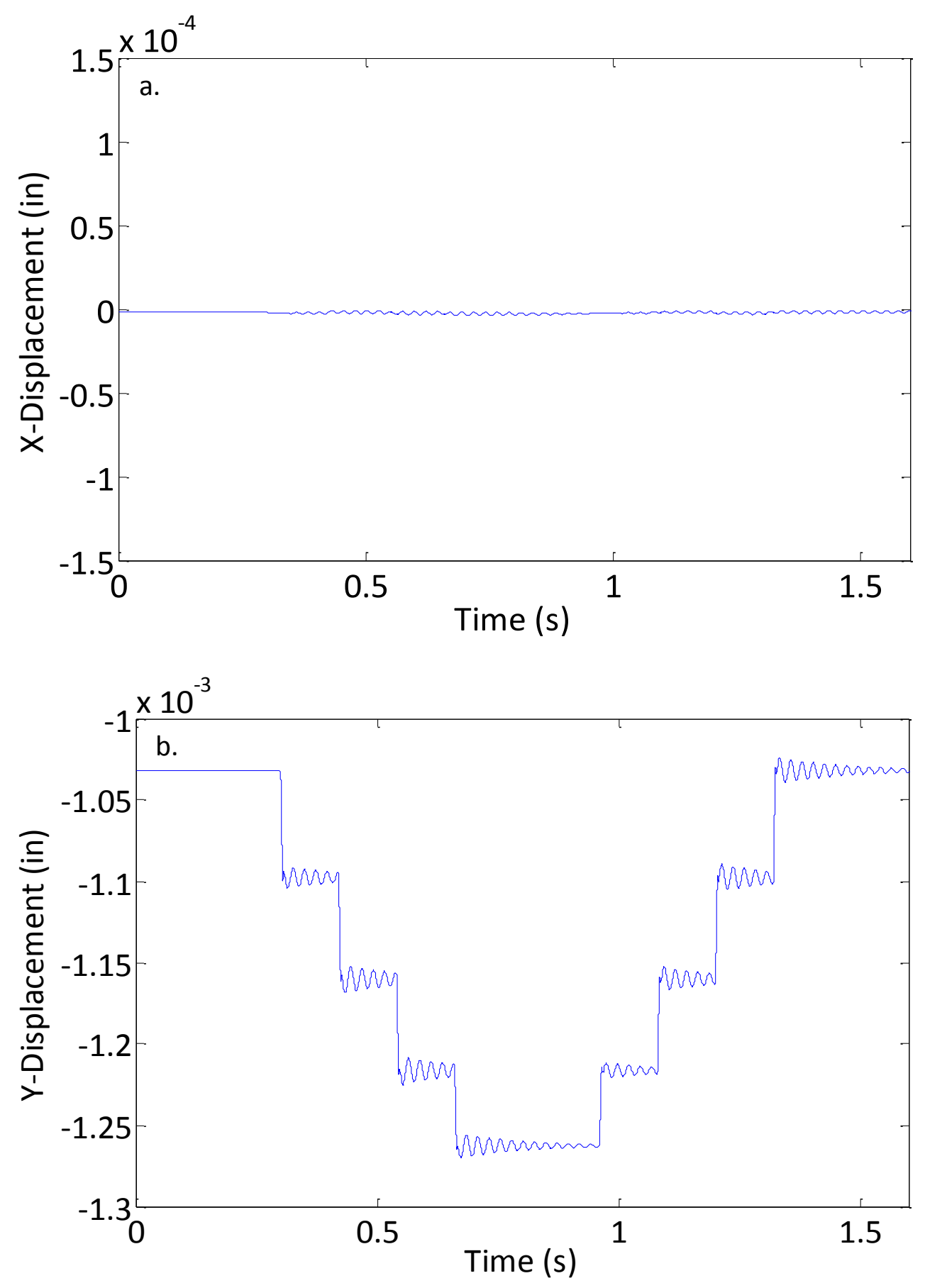

Figure 3.13. Transient $a . x$ and $b$. y displacements of the left compressor bearing. 


\subsubsection{Steam Turbine-Generator System}

The performance of the turbine-generator system was modeled using the same lubrication scenarios and speeds up to $12,000 \mathrm{rpm}$ in the high-speed section, which corresponded to a maximum speed of 1,670 rpm in the low-speed section. The efficiency results for the generator were similar to those of the centrifugal compressor, with 20-23\% reductions in power loss observed when comparing the GEL to the ISO VG 32 at operating speed. Film temperatures in the loaded regions of the generator bearings were also reduced by $10-16{ }^{\circ} \mathrm{C}$. The minimum film thickness was found to be reduced by $32-$ $37 \%$ when comparing the GEL to the ISO VG 32, though film thicknesses remained above the minimum threshold for speeds above $600 \mathrm{rpm}$. Slight increases in bearing eccentricity ratio were also predicted with the use of GELs. Direct stiffness was predicted to be lower in the GEL case at higher operating speeds, though direct damping was found to be increased for a majority of speed cases.

The gearbox bearings for the low and high-speed pinions deviated in behavior from the rest of the machine as the differences in fluid properties were unique for these bearings. While the compressor, turbine, and generator were modeled with an ISO VG 32 and a POE/GEL with lower viscosities than the ISO VG 32, the gearbox bearings were modeled with an ISO VG 46 fluid and a POE with a slightly higher viscosity, though the GEL viscosity was still lower than both the ISO VG 46 and pure POE. In the low-speed pinion bearings this resulted in comparable behavior for the three fluid cases, with the POE and GEL exhibiting slightly higher levels of power loss, thicker lubricant films, and lower eccentricity ratios at higher speeds when compared to the ISO VG 46, though the GEL still produced the lowest film temperatures by approximately $3-9^{\circ} \mathrm{C}$ when compared 
to the petroleum-based fluid. In all fluid cases the flow regime remained laminar in the low-speed pinion bearings.

The high-speed section of the turbine-generator system produced the most interesting results for both the bearing and rotordynamic performance of this machine as the effects of the flow regime became more apparent. These effects were exhibited in the model output, such as in the increase in power loss shown at higher speeds in Figure 3.14. The combination of speed and loading conditions encountered in the high speed pinion resulted in higher overall levels of power loss when compared to the rest of the machine. The onset of turbulent flow at approximately 6,000 rpm in the ISO and POE cases and $10,000 \mathrm{rpm}$ in the GEL case further enhanced these losses, resulting in a $30 \%$ increase in power loss when comparing the GEL to the ISO VG 46 at running speed, though no significant impacts on eccentricity, minimum film thickness, or bearing coefficients were observed due to this change in the flow regime. Another factor contributing to these results were the significant reductions in operating temperature predicted by the model for the GEL case, with fluid film temperatures in the loaded region of the bearing predicted to decrease by $23-30^{\circ} \mathrm{C}$ when compared to the ISO VG 46 . These drastic reductions, while perhaps desirable from a bearing reliability standpoint, produced a counteracting effect on bearing efficiency, with GEL power losses exceeding those of the other fluids for a majority of the speed cases due to an increased lubricant viscosity. 


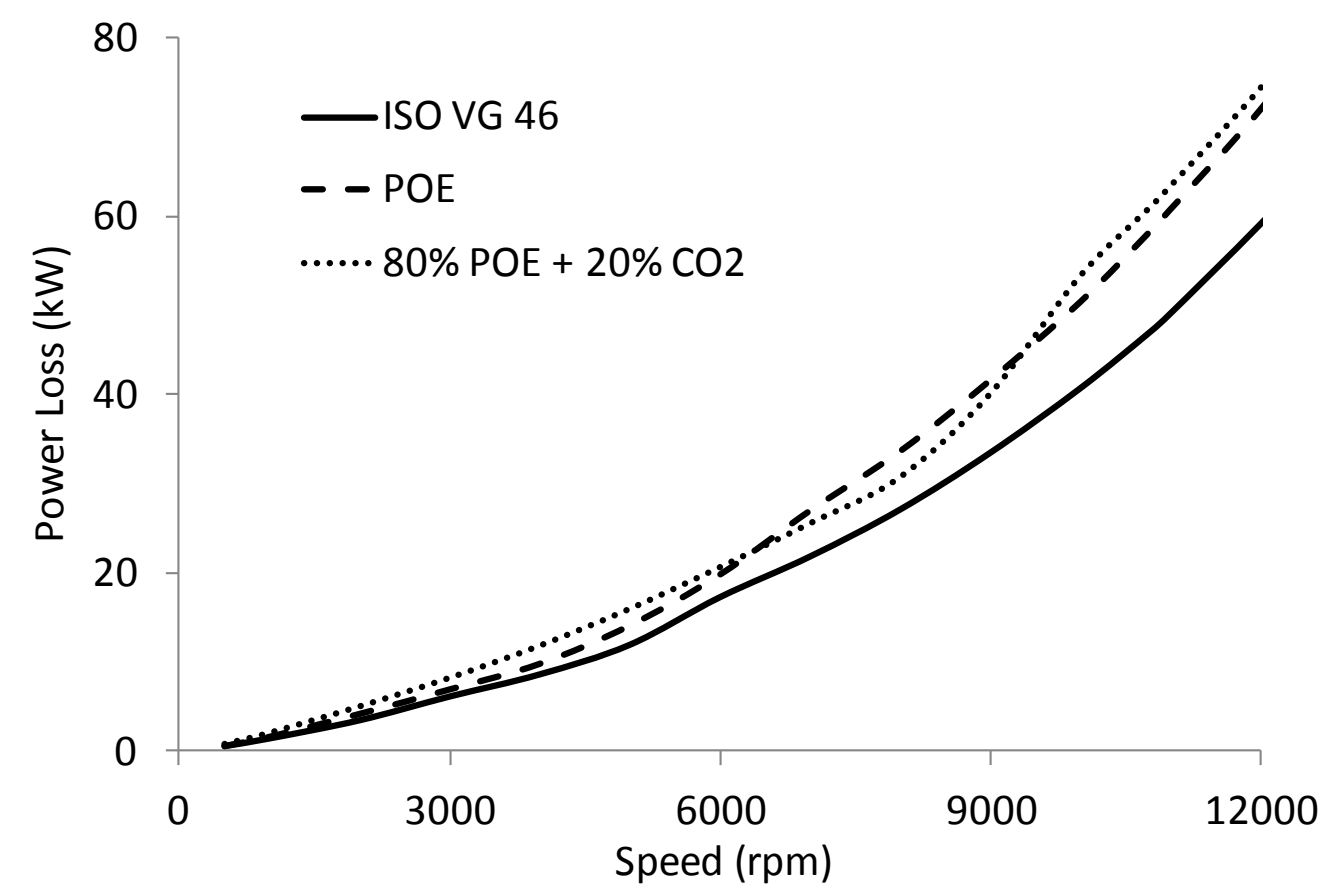

Figure 3.14. Power loss as a function of speed in the high-speed pinion bearings.

In the GEL-lubricated bearings, the significantly lower fluid film temperatures also resulted in a much higher minimum film thickness, shown in Figure 3.15 as being over 3 times thicker at operating speed when comparing the GEL to the ISO VG 46. Bearing eccentricity was shown to be significantly reduced at higher speeds. Direct stiffness and damping coefficients were predicted to be higher for a majority of speed cases in the $\mathrm{x}$ direction but were predicted to be lower in the y-direction. 

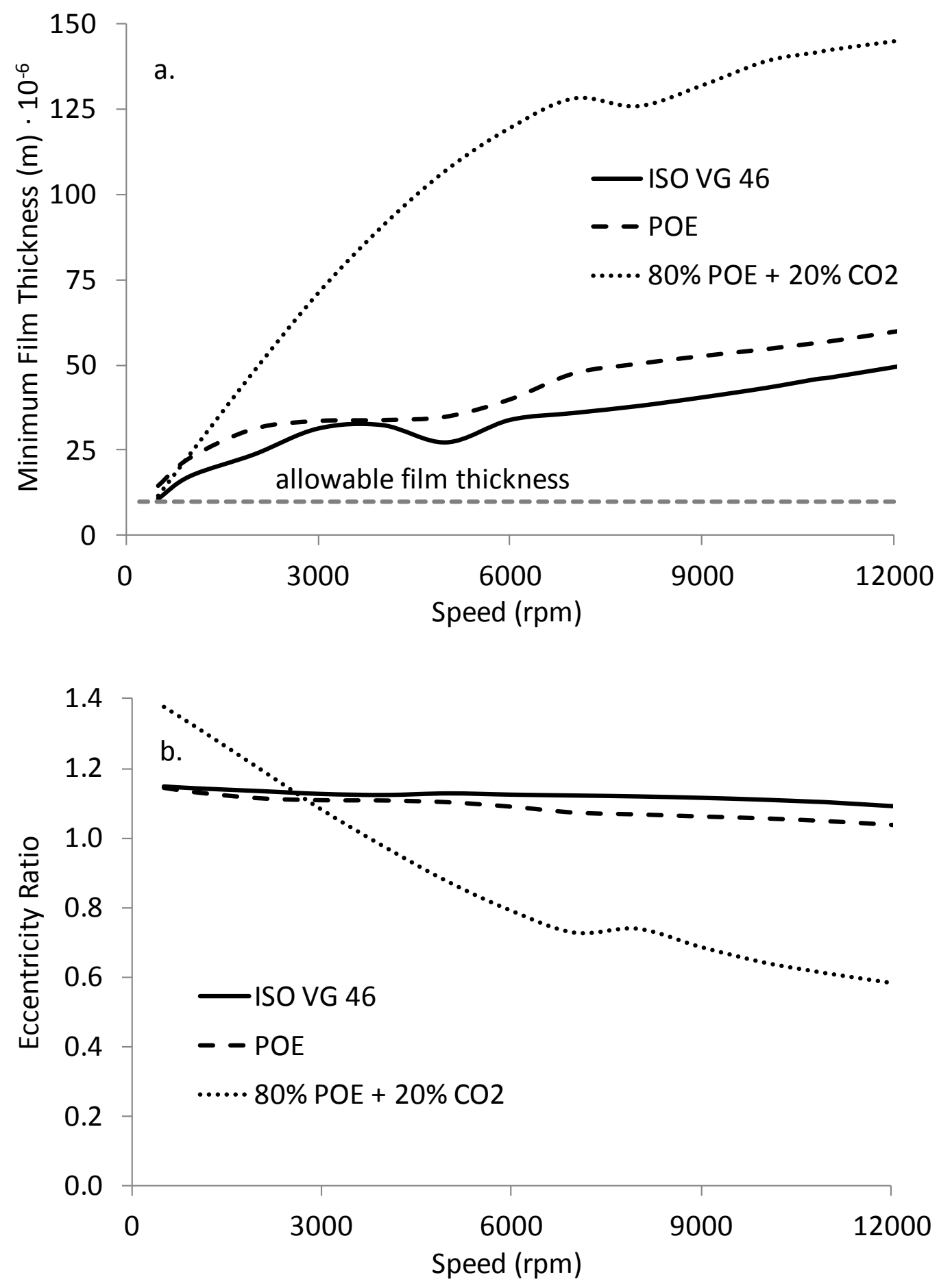

Figure 3.15. Reduced operating temperatures in the high-speed pinion GEL case have significant effects on a. fluid film thickness and b. eccentricity ratio. 
The results of the turbine bearing analysis are comparable to the compressor and generator, though at higher operating speeds the onset of turbulent flow (Figure 3.16) again offsets what would otherwise have been a significant reduction in power loss in the GEL case (Figure 3.17) as the other fluid cases remain in the laminar and transitional flow regimes. Fluid film temperatures were found to be reduced by $8-12^{\circ} \mathrm{C}$ when comparing the GEL to the ISO VG 32 at operating speed, with increases in bearing eccentricity of $18-19 \%$ and reductions in film thickness of $19-20 \%$ also predicted by the analysis, though in all fluid cases the film thickness remains above the minimum threshold for speeds exceeding 2,000 rpm.

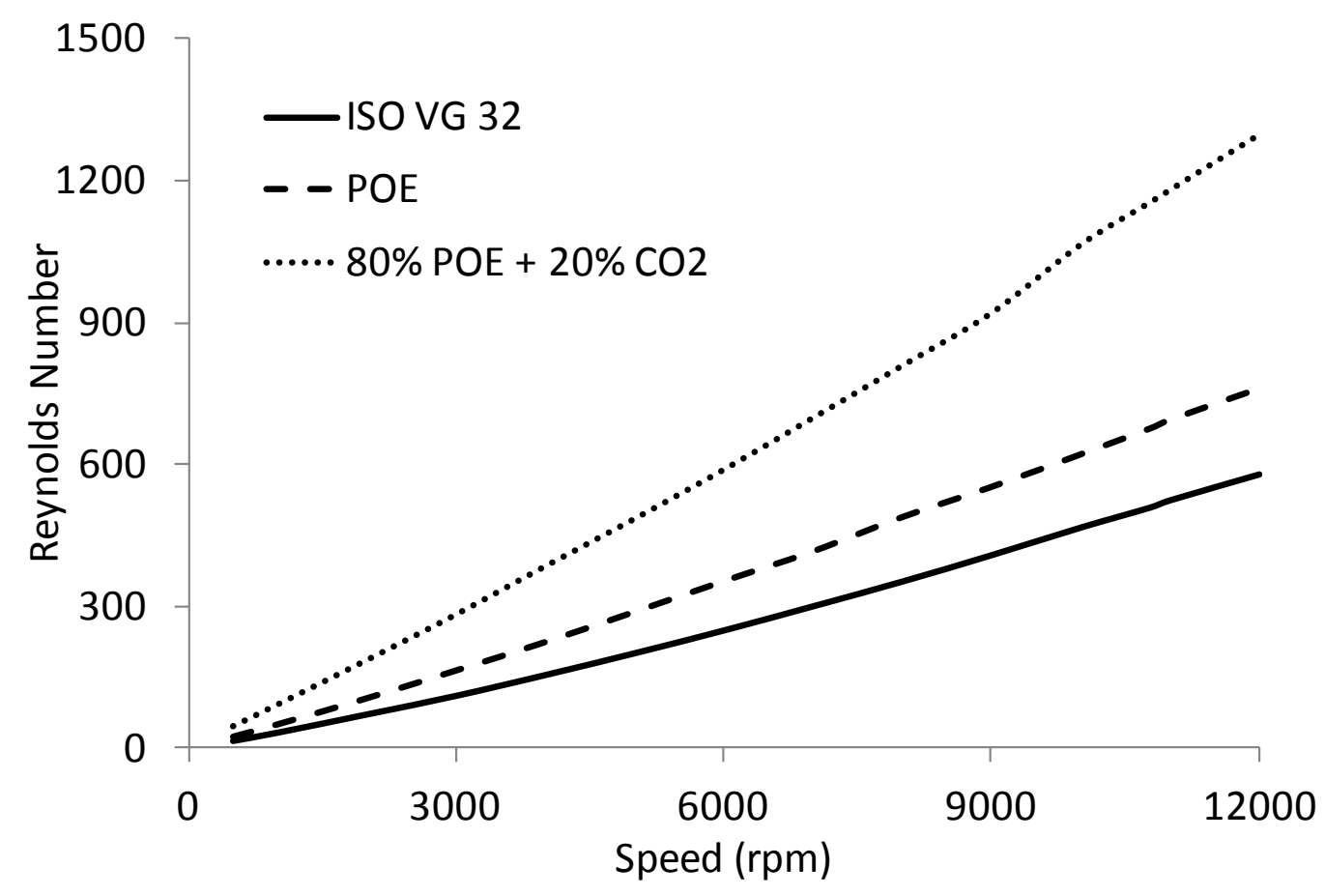

Figure 3.16. Reynolds numbers for Pad 2 in the coupling-end turbine bearing. 


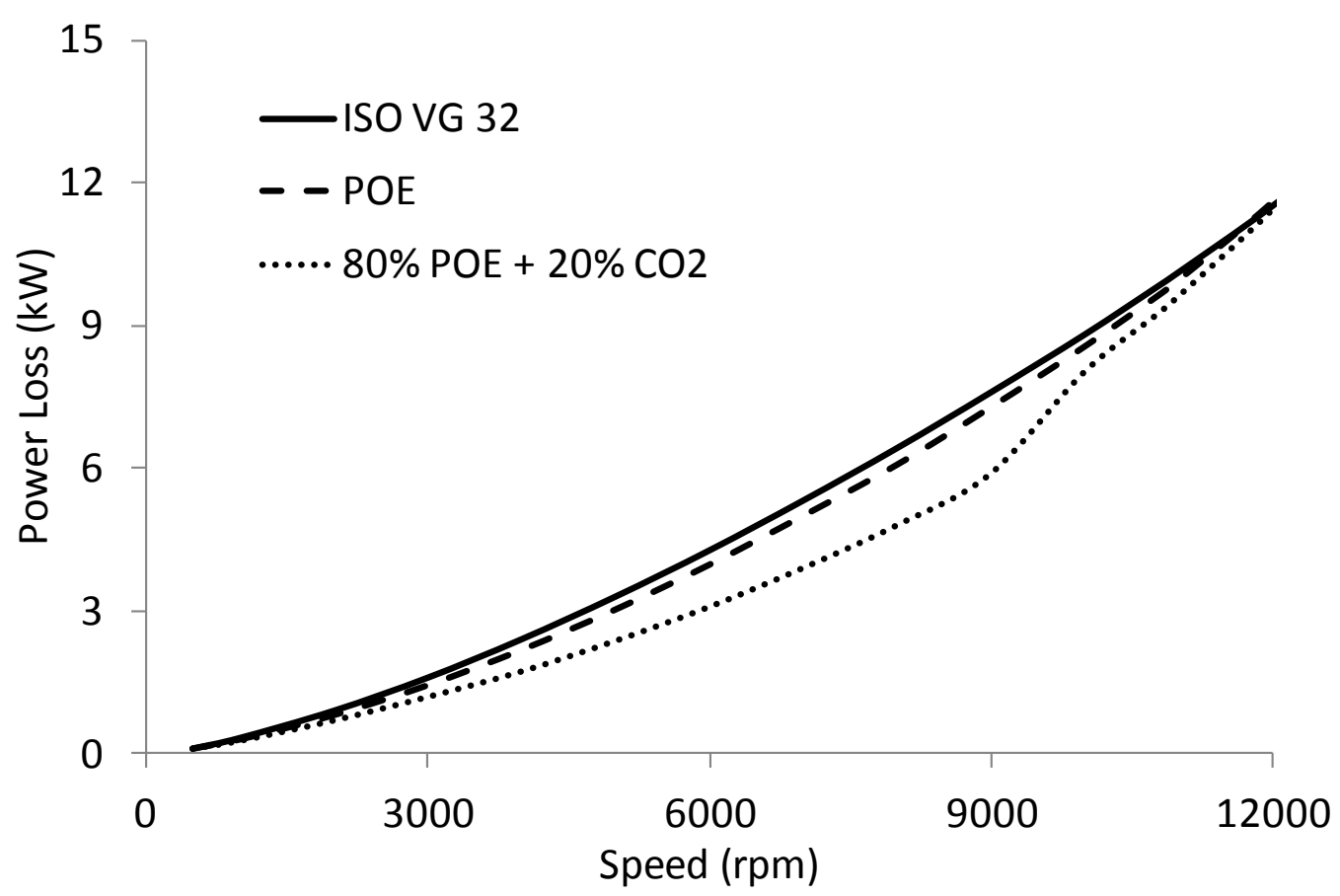

Figure 3.17. Power loss as a function of speed in the coupling-end turbine bearing shows the onset of turbulent flow at 10,000 rpm.

The rotordynamic analysis of the turbine-generator system produced a number of significant findings. The use of different lubricating fluids exhibited a number of effects on the rotordynamics of the system, including the speeds at which the modes were encountered, the mode shapes, the contribution (or lack thereof) of lateral-torsional coupling to the modes, and of greatest concern, the stability of each mode. Table 3.4 presents a complete list of the lateral, torsional, and coupled modes in both the forward and backward directions for the system, showing the speeds at which they occur and their stability listed in terms of log decrement. The damped critical speeds, mode shapes, and $\log$ decrement values between the ISO and POE cases were similar for this rotor-bearing model. In a majority of cases, however, the GELs produced a smaller log decrement than the other fluids. 
Table 3.4. Critical speeds and log decrements for the turbine-generator system. Both forward $(\mathrm{F})$ and backward (B) modes are presented.

\begin{tabular}{|c|ccc|ccc|ccc|}
\hline & \multicolumn{3}{|c|}{ ISO Fluid Case } & \multicolumn{3}{c|}{ POE Fluid Case } & \multicolumn{3}{c|}{ GEL Fluid Case } \\
\hline Mode & $\omega_{\mathrm{d}}$ & Log Dec & Mode Type & $\omega_{\mathrm{d}}$ & Log Dec & Mode Type & $\omega_{\mathrm{d}}$ & Log Dec & Mode Type \\
\hline 1 & NA & NA & NA & 115 & 207.89 & Lat-Tors (F) & 240 & 4120.1 & Lat (B) \\
2 & 513 & -0.0002 & Tors & 513 & -0.0002 & Tors & 513 & -0.0011 & Tors \\
3 & 1081 & 8.9780 & Lat-Tors (F) & 1072 & 9.3465 & Lat-Tors (F) & 852 & 2.3567 & Lat (F) \\
4 & 1200 & 9.8140 & Lat-Tors (F) & 1110 & 10.663 & Lat-Tors (F) & 889 & 1.7689 & Lat (F) \\
5 & 1251 & 5.4840 & Lat-Tors (F) & 1306 & 5.3534 & Lat-Tors (F) & 849 & 0.0729 & Lat-Tors (F) \\
6 & 2038 & 0.9420 & Lat (F) & 2024 & 0.9098 & Lat (F) & 2452 & 0.8814 & Lat (F) \\
7 & 2480 & 0.2077 & Lat (B) & 2484 & 0.1958 & Lat (B) & 2505 & 0.1524 & Lat (F) \\
8 & 4736 & 0.0001 & Tors & 4736 & 0.0001 & Tors & 4736 & -0.00002 & Tors \\
9 & 5330 & 0.0006 & Tors & 5330 & 0.0006 & Tors & 5328 & 0.0001 & Tors \\
10 & NA & NA & NA & 5933 & 9.6410 & Lat (F) & 5582 & 3.3351 & Lat (F) \\
11 & 6130 & 0.5410 & Lat (F) & 6110 & 0.5479 & Lat (F) & 6261 & 0.4340 & Lat (F) \\
12 & 6154 & 0.5342 & Lat (B) & 6142 & 0.5335 & Lat (B) & 6289 & 0.441801 & Lat (B) \\
13 & 6501 & 1.4890 & Lat (F) & 6558 & 1.4701 & Lat (F) & 6524 & 1.9396 & Lat-Tors (F) \\
14 & 7726 & 9.2000 & Lat (F) & 7009 & 9.9956 & Lat (F) & 7841 & 4.1438 & Lat (F) \\
15 & 8218 & 1.2010 & Lat (F) & 8188 & 1.2511 & Lat (F) & 8627 & 0.4565 & Lat (F) \\
16 & 8638 & 0.1400 & Lat (F) & 8643 & 0.1287 & Lat (F) & 8657 & 0.0808 & Lat (F) \\
17 & 11761 & 0.7340 & Lat (F) & 12428 & 0.5122 & Lat (F) & 12264 & 1.0857 & Lat (F) \\
\hline
\end{tabular}

The shape and log decrement of different modes changed more drastically than others for a variety of reasons. The mode shapes are heavily influenced by the bearing stiffness if there is substantial motion at the bearing sites for that mode. Substantial motion at the bearing sites also leads to greater effective damping which increases the log decrement.

Of the modes that could be excited when the machine is operated using GELs, modes 3,4 , and 5 are the most concerning because their stability is reduced quite significantly. These modes primarily affect the vibration of the low-speed shaft and its whirl frequency is within the operating speed range. Therefore, these modes could be excited by crosscoupling forces in the sleeve bearings supporting this shaft [98]. The shapes for mode 5 are shown in Figure 3.18 for the low-speed shaft for the GEL and ISO lubricant cases, respectively. For brevity, modes 3 and 4 are not shown although a similar trend would be discovered. These modes also had large enough log decrements in the GEL case to be 
considered stable. Insights about the effective damping of mode 5 can be determined from these shapes. The GEL case shows little to no motion of the shaft at the generator bearings, but the bulk of the participation is at the low-speed pinion bearings. Despite the GEL lubricant contributing to higher damping coefficients $(86 \%$ higher in the $\mathrm{x}$-direction at operating speed), the lack of motion at the generator bearings impedes their effective damping, which decreases the log decrement of the mode. Also, the analysis results for the low-speed pinion bearings with GEL lubricant indicate some negative principal stiffness coefficients, which are destabilizing. The ISO mode shape shows slightly more modal participation of the generator because of the substantially larger principal stiffness coefficients of the low-speed pinion bearings. Consequently, the ISO case for mode 5 has a much larger log decrement because the principal stiffness coefficients are not only positive, but also because they include lower cross-coupled stiffness. 

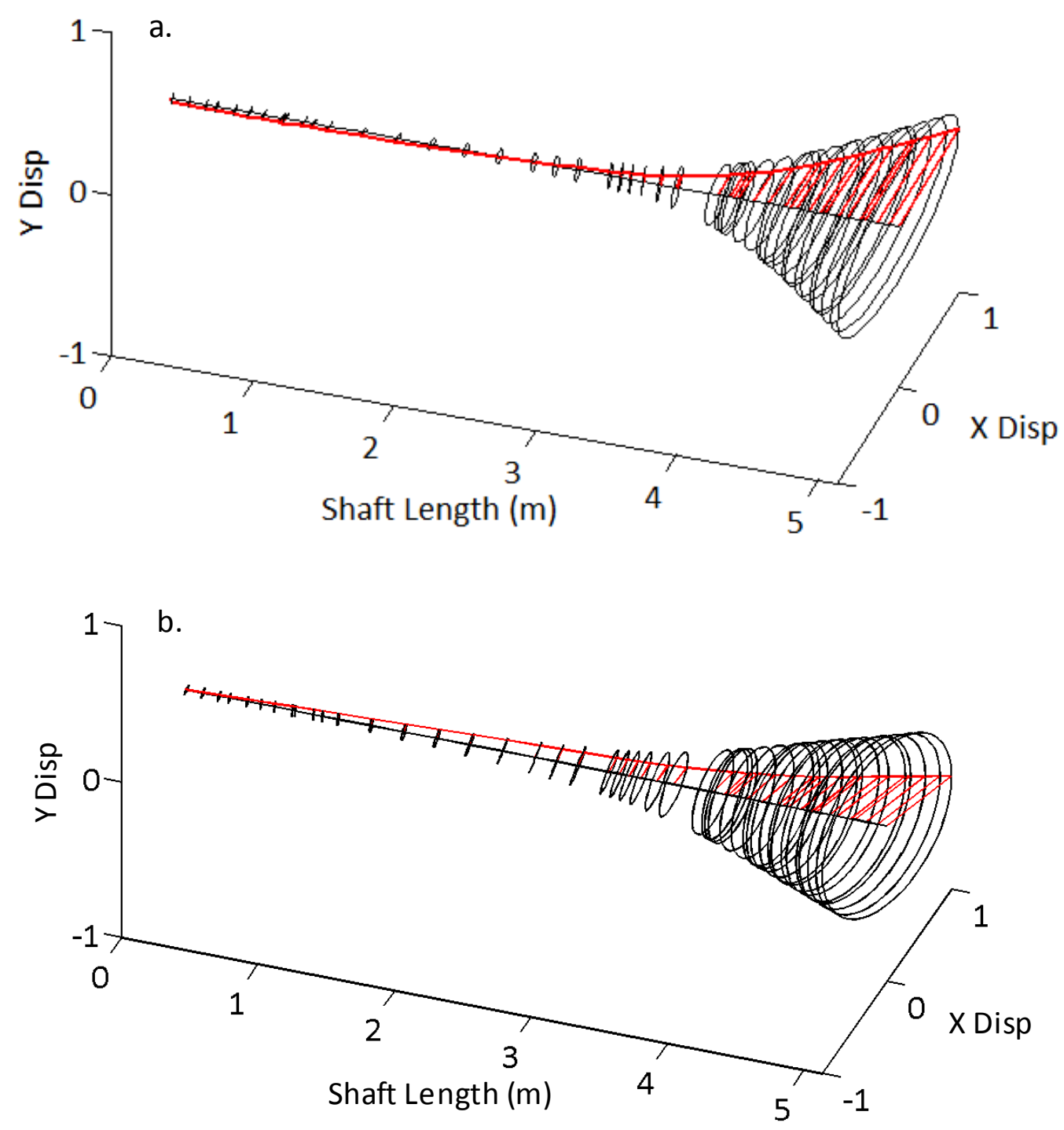

Figure 3.18. Mode 5 of the generator and low-speed pinion for the a. GEL and b. ISO fluid cases.

Mode 11, the first bending mode of the turbine, was studied further for the unbalance response and stability margin as a function of cross-coupled stiffness applied at the center of the shaft since this mode is more likely to be excited due to aerodynamic crosscoupling of the turbine blades or seal effects. Figure 3.19 shows the unbalance response of this mode for the three fluid cases, again showing very little effect of the fluid 
properties on the peak displacement of the shaft with a maxi-mum difference between the ISO and GEL of only a few micrometers. The stability margin of the first turbine mode (Figure 3.20) shows little difference between the ISO and POE cases, though the GEL case results in a decrease in the stability margin of about $15 \%$ due to the increased direct stiffness and reduced effective damping predicted for all speed cases. Overall, the turbine-generator system analysis shows that changing the bearing lubricants from petroleum-based oils to synthetics will not affect the rotordynamic performance in a significant manner, though at many speeds reducing the viscosity via the addition of $\mathrm{CO}_{2}$ may result in lower stability margins while passing through critical speeds during startup and shutdown. One way to avoid these undesirable effects while increasing bearing efficiency and reducing operating temperatures would be to perform startup and shut down operations with pure POE, then adding $\mathrm{CO}_{2}$ to the lubricant only while at operating speed. Similar to the compressor, however, this transition between steady-state conditions at operating speed would need to be studied using a transient analysis. The future development of a multi-rotor, transient rotordynamic code should assist in this analysis. Considering the effects of the high-pressure seals required for GEL lubrication should also be considered in future work. It is possible that their contributions to machine stability may be comparable to many current turbomachinery designs for high-pressure seals such as barrier seals, mechanical seals, bushing seals, etc., which are commonly employed to separate high-pressure end stages from low pressure bearings [103]. 


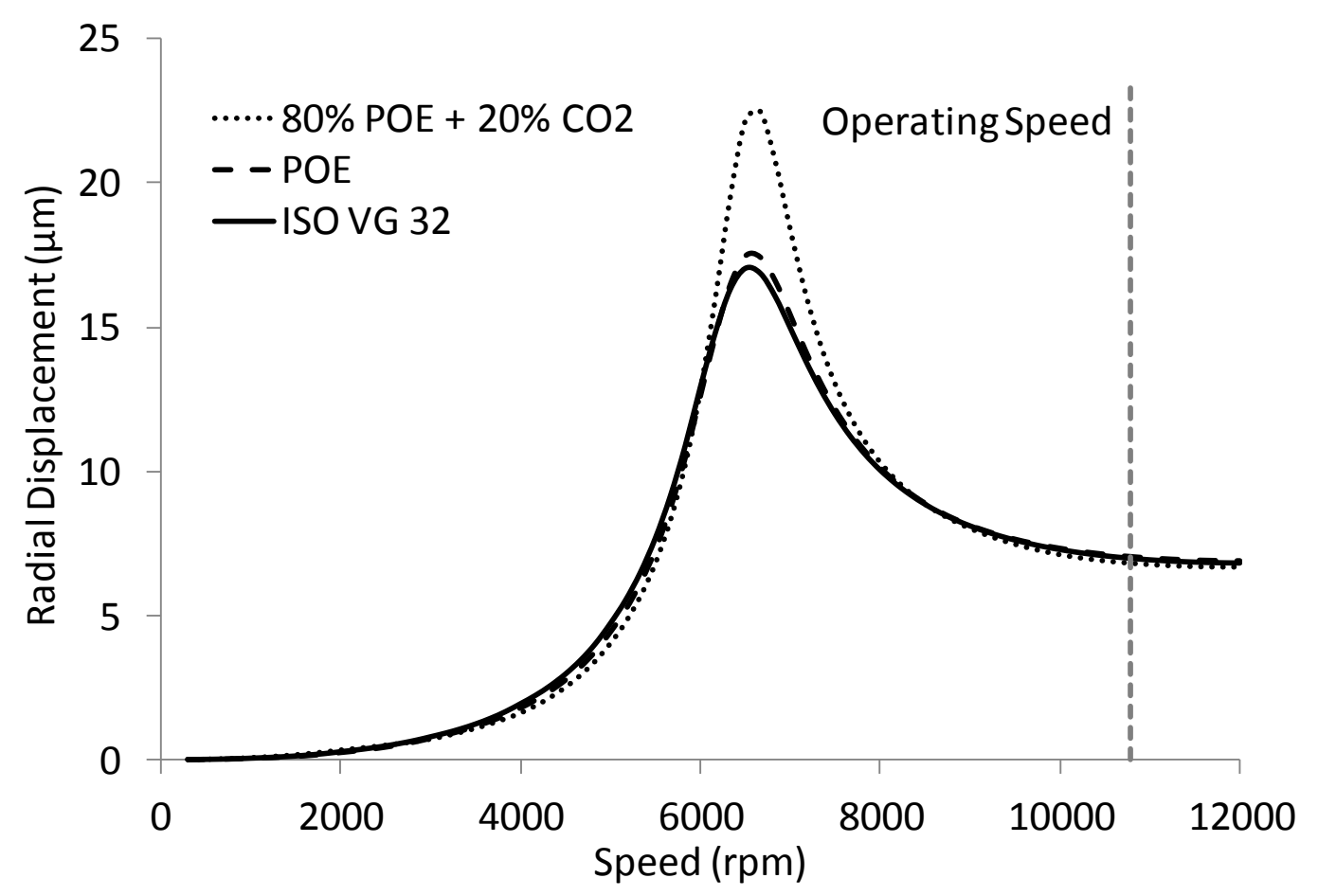

Figure 3.19. Unbalance response of the turbine first bending mode.

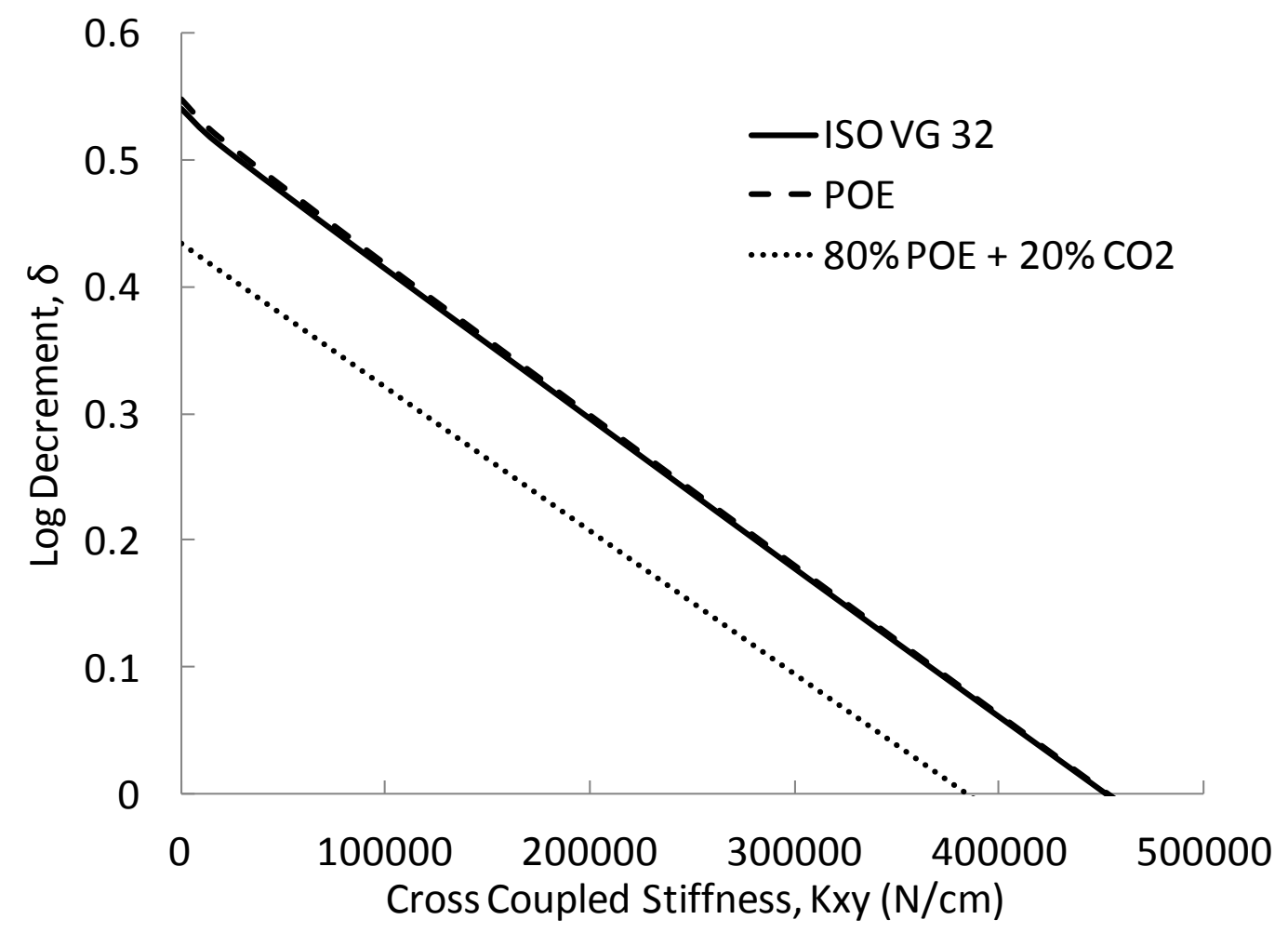

Figure 3.20. Steam turbine stability margin. 


\subsection{Conclusions}

The effects of GEL lubrication on turbomachinery bearing performance and rotordynamic stability were evaluated via two case studies. TEHD and finite element rotor models were employed to investigate these effects on a compressor and a steam turbine-generator system. The results for the centrifugal compressor showed favorable machine behavior in the GEL lubrication case, with significant reductions in bearing power loss and operating temperature expected along with an increase in the stability margin of the first bending mode. Transient rotordynamic results indicated that a relatively smooth transition between different sets of fluid properties is likely, with bearing coefficients being predictably controlled via lubricant viscosity. The turbinegenerator system showed bearing efficiency increases in the low speed bearings while the high speed bearings saw limited gains or even reductions in efficiency due to the onset of turbulent flow at higher operating speeds in the GEL case. Significant reductions in operating temperature in the high speed pinion bearings, along with a GEL base fluid with a slightly higher viscosity than the ISO VG 46, resulted in increased levels of power loss, significantly thicker lubricant films, and lower eccentricity ratios at higher operating speeds. The use of GELs also reduced the stability margins for many of the modes predicted for the turbine-generator system, suggesting that the overall effects of GELs on bearing coefficients and machine rotordynamics are machine-specific and dependent on the design of the bearing-rotor system. A few general trends have emerged on the effects of GEL lubrication on bearing coefficients in the analytical work performed to date including reduced direct stiffness and damping coefficients, particularly at higher speeds

and lower loads, but exceptions to these trends have also been found. In addition to 
effects on bearing coefficients and machine stability, other factors may also limit the amount of $\mathrm{CO}_{2}$ that could be added to the lubricant including film thickness/load capacity and the pressures required to achieve higher levels of dissolved $\mathrm{CO}_{2}$ in the fluid, though these factors are again dependent on machine design and would warrant a more parametric analysis of $\mathrm{CO}_{2}$ content for the machine being considered for GEL operation. The operational flexibility associated with the use of GELs could, however, prove to be most useful in machines where rotordynamic predictions favor the use of pure oil, allowing the user to add $\mathrm{CO}_{2}$ to the mixture after reaching operating speed to increase machine efficiency and increase bearing longevity through reduced operating temperatures. Considering the effects of bearing seals under GEL lubrication conditions should also be considered. Ongoing efforts to investigate the performance of seals with GELs should provide additional information on this subject. Overall, the potential for GELs to improve bearing efficiency, increase longevity, and provide a degree of rotordynamic control over high speed machines is encouraging and warrants further study. 


\section{Chapter 4}

\section{Nonlinear Analysis of Rub Impact in a Three-}

\section{Disk Rotor and Correction via Bearing and}

\section{Lubricant Adjustment}

\subsection{Introduction}

Rotor-stator rubbing is a significant concern among turbomachinery designers and operators. This detrimental behavior can result in machine damage ranging from increased wear and lower efficiency to catastrophic failure of the machine [9]. This contact can also significantly affect the rotordynamic performance of the machine by altering natural frequencies and mode stabilities while producing nonlinear vibration and nonperiodic, chaotic rotor motion. Radial rubbing in high-speed machines can be caused by a number of factors including excessive radial loading, rotor imbalance, misalignment, thermal growth, rotor bow, and excessive vibration. While solutions are dependent on the cause, imbalance and vibration-induced rubs can be addressed with field balancing of the 
rotor or magnetic bearing systems that control rotor vibration, approaches that typically require significant financial investment and machine downtime to implement.

The nonlinear behavior of rub impact in rotordynamic systems has been studied in various ways. Muszynska and Goldman [104] performed an analytical and experimental characterization of dynamic behavior in a rotor-rolling element bearing system caused by rubbing and a loose bearing pedestal. Their results demonstrated nonperiodic, chaotic vibration that could be reduced with increased system damping. Huang et al. [105] investigated analytically the dynamic behavior of a hydraulic generator unit under a misalignment and rub impact condition, finding that reducing the mass eccentricity and increasing bearing stiffness could reduce vibration and even restore periodic motion by increasing the natural frequencies of the system away from the operating speed. Sinha [106] studied the transient behavior of blade rubbing by modeling the blade as a Timoshenko beam with periodic pulse loading due to rubbing of the blade tip with the outer casing. Results included information on blade deformation and non-linear vibration, though system damping was neglected in this study. Cao et al. [107] studied the effects of speed, blade tip gap, and elastic coefficient on the nonlinear vibration of an aero engine rotor blade modeled as a continuous cantilever in a centrifugal force field. It was found that speed and blade tip gap had a significant effect on the vibration characteristics. Khanlo et al. [108] demonstrated the importance of Coriolis and centrifugal effects in the analytical prediction of dynamic behavior for a 1-disk flexible rotor under a rub impact condition. A more recent study [109] expanded their previous work to include the effects of bearing coefficients wherein they gradually increased direct stiffness, direct damping, cross-coupled stiffness, and cross-coupled damping individually to see the effect on the 
rub impact dynamics. It was found that for this rotor increasing the direct coefficients improved and in some cases even removed nonperiodic motion, whereas increasing the cross-coupled coefficients aggravated the chaotic behavior under some operating conditions, though real bearing designs - and their subsequent effects on bearing coefficients - were not considered. Wang et al. [110] modeled a Jefcott rotor supported by oil film bearings and concluded that by increasing the damping ratio that rub-induced nonlinear behavior could be improved or removed. While many of these studies have shown that bearing stiffness and damping coefficients could play a significant role in rub impact mitigation, none of them tied these specific bearing characteristics to realistic bearing designs that might be considered in a real machine troubleshooting scenario.

A number of bearing system design parameters can be considered in attempting to improve the dynamic response of a rotor. Barrett et al. [14] calculated optimum effective damping values for rotor stability and unbalance response by relating damping to stiffness ratio via bearing clearance and rigid bearing critical speed. Nicholas and Kirk $[15,16]$ discussed tilting pad journal bearing design for optimum rotordynamic performance, reporting that lower preloads, reduced bearing clearances, and increased L/D ratios typically increase damping. Load-between-pad bearing configurations were also found to reduce unbalance response. Lightly loaded bearings, however, were discussed as benefiting from preload as extremely light loads can result in destabilizing, negative bearing stiffness. Bhat et al. [17] optimized individual bearing design parameters for minimum unbalance response and found that increased bearing diameters, lower bearing clearances, higher lubricant viscosities, and increased L/D ratios reduced the unbalance response of a single-disk rotor. Untaroiu and Untaroiu [18] successfully 
developed a tilting pad bearing design optimization scheme by combining heuristic optimization algorithms with bearing and rotor finite element models to optimize bearing designs for efficiency, stability, and unbalance response. While these studies all provide critical insight into the effects of individual bearing design parameters on rotordynamic performance, it remains to be seen how these parameters in combination could be modified to minimize rotor displacements in an effort to reduce or remove a rub impact.

Various solutions to vibration-induced rub impact in high speed machines have been studied and proposed. Zhang and Wang [111] investigated the effects of phase difference between disks on rotordynamic response, highlighting field balancing of the rotor as a possible solution in rub impact mitigation. Wang et al. [112] studied field balancing analytically for a steam turbine case study and found this method to be inadequate for the conditions studied. As an alternative solution, a test rig was then utilized to study the use of an active magnetic exciter to control the vibration of the rotor during rub impact conditions, showing that this technology could effectively reduce imbalance-induced vibration. Chang-Jian and Chen [113] analytically studied the effectiveness of micropolar fluid film bearings for improving the vibration characteristics of a 1-disk flexible rotor under a rub impact condition, though it was concluded that the use of these fluids would be ineffective for this particular case. A later study by the same authors [114] found that the use of porous bearings could improve the vibration behavior of a rotor under a rub impact condition when compared to non-porous bearings. Wang [115] discussed the design of spherical gas bearings in the context of rub impact operating conditions and found that some designs could effectively remove nonperiodic, unstable behavior for a 1disk flexible rotor. 
In addition to bearing design parameters, modifying lubricant properties also has the potential to improve rotor behavior under rub impact conditions. Gas-expanded lubricants (GELs), tunable mixtures of synthetic oil and dissolved carbon dioxide, have been proposed to provide rotordynamic control in turbomachinery [116]. By controlling the pressure in GEL-lubricated bearings, the properties of these fluids can be controlled in real time, allowing operators to optimize machine performance using this control. Results from this recent work showed that tuning the lubricant properties could affect the unbalance response by up to five microns. However, in order to achieve more significant reductions in the peak displacement of the shaft it is hypothesized that a combination of bearing parameter adjustments would be required.

In this study, the effect of rub impact on the dynamic response of a 3-disk rotor is evaluated and a number of bearing system parameters are studied in combination to determine how much the peak displacement can be affected in attempts to remove the rub impact from the system and restore normal, synchronous motion. The rotordynamic characteristics of the bearing-rotor system are identified using a TEHD model of tilting pad journal bearing performance and finite element models of the 3-disk rotor studied. Results will then be presented in the form of a rotor critical speed map, peak rotor displacements, bearing stiffness and damping coefficients, bifurcation diagrams, a spectrum analysis, and whirl orbit shapes to demonstrate the effect of varying bearing characteristics on the dynamic response of the rotor-stator system. 


\subsection{Modeling Framework}

The modeling approach used in this study consisted of a two-step process of analyzing bearing performance and its subsequent effects on rotordynamic response. The first step involved modeling the behavior of the bearings, considering a number of bearing design parameters as variables including radial clearance, preload, pad orientation, and lubricant. An established thermoelastohydrodynamic (TEHD) finite element model described by $\mathrm{He}$ [76] was used to perform this step of the analysis. Stiffness and damping coefficients were calculated and were then used as inputs, in the second step, to finite element models for determining the rotordynamic response of the machine considered, allowing for an analysis of the potential effects of different bearing designs on rotor displacement and nonlinear behavior during rub impact conditions.

\subsubsection{Thermoelastohydrodynamic Bearing Model}

Section 2.3.2 provides a detailed description of the TEHD model used for this part of the study. This analysis produced sets of 8 synchronously reduced stiffness and damping coefficients that were used as inputs for each case into the below described finite element models of the rotor. This allowed the model to be used to simulate the speed-dependent characteristics of the fluid film bearings and how they interact with the entire rotor structure. Tables 4.1 and 4.2 outline the bearing geometry and fluid property inputs to the TEHD analysis. 
Table 4.1. Bearing inputs to the TEHD model.

\begin{tabular}{lcc}
\hline \hline Parameter & Value & Unit \\
\hline Shaft diameter & 50 & $\mathrm{~mm}$ \\
Rotational speed & $0-15000$ & $\mathrm{rpm}$ \\
Bearing radial clearance & $25.4,50.8$ & $\mu \mathrm{m}$ \\
Pad axial length & 12.7 & $\mathrm{~mm}$ \\
Pad arc length & 78 & $\mathrm{deg}$ \\
No. of pads & 4 & \\
Load orientation & $\mathrm{LOP}, \mathrm{LBP}$ & \\
Bearing preload & $0.2,0.6$ & \\
Pivot offset & 0.5 & \\
Bearing specific load & 364.7 & $\mathrm{kPa}$ \\
Oil supply temperature & 40 & ${ }^{\circ} \mathrm{C}$ \\
Oil supply rate & 7.6 & $\mathrm{~L} / \mathrm{min}$ \\
\hline \hline
\end{tabular}

Table 4.2. Fluid property inputs to the TEHD model.

\begin{tabular}{lccc}
\hline \hline Parameter & POE 2938 & GEL & Unit \\
\hline Density & 945 & 945 & $\mathrm{~kg} / \mathrm{m}^{3}$ \\
Specific Heat & 2.30 & 2.19 & $\mathrm{~J} / \mathrm{g} \cdot \mathrm{K}$ \\
Thermal Conductivity & 0.164 & 0.164 & $\mathrm{~W} / \mathrm{m} \cdot \mathrm{K}$ \\
Viscosity $40^{\circ} \mathrm{C}$ & 19.0 & 10.5 & $\mathrm{mPa} \cdot \mathrm{s}$ \\
Viscosity $100^{\circ} \mathrm{C}$ & 4.4 & 3.7 & $\mathrm{mPa} \cdot \mathrm{s}$ \\
Supply Pressure & 0.2 & 5.8 & $\mathrm{MPa}$ \\
Supply Temperature & 40 & 40 & ${ }^{\circ} \mathrm{C}$ \\
\hline \hline
\end{tabular}




\subsubsection{Steady-State Finite Element Rotordynamic Model}

Upon processing the performance data from the TEHD bearing model, a finite element model was developed for the 3-disk rotor being studied (Figure 4.1). The rotor is $95.5 \mathrm{~cm}$ in total length with a bearing span of $63.5 \mathrm{~cm}$. The rotor diameter is $5 \mathrm{~cm}$ with the outer disks having a diameter of $12.3 \mathrm{~cm}$ and the center disk having a diameter of 27 $\mathrm{cm}$. The length is broken down into 25 Timoshenko beam elements in the finite element model, with the bearings being represented as complex impedances at nodes 6 and 23 . The speed range considered was 0 to $15,000 \mathrm{rpm}$.

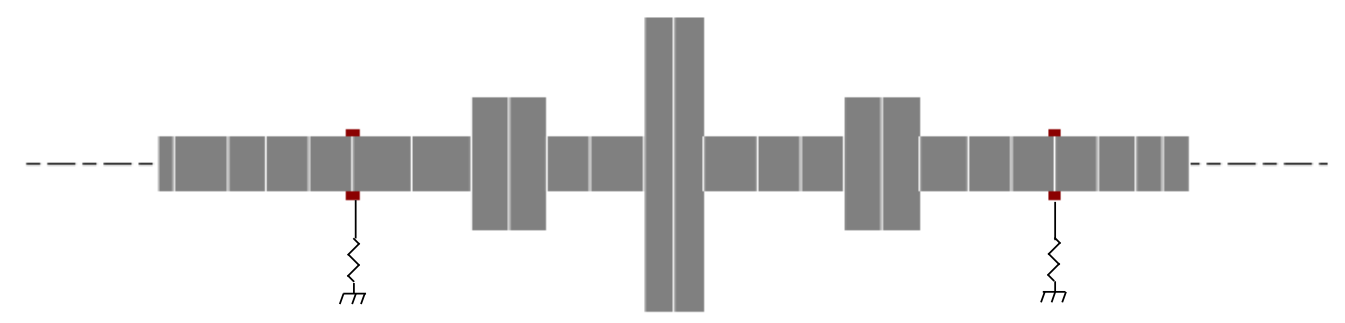

Figure 4.1. Finite element model of the 3-disk rotor.

The linearized flexible rotor model is represented by equation 3.4, containing terms representing the inertia, damping, gyroscopics, and stiffness matrices. The calculation of rotordynamic metrics including the locations of critical speeds and mode shapes allowed for a high-level analysis of the rotor vibration characteristics before using the below described nonlinear rotordynamic model to assess its behavior under a rub impact condition. Both forward and backward modes were considered in this analysis. 


\subsubsection{Nonlinear Finite Element Rotordynamic Model}

Similar to the steady state analysis, the nonlinear finite element model of the rotor is composed of 25 beam elements and considers shear and gyroscopic effects with bearing stiffness and damping forces applied at the bearing locations. Each node has 4 degrees of freedom, 2 translational and 2 rotational: $\mathrm{x}$ and $\mathrm{y}$ are translational freedoms in the $\mathrm{x}$ and $\mathrm{y}$ directions and $\theta_{\mathrm{x}}$ and $\theta_{\mathrm{y}}$ are the rotations of the cross section around the $\mathrm{x}$ and $\mathrm{y}$ axes.

The rub impact is assumed to take place at the center disk. Without considering thermal effects from friction, the rub impact forces are related to the radial displacement e with the following equation,

$$
\begin{array}{r}
\left\{\begin{array}{l}
F_{r x} \\
F_{r y}
\end{array}\right\}=-\frac{k_{r}\left(e-c_{r}\right)}{e}\left[\begin{array}{cc}
1 & -f_{c} \\
f_{c} & 1
\end{array}\right]\left\{\begin{array}{l}
x_{01} \\
y_{01}
\end{array}\right\} \quad\left(e \geq c_{r}\right) \\
F_{r x}=F_{r y}=0 \quad\left(e<c_{r}\right)
\end{array}
$$

where $\mathrm{e}=\sqrt{\mathrm{x}_{\mathrm{o} 1}^{2}+\mathrm{y}_{01}^{2}}$, and $\mathrm{x}_{\mathrm{ol}}$ and $\mathrm{y}_{\mathrm{o} 1}$ are the displacement components of the geometric center of the disk in the $\mathrm{x}$ and $\mathrm{y}$ directions. $\mathrm{F}_{\mathrm{rx}}$ and $\mathrm{F}_{\mathrm{ry}}$ denote the rub impact forces in the $\mathrm{x}$ and $\mathrm{y}$ directions. The clearance between the rotor and the stator $\mathrm{c}_{\mathrm{r}}$ is set at $80-120 \mu \mathrm{m}$, the radial stiffness of the stator $\mathrm{k}_{\mathrm{r}}$ is equal to $45 \mathrm{E} 6 \mathrm{~N} / \mathrm{m}$, and the friction coefficient between the rotor and stator $f_{c}$ is equal to 0.1 . The nonlinear equations of the system are integrated in the time domain using Newmark's implicit method. The model does not distinguish between the excitation of forward and backward modes, but characterizes the dynamic response of the system due to the forces applied to it. 


\subsection{Results and Discussion}

To evaluate the effects of a number of potentially important bearing parameters on the dynamic response of the rotor as it related to a rub impact condition, 16 cases were analyzed in the TEHD and rotordynamic models (Table 4.3). The parameters chosen for evaluation include the bearing lubricant, radial clearance, preload, and tilting pad orientation. The lubricants evaluated were a polyol ester (POE) synthetic oil and a GEL containing an $80 \%$ POE and a $20 \%$ carbon dioxide mass content. The bearing clearances and preloads chosen for evaluation represent the minimum and maximum values of ranges typically considered in bearing design. The two pad orientations chosen, load-onpad (LOP) and load-between-pad (LBP) configurations, also represent two commonly used tilting pad journal bearing designs.

Table 4.3. Bearing lubrication and design cases considered.

\begin{tabular}{ccccc}
\hline \hline Case & Lubricant & Radial Clearance & Preload & Pad Orientation \\
\hline 1 & POE & $50.8 \mu \mathrm{m}$ & 0.2 & LOP \\
2 & POE & $50.8 \mu \mathrm{m}$ & 0.2 & LBP \\
3 & POE & $50.8 \mu \mathrm{m}$ & 0.6 & LOP \\
4 & POE & $50.8 \mu \mathrm{m}$ & 0.6 & LBP \\
5 & POE & $25.4 \mu \mathrm{m}$ & 0.2 & LOP \\
6 & POE & $25.4 \mu \mathrm{m}$ & 0.2 & LBP \\
7 & POE & $25.4 \mu \mathrm{m}$ & 0.6 & LOP \\
8 & POE & $25.4 \mu \mathrm{m}$ & 0.6 & LBP \\
9 & GEL & $50.8 \mu \mathrm{m}$ & 0.2 & LOP \\
10 & GEL & $50.8 \mu \mathrm{m}$ & 0.2 & LBP \\
11 & GEL & $50.8 \mu \mathrm{m}$ & 0.6 & LOP \\
12 & GEL & $50.8 \mu \mathrm{m}$ & 0.6 & LBP \\
13 & GEL & $25.4 \mu \mathrm{m}$ & 0.2 & LOP \\
14 & GEL & $25.4 \mu \mathrm{m}$ & 0.2 & LBP \\
15 & GEL & $25.4 \mu \mathrm{m}$ & 0.6 & LOP \\
16 & GEL & $25.4 \mu \mathrm{m}$ & 0.6 & LBP \\
\hline \hline
\end{tabular}


To begin the analysis, a critical speed map of the system was developed (Figure 4.2). Two forward and two backward critical speeds were found to exist in the operating speed range considered. A mode shape analysis found that the first critical speeds were first rigid modes while the second critical speeds were conical modes. The sets of direct, vertical stiffness coefficients $\left(\mathrm{K}_{\mathrm{yy}}\right)$ superimposed over the lines denoting the critical speeds demonstrate the full range of bearing stiffness values calculated by the TEHD analysis, showing that only in cases of reduced bearing stiffness do the conical modes fall within the operating range considered for this rotor. The two groups of vertical stiffness values observed were found to be formed primarily due to differences in pad orientation and bearing clearance, with the lower values being calculated for a number of loadbetween-pad and larger bearing clearance cases.

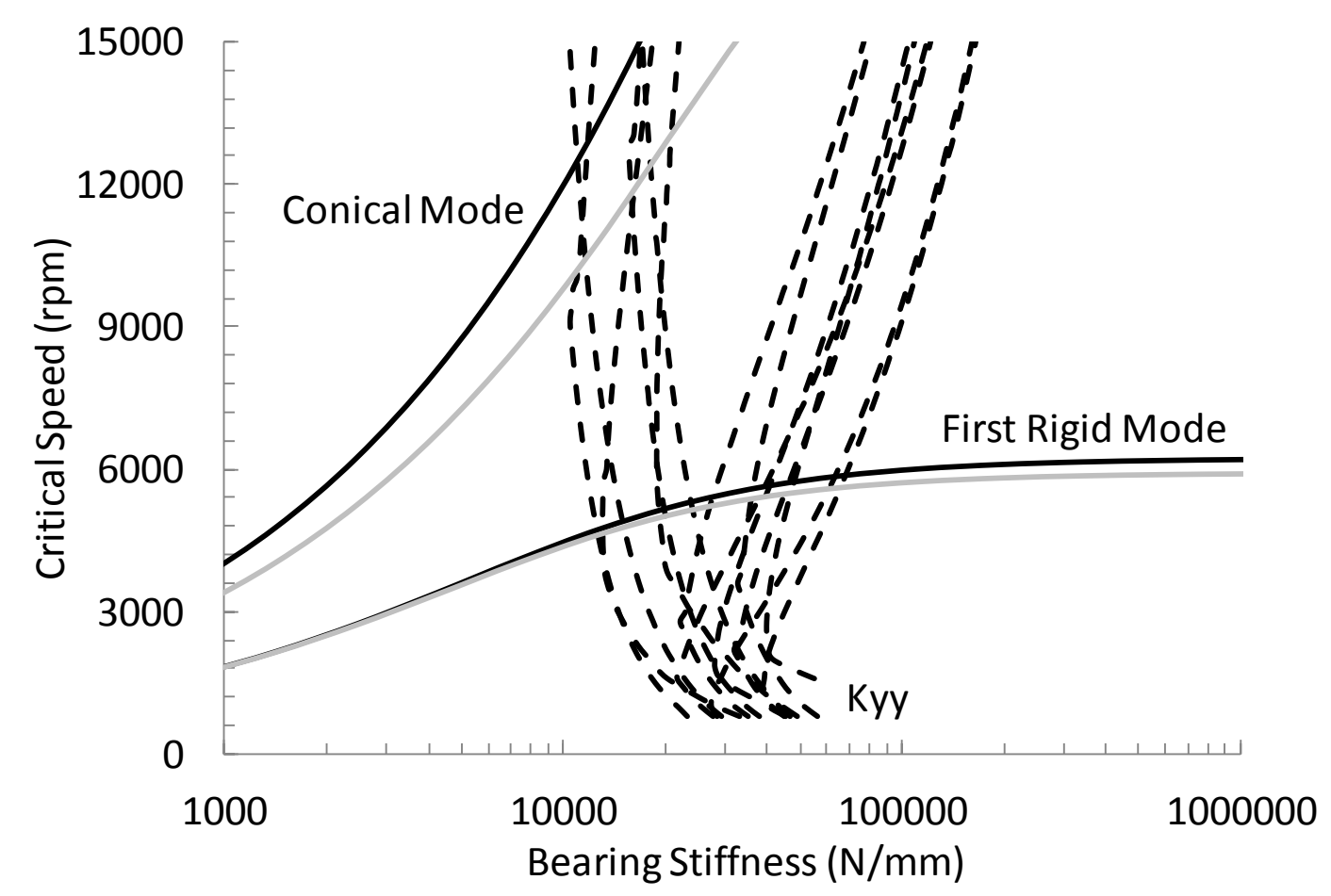

Figure 4.2. Critical speed map of the 3-disk rotor, showing both forward (black lines) and backward (grey lines) modes. 
Upon characterizing the overall rotordynamic nature of the system, unbalance forces were applied at the 3 disk locations to excite the rotor in a manner that would produce a rub impact condition. The nonlinear rotordynamic analysis was then performed for the 16 cases studied to evaluate the effect of the bearing characteristics considered on the dynamic response of the system. Of the 16 cases analyzed, 14 cases converged properly and are summarized below. Cases 11 and 12 did not converge for many speed cases in the TEHD analysis and produced negative direct damping values that also disallowed the rotordynamic code from converging. This was due perhaps to the unlikely combination of a low viscosity lubricant, large bearing clearance, and high bearing preload. Hence, the results of these cases have been excluded.

The center disk radial displacements of the first set of cases considered are shown in Figure 4.3. The displacements rapidly increase around 4,500-6000 rpm as the rotor passes through the first critical speed and remain relatively high with unbalance forces persisting as the system approaches the second mode. The preload of 0.2 and load-between-pad orientation were constant for these cases, highlighting the relative importance of the two variables in this comparison, the lubricant and the bearing clearance. The bearing clearance was found to have a more significant impact on the peak rotor displacements, with the larger bearing clearance of $50.8 \mu \mathrm{m}$ producing lower radial displacements up to approximately $11,000 \mathrm{rpm}$. In these cases the rub impact is effectively removed until higher speeds are reached. The lubricant had a smaller effect on the displacements, with the POE producing lower values in the larger bearing clearance cases and the GEL producing lower values in the lower bearing clearance cases. The small impact of the fluid properties is consistent with the results of Weaver et al. [116]. The speed at which 
the initial peak displacements were reached was also higher in cases 6 and 14, found to occur due to increased bearing stiffness in the lower bearing clearance cases (Figure 4.4). These stiffness values as well as the higher levels of cross-coupled stiffness encountered in these cases also produced larger rotor displacements as effective damping was reduced.

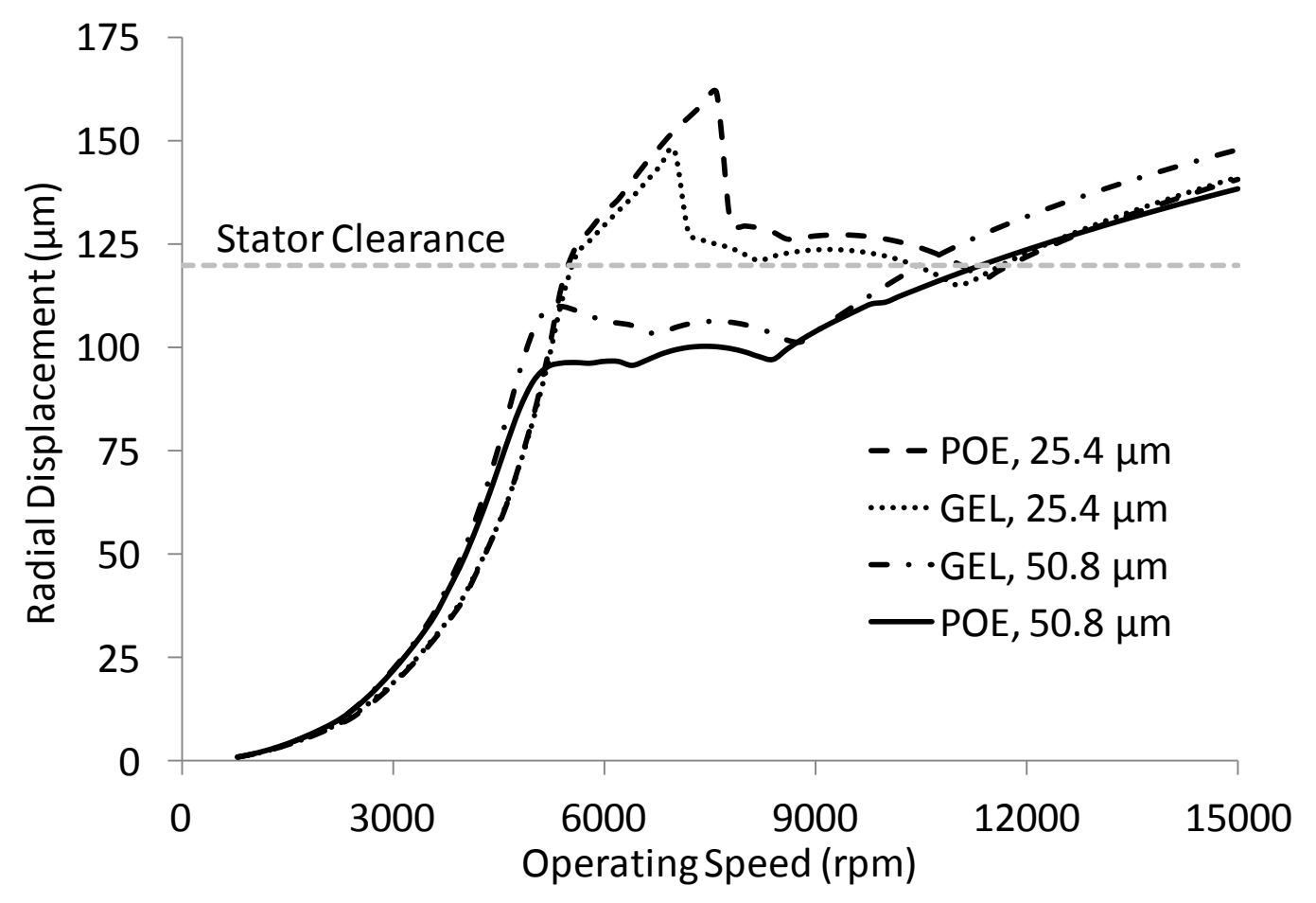

Figure 4.3. Center disk displacements for the 0.2 preload and load-between-pad bearing configurations. 

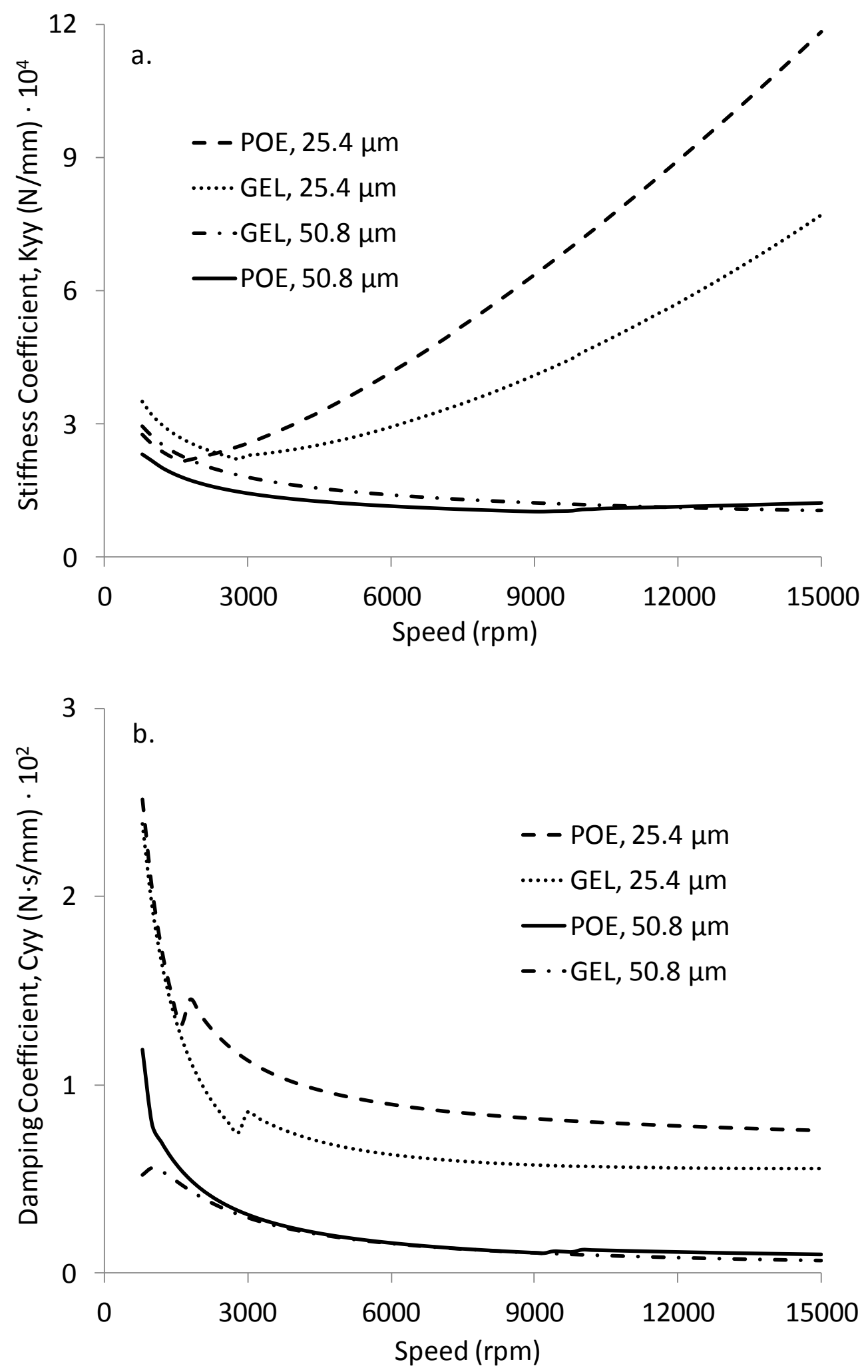

Figure 4.4. Dynamic stiffness (a) and damping (b) coefficients for cases 2, 6, 10, and 14 . 
Cases 2 and 14 were further analyzed for their dynamic behavior at a radial clearance of $80 \mu \mathrm{m}$ to highlight the different rotordynamic responses under mild-rub and severe-rub impact conditions. Figure 4.5 shows the $\mathrm{x}$-displacement bifurcation of the rotor motion for these sets of conditions, respectively, demonstrating abundant nonlinear behavior in the severe rub case. This behavior is also shown in the whirl orbit shapes at 9,000 rpm (Figure 4.6), with synchronous motion demonstrated in the mild-rub condition and chaotic motion found in the severe rubbing case. A spectrum analysis (Figure 4.7) reveals a doubling of the $1 \mathrm{X}$ vibration amplitude along with the addition of a significant $1.25 \mathrm{X}$ vibration under the severe rub impact condition. 

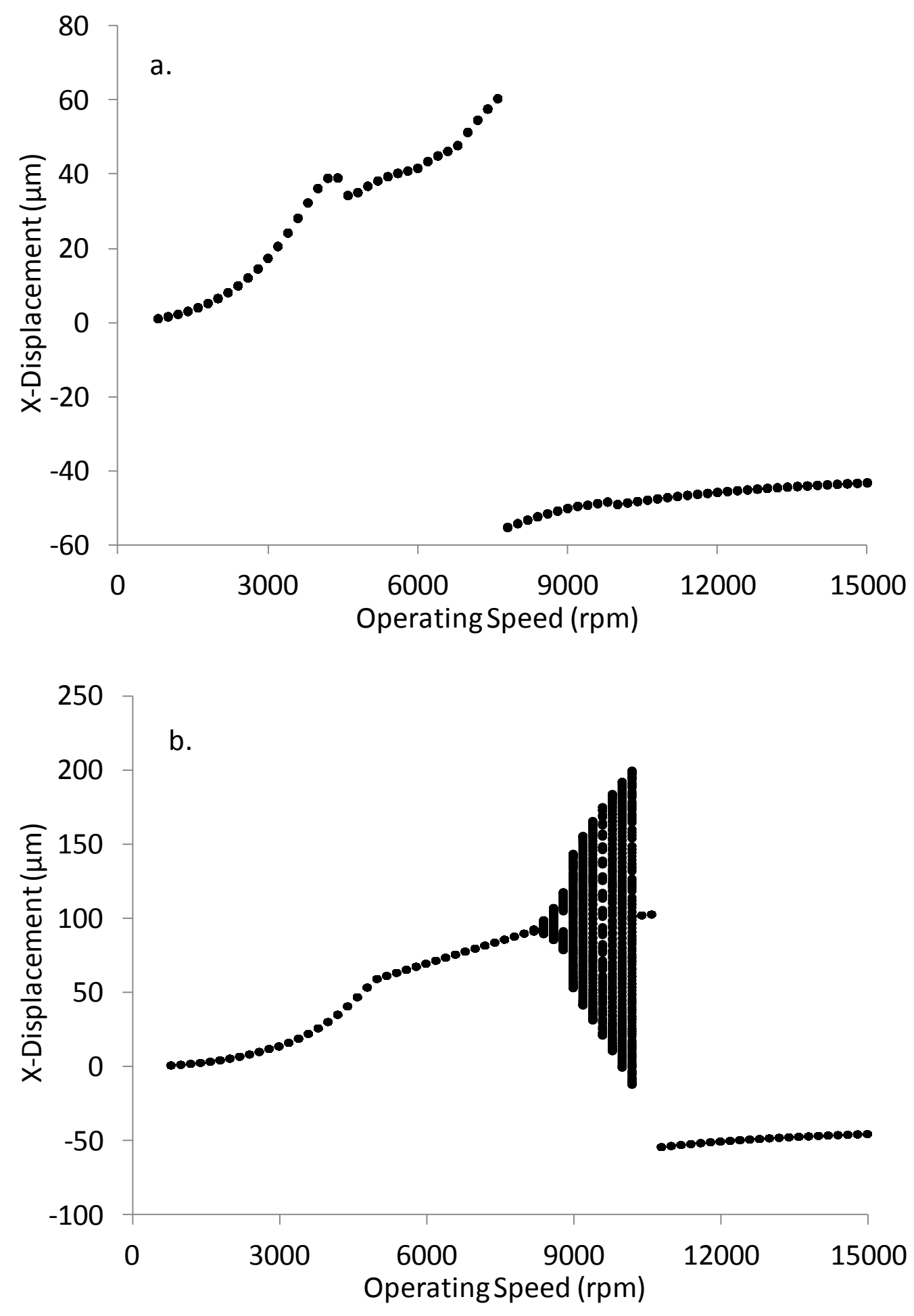

Figure 4.5. Bifurcation diagrams for cases 2 (a) and 14 (b). 

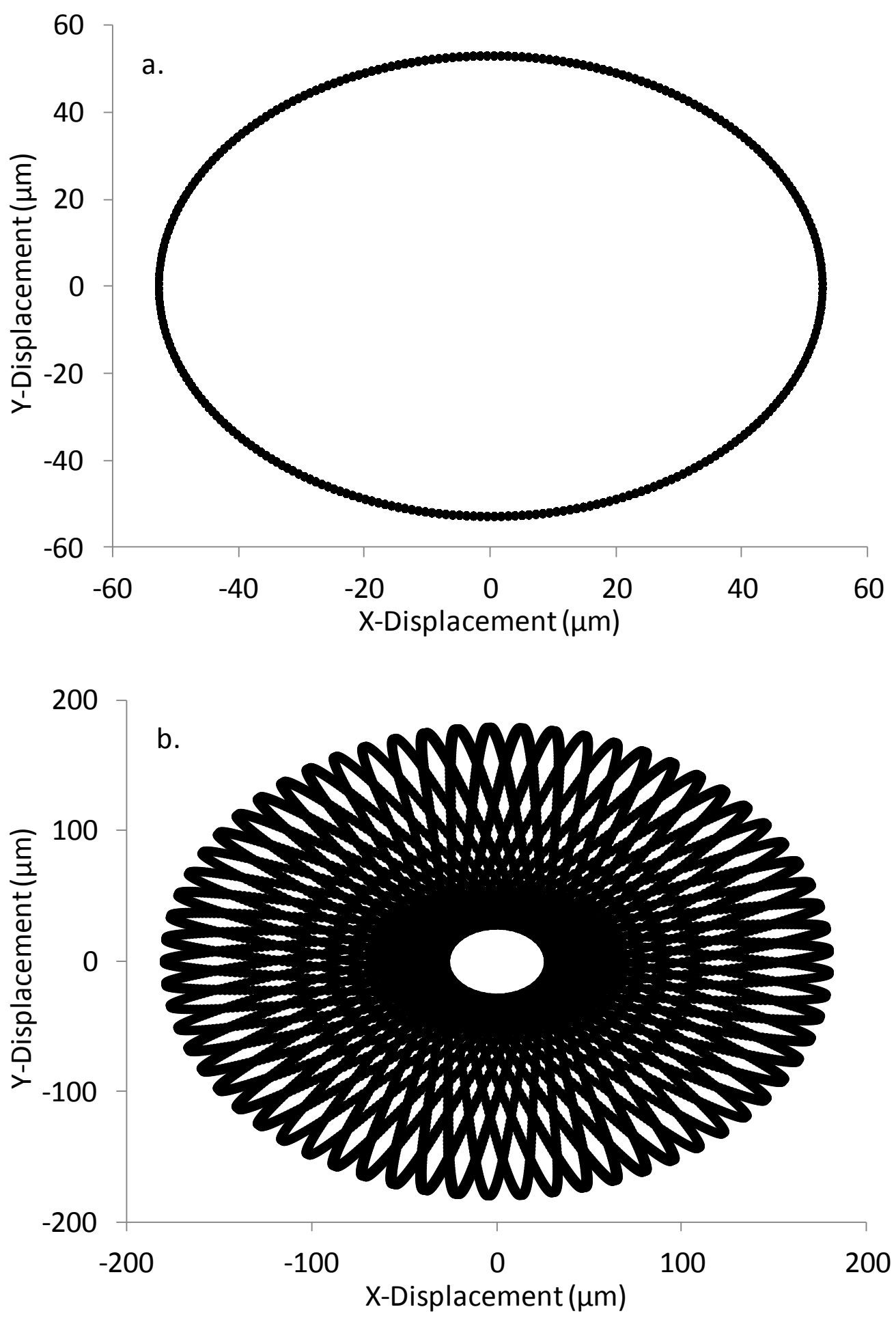

Figure 4.6. Shaft whirl orbits for cases 2 (a) and 14 (b). 

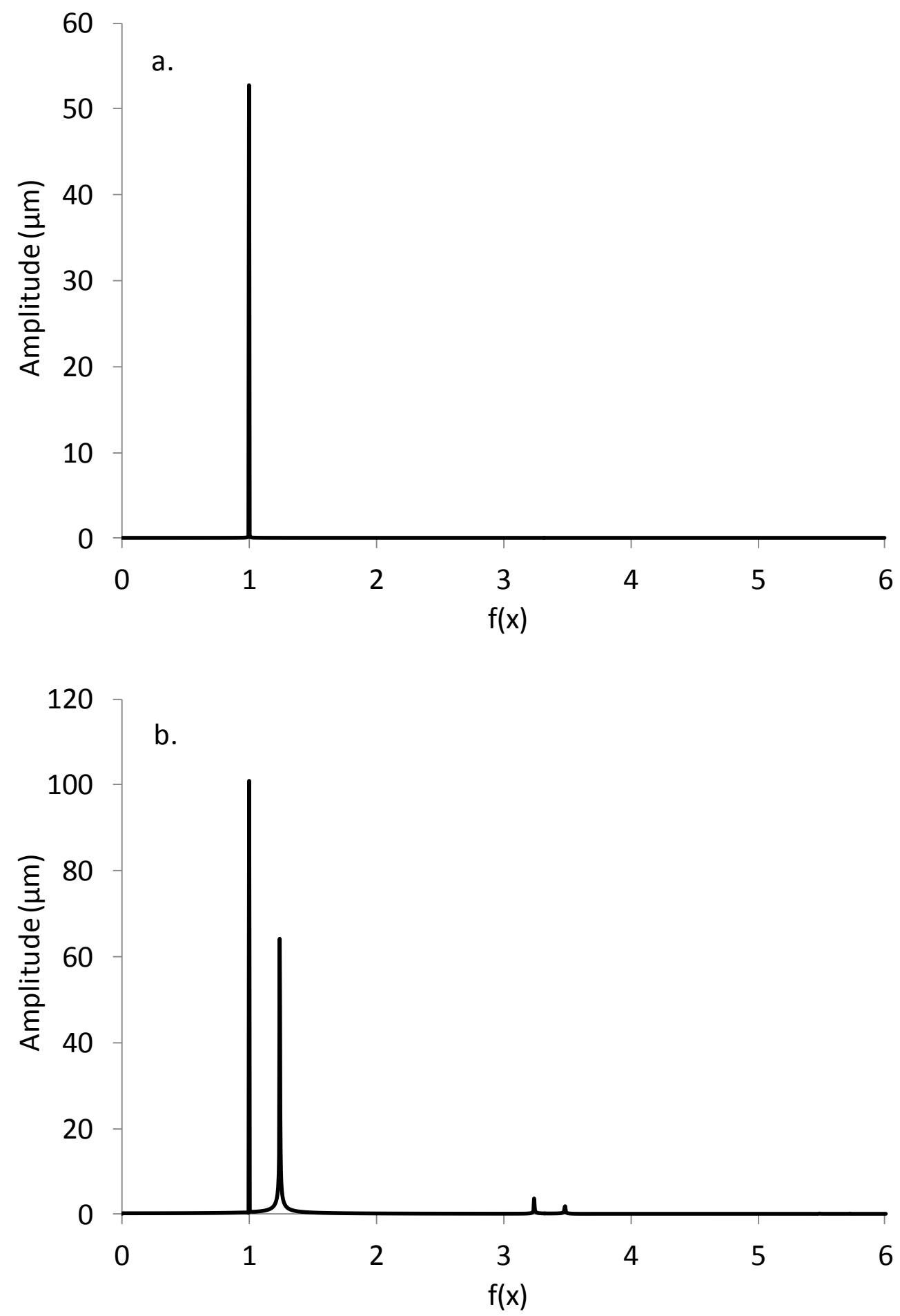

Figure 4.7. Spectrum analysis of cases 2 (a) and 14 (b).

A second set of bearing cases were analyzed for peak displacement, shown in Figure 4.8. Like the first set, the preload of 0.2 was constant for these cases, though a load-on- 
pad bearing configuration was implemented here. Unlike the first set, however, all of the cases compared reached a rub impact condition upon traversing the first mode at approximately 5,500-7,500 rpm, suggesting that load-between-pad bearing configurations are better suited for minimizing peak displacements. This is consistent with the results of Nicholas and Kirk [15, 16]. Case 1, utilizing a POE lubricant and a larger bearing clearance, produced the lowest peak displacements at the first mode but was comparable to the other cases at higher speeds. At the lower bearing clearance the GEL produced a slightly lower displacement. Cases 9 and 13 were fairly comparable, showing little effect of the bearing clearance in the GEL lubricant cases. These results were verified by the trends observed in the bearing stiffness and damping coefficients (Figure 4.9). While damping in these cases was comparable to the first set of cases analyzed, bearing stiffness values were generally higher, resulting in the larger displacements observed. Similar to the first set of cases compared, higher levels of bearing stiffness were also associated with the lower bearing clearance cases. 


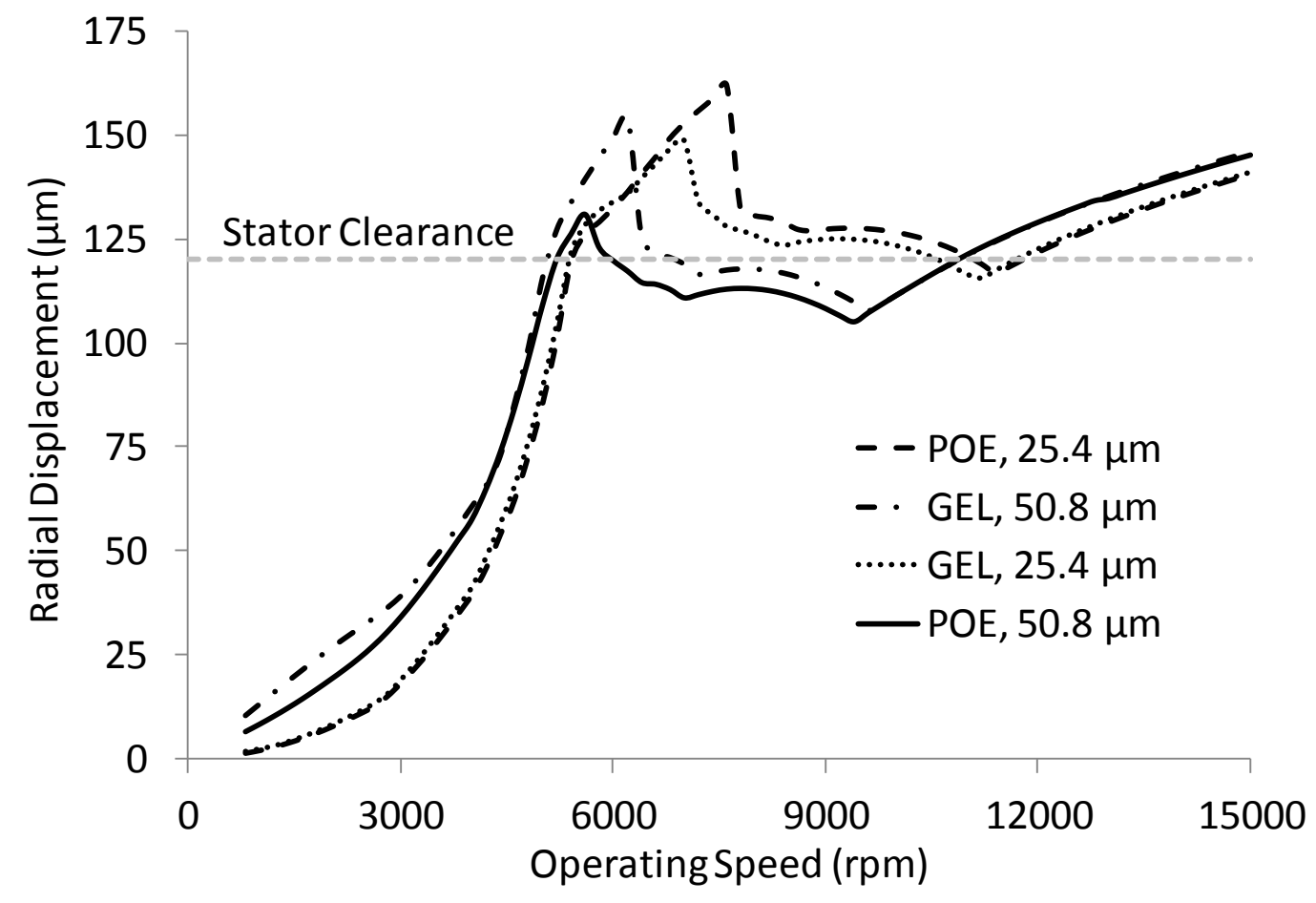

Figure 4.8. Center disk displacements for the 0.2 preload and load-on-pad bearing configurations. 

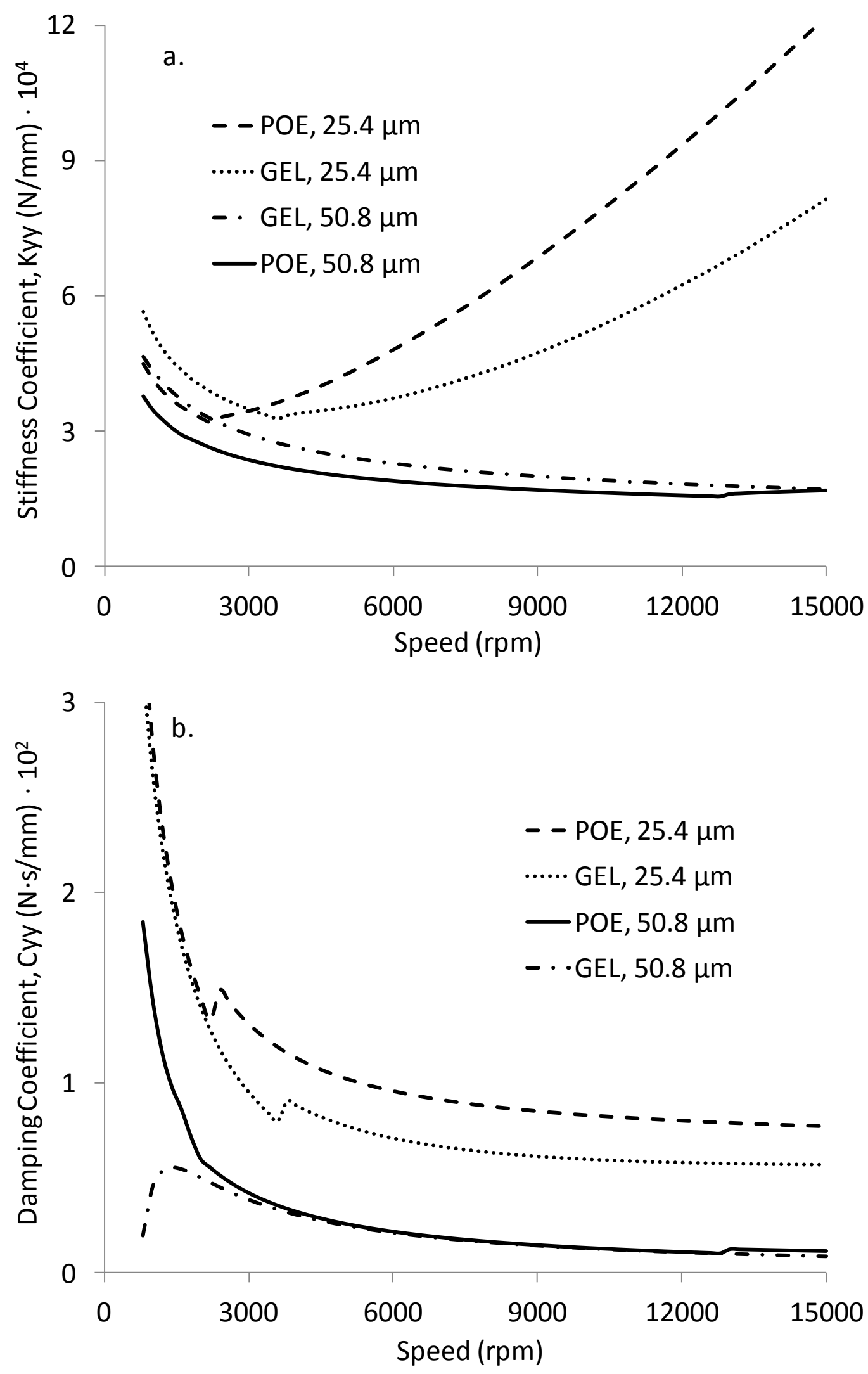

Figure 4.9. Dynamic stiffness (a) and damping (b) coefficients for cases 1, 5, 9, and 13 . 
A third set of bearing cases (Figure 4.10) allowed for a comparison of the other two variables for their effect on peak rotor displacements. In these cases the POE lubricant and $50.8 \mu \mathrm{m}$ bearing clearance were held constant while the preload and pad orientation were varied. Cases 2 and 4 produced the lowest peak displacements at the first mode, further suggesting that the load-between-pad configuration is the more ideal configuration. The effect of preload, though slightly smaller than pad orientation, was also significant with the 0.2 preload cases producing lower displacements that the 0.6 preload cases. This trend is also evident in the bearing stiffness and damping coefficients (Figure 4.11). Overall, stiffness and damping values were relatively low when compared to many of the other cases previously discussed, with case 2 benefitting the most from lower stiffness and higher damping values. The load-on-pad bearing configurations predictably produced greater vertical stiffness and damping values whereas the loadbetween-pad cases distributed stiffness and damping forces more evenly in the $\mathrm{x}$ and $\mathrm{y}$ directions, though the load-between-pad cases experienced greater overall levels of effective damping. 


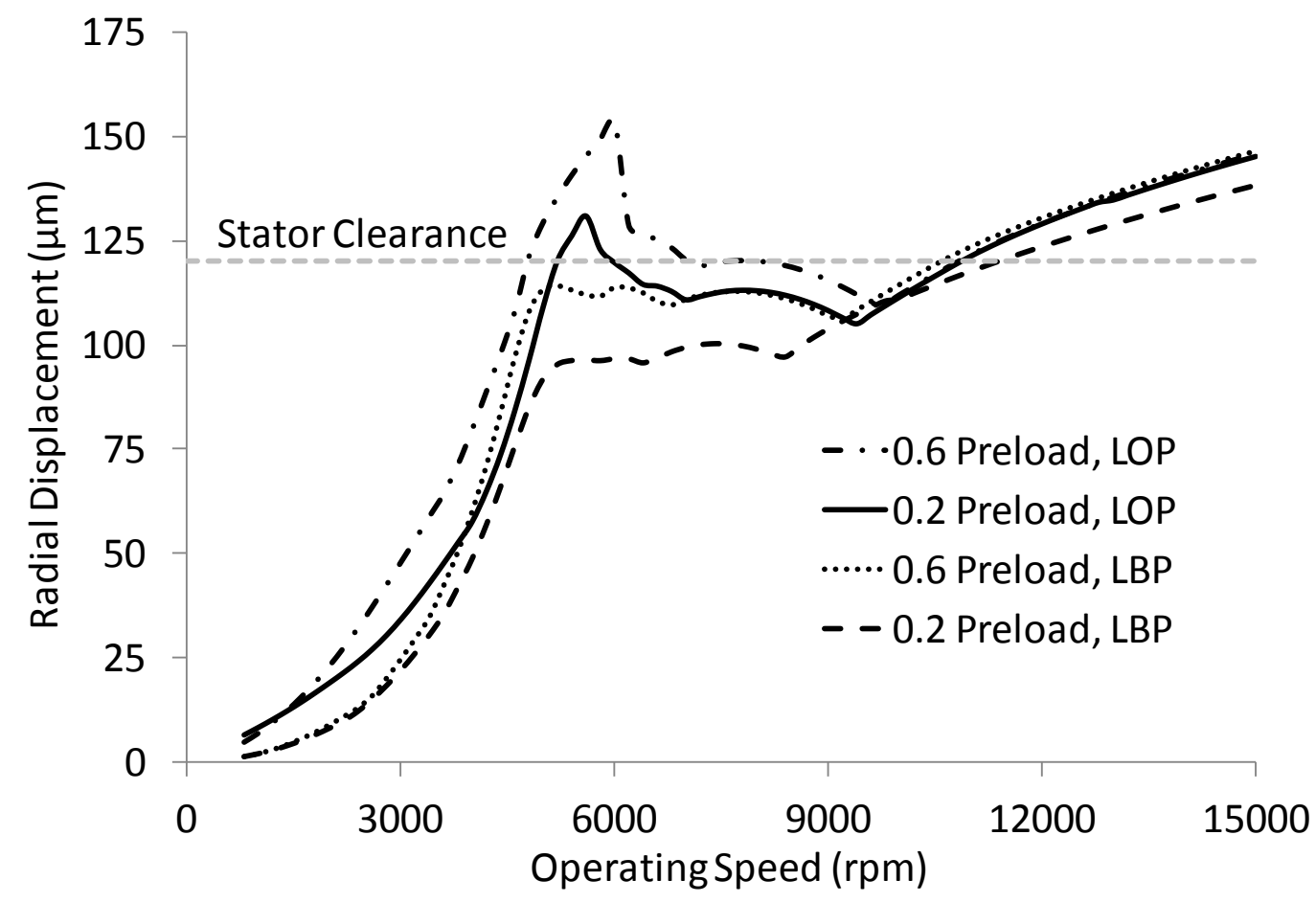

Figure 4.10. Center disk displacements for the POE lubricant and the $50.8 \mu \mathrm{m}$ bearing clearance. 

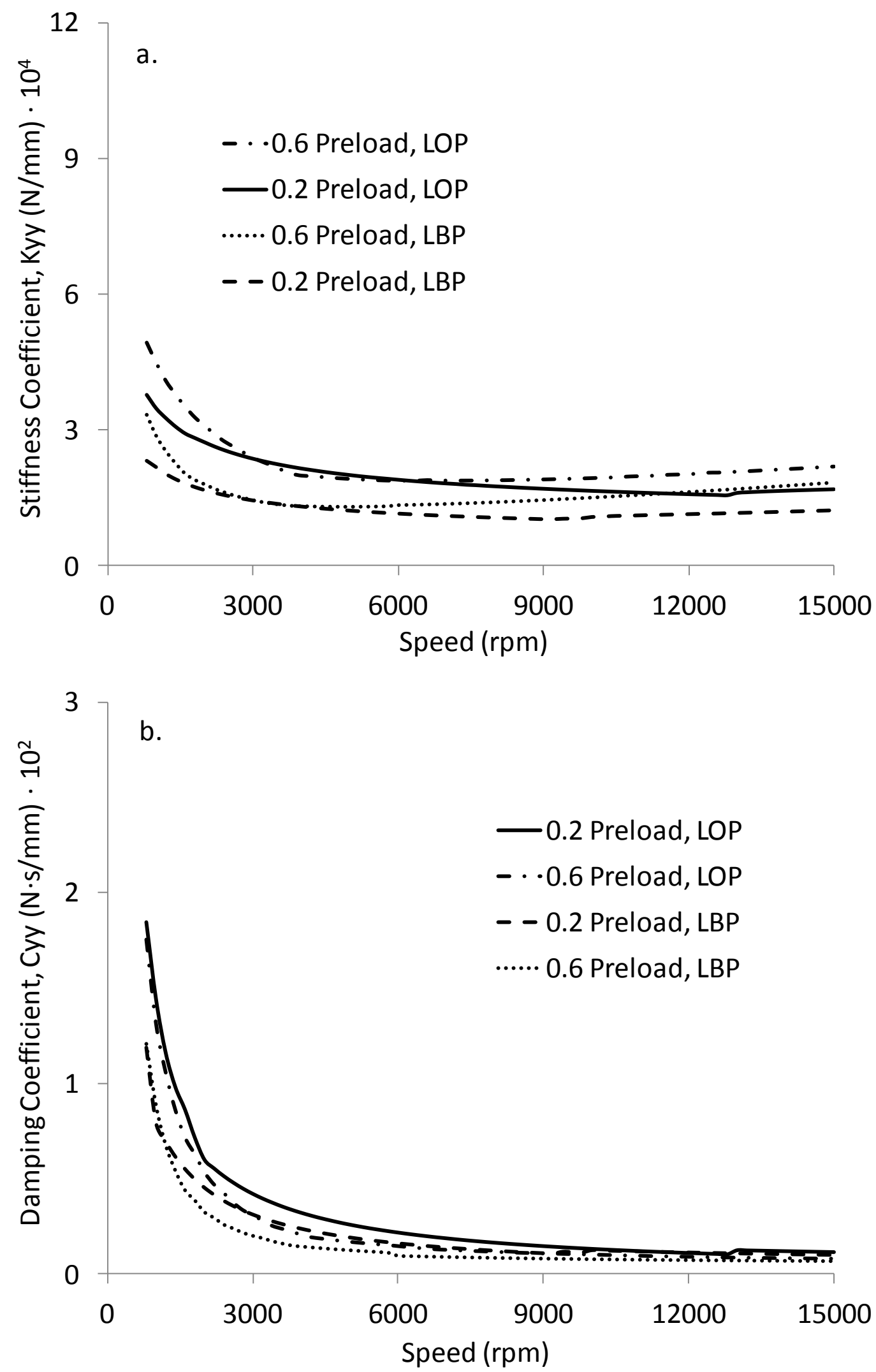

Figure 4.11. Dynamic stiffness (a) and damping (b) coefficients for cases 1, 2, 3, and 4 . 
To see if these trends held for the same lubricant but for a lower bearing clearance, cases 5, 6, 7, and 8 were analyzed for similar behavior (Figure 4.12). The strong influence of bearing clearance was evident in this comparison, with all four cases producing similarly large displacements. Cases 5 and 6, having the lower preload of 0.2 , again exhibited slightly lower displacements than the higher preload cases. The effect of bearing orientation was much less pronounced than in the previous comparison under a larger bearing clearance, with differences between load-on-pad and load-between-pad cases being on the order of a few microns. This behavior was confirmed by the stiffness and damping coefficient results (Figure 4.13). The reduced bearing clearance produced higher overall levels of bearing stiffness along with moderate levels of damping. The higher preload cases (7 and 8) produced larger stiffness and lower damping forces, resulting in the larger rotor displacements predicted.

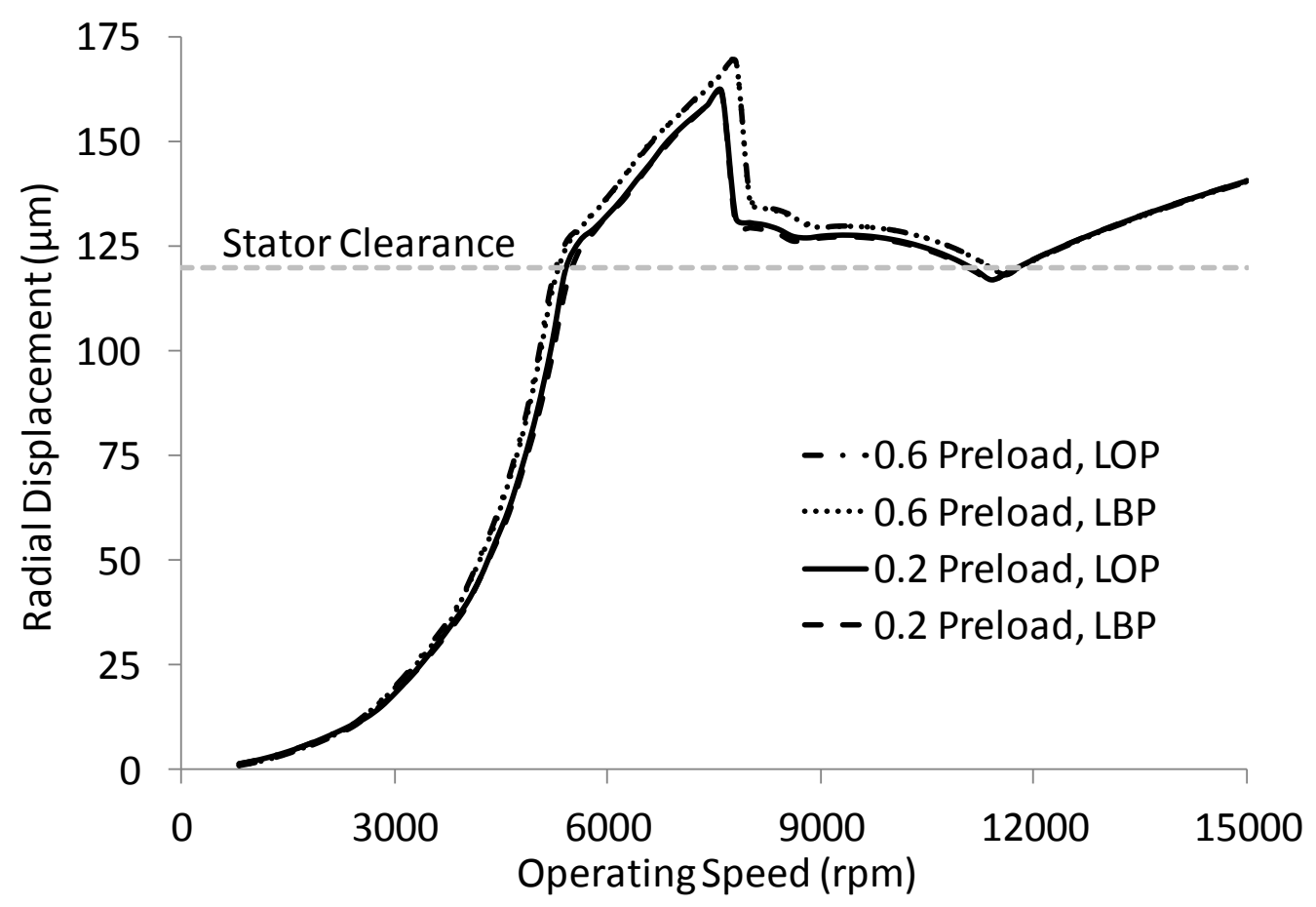

Figure 4.12. Center disk displacements for the POE lubricant and $25.4 \mu \mathrm{m}$ bearing clearance. 

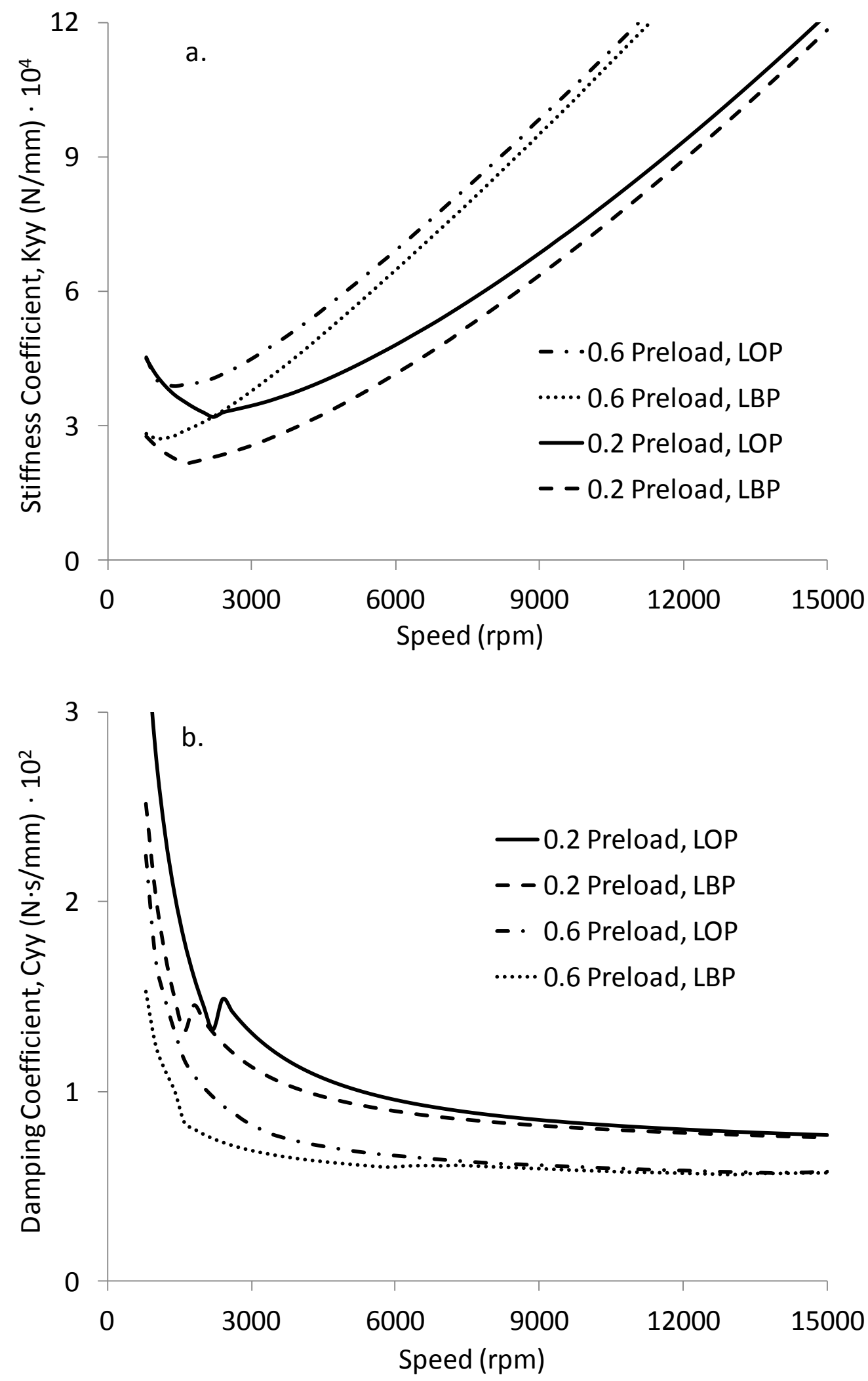

Figure 4.13. Dynamic stiffness (a) and damping (b) coefficients for cases 5, 6, 7, and 8 . 
The final set of cases compared for their performance were the GEL-lubricated bearings (Figure 4.14). The majority of GEL cases produced higher overall radial displacements during the first mode. Case 10 performed the best during this mode with its design including a larger bearing clearance, a lower preload, and a load-between-pad configuration. Cases 15 and 16, designed to have lower bearing clearances and higher preloads, produced the largest peak displacements. The bearing configuration again appeared to have a large impact on the system only in the higher bearing clearance cases. The bearing coefficient results verify this behavior (Figure 4.15), with case 10 having the lowest levels of bearing stiffness and cases 15 and 16 exhibiting the highest.

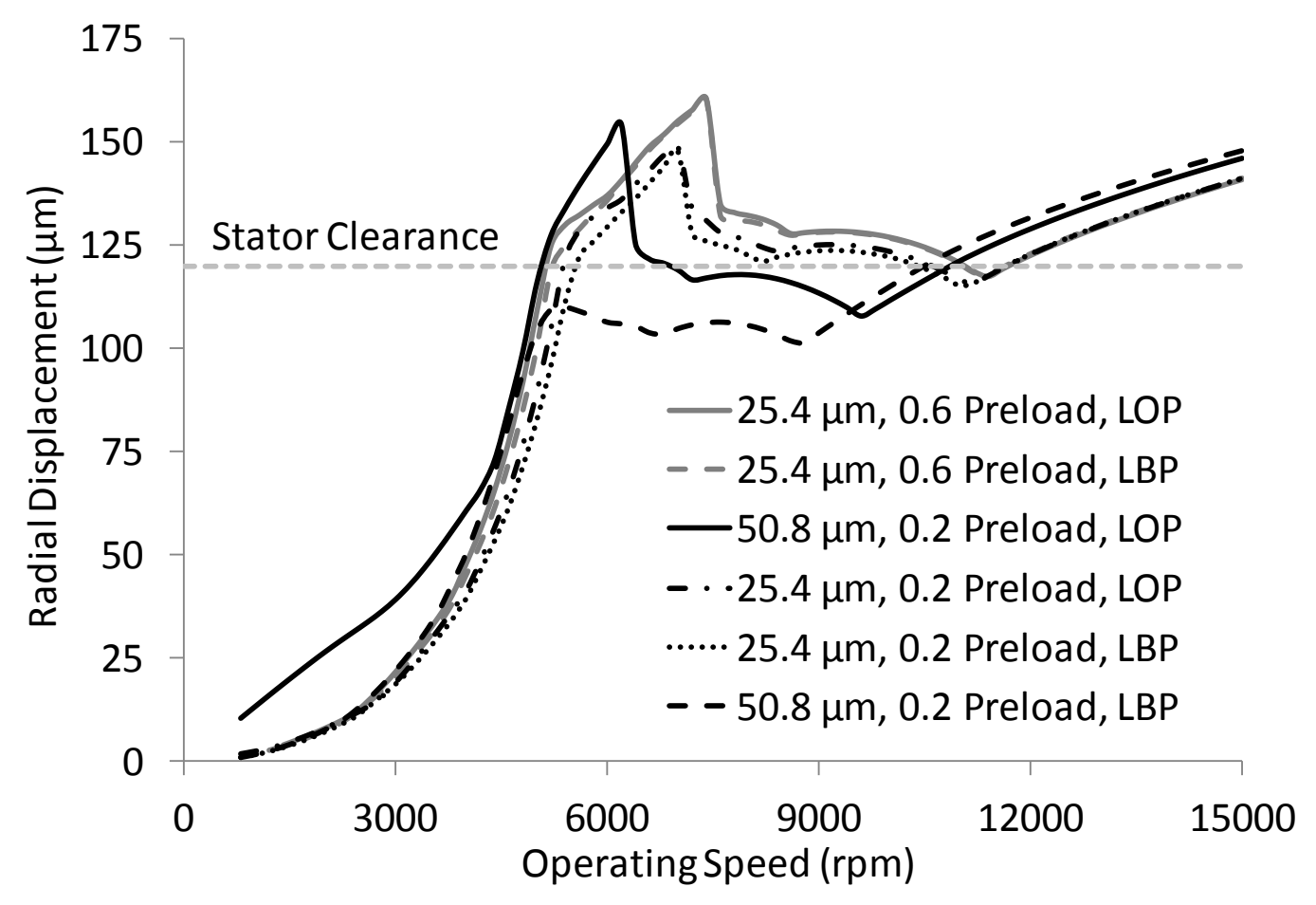

Figure 4.14. Center disk displacements for the GEL lubricant cases. 

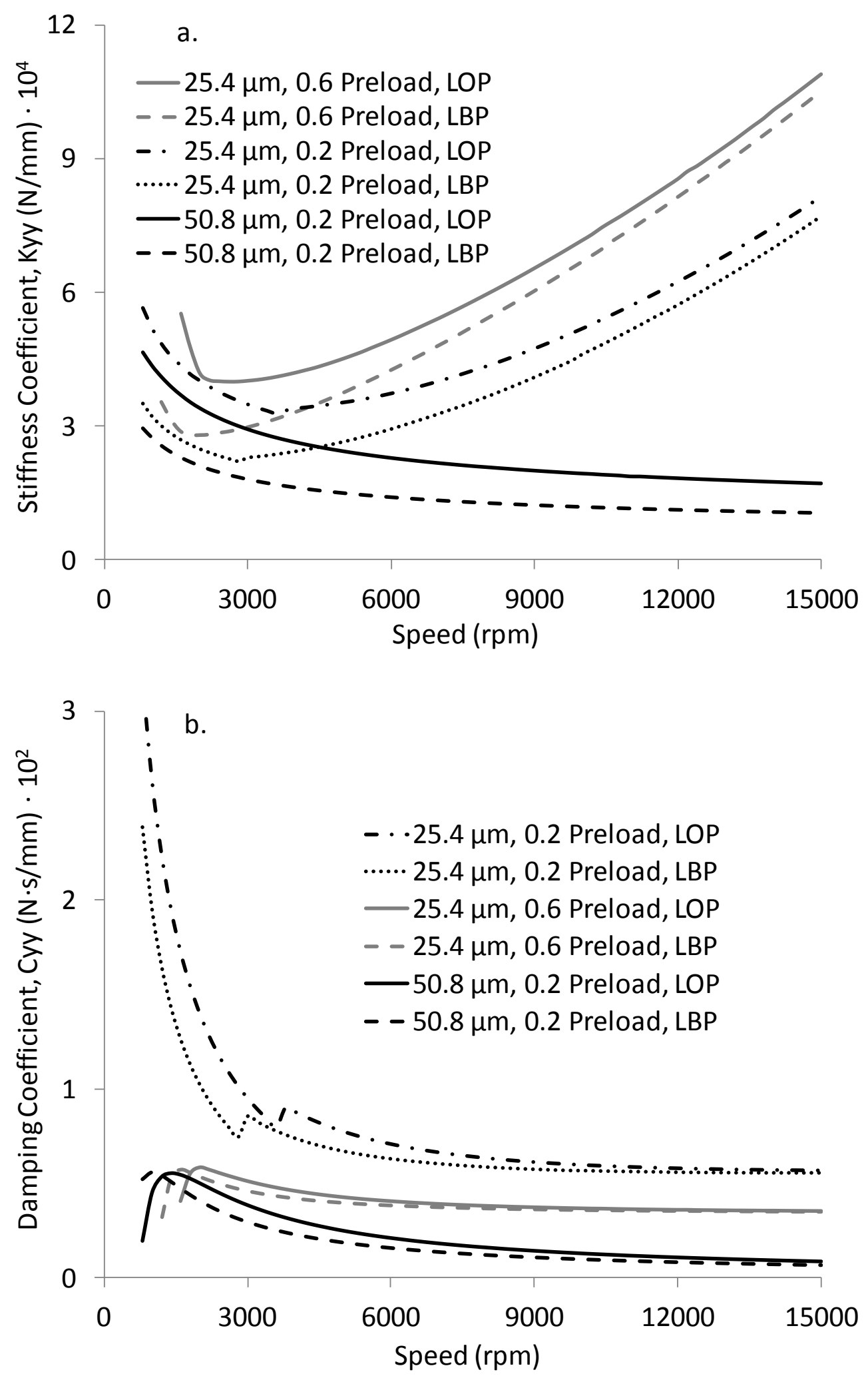

Figure 4.15. Dynamic stiffness (a) and damping (b) coefficients for cases 9, 10, 13, 14, 15 , and 16. 


\subsection{Conclusions}

A number of important bearing lubrication and design parameters were evaluated for their impact in improving the performance of a 3-disk rotor under a rub impact condition. TEHD and finite element models were employed to investigate these effects in terms of peak rotor displacements during machine operation as well as to highlight the differences in system behavior between the no-rub and rub impact scenarios. The results showed that large reductions in peak displacement were achievable when bearing design and lubrication parameters were optimized. While all cases predicted rub impacts at higher speeds for this heavily unbalanced rotor, the rub impacts encountered at the first critical speed were removed for cases in which optimal bearing conditions were utilized. The POE lubricant produced the lowest peak displacements for the larger bearing clearance cases while the GEL performed more ideally in some of the lower bearing clearance cases, showing that in many instances the ability to tune the fluid properties could be very beneficial. Larger bearing clearances and lower preloads produced lower peak displacements by providing greater levels of effective damping. Load-between-pad configurations also produced more ideal rotor behavior than the load-on-pad configurations. Pad configuration and bearing clearance generally had the greatest impact on peak rotor displacement, though preload and lubricant properties produced significant differences under some conditions as well. Abundant nonlinear behavior was found in the cases that experienced a severe rub impact, with chaotic rotor motion and increased vibration amplitudes further describing this behavior. In the cases where the rub impact was removed at low to moderate speeds, synchronous motion was restored to the system. Overall, it was found that slight modifications to bearing design and lubrication could 
provide a simple and cost-effective solution to improving or removing rub impacts in high-speed rotating machinery. 


\section{Chapter 5}

\section{Design of a Gas-Expanded Lubricants}

\section{Bearing Test Rig}

As previous chapters have discussed the performance implications of GELs, there are a number of design considerations that must also be considered when exploring the notion of a new bearing lubrication technology. These design considerations include that of the bearings, bearing housings, bearing seals, lubrication system, monitoring and control system, as well as considerations related to the design and operation of the machine being considered for the new technology. GELs pose a unique challenge in this regard as fully flooded, pressurized bearings would require fairly substantial changes to many of these designs, depending on the application.

When the study of GELs began, it was known that a number of performance models would be available to explore the potential performance of these fluids in high-speed bearings. Therefore the initial goal of the study was to not only study GEL properties and their potential performance in bearings, but to also experimentally measure that 
performance to validate the modeling efforts performed and provide initial proof-ofconcept of the technology to those in the bearing and rotating machinery industries. This approach could then lead to potential commercialization activities surrounding the technology should it be deemed appealing to these industries.

In accordance with this initial plan, a Gas-Expanded Lubricant Bearing Test Rig was designed while considering many of the above important design considerations. Though this test rig was not constructed for reasons that will be discussed later in this chapter pertaining to some of these design considerations, the knowledge gained from this design work was certainly novel in terms of GEL-system design and is worthy of documentation for future test rig or GEL system prototype development. The following sections will detail the design of this bearing test rig, providing its overall design specifications and discussing the most important design considerations that needed to be studied in order to allow for the successful completion and operation of this high-pressure, high-speed machine. The technical limiting factors will also be discussed before moving on to the discussion of the study of these factors in Chapter 6 with another experimental test rig that is to be completed.

\subsection{Test Rig Overview}

The Gas-Expanded Lubricants Test Rig has been designed to experimentally measure the performance of GELs in tilting pad journal bearings. Because GELs involve the use of a tunable lubricant whose original properties can be restored, this test rig would also allow for direct performance comparisons with standard lubricants used in industry. These results could be compared to performance predictions carried out in Chapter 2 as 
the bearing-rotor system modeled in that chapter is of the same design. Key performance metrics of interest with this test rig include bearing power loss, pad temperatures, fluid pressures, and rotor position.

This section provides an overview of the bearing test rig design. A $3 \mathrm{D}$ model of the test section is shown in Figure 5.1. This test section will rest on a concrete pad with dimensions 68 " long, 40" wide, and 26" tall. To provide vibration damping the concrete pad will then rest on a rubber damper mat. A 2" thick, 66" long, and 38" wide carbon steel skid attached to the concrete pad will provide a machined-flat surface for the test rig to ensure proper alignment and support for the heavy, high-speed structure. The use of a concrete pad will also increase the stiffness of the supporting structure, ensuring that structural vibration modes will be located outside of the operating speed range of the test rig. Otherwise, these modes could cause misalignment of the test section and failure of the machine. The test section itself will then consist of a high-speed motor, a double-flex coupling to connect the motor to the shaft, the rotor, two bearings, two high-pressure bearing housings, four bearing seals, and instrumentation. A custom GEL lubrication system will then supply pure oil and GELs to the test section bearings. 


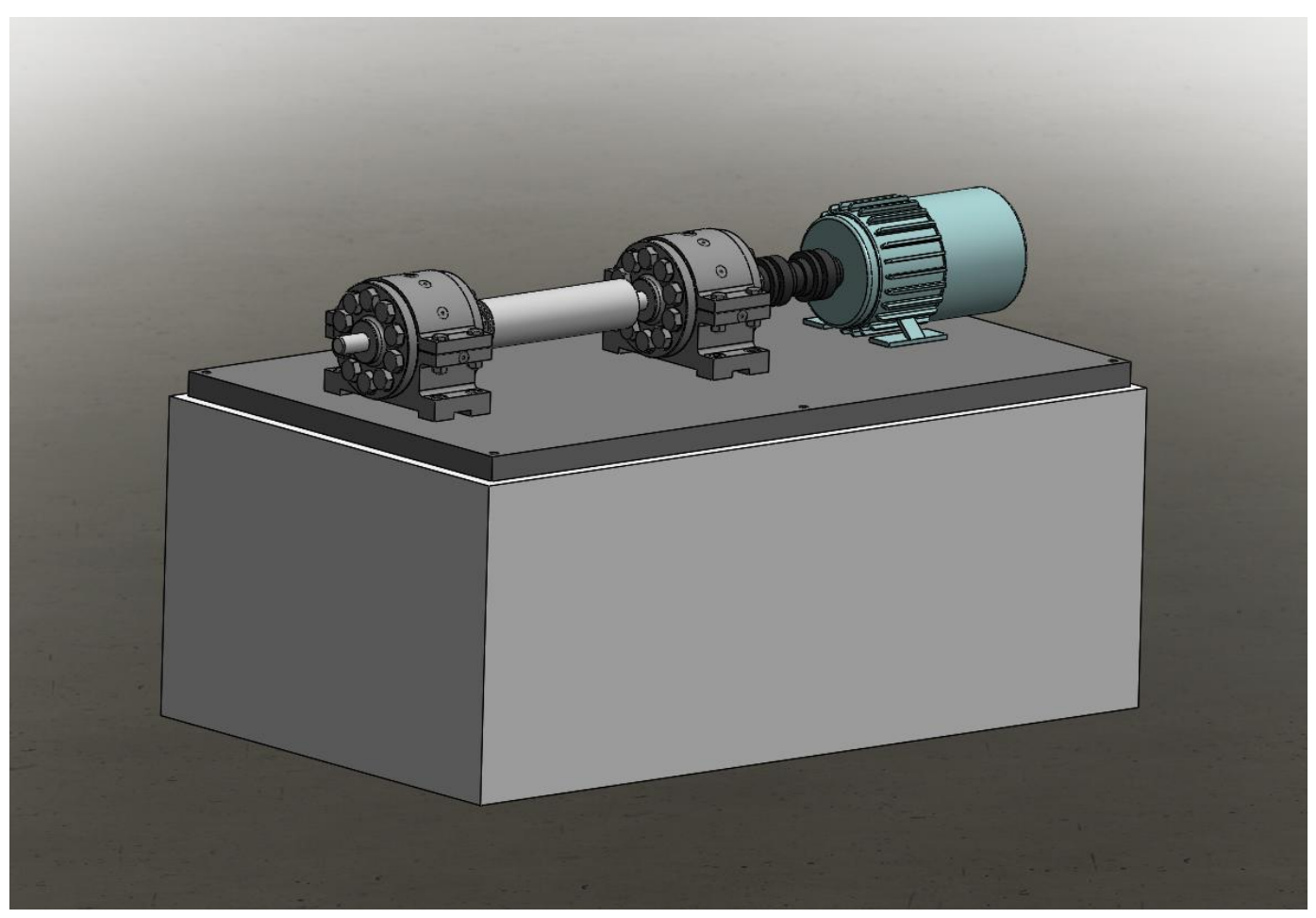

Figure 5.1. A 3D solid model depiction of the GEL Bearing Test Rig test section.

The test rig motor (Figures 5.2 and 5.3) is a Reuland Electric 184T frame motor with a variable speed capability up to $10,000 \mathrm{rpm}$ at $10 \mathrm{hp}$. A number of bearing and seal performance analyses were performed to estimate the power losses of the system due to fluid shear and churning, ensuring that the motor selected would be adequately sized to overcome these losses. This motor is then coupled to the test rig rotor using a Rexnord Thomas Series 71-150 double-flexible disk pack coupling (Figure 5.4). The use of a double-flex coupling allows for up to 2 planes of slight misalignment should it be encountered during assembly or as a result of vibration or other structural shifts. 


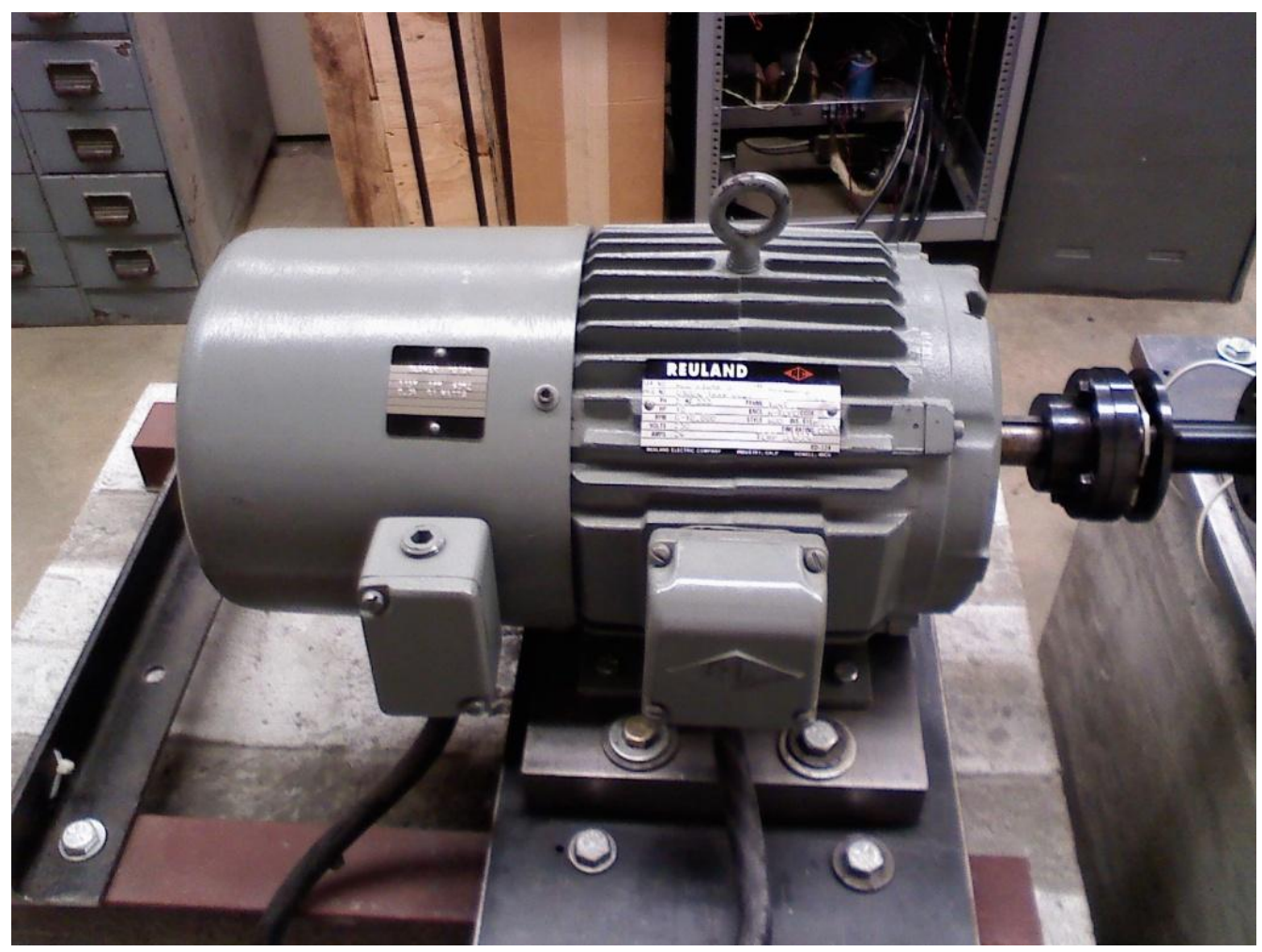

Figure 5.2. Reuland Electric motor to be used with the GEL bearing testbed.

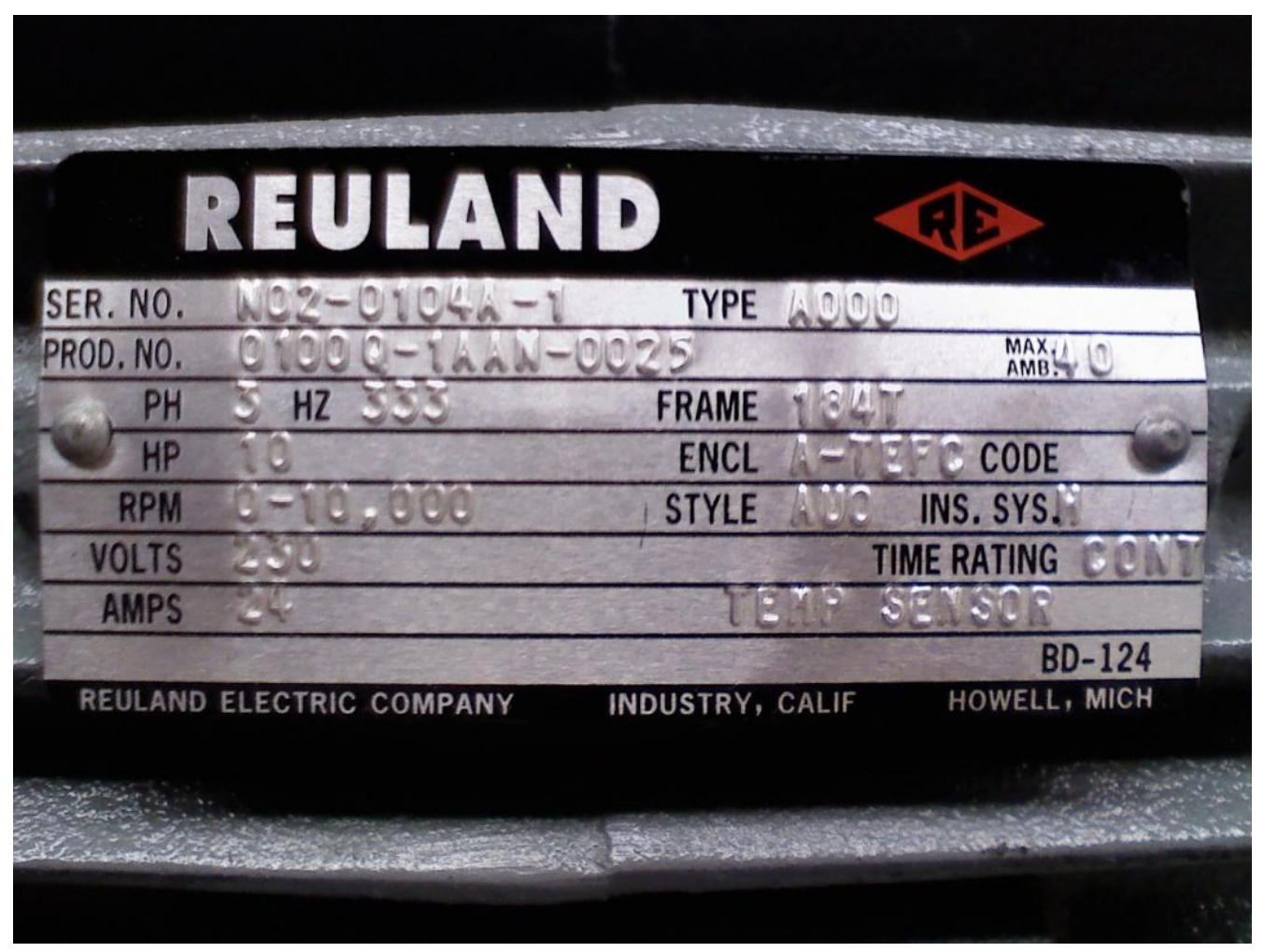

Figure 5.3. Reuland Electric motor specifications. 


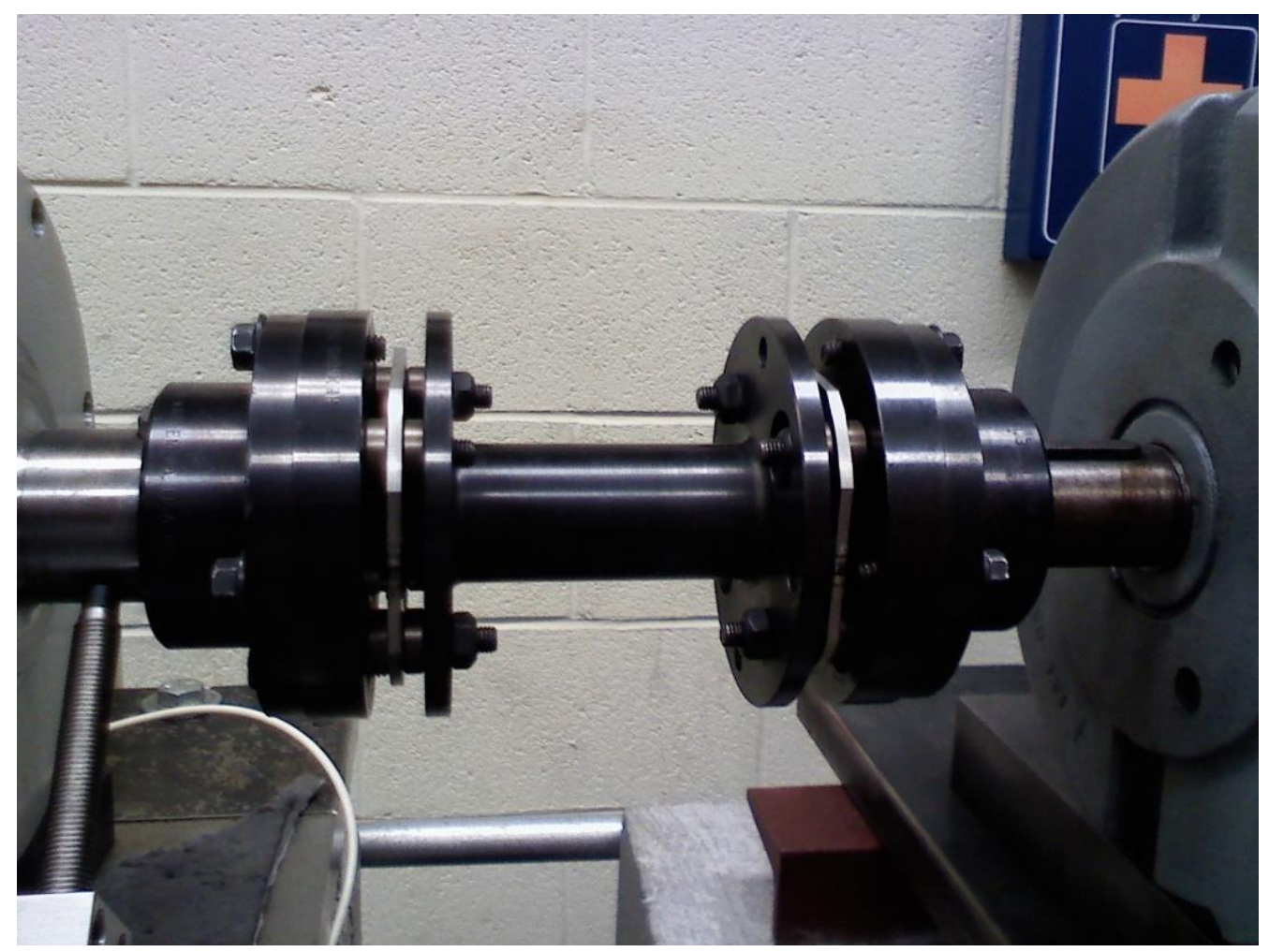

Figure 5.4. Rexnord Thomas 150 SR71 double-flex coupling.

The test rig rotor (Figure 5.5) is designed to have a 44" length, with 14.5" long sections on each end of the shaft having a diameter of 1.5 " and the central 15 " long section having a diameter of 4". The weight of the rotor is approximately $66 \mathrm{lbs}$, or a 43 psi load for single bearing pad with $1 " \mathrm{x} 0.76$ " dimensions. 0.125 " chamfers at each end of the rotor allow for easy coupling attachment and 0.25 " holes drilled into each axial end of the central section allow for the addition of unbalance weights during experiments. The rotor is designed for maximum stiffness to raise the location of the first critical speed of the machine. The large diameter of the central section of the shaft increases the load applied to the test bearings and allows for the addition of an adjustable rolling element bearing at the center of the shaft for further increasing the bearing load during 
experiments. Additional details of rotordynamic analyses performed for the test rig shaft are discussed in section 5.3.

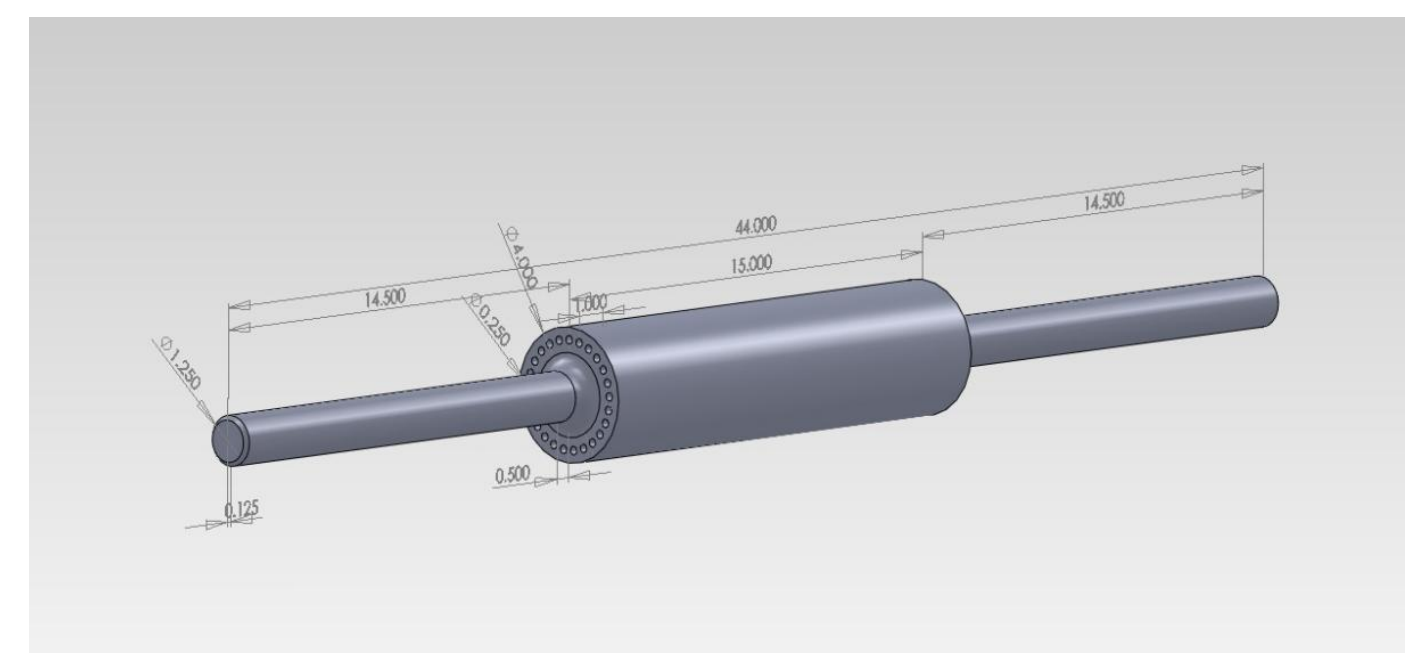

Figure 5.5. GEL bearing test rig rotor.

The bearing housings (Figure 5.6) present one of the most unique aspects of the overall test rig design. As commonly used bearings in the turbomachinery industry are typically operated at pressures up to $40 \mathrm{psi}$ [7], the high pressures required for GELs create an interesting design challenge for the bearing housings and seals. The housings are designed for a maximum pressure of 2,465 psi. This design pressure will allow for a large range of dissolved $\mathrm{CO}_{2}$ mass fractions in the GEL that could be studied at a wide range of operating temperatures. The split-housing design will also allow for easy assembly and disassembly of the test sections. Details on the design of the bearing housings for these elevated pressures are discussed in section 5.2. These housings are equipped with ten high-pressure ports for routing thermocouples to each of the five bearing pads for measuring pad temperatures in both load-on-pad and load-between-pad bearing configurations. Figure 5.7 provides a cross-sectional view of the inside of the 
bearing housing. Details on the design of the bearings used for this test rig are found in Table 2.3.

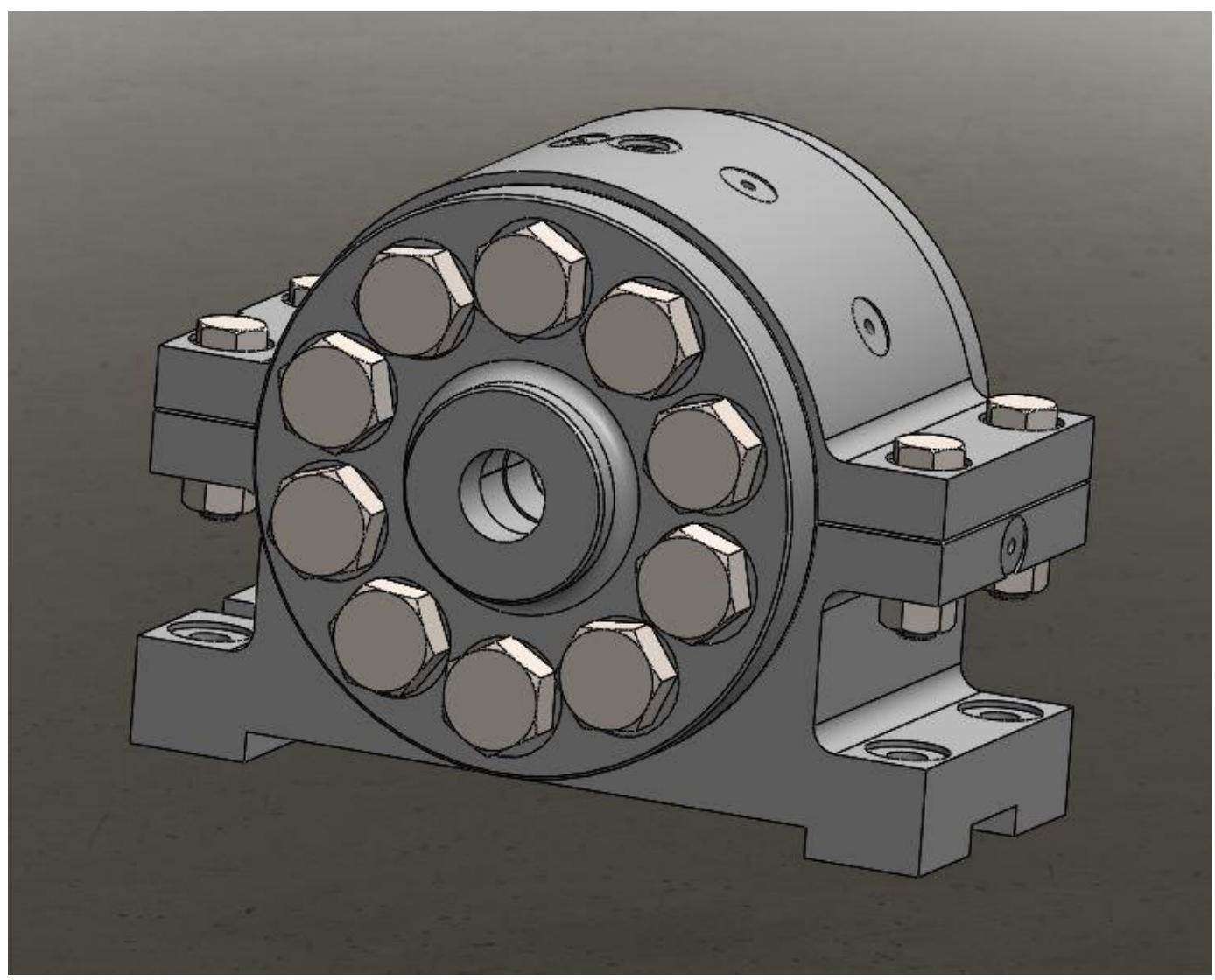

Figure 5.6. High-pressure bearing housing for the GEL bearing test rig. 


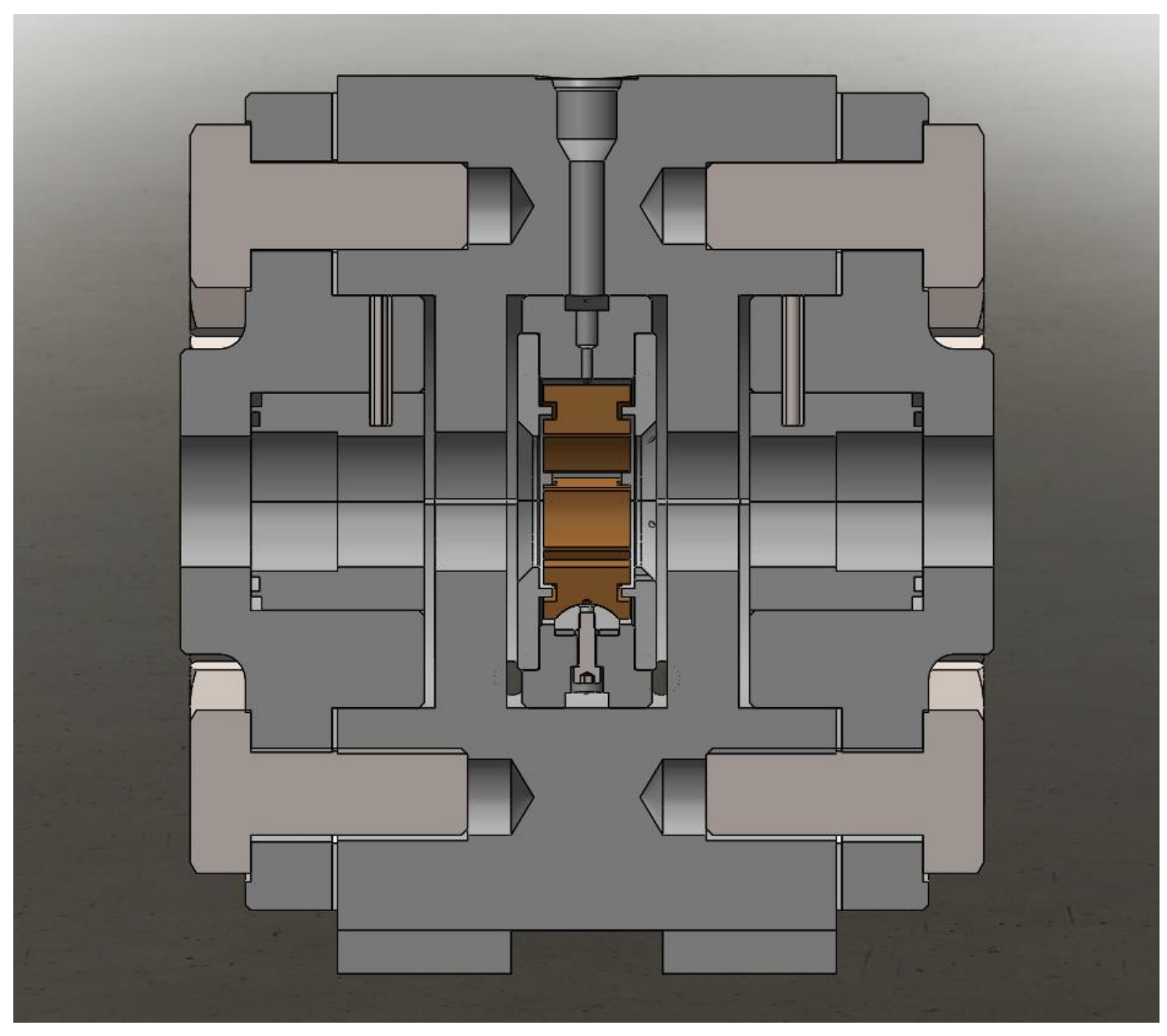

Figure 5.7. High-pressure bearing housing cross-section.

\subsection{Bearing Housing Finite Element Analysis}

As the bearing housings were designed for pressures up to 2,465 psi, it was necessary to perform a finite element analysis of the bearing housing to determine a number of important housing design parameters including the locations of peak stresses, the housing material that would be necessary to facilitate such a design, and the size and number of bolts required to securely hold the various sections of the bearing housing in place without significant deformation taking place. 
Figure 5.8 shows the finite element model constructed for the bearing housing using the mechanical modeler in ANSYS Workbench. This model utilized a fine mesh and a symmetrical boundary condition in the axial direction to reduce both the total number of nodes and the convergence time. Forces applied to the housing include the internal pressure of 2,465 psi as well as bolt preloads calculated using [117]. Other boundary conditions utilized include fixed displacements along the symmetry plane, on the bottom of the housing, and on the floor bolt threads, bonded contact surfaces between the bolt heads/threads and contacting surfaces, and sliding friction contact surfaces between the different housing sections.

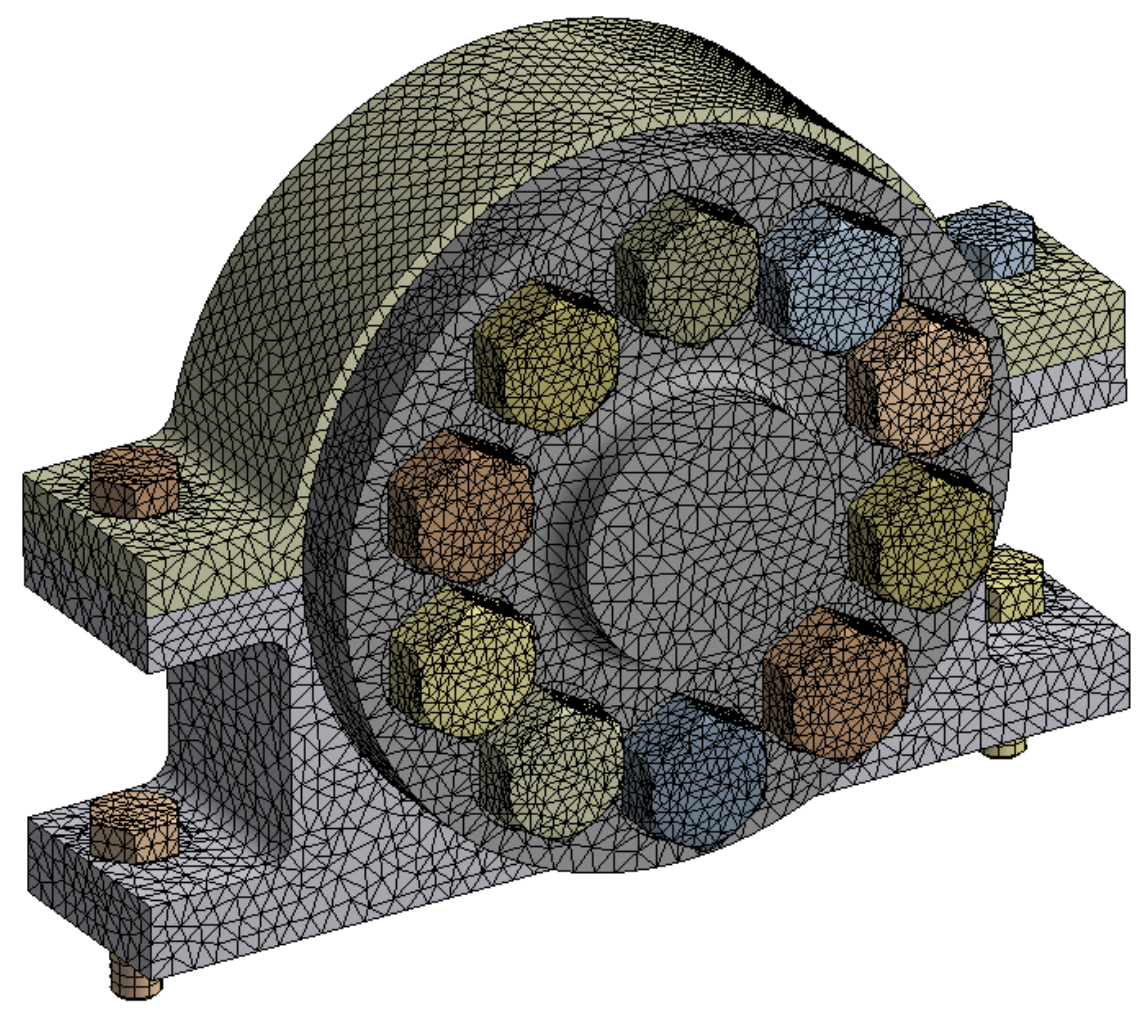

Figure 5.8. Finite element model of the bearing housing. 
The peak stresses of the bearing housing are shown in Figure 5.9 to be approximately 70,000 psi. Using a proper factor of safety, it was found that when using a higher strength steel such as a 15-5 precipitation-hardened stainless steel that the peak stresses would equal less than $58 \%$ of the yield strength of the material, essentially providing infinite fatigue life at the design pressure chosen. Considering both these strength requirements as well as the corrosion resistance that would be required for $\mathrm{CO}_{2}$ compatibility, the 15-5 precipitation-hardened stainless steel was chosen as the material for the bearing housings.

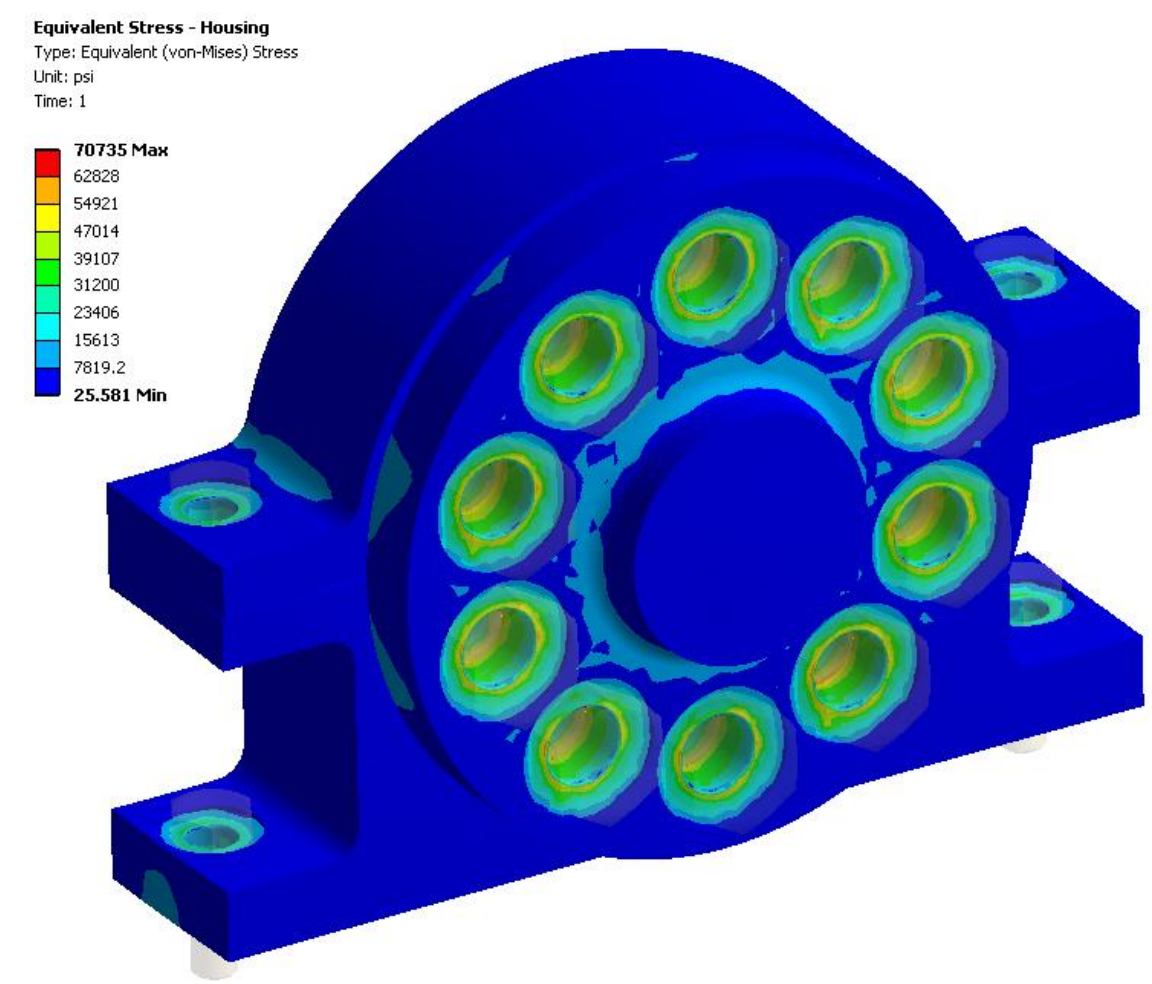

Figure 5.9. Peak stresses in the high-pressure bearing housing.

Peak stresses in the bolts were also evaluated to determine the proper size, number, and type of bolts that needed to be used (Figure 5.10). It was found that the peak bolt cross-sectional stresses remained below 55,000 psi at design pressure. This led to the 
conclusion that the Grade 8 bolts selected for the bearing housing would be adequate for these stresses due to their large yield strength of 130,000 psi. Housing separation (Figure 5.11) and deformation (Figure 5.12) were also evaluated, finding that peak separations and deformations were 0.00075 " and 0.0015 ", respectively. These results led to the conclusion that while adequate contact and minimum deformation could be maintained with this design, the use of static seals at the bolted joints would be necessary to prevent leakage from these joints. PTFE gaskets have been chosen to provide this sealing action.

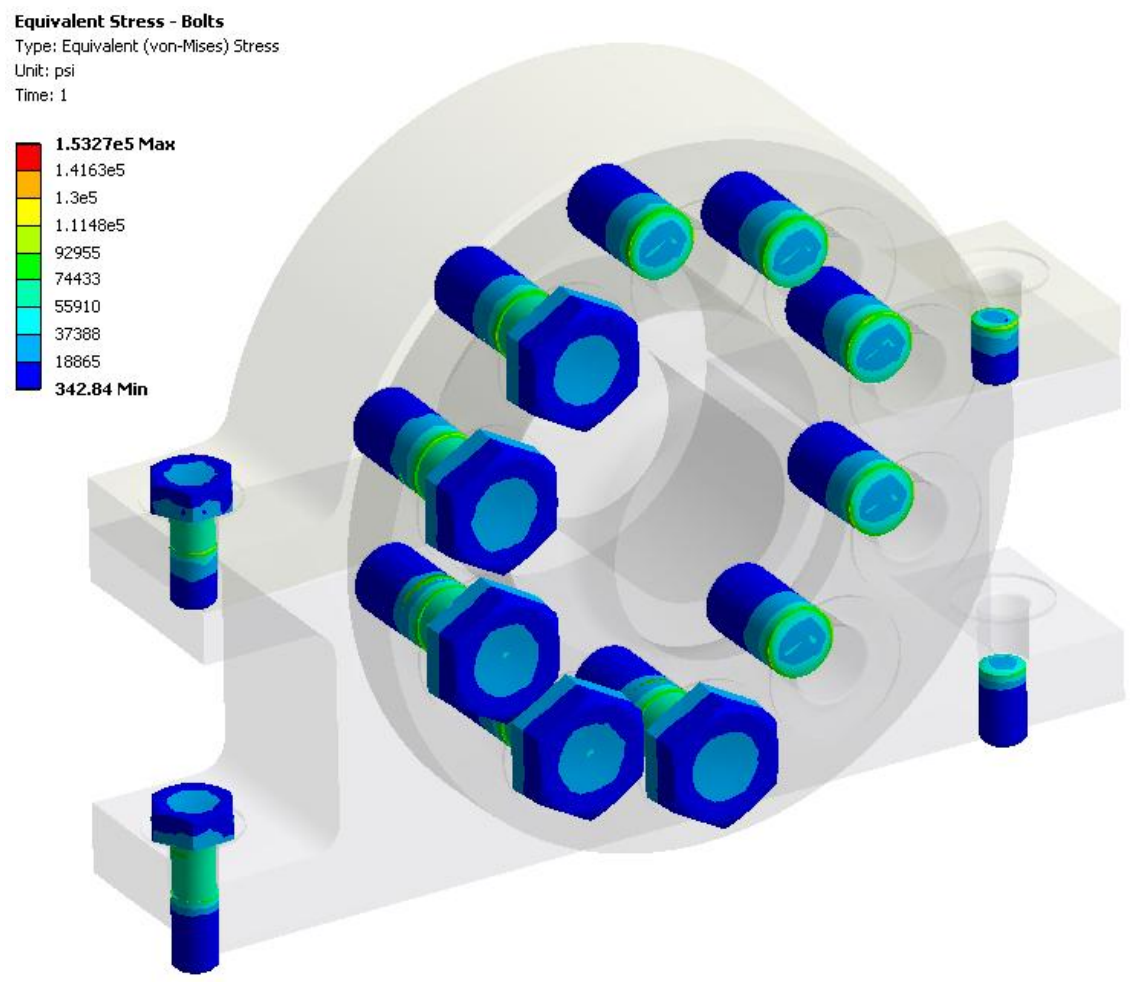

Figure 5.10. Peak stresses in the housing bolts. 


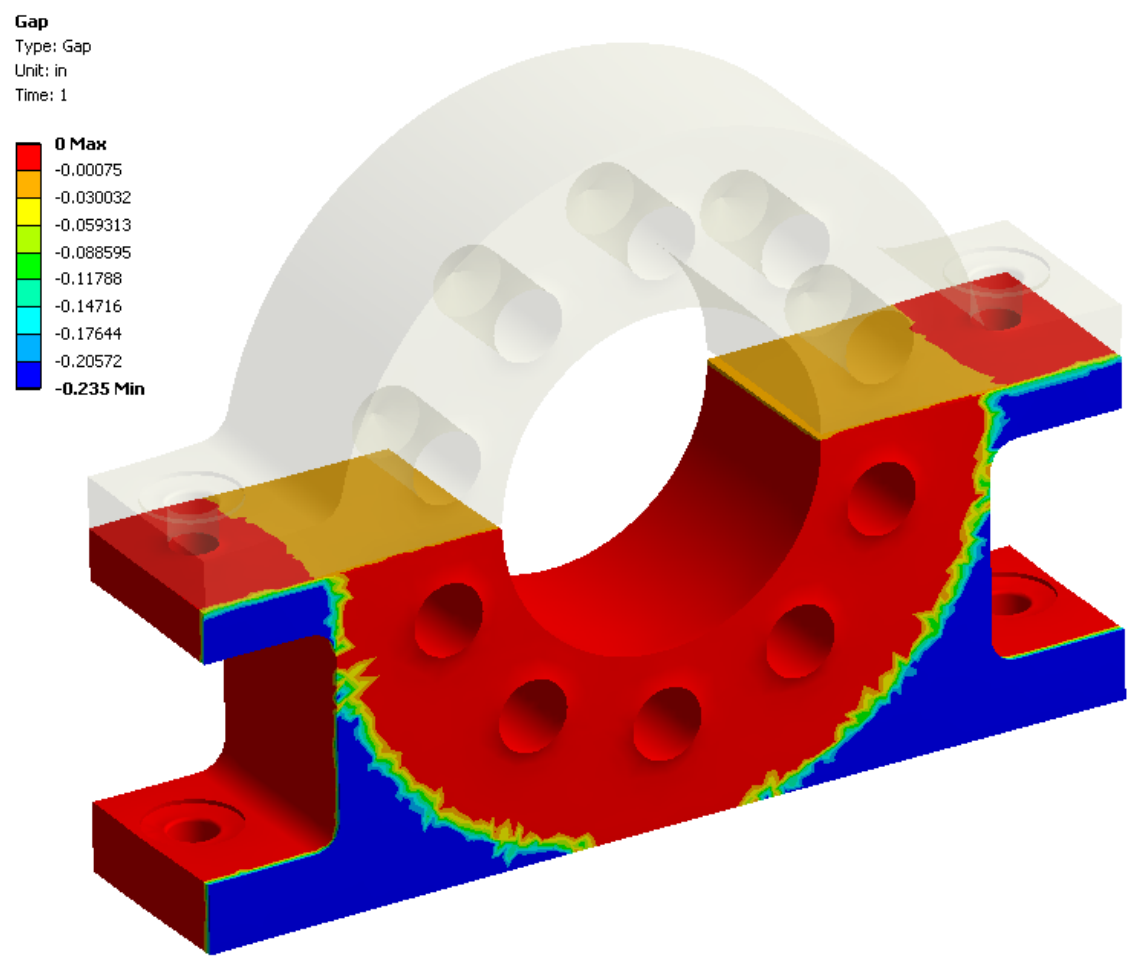

Figure 5.11. Separation between the housing surfaces at design pressure.

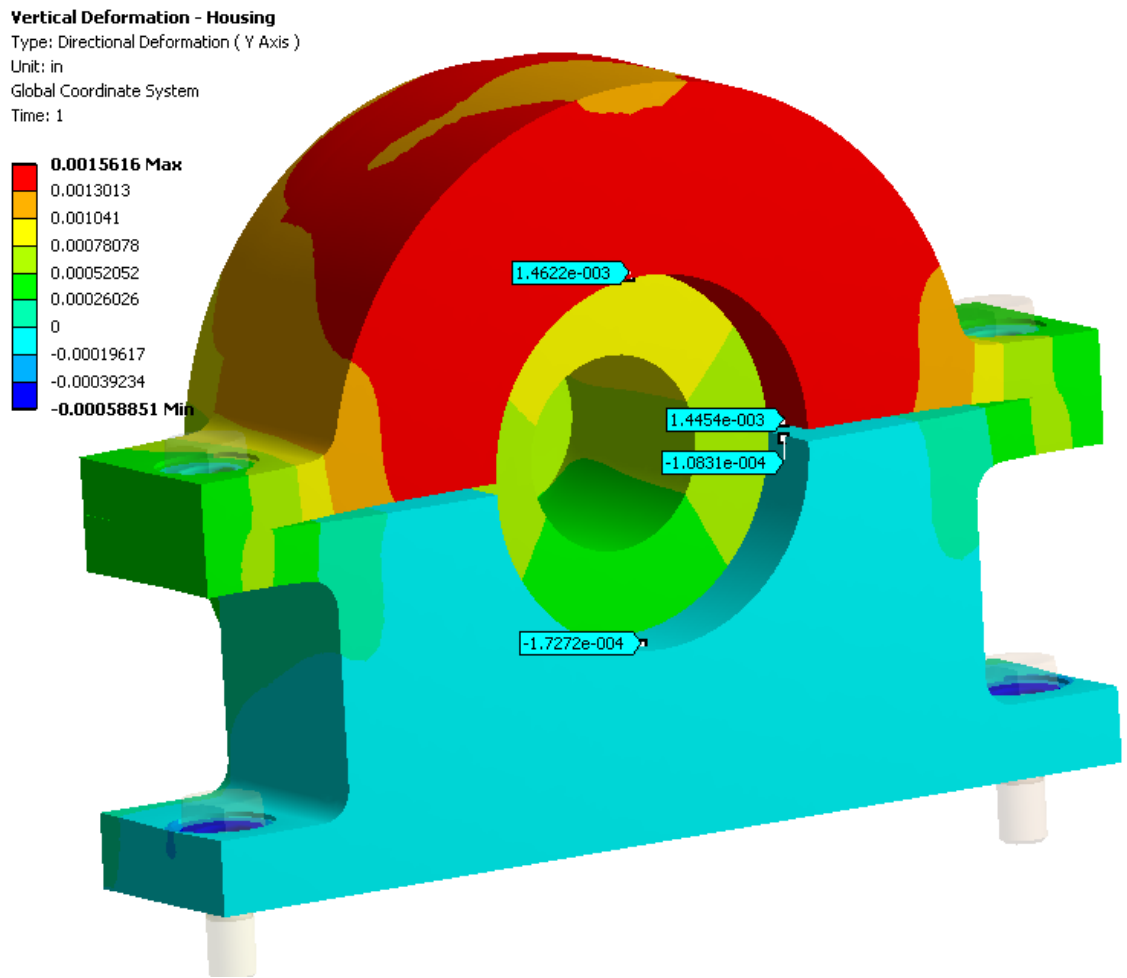

Figure 5.12. Deformation of the bearing housing at design pressure. 


\subsection{Rotordynamic Analysis}

To ensure adequate rotordynamic performance of the test rig for the speed range desired, it was necessary to perform a standard rotordynamic analysis of the machine. This analysis took into account both the performance of the machine bearings as well as the high-pressure floating bushing seals described in section 5.6 and was performed using methods described in section 3.2.3.

The first step of the analysis was to create a critical speed map of the system (Figure 5.13). This analysis revealed that there existed two critical speeds in the $0-10,000 \mathrm{rpm}$ operating range selected for the test rig, shown in the Figure by the intersection of the $\mathrm{K}_{\mathrm{xx}}$ and $\mathrm{K}_{\mathrm{yy}}$ bearing stiffness coefficients with the two lines denoting the critical speeds in the operating range. The estimated critical speeds were located at approximately 5,000 and $8,000 \mathrm{rpm}$.

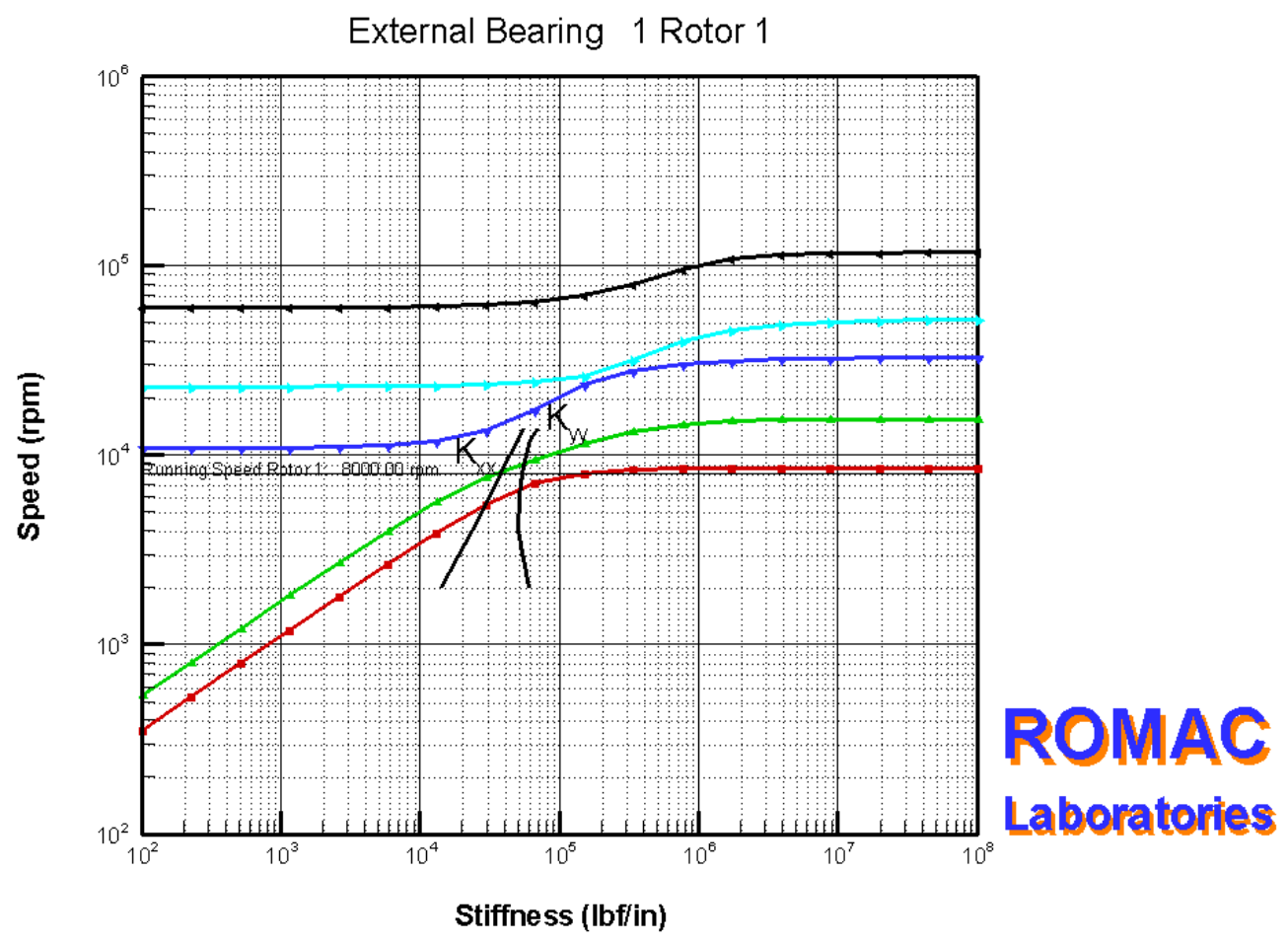

Figure 5.13. Critical speed map of the GEL bearing test rig. 
An undamped mode shape analysis (Figure 5.14) predicted the shapes of the two critical speeds to be a $1^{\text {st }}$ bending mode (shown in red) and a gyroscopic mode (shown in green). This then led to the portion of the analysis that used the bearing and seal coefficients as inputs to the model for assessing mode stability, damped mode shapes, and unbalance response. Two scenarios were considered for this portion of the analysis: one scenario where the floating bushing seals were assumed to be floating with the eccentric motion of the shaft, and a second scenario where the seals were assumed to be locked into position which would further enhance their effects on the rotordynamic performance of the machine.

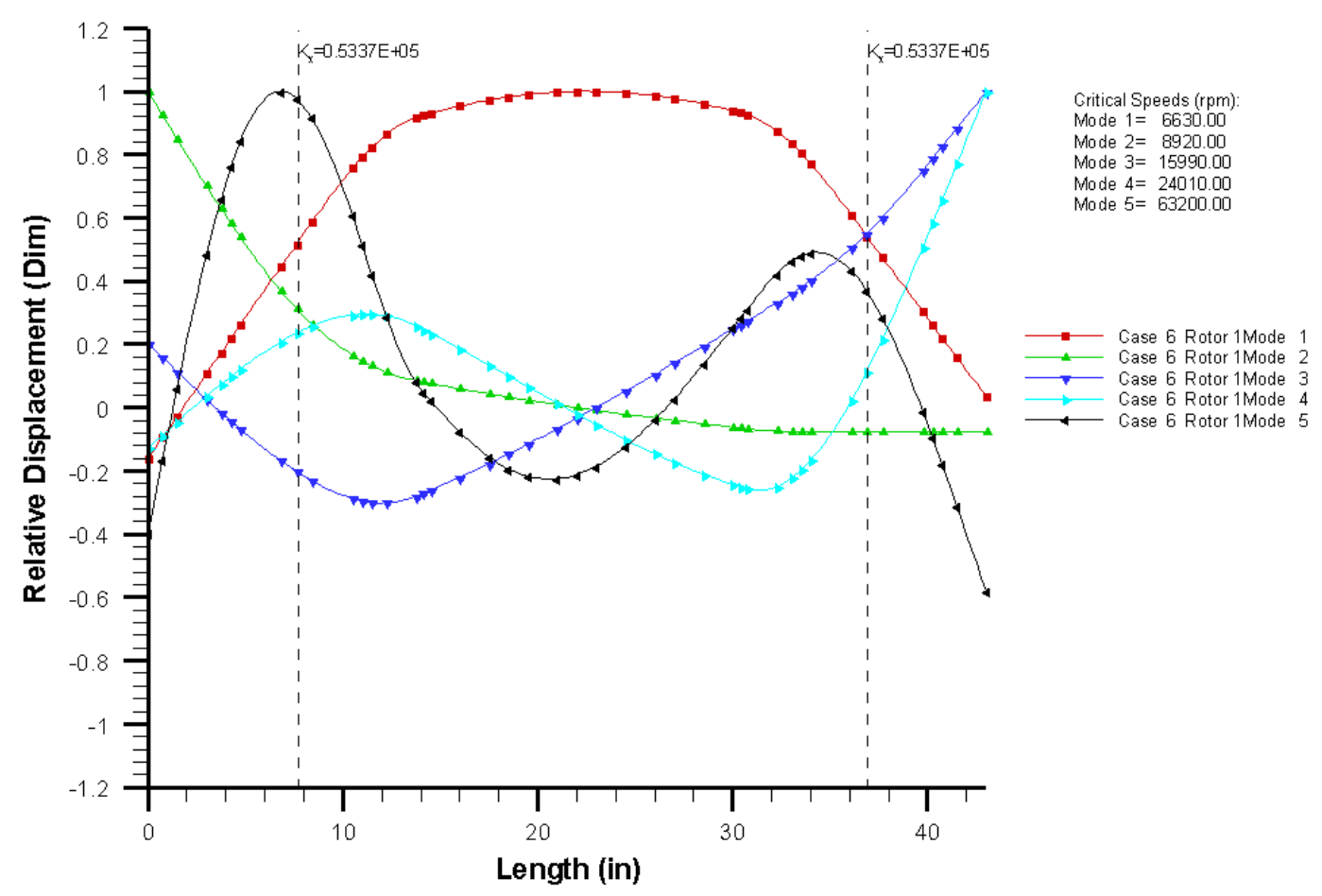

Figure 5.14. Undamped mode shapes of the bearing test rig rotor.

The stability analysis revealed some interesting results when comparing the two cases considered. For the scenario in which floating seals were assumed, the two modes in the 
operating range were found to be well damped with the prediction of large log decrement values for these modes, indicating a stable operation of the machine while passing through these modes. The second scenario, in which the seals were assumed to lock into position, predicted much lower values of log decrement. As discussed in Chapter 3, values above zero are indicative of a stable system, though commonly used industry standards such as API 617 [99] require a value above 0.1 to include a factor of safety. For the modes predicted by this analysis log decrement values were calculated as low as 0.02 , leading to the prediction of a stable system but one that must be carefully monitored when brought up to higher speeds. Seal lockup speed calculations were also performed in accordance with Allaire and Kocur [118], indicating that the seals were likely to lock up between speeds of 500 and 1,000 rpm at typical operating pressures. This further emphasized the care that needed to be taken when starting up the machine and pressurizing the bearings.

Damped mode shapes and unbalance response were the final rotordynamic characteristics assessed for the test rig design. Taking into account the effects of the bearing and seal stiffness and damping coefficients, the mode shape of the first critical speed was found to change from a $1^{\text {st }}$ bending mode to a conical mode shape (Figure $5.15)$. 


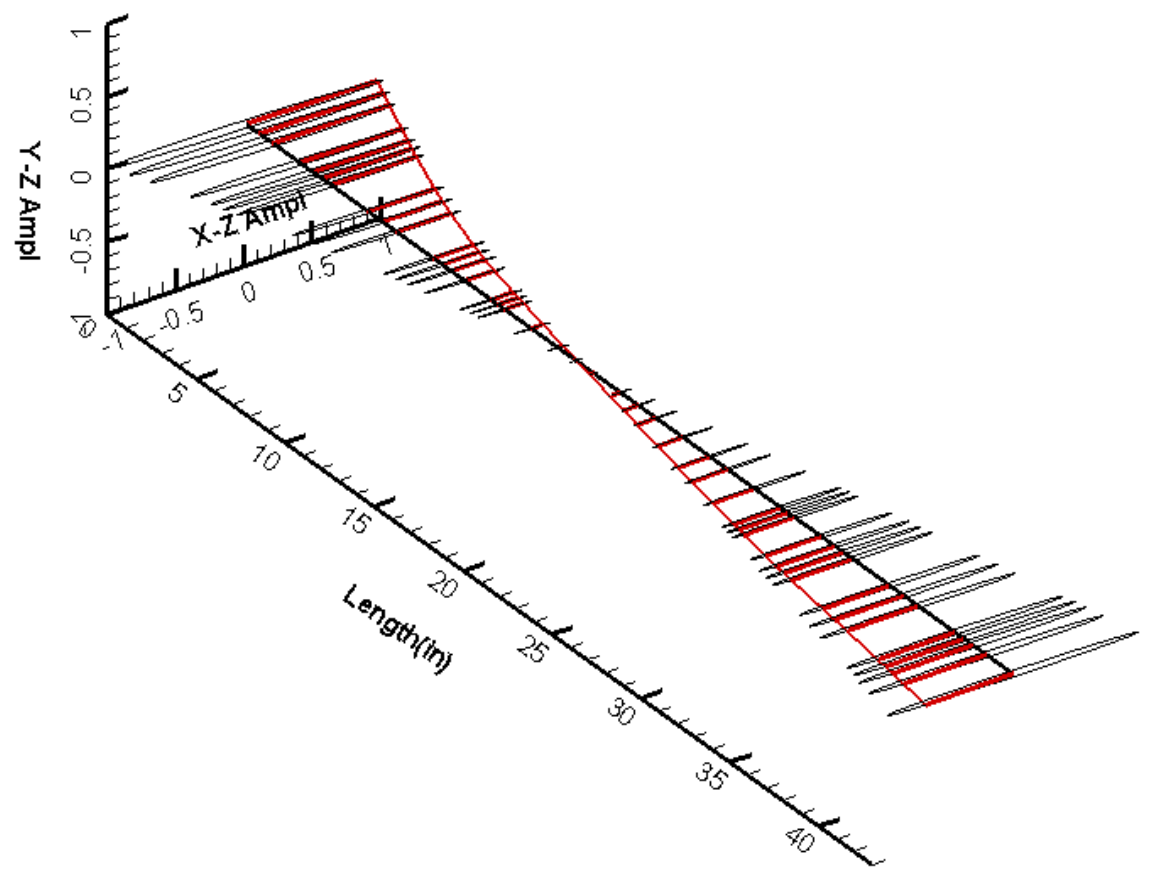

Figure 5.15. Damped mode shape of the first critical speed.

The unbalance response of both modes in the operating range was analyzed for both scenarios considered in the stability analysis. The second mode of the first scenario produced the largest peak displacements of the analyses performed (Figure 5.16), indicating that peak displacements would remain below the bearing and seal clearances chosen for the test rig design. 


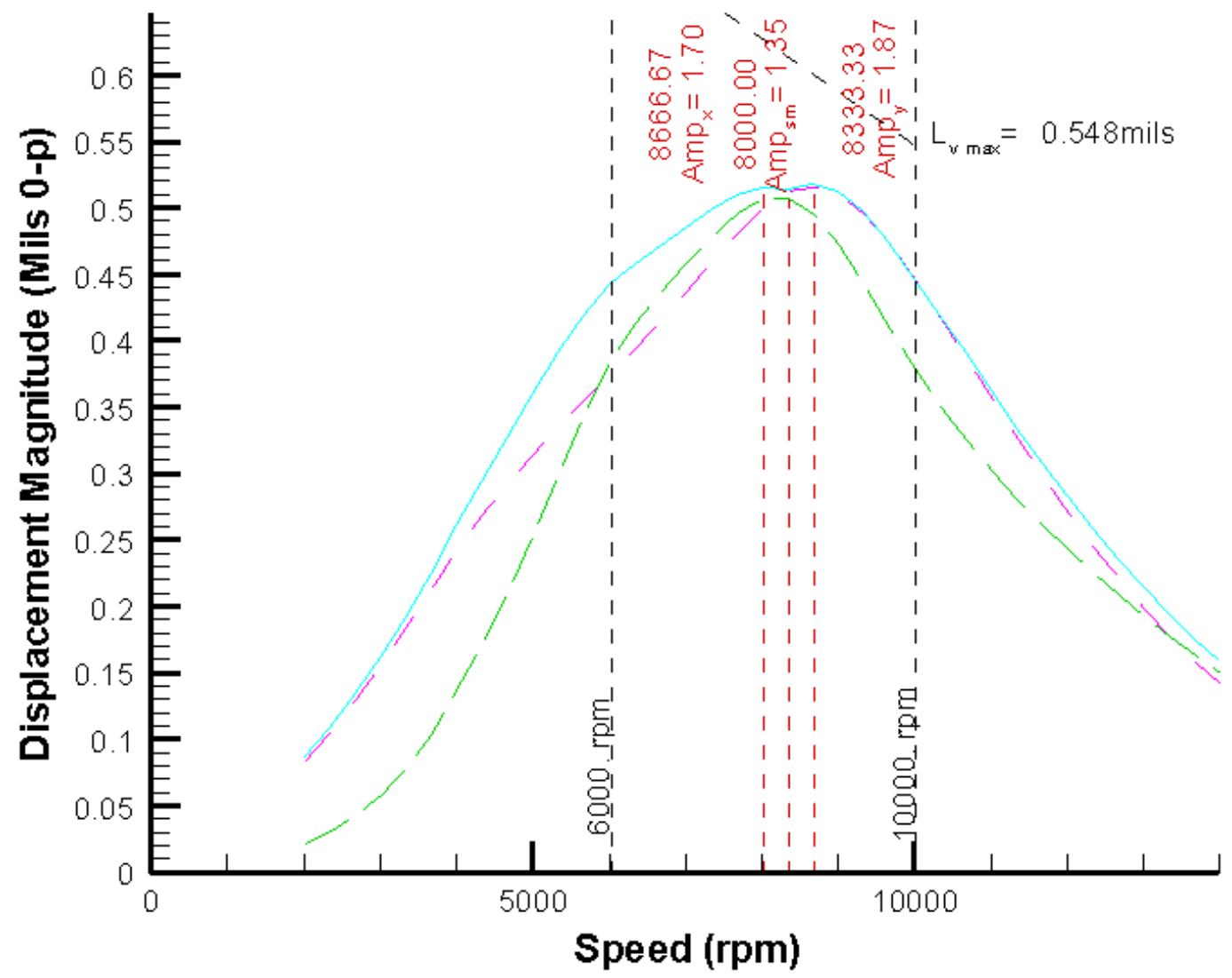

Figure 5.16. Unbalance response of the second mode in one of the test rig bearings.

Overall, this analysis concluded that while the stability and unbalance response of the machine would remain at levels considered safe for operation for a majority of the operating conditions considered, care must be taken when operating the test rig at the highest speeds and pressures considered for experiments to ensure that no damage would come to the machine.

\subsection{Lubrication System}

The GEL lubrication system design for the bearing test rig also presented a unique design challenge. This system required accurate control of the GEL composition, thus requiring excellent control over flow rates, pressures, and temperatures in the fluid to 
ensure a single-phase, homogenous mixture to the bearing test section. These factors, along with system design pressure and flow rate were considered as the lubrication system was designed and assembled in partnership with the manufacturer American Design \& Manufacturing. The delivery system has since been received and is shown in Figure 5.17.

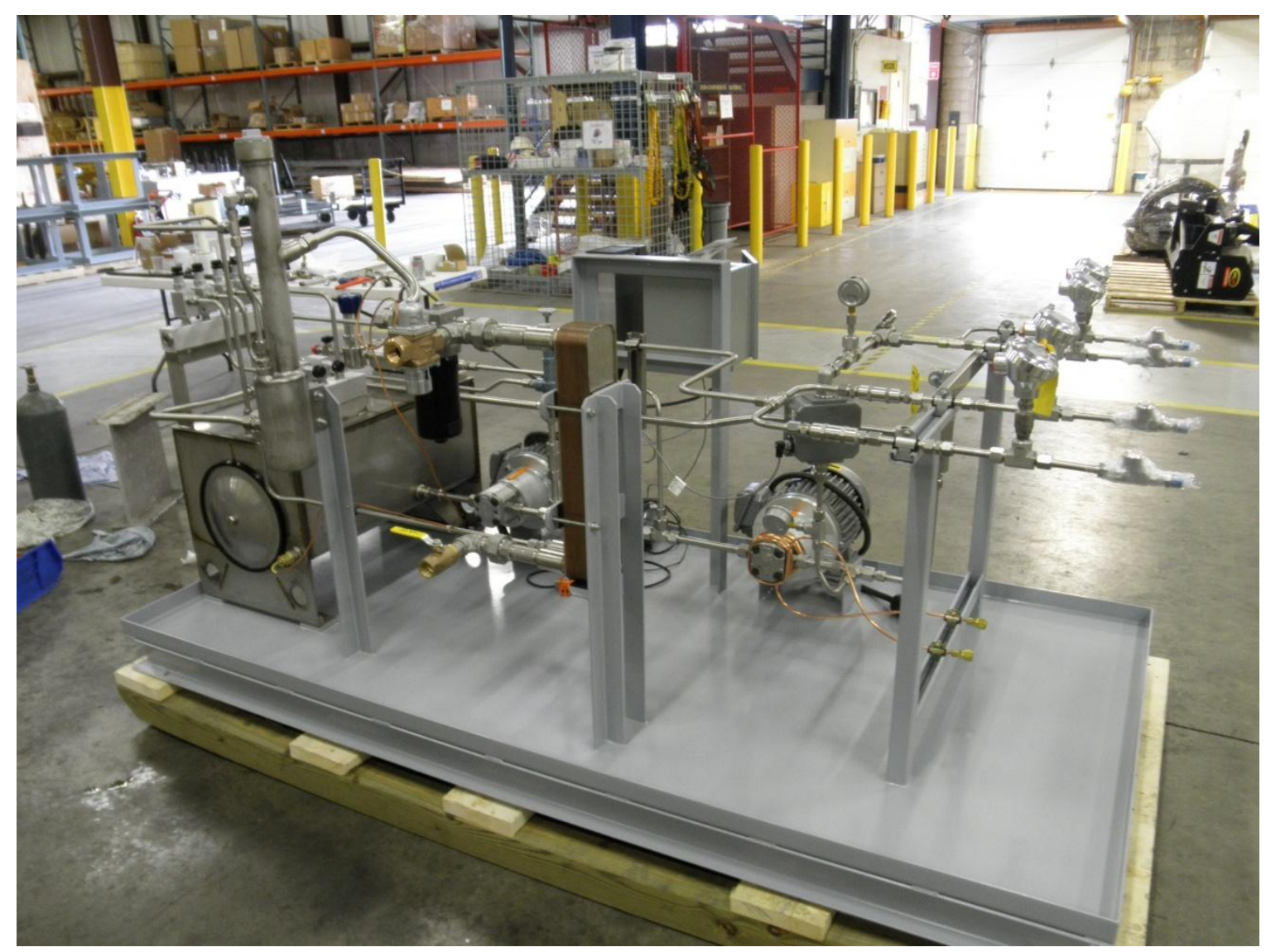

Figure 5.17. The GEL delivery system for the bearing test rig.

This system consists of a two-stage gear pump design (Sauer aluminum gear pumps), allowing for both single and multi-phase lubricants including GELs to be delivered to the test section at pressures up to $2,500 \mathrm{psi}$ and flow rates of $2 \mathrm{gpm}$. The first stage pump flow and pressure are controlled by a variable frequency drive and an adjustable relief 
valve providing return flow to the lubricant reservoir. The reservoir holds 20 gallons of polyol ester lubricant with an initial viscosity of $53 \mathrm{mPa} \cdot \mathrm{s}$ at $40^{\circ} \mathrm{C}$. This lubricant was chosen is it was found to form miscible, single-phase mixtures when combined with carbon dioxide, whereas other synthetics may exhibit miscibility limits under many operating conditions [6]. The larger initial viscosity also allows for a wider range of GEL viscosities to be studied. Carbon dioxide is fed into the lubricant flow between the two gear pumps after passing through a pressure regulator and a mass flow controller (Brooks SLA Series). The second stage gear pump then provides pumping and mixing of the GEL mixture as it is delivered to the test section. Test section inlet and outlet pressure and temperatures are measured along with total flow rate using pressure transducers (Omega Engineering), thermocouples (TTEC 8006 Series RTD), pressure/temperature gauges, and a flow meter (Hedland H600 Series).

After exiting the test section, the fluid passes through a series of adjustable cartridge relief valves (Sun Hydraulics) to control the back pressure of the fluid in the test section. These will allow for bearing pressures ranging from 50 psi to the design pressure of 2,465 psi. The flow then passes through a 30,000 BTU brazed plate heat exchanger (ITT) followed by a custom degasser for removing any remaining carbon dioxide in the fluid following depressurization as well as a 10 micron oil filter (Schroeder). The fluid then returns to the lubricant reservoir and carbon dioxide emissions are routed outdoors. Though this system requires the use of a fresh carbon dioxide stream that is then lost to the atmosphere, it is anticipated that other designs could be utilized involving highpressure lubricant reservoirs or separate carbon dioxide capture and recompression 
equipment to allow for reuse of the carbon dioxide in the system. An open-loop design was chosen for this experimental system to minimize cost.

\subsection{Data Acquisition and Instrumentation}

A variety of performance data can be collected from this bearing test rig. The overall data acquisition system, provided by National Instruments, will consist of a multi-slot chassis, a series of input and output modules for sending and receiving voltage and amperage signals to and from instrumentation and control units, a computer equipped with LabView software for controlling the system and logging data, and the data collection instrumentation. Ten thermocouples (Omega Engineering) will provide pad temperature data for each of the five pads in the two test bearings at multiple bearing configurations. These instruments will be routed into the bearing housing and securely sealed using high-pressure fittings from High Pressure Equipment Company. Pressure transducers (Omega Engineering) at the fluid inlets and outlets to the test bearings will monitor and allow for the control of fluid pressures in the bearings.

Power loss in the system due to fluid shear will be measured by multiplying the operating speed by the measured torque of the system. Torque will be measured using a torque transducer with a $2 \mathrm{~N} \cdot \mathrm{m}$ range (Honeywell 1701 Model). This range was determined via the previously mentioned bearing and seal performance predictions that were performed for the test rig. The motion of the rotor will also be measured using a series of proximity probes (Micro Epsilon). The LabView-equipped computer will then collect and store all of this information while providing output control signals to the 
carbon dioxide mass flow controller, the first-stage gear pump variable frequency drive, and the variable frequency drive of the test rig motor.

\subsection{Bearing Seals}

The rotary seals for the pressurized bearing housings produced the most challenging aspect of the GEL bearing test rig design. Based on the judgment of the mechanical engineers collaborating on this project, a non-contacting, low-clearance, floating bushing seal was chosen as the initial design for the test rig seals (Figure 5.18). These seals are commonly used in the turbomachinery industry and come with the benefits of low leakage, minimal power losses due to the lack of contacting surfaces, and enhanced rotordynamic performance due to the floating nature of the seal $[118,119]$. Circular or rectangular circumferential grooves can also be added to the seal face to reduce leakage and enhance rotordynamic performance, though depending on the design and application these benefits can also come in the form of tradeoffs [118].

The design of this seal includes a 2" total axial length, with the length broken down into two 1 " sections. The section closest to the bearing has an extremely tight 0.001 " radial clearance to help minimize leakage. The outer 1" length of the seal has an increased clearance to then reduce the amount of spraying of any leakage that should occur. The front of the seal contains a groove for the placement of a PTFE o-ring that will serve as the static seal between the axial face of the bushing seal and the inner wall of the bearing housing. PTFE was chosen for the o-ring material as it has a very low coefficient of friction, further allowing the seal to float radially, as well as superior sealing and material compatibility in carbon dioxide applications. For ease of installation, the seal is 
split vertically and held intact with four socket head cap screws. An anti-rotation pin on the top of seal will prevent the seal from rotating inside the bearing housing.

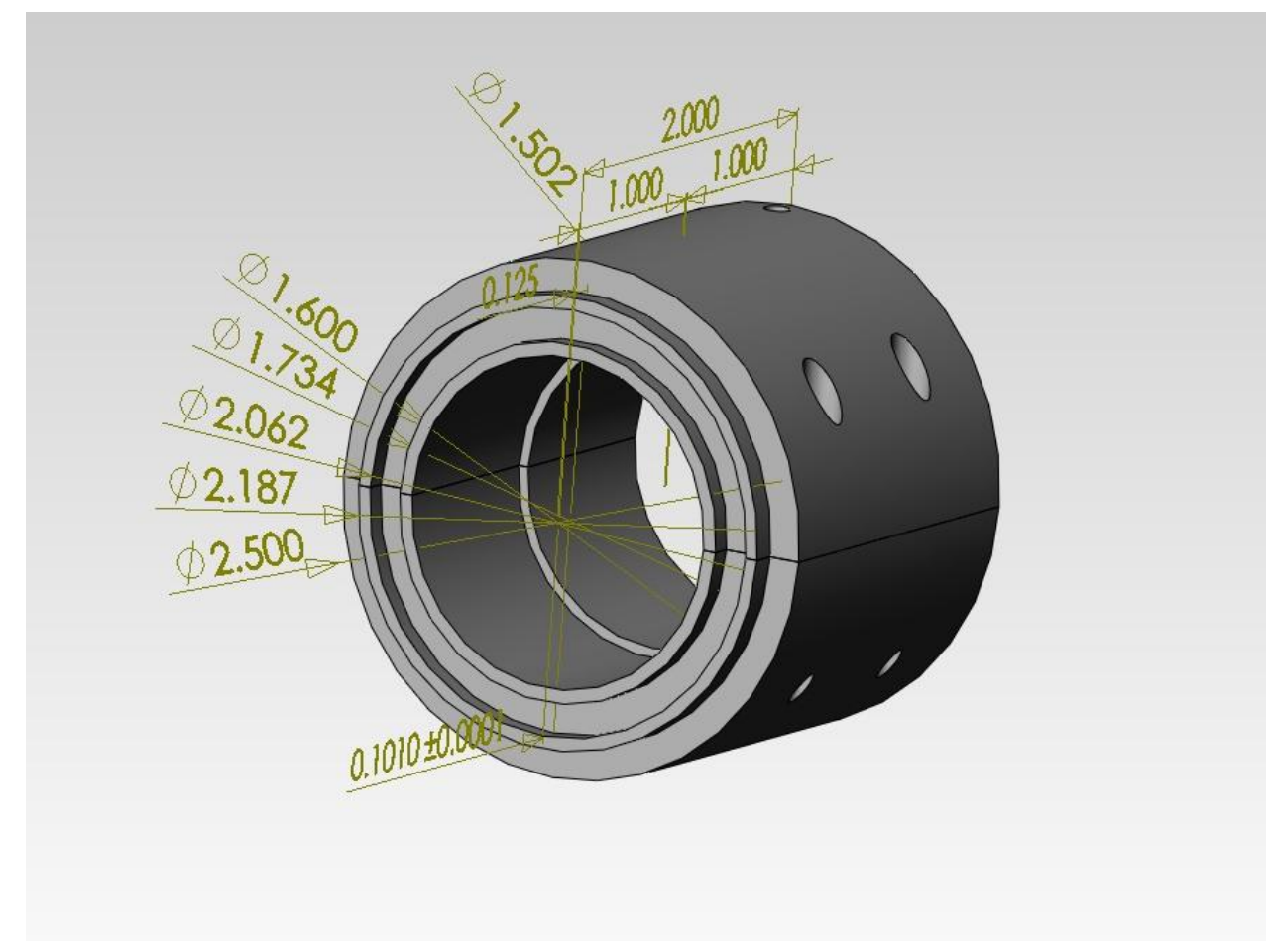

Figure 5.18. Floating bushing seal for the high-pressure bearing housings.

The leakage performance of the seal was predicted using a variety of models available in the literature and from collaborators. These models employed multiple techniques for calculating seal leakage including bulk flow theory, Reynold's Equationbased finite element analysis, and theory of flow through concentric orifices [82, 119121]. However, due to the variety of techniques used in these various seal modeling approaches, estimates for expected leakage were quite varied as well, with predicted leakage rates ranging from $1 \mathrm{cc} / \mathrm{s}$ to $17 \mathrm{cc} / \mathrm{s}$ to $23 \mathrm{cc} / \mathrm{s}$, depending on the method. If 23 $\mathrm{cc} / \mathrm{s}$ of leakage were in fact encountered with the test rig, this would be disastrous for the experiments planned. 
Given the uncertainty surrounding both the expected leakage and rotordynamic performance of the floating bushing seals, a number of other seal technologies were also explored for their viable use with the GEL bearing test rig. These seals included lip seals [122, 123], labyrinth seals [124-134], a variety of annular seals including bushing, ring, honeycomb, and hole pattern seals [135-146], centrifugal motion seals [47, 147, 148], and mechanical seals [149-163]. However, these sealing technologies also faced a number of technical hurdles to being implemented in GEL environments including limited pressure and/or speed ratings (lip seals, centrifugal motion seals), limited to no use or understanding of their performance in high-pressure liquid environments (labyrinth seals, many annular seals, mechanical seals), and excessive technical demands and monetary cost associated with their use (mechanical seals).

Hence, after considering the uncertainty surrounding the current seal design, the technical hurdles faced in utilizing other types of bearing seals, as well as other nontechnical logistical issues, it was decided that instead of building the GEL Bearing Test Rig that a GEL Seal Test Rig would be designed and assembled to begin answering the important questions surrounding the use of various seal types in high-pressure GEL environments. Additional details on the design and assembly of the GEL Seal Test Rig will be discussed in Chapter 6.

\subsection{Summary}

A Gas-Expanded Lubricants Bearing Test Rig has been designed which includes a number of novel designs for GEL-compatible bearing lubrication system components including high-pressure bearing housings for containing these fluids and a fully 
controllable GEL delivery system for controlling the properties of the lubricant in real time. All of the ancillary components for the system have been specified and a number of performance, finite element, and rotordynamic analyses have been performed to ensure its proper design and operation. Due to the uncertainty surrounding the GEL seals for this test rig the assembly has been delayed until important questions regarding GEL seal performance can be answered. However, a great deal of useful information has been obtained as a result of the bearing test rig design process, and it is hoped that the test rig will be assembled in the future to provide important experimental data on the performance of GELs in tilting pad journal bearings. 


\section{Chapter 6}

\section{Design and Assembly of a Gas-Expanded}

\section{Lubricants Seal Test Rig}

As discussed in Chapter 5, the need to better understand seal performance in GELlubricated bearings drove the need to develop a test rig for studying that performance. The knowledge gained from designing the GEL bearing test rig and assessing a wide range of high-pressure seals in the literature aided in this development. This chapter details the design of the Gas-Expanded Lubricants Seal Test Rig that will be used to study a variety of seals used for low-pressure liquids, GELs, multi-phase mixtures, and pure gases. A variety of bulk flow, computational fluid dynamics, and hybrid methods have been developed to model the performance of these seals [164-169]. The GEL Seal Test Rig will also serve to experimentally validate these methods as they are further developed and improved in the future. The knowledge gained from this test rig will then guide future GEL seal and other component designs while providing important experimental data to the academic and industrial communities on high-pressure liquid 
and multi-phase seal performance, information that is far less common than that of gas or low-pressure liquid seals.

\subsection{Test Rig Overview}

This section provides an overview of the design of the Gas-Expanded Lubricants Seal Test Rig. A 3D solid model of the test rig is shown in Figure 6.1. This test rig will rest on the concrete pad discussed in section 5.1. A flat-machined, 1.25" thick carbon steel plate will then support the test section and allow for ease of alignment of the test section components. The test section consists of a spindle motor, a series of single-flex couplings, the rotor, ball bearings with support structures, and a housing assembly with attached seals. A GEL lubrication system - modified from the one described in section 5.4 - will then supply low and high-pressure GELs and other fluids to the seals tested.

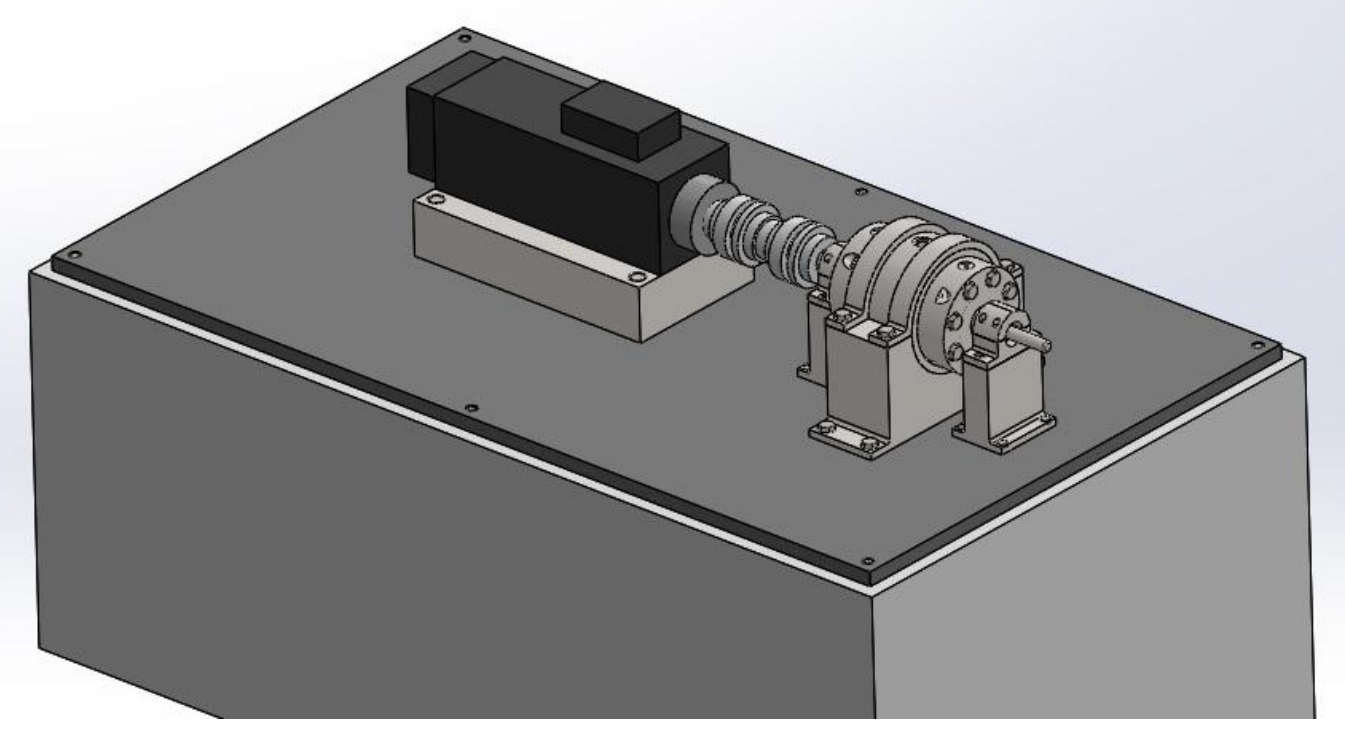

Figure 6.1. A 3D solid model depiction of the GEL Seal Test Rig test section. 
The test rig motor (Figure 6.2) is a Giordano Colombo RS 135 spindle motor, equipped with a variable frequency drive and designed for speeds up to $15,000 \mathrm{rpm}$ at 18 hp. These power and speed range specifications are based upon the seal performance predictions described in section 6.2. The motor is connected to the test rig rotor via a single-flex coupling-torque transducer-single-flex coupling arrangement (Interface) that will allow for torque measurements and provide necessary flexure for any slight misalignment of the rotor and motor that might take place, though final alignment procedures carried out after assembly should prevent this from occurring.

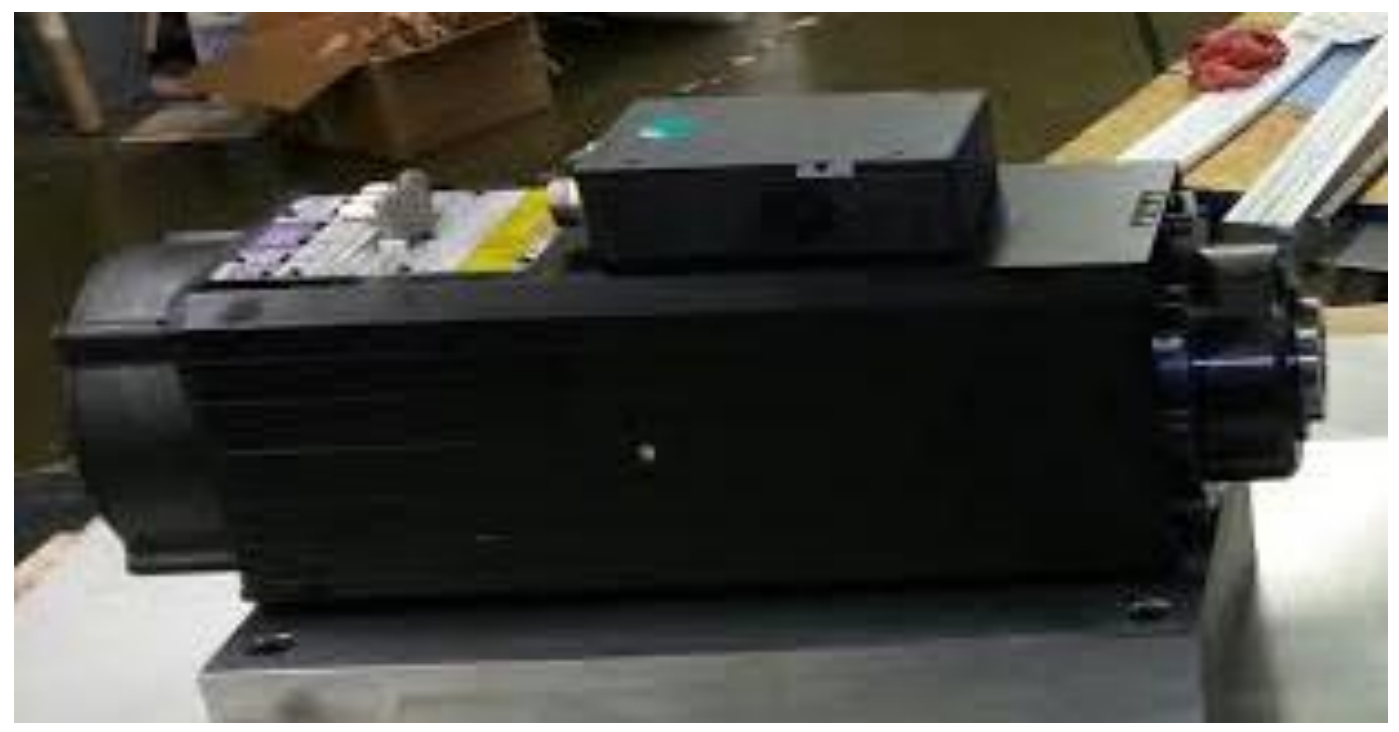

Figure 6.2. The Giordano Colombo spindle motor driving the GEL seal test rig.

The seal rig rotor (Figure 6.3) is 20" in total length, with 5" lengths at each axial end having a diameter of $20 \mathrm{~mm}$. The central section is 10 " in length with a 2 " diameter. This larger diameter will allow for greater surface speeds in the seal test section. The rotor is constructed of 17-4 stainless steel and will be coated with chrome in the test section to 
prevent galling of the rotor or seals should a misalignment-induced contact occur. Details of a rotordynamic analysis performed for the test rig rotor are discussed in section 6.3 .

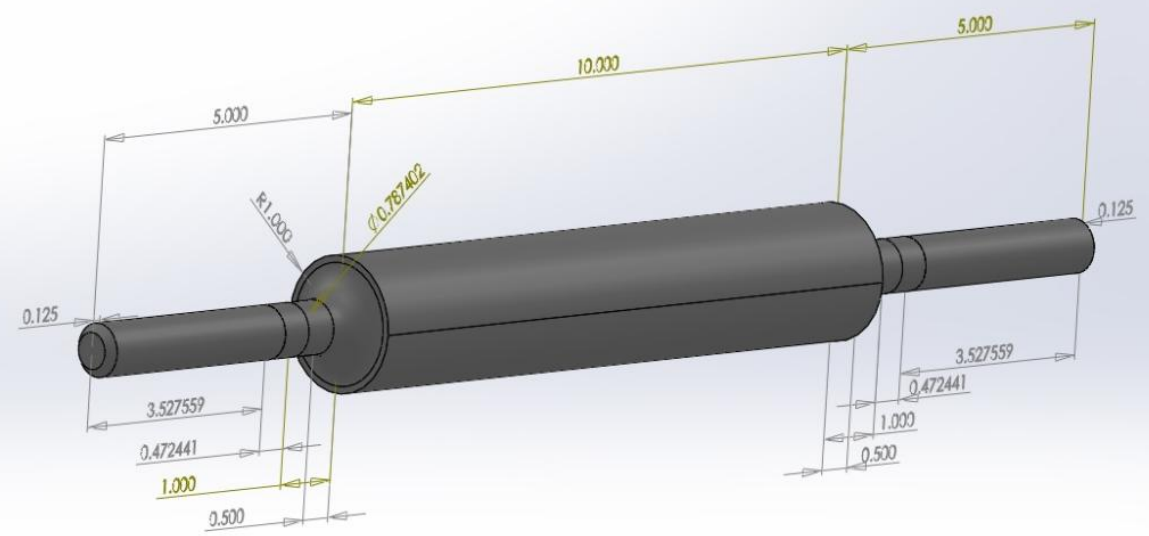

Figure 6.3. The GEL Seal Test Rig rotor.

The rotor is supported by two high-speed ball bearings (SKF 6004 Series) with a 20 $\mathrm{mm}$ bore, $42 \mathrm{~mm}$ outer diameter, and $12 \mathrm{~mm}$ axial length. These bearings have an 828 hour life and can support dynamic loads of up to $650 \mathrm{lbf}$. The bearings will be housed in custom split-design housings.

The test section (Figure 6.4) consists of a central housing with seals bolted onto each axial end. The test section is constructed of a high-strength 15-5 precipitation-hardened stainless steel. The design of the seals and analysis of the test section will be discussed further in sections 6.2 and 6.4. Fluid from the supply system will enter the test section through an SAE fitting machined into the top of the central housing. After traveling through the seals the fluid will then enter an expansion chamber, allowing for a significant pressure drop across the seal. Single-tooth labyrinth seals will prevent leakage 
from the axial ends of the seal cartridges while low-back pressure outlets at the bottom of the expansion chambers will collect the fluid and route it back into the supply system. As discussed further in section 6.2, this design will allow for multiple seal designs to be tested due to the interchangeable nature of the seal cartridges.

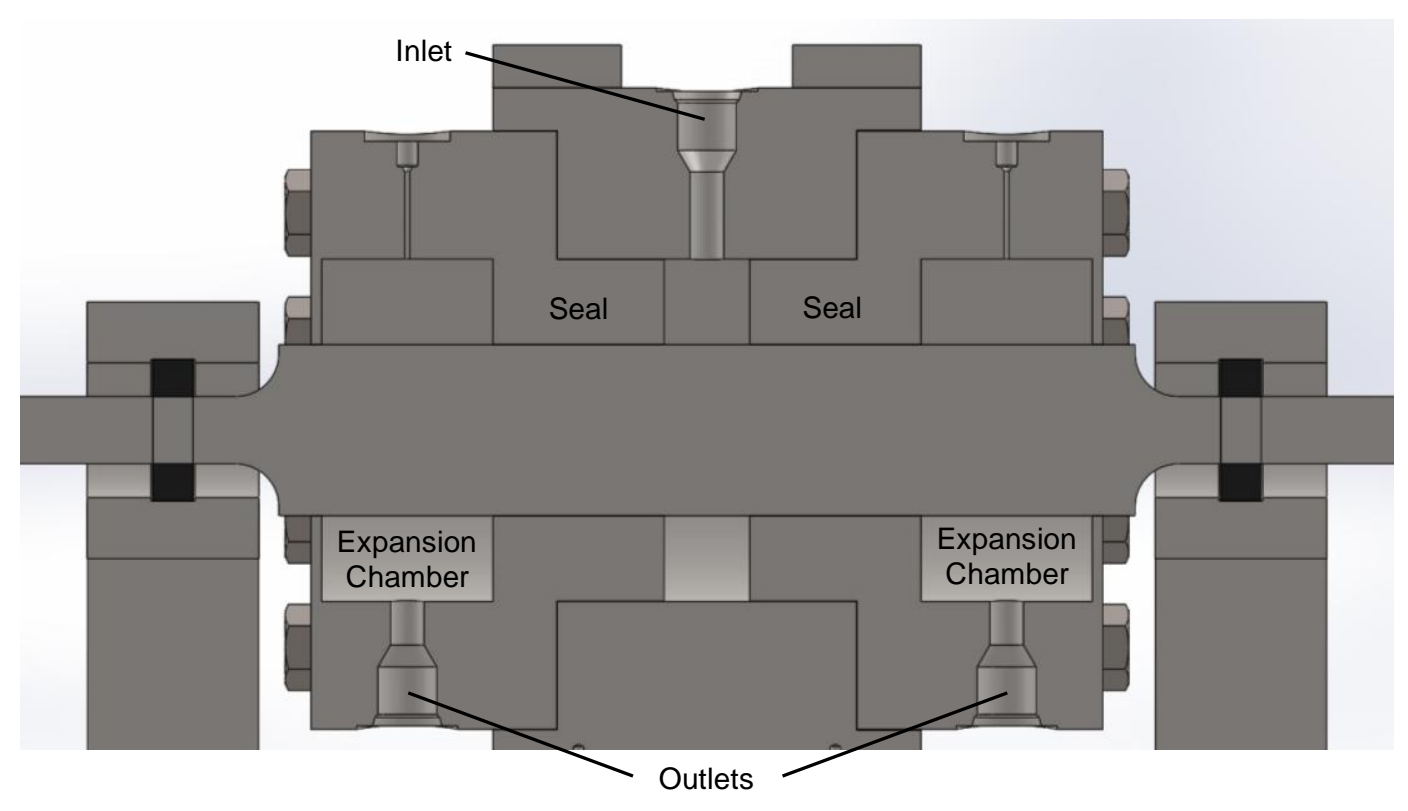

Figure 6.4. Cross-sectional view of the seal rig test section.

\subsection{Seal Design and Performance Predictions}

For the initial set of experiments to be performed with the test rig, two commonly used high-pressure annular seals were designed for performance testing. These designs include a plain, smooth-surface annular seal and a hole pattern seal. The plain annular seal, depicted in Figure 6.4, is 2 " in axial length with a radial clearance of 0.001 ". The expansion chamber is also 2 " in axial length with a 1 " radial clearance to allow for expansion of the leaked fluid as the pressure is reduced at the seal outlet. The single-tooth 
end seal has a length of 0.125 " with a radial clearance of 0.001 ". In addition to the SAE fittings on the bottoms of the seals used to collect the leakage, the seals will also have five high-pressure ports machined into them for pressure measurements in the expansion chamber as well as pressure and temperature measurements at two locations along each seal face (Figures 6.4 and 6.5). Figure 6.5 shows how two of the four ports are connected to the seal face, with a small hole showing the location of the other two connector holes in the axial direction. The holes are spaced at $1 / 3$ and $2 / 3$ of the total axial length of the seals.

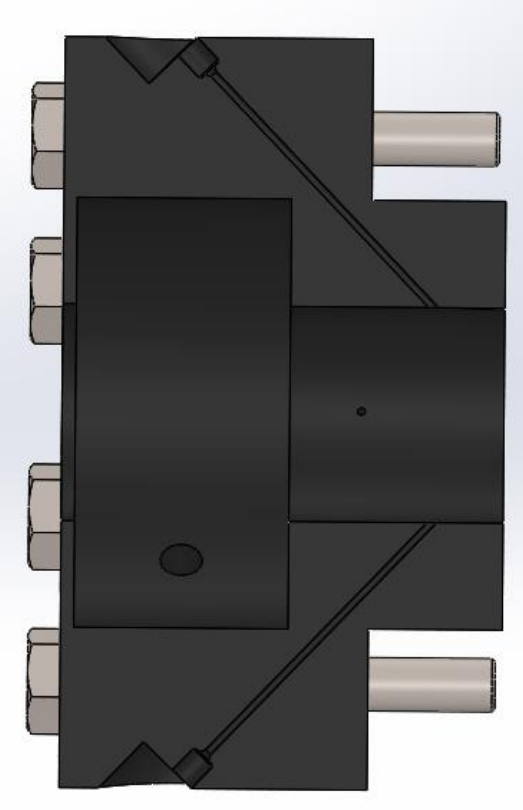

Figure 6.5. High-pressure ports machined into the seal cartridges for data collection. 
The hole pattern seal cartridges have the same exact dimensions as the plain annular seals, but with the addition of the hole pattern on the seal face. Hole pattern seals have been found to reduce leakage and destabilizing cross-coupled stiffness forces in gas seals $[124,135,140,164,166,169]$, therefore it will be interesting to see how they compare to plain annular seals in a liquid or GEL environment, particularly as the gas phase separates from the GEL as the pressure drops across the seal face. The hole geometry includes a 0.125 " outer diameter, a 0.125 " depth, and a 0.017 " material gap between the edges of each hole pair. These design parameters are based on a standard design reported by [143] for a hole pattern seal, resulting in a $70 \%$ hole coverage for the seal by surface area.

As the test rig was being designed, the expected performance of the seals needed to be known to ensure that many of the other test rig components (motor, rotor, seal clearance, pumping system, flow meters, etc.) were being sized correctly. To predict the performance of the test rig seals for these design purposes the computational fluid dynamics software package ANSYS CFX was used to analyze a scenario that would produce some of the highest leakage, power loss, and destabilizing rotordynamic characteristics of the seals and conditions expected to be studied with the test rig. This "worst-case" scenario involved modeling the plain annular seals as these seals are expected to produce the highest leakage rates of the seals analyzed. A large pressure gradient of 856 psi absolute was used to simulate a $20 \%$ carbon dioxide mass fraction in the lubricant which would also produce high leakage and significant destabilizing crosscoupled stiffness forces. A single-phase liquid was assumed in the modeling approach as well to both simplify the analysis and produce some of the largest of the destabilizing 
stiffness forces expected. A 10,000 rpm rotating speed was also used to maximize leakage, power loss, and cross-coupled stiffness values.

The leakage results of this simulation produced a value of approximately 1 gpm per seal. This result revealed that the GEL supply system was properly sized to deliver enough fluid to the test section, resulting in measured leakage rates that will not be limited by the capacity of the supply system. The power loss of the seal was estimated at approximately $6 \mathrm{hp}$. Accounting for two seals as well as a factor of safety, the $18 \mathrm{hp}$ spindle motor was chosen for the seal test rig. The synchronously reduced stiffness and damping coefficients (Figure 6.6) produced large values of stiffness due to the small clearance, high speed, incompressible fluid, and large pressure drop chosen. Of particular concern were the extremely large cross-coupled stiffness terms, though it is emphasized that these are likely much higher than that of an actual GEL due to the multi-phase nature of a GEL expanding across a seal face. However, these coefficients were useful as they could be considered to be a worst-case scenario for the rotordynamic analysis discussed in the following section.

$$
\begin{array}{ll}
\mathrm{K}_{\mathrm{xx}}=42,754 \mathrm{lb} / \mathrm{in} & \mathrm{C}_{\mathrm{xx}}=1,298 \mathrm{lb} \cdot \mathrm{s} / \mathrm{in} \\
\mathrm{K}_{\mathrm{xy}}=2,926,455 \mathrm{lb} / \mathrm{in} & \mathrm{C}_{\mathrm{xy}}=-330 \mathrm{lb} \cdot \mathrm{s} / \mathrm{in} \\
\mathrm{K}_{\mathrm{yx}}=-2,926,455 \mathrm{lb} / \mathrm{in} & \mathrm{C}_{\mathrm{yx}}=330 \mathrm{lb} \cdot \mathrm{s} / \mathrm{in} \\
\mathrm{K}_{\mathrm{yy}}=42,754 \mathrm{lb} / \mathrm{in} & \mathrm{C}_{\mathrm{yy}}=1,298 \mathrm{lb} \cdot \mathrm{s} / \mathrm{in}
\end{array}
$$

Figure 6.6. Stiffness and damping coefficients for the plain annular seal at 10,000 rpm. 


\subsection{Rotordynamic Analysis}

After completing the seal design analysis, a rotordynamic analysis of the seal test rig was performed to ensure its proper design and performance for the operating speeds considered. The test rig was designed in a manner that would hopefully result in the first critical speed being encountered above the operating range of the machine. By doing this any excessive vibration or rubbing in the seal areas could be avoided. The design parameters that resulted in raising this first critical speed include a larger shaft diameter and the use of highly stiff ball bearings. Locating the ball bearings as close to the center of the shaft as possible also resulted in a higher first critical speed.

In beginning the rotordynamic analysis, a series of simulations were first carried out to pinpoint the location of the first critical speed while neglecting seal effects as well as to verify that the rotordynamic finite element model used was built correctly. To do this, the analysis was performed using both the commercial ANSYS Mechanical finite element analysis software as well as the rotordynamic finite element analysis code described in section 3.2.3. The results of these two analyses produced first critical speed values of 51,856 rpm and 48,954 rpm, respectively, showing that the design approach was effective in raising the location of the first critical speed. A relative error of $5.6 \%$ between the two methods also produced confidence that the analysis was performed correctly.

The second step of the rotordynamic analysis was to include the effects of the seals to evaluate their effect on the location of the first critical speed. To do this, the seal coefficients produced from the CFD analysis described in section 6.2 were added to the rotordynamic finite element model. A second CFD analysis was also performed at higher 
speed to produce another set of seal coefficients. The results of the two analyses were then fit using linear interpolation so that the stability analysis could be performed at multiple speeds as the speed analyzed can affect the vibration frequencies predicted.

The results of this analysis are shown with a Campbell diagram in Figure 6.7. These results show how the worst-case scenario seal coefficients reduce the location of the first critical speed to approximately $12,000 \mathrm{rpm}$. While it is likely that the effects of the seals will not be this severe, it is important to know what their potential effects could be under the harshest of operating conditions. It is because of these results that initial experiments will likely only be performed at speeds up to $10,000 \mathrm{rpm}$. Though the rotordynamic performance of the test rig will depend on a number of factors including the fluid analyzed, pressure drop, seal design, and speed, great care must be taken when operating the test rig with high pressure liquids, especially at higher operating speeds. However, future modeling analyses that are planned to coincide with test rig experiments should aid in further predicting the rotordynamic performance of each set of experiments. This will allow for the safe operation of the test rig during all of the experiments performed. 


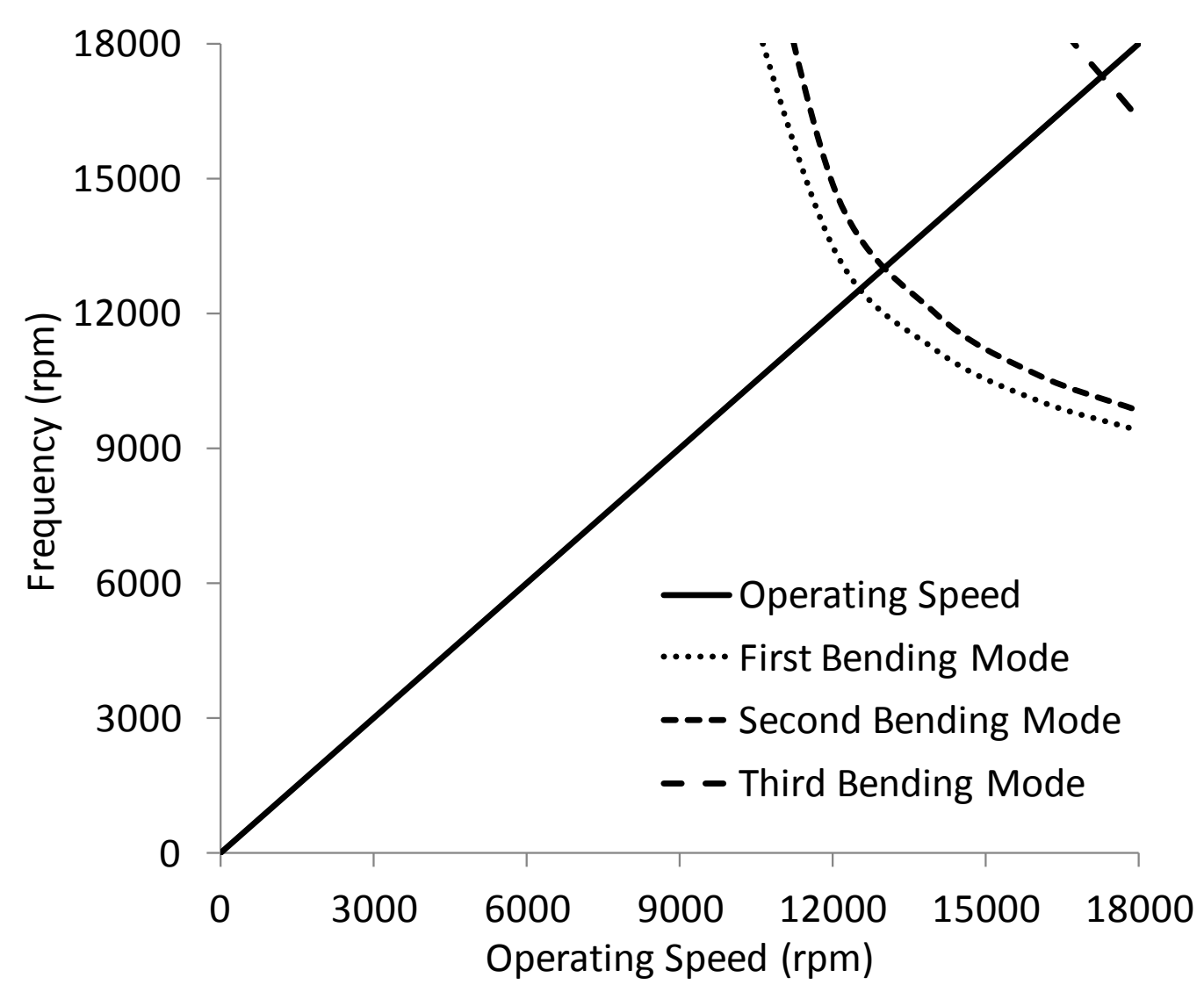

Figure 6.7. Campbell diagram of the frequency response of the GEL seal test rig.

\subsection{Test Section Finite Element Analysis}

To ensure that the test rig was properly designed for the design pressure of 1,500 psi, a finite element analysis of the test section was carried out using the ANSYS Mechanical commercial software. The finite element model of the test section is shown in Figure 6.8. To simulate the pressurized test section, a 1,500 psi pressure was applied to all of the inner surfaces of the test section. The pressure drop across the seals was then modeled by applying a pressure drop across the seal from 1,500 psi to atmospheric pressure. Bolt pretentions were applied to the axial connecting bolts in accordance with [117]. Gravity forces were applied to the entire model as well. Boundary conditions included fixed 
displacements along the bottom of the support structure, bonded contact surfaces between the bolt heads/threads and contacting surfaces, and sliding friction contact surfaces between the central housing and the seals. The materials assumed for the analysis include a 316 stainless steel for the housing and seals along with Grade 8 bolts.

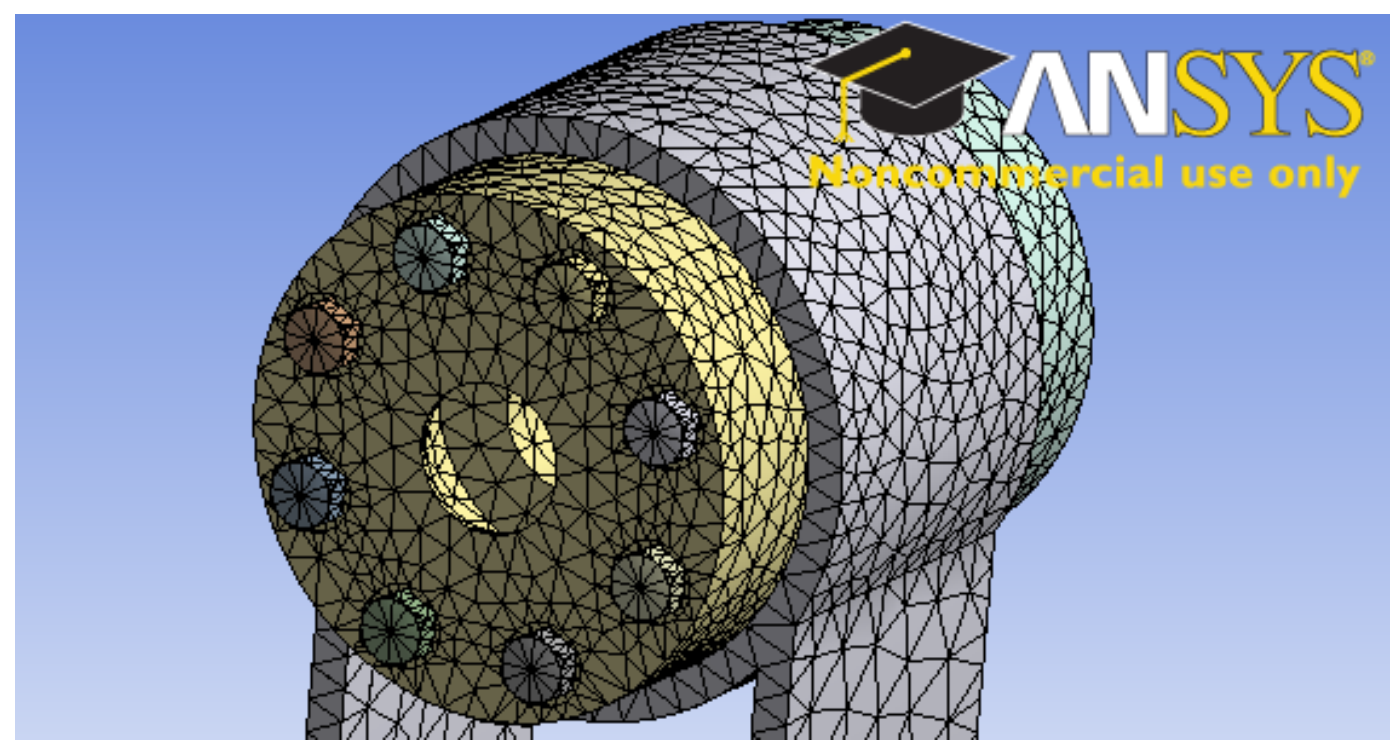

Figure 6.8. Finite element model of the GEL seal test rig test section.

The peak stresses in the test rig were found to occur in the bolts connecting the seals to the central housing (Figure 6.9). These stresses peaked at values near 85,000 psi generally, though the model predicted higher values at select elements on the bolts, most likely due to the mesh used. These results were sufficient to conclude that the highstrength Grade 8 bolts selected for the test rig would be adequate for the design pressure. Bolt fatigue calculations were also performed, ensuring that the bolts selected will last for at least 11,000 cycles. 


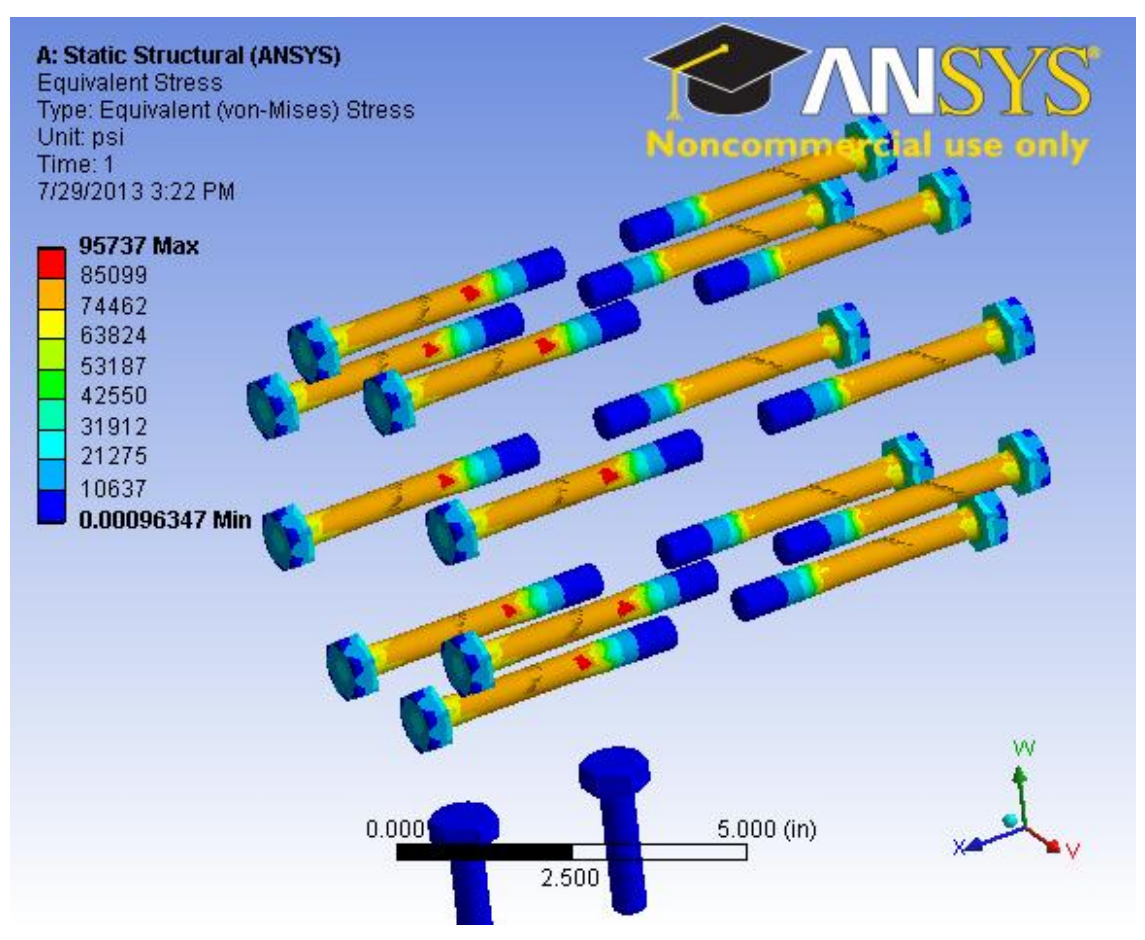

Figure 6.9. Peak stresses in the test rig bolts.

Peak stresses in the central housing were found to occur in the bolted joints (Figure 6.10). Stresses in these joints were found to reach their maximum at approximately 54,000 psi. The peak stresses in the seals were also found to occur in the bolted joints underneath the bolt heads (Figure 6.11), with maximum values also reaching approximately 54,000 psi. This led to the conclusion that a standard 316 stainless steel would not suffice as its yield strength is approximately 30,000-35,000 psi, thus a highstrength 15-5 precipitation-hardened stainless steel was selected as the housing and seal material to ensure both proper material strength and resistance to corrosion in the carbon dioxide environment. Similar to the results of the bearing test rig finite element analysis, the peak deformations and separations were also predicted to be very small, though the use of PTFE gaskets will help to ensure no leakage between the bolted joints. 


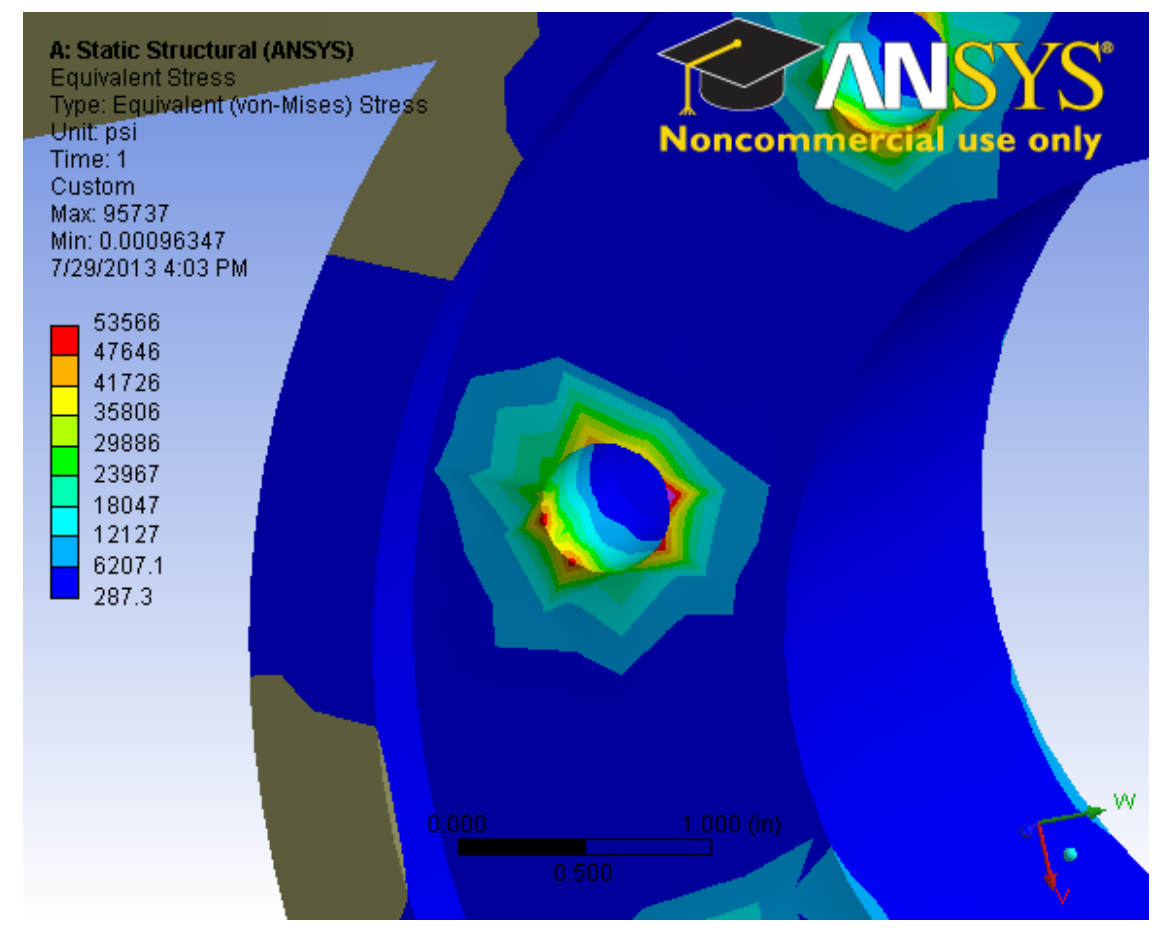

Figure 6.10. Peak stresses in the central housing.

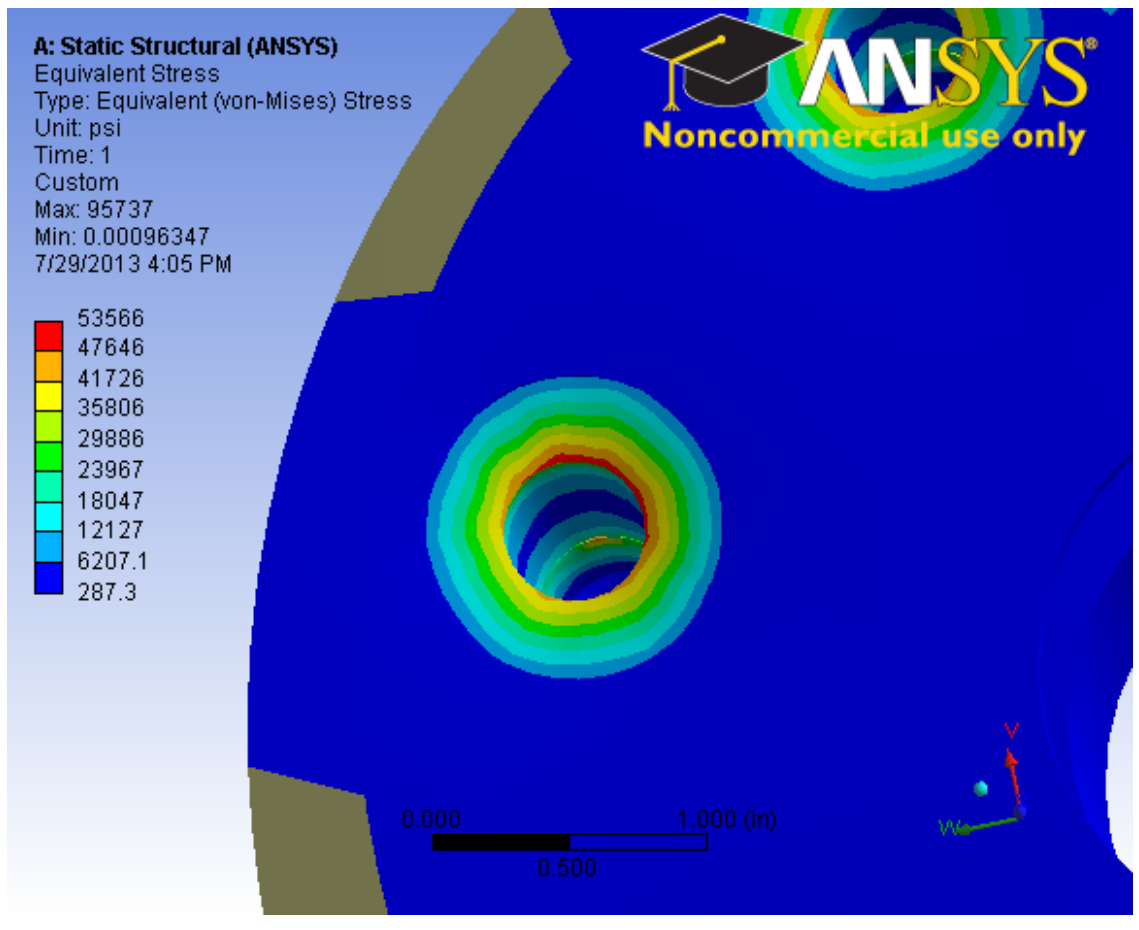

Figure 6.11. Peak stresses in the seal. 


\subsection{Base Modal Analysis}

A modal analysis of the test rig base and support structures was also performed to ensure that no structural vibration modes would be encountered in the operating speed range considered. After importing the 3D solid model to ANSYS Mechanical, the concrete base and carbon steel plate and stand materials were entered and the densities of the supporting stands were modified to account for the weight of the test section. The results of the analysis are shown in Figure 6.12. These results confirmed that the first structural mode was outside of the operating speed range ( $609 \mathrm{~Hz}$ or $36,563 \mathrm{rpm})$. This was largely due to the high stiffness properties of the concrete base.

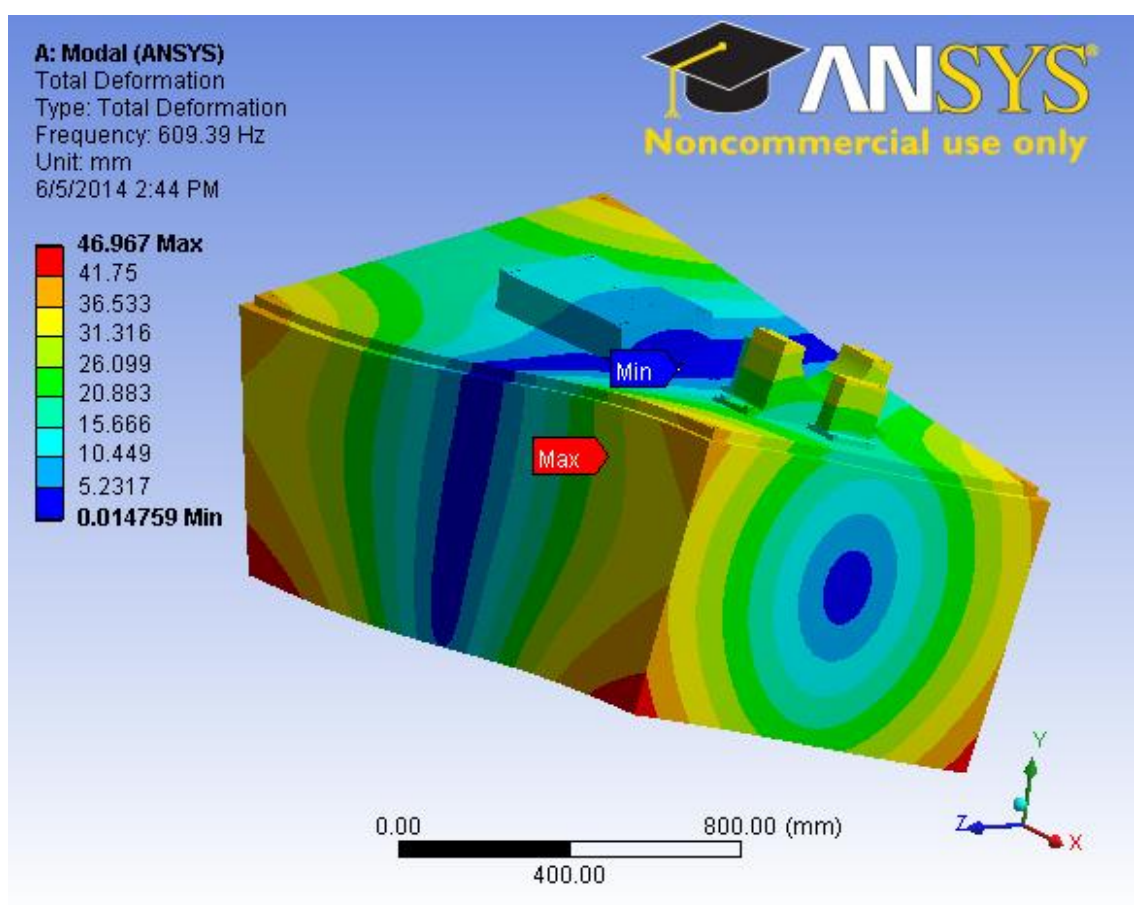

Figure 6.12. Modal analysis of the test rig base and support structures. 


\subsection{Lubrication System Modifications}

The GEL delivery system that will supply pure oil, GELs, and other fluids to the test section of the seal rig is the same lubricant supply system described in section 5.4. However, as that system was designed to provide back-pressure to the entire test section, modifications had to be made to the supply system in order for it to supply high-pressure fluids only to the inlet of the test section while providing as little back-pressure as possible to the outlets of the seals. This will then allow for a dramatic drop in pressure across the seal face and adequate collection of the seal leakage from the outlet ports in the seals.

The modified GEL supply system (Figure 6.13) includes the addition of a bypass line that is connected to both the test section inlet and the adjustable cartridge relief valves for providing back-pressure to the inlet. An additional pressure gauge and flow meter on this line will allow for monitoring of the inlet pressure as well as the precise calculation of fluid leakage through the seals in the test section. The outlets of the seals are then directly connected to the drainage line on the low-pressure side of the relief valve manifold via a tee fitting which will minimize back-pressure to the seal outlets while still allowing for further degassing, filtering, and temperature control of the oil circulating in the flow loop. 


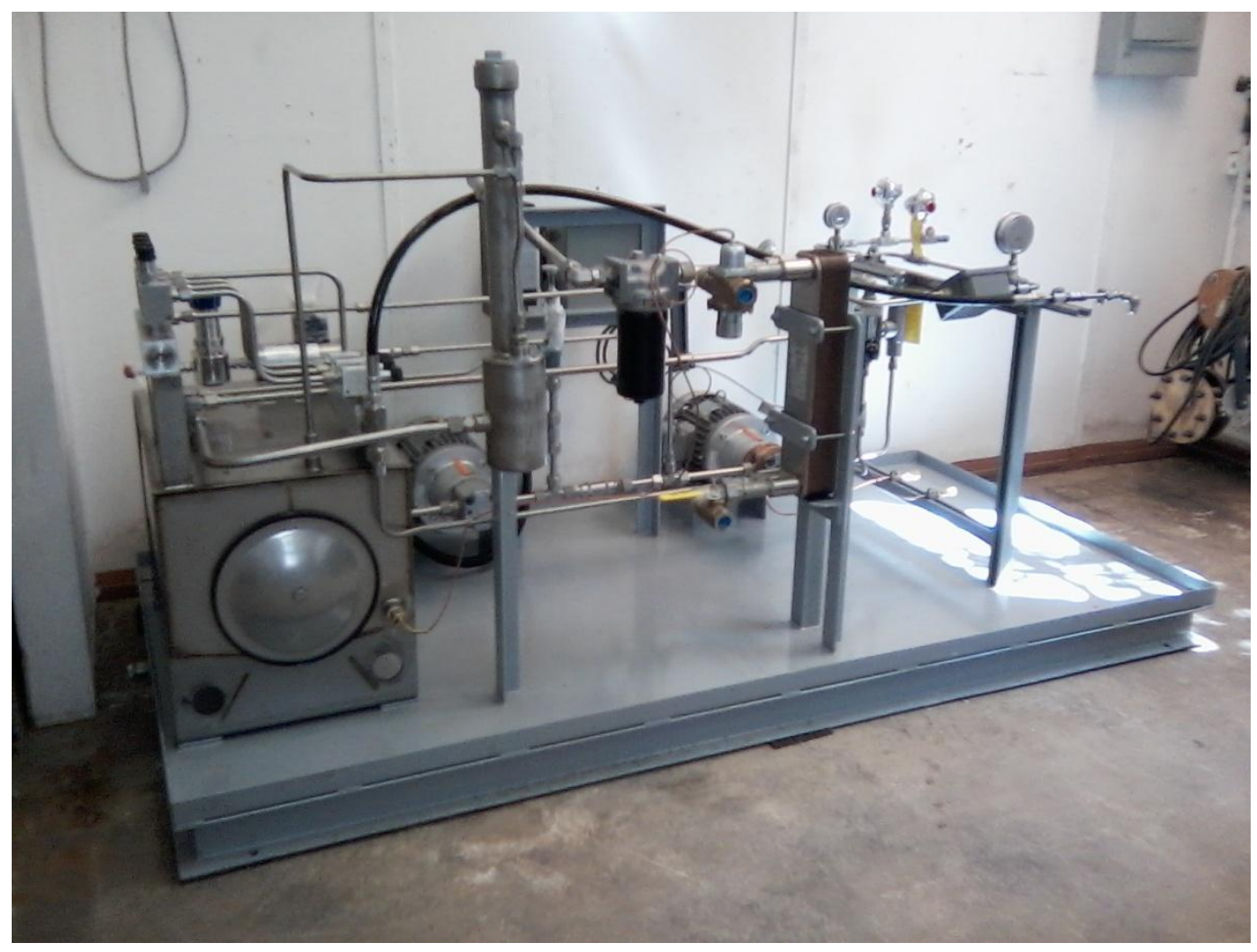

Figure 6.13. The modified GEL supply system for the GEL Seal Test Rig.

\subsection{Data Acquisition and Instrumentation}

The data acquisition system for the GEL Seal Test Rig will be similar to that of the system designed for the bearing test rig (section 5.5). The main acquisition system will consist of an 8-slot chassis supplied by National Instruments, a series of input and output voltage and amperage modules for receiving input signals and sending control signals to equipment, and a computer equipped with the LabView data acquisition and control software package.

In addition to pressure and temperature gauges and measurements logged at various stages of the GEL supply system, the test section will be equipped with six pressure transducers (Omega Engineering) and four K-type thermocouples (Omega Engineering) 
for measuring pressure and temperature distributions along the seal faces as well as pressures in the seal outlet areas. Two flow meters (Hedland H600 Series) in the fluid supply system will allow for the calculation of seal leakage in the test section. The power loss in the test section will be calculated by multiplying the operating speed by the measured torque of the system. Torque will be measured with a torque transducer (Interface T4 Series) positioned between the motor and rotor couplings with an expected torque range of up to $30 \mathrm{~N} \cdot \mathrm{m}$. This value was determined using model predictions and an adequate factor of safety. The LabView-equipped computer will then collect and store all of this information while providing output control signals to the carbon dioxide mass flow controller, the first-stage gear pump variable frequency drive, and the variable frequency drive of the test rig motor.

\subsection{Summary}

A Gas-Expanded Lubricants Seal Test Rig has been designed and assembly is currently underway. This test rig includes a versatile test section capable of testing many interchangeable seals under a variety of pressure conditions and with a variety of liquid, gas, and multi-phase fluids. All of the ancillary components for the system have been specified, received, and installation will be completed in the near future following modifications to the test facility. A number of performance, finite element, and rotordynamic analyses have been performed to ensure its proper design and operation. It is hoped that this unique test rig will not only allow users to continue answering important questions surrounding the use of high-pressure seals with gas-expanded 
lubricants, but that it will also serve as a useful platform and validation tool for years to come in the general experimental research of liquid, gas, and multi-phase seals. 


\section{Chapter 7}

\section{Conclusions and Future Work}

\subsection{Summary and Conclusions}

A novel lubrication technology in the form of gas-expanded lubricants has been proposed and studied in detail. Previous knowledge of standard and emerging technologies has been reviewed and the limitations of these technologies have been outlined. Gas-expanded lubricants stand to provide benefits that none of these other technologies offer including increased efficiency, reliability, and control without comprising other important machine lubrication considerations such as load capacity.

The first important contribution of this work has been to build upon previous knowledge of the properties of lubricant-carbon dioxide mixtures. A library of viscosity data has been created along with new knowledge on the diffusivity, thermal conductivity, and thermo-oxidative stability of these mixtures. This new knowledge then allowed for the accurate prediction of the performance of these fluids in numerous bearing designs and machine applications. It was concluded that in many applications these lubricants 
significantly increase bearing efficiency, reliability, and have the potential to provide for real-time control over machine rotordynamic stability. Effects on the peak displacements of unbalanced machine rotors were found to be minor, though when altered in combination with other bearing design parameters the lubricant properties were found to affect peak displacements in a significant enough manner to remove a rub impact from a system encountering a severe rub due to a large unbalance. These new approaches to improving machine performance could be very beneficial to an industry that is constantly striving to build more efficient and harder driven machines for energy production and resource transport.

Another important contribution of this work is the development of designs compatible with these moderate-pressure, tunable lubricants. New bearing housings and lubrication systems have been designed and a fully controllable lubrication system has been received for use with a versatile seal test rig that will be used to study GEL-compatible seals as well as a variety of other high-pressure liquid, gas, and multi-phase seals. Overall, this body of knowledge will build the foundation for future commercial development work that will deliver this novel lubrication technology to the turbomachinery industry.

\subsection{Future Work}

As with any technological development work, there are always more questions to ask, alternative paths to consider, and desires to further improve the performance of that technology. The following sections offer suggestions and ideas for future work that could be performed to answer new questions surrounding the use of gas-expanded lubricants. These ideas will also look to explore alternative designs that may improve the 
performance of these fluids or make them more feasibly implemented in real-world applications. They will also call for the need to begin collaborating with the industry this technology is targeting in order to be able to answer important design and applicationbased questions that are too difficult to assess in a comprehensive manner in a purely academic setting. It is the hope that this future work will then lead to the development of the first prototypes of this potentially groundbreaking technology.

\subsubsection{Seal Testing and Model Validation}

The first recommendation for future work is to complete the assembly of the GEL Seal Test Rig. At the time of this writing all of the components for this test rig have been received, with only facilities work remaining as the final hurdle to allowing experiments to commence. A great deal of work and resources have been put into this test rig and it will serve many future students well as they study a variety of seal designs for various machine applications.

The GEL Seal Test Rig will provide a plethora of new information to academic researchers and industry designers alike. From the perspective of further developing the GEL technology, the knowledge gained from this test rig will determine the types of seals that will be compatible with GELs, as well as what types of seals will be the most appropriate for various sets of application needs and constraints. This knowledge can then lead to the confident design of machines that are fully compatible with the GEL technology as well as easier identification of applications where this technology would be the most beneficial.

From the perspective of designing better seals for the turbomachinery industry, this test rig will allow for the successful experimental testing of a wide range of seal designs 
more commonly used by this industry. These seal designs include both low-pressure and high-pressure seals, as well as those used with pure liquids, pure gases, and multi-phase mixtures. As increasingly powerful predictive analysis and design tools are being developed for the industry, it will be vital that these tools are validated for a wide range of designs and operating conditions.

This test rig has a lot of potential for improvement as well. Though it currently has the ability to provide important validation and performance information on pressure/temperature distributions, power loss, and leakage, other instrumentation could be added to the test rig to increase its value as well. These enhanced capabilities could include the ability the measure friction factors that are important to widely used bulk flow analysis tools, as well as the ability to experimentally measure seal stiffness and damping coefficients that are extremely important for machine rotordynamic analyses and analysis code validation.

\subsubsection{Alternative Design Analysis}

Throughout this work, one of the most fundamental assumptions that has been made about the GEL technology is that a fully flooded lubrication approach must be used to ensure that this technology could be feasibly implemented in a manner that did not deliver complex behaving, multi-phase fluids to the support region of the bearing. While at a first glance a fully flooded delivery would be the most reliable way of ensuring this successful implementation, it is also believed that alternative approaches to GEL delivery in bearings should be considered.

Two of the leading alternatives to flooded-lubrication bearings in use by the industry today include directed lubrication designs (including leading-edge groove pads and spray 
bar blockers) and hydrostatic bearings. The benefit of directed lubrication is that the fluid is only delivered to the fluid film region of the bearing, further reducing both bearing power loss and hot oil carryover while maintaining adequate support for the shaft, though this technology comes with no tunable control over the bearing performance. The benefits of hydrostatic bearings include being able to use process gases as lubricants as well as having the capability of supporting heavy loads under low-speed conditions due to the large hydrostatic pressures of the supply fluid while allowing the fluid to expand upon leaving the support region, thus not requiring the use of high-pressure bearing seals. This technique, however, is not used for the real-time control of bearing and rotordynamics either.

One alternative approach to the flooded delivery of GELs in bearings would be to utilize the design and benefits of both of these established approaches to bearing lubrication. If one could deliver a pressurized, single-phase GEL directly to the fluid film region, this would still allow for the use of a tunable lubricant in this most important region of the bearing while eliminating the need for a pressurized bearing housing and high-pressure seals. The fact that the fluid film region is the location in the bearing with the highest pressures would help to ensure a single-phase fluid, and allowing the expansion of the fluid in the divergent region of the bearing would also provide additional cooling in what is often the warmest location of the bearing. The challenge would then be to adequately collect the separated carbon dioxide in the system for recompression and reuse so that large amounts of carbon dioxide would not be required for this approach. However, it is likely that these separation and collection technologies already exist for use in enhanced oil recovery and other applications of similar nature. 
It is therefore proposed that future work include the study of this alternative GEL lubrication approach. The use of a combined computational fluid dynamics-finite element analysis approach in commercial software available such as ANSYS will allow for a thorough analysis of this approach, taking into account the fluid phase behavior, diffusion/mixing, and thermal expansion of the carbon dioxide while allowing for the calculation of effects on bearing power loss, operating temperature, and the potential control over bearing stiffness and damping coefficients. If the results are promising, this approach could then be proposed as one that could easily be implemented using a combination of already established bearing designs and lubrication technologies.

\subsubsection{Systems Analysis}

Up until this point, gas-expanded lubricants have been studied for their potential effects on bearing and rotordynamic performance, and so far the results have been promising. However, due to limited data in the academic literature it has been difficult to assess some of the other important aspects of implementing the technology. These other considerations include implications for the design of bearings, bearing housings, seals, lubrication systems, and other components in real machines used for very specific applications. These design implications then have direct impacts on other factors that need to be considered such as operational control, machine performance, and the cost of implementation. As these factors are likely the determining factors as to whether or not this technology will ever actually be implemented, it is of upmost importance that they be considered in future system-level analyses.

For example, it has been shown that the effects of lubricant properties on bearing efficiency can be quite significant, with a $40 \%$ reduction in bearing losses found with the 
use of GELs in the centrifugal compressor discussed in section 3.3. However, this raises the question of how these efficiency gains in the bearings compare to the efficiency of the entire machine. Due to a lack of information on the power usage of the machine, the types of lubrication systems used or needed for normal and GEL-lubricated conditions, and the types of bearing seals required for each lubrication condition, this becomes a difficult parameter to quantify. Under best-case conditions, if one were to assume that changes in lubrication system power usage and seal power loss were negligible, and that the power consumed by the compressor was in the 5-10 MW range based on the rotor size and number of stages, the $5.58 \mathrm{~kW}$ gain in total bearing efficiency would result in a machine-level increase in efficiency of $0.05-0.11 \%$. While this result is promising as even small efficiency gains can result in valuable long-term returns in cost savings, under a worst-case condition one could argue that the high-pressure oil pumps and oil seals required for the GEL technology would likely result in a net loss in efficiency for the machine. Again, these scenarios are extremely difficult to quantify, but this example highlights the importance of having detailed information on the designs of the machines being considered for this technology in being able to quantify both the costs and benefits associated with its use.

Because of these important considerations, it is proposed that future system-level analyses be performed that are more comprehensive in nature, considering all of the design, performance, and economic factors listed above. However, due to the limited availability of the information required to perform such an analysis, it is proposed that this work be carried out in collaboration with interested parties in the industries in which this technology could be useful. Only by sharing important design, application, and cost 
information between researchers and industry designers and end users will an analysis of this type be able to be performed in a comprehensive manner that will result in the identification of feasible applications in which this technology can be successfully implemented. Analyses of this type could then result in the rapid development and use of the GEL technology in the applications and industries that have been identified as targets for its beneficial use.

\subsubsection{Prototype Development}

No new technology can be trusted for use by the general public until adequate proofof-concept work has been carried out. It is therefore proposed that as a final stage of the future work proposed that a fully controllable, GEL-lubricated prototype be developed and operated to demonstrate the full capabilities of this technology to the private sector. This prototype would be developed upon completing the preceding analyses described in this chapter and would stand to benefit greatly from their completion. Should it be determined that a flooded-lubrication design would be the most feasible form of delivering GELs to bearings in a controllable manner, the results of the GEL seal testing will be vital to the successful development of a prototype. However, should an alternative design such as the one proposed in section 7.2.2 be deemed the most feasible for implementation of the GEL technology, the information from that analysis will then serve as the basis for providing the first demonstration of that approach. The systems analysis will also be extremely important to determining viable applications for the GEL technology and will influence the design of the initial prototype. This demonstration will then not only provide a technical proof-of-concept to designers and end users, but it will also demonstrate how to implement the technology in a manner that is both compatible 
with existing machinery and in a manner that is cost effective. Overall, it is the hope that all of the future work proposed will further aid the academic and industrial communities in realizing the full potential of the gas-expanded lubricant technology. 


\section{References}

[1] Angantyr, A., and Aidanpaa, J., 2006, "Constrained Optimization of Gas Turbine Tilting Pad Bearing Designs," Journal of Engineering for Gas Turbines and Power, 128(4), pp. 873-878.

[2] Brechting, B., Flack, R., and Cloud, H., 2005, "Influence of Journal Speed and Load on the Static Operating Characteristics of a Tilting-Pad Journal Bearing with Ball-and-Socket Pivots," Tribology Transactions, 48(3), pp. 283-288.

[3] Nicholas, J. C., 1977, "A Finite Element Dynamic Analysis of Pressure Dam and Tilting Pad Bearings," Ph.D. Thesis, University of Virginia, Charlottesville, VA.

[4] Michaelis, K., Höhn, B., and Hinterstoißer, M., 2011, "Influence Factors on Gearbox Power Loss," Industrial Lubrication \& Tribology, 63(1), pp. 46-55.

[5] Stachowiak, G.W., and Batchelor, A.W., 2005, "Engineering Tribology," Elsevier Butterworth-Heinemann.

[6] Hauk, A., and Weidner, E., 2000, "Thermodynamic and Fluid-Dynamic Properties of Carbon Dioxide with Different Lubricants in Cooling Circuits for Automobile Application," Industrial \& Engineering Chemistry Research, 39(12), pp. 46464651.

[7] Khonsari, M.M., and Booser, E.R., 2001, "Applied Tribology: Bearing Design and Lubrication," John Wiley \& Sons. 
[8] Lihua, Y., Shemiao, Q., and Lie, Y., 2009, "Analysis on Dynamic Performance of Hydrodynamic Tilting-Pad Gas Bearings using Partial Derivative Method," Journal of Tribology, 131(1), pp. 011703.

[9] Muszýnska, A., 2005, "Rotordynamics," CRC.

[10] Glavatskih, S., Fillon, M., and Larsson, R., 2002, "The Significance of Oil Thermal Properties on the Performance of a Tilting-Pad Thrust Bearing," Journal of Tribology, 124(2), pp. 377-385.

[11] Ripple, D. E., and Fuhrmann, J. F., 1989, "Performance Comparisons of Synthetic and Mineral Oil Crankcase Lubricant Base Stocks," Journal of Synthetic Lubrication, 6(3), pp. 209-232.

[12] Martins, R., Cardoso, N., and Seabra, J., 2008, "Gear Power Loss Performance of Biodegradable Low-Toxicity Ester-Based Oils," Proceedings of the Institution of Mechanical Engineers, Part J: Journal of Engineering Tribology, 222(3), pp. 431440.

[13] Harangozo, A. V., Stolarski, T. A., and Gozdawa, R. J., 1991, "The Effect of Different Lubrication Methods on the Performance of a Tilting-Pad Journal Bearing," Tribology Transactions, 34(4), pp. 529-536.

[14] Barrett, L., Gunter, E., and Allaire, P., 1978, "Optimum Bearing and Support Damping for Unbalance Response and Stability of Rotating Machinery," Journal for Engineering for Power, 100(1), pp. 89-94.

[15] Nicholas, J. C., and Kirk, R. G., 1979, "Selection and design of tilting pad and fixed lobe journal bearings for optimum turborotor dynamics," Proceedings of the 
Eight Turbomachinery Symposium, Texas A\&M University, College Station, TX, pp. 43-57.

[16] Nicholas, J., and Kirk, R., 1982, "Four Pad Tilting Pad Bearing Design and Application for Multistage Axial Compressors," Journal of Lubrication Technology, 104(4), pp. 523-529.

[17] Bhat, R., Rao, J., and Sankar, T., 1982, "Optimum Journal Bearing Parameters for Minimum Rotor Unbalance Response in Synchronous Whirl," Journal of Mechanical Design, 104(2), pp. 339-344.

[18] Untaroiu, C. D., and Untaroiu, A., 2010, "Constrained Design Optimization of Rotor-Tilting Pad Bearing Systems," Journal of Engineering for Gas Turbines and Power, 132(12), pp. 122502.

[19] San Andres, L. A., 1991, "Effects of Fluid Compressibility on the Dynamic Response of Hydrostatic Journal Bearings," Wear, 146(2), pp. 269-283.

[20] Allaire, P., Humphris, R., and Barrett, L., 1986, "Critical speeds and unbalance response of a flexible rotor in magnetic bearings," Proceedings of the European Turbomachinery Symposium.

[21] Allaire, P., Humphris, R., and Kasarda, M., 1987, "Magnetic Bearing/Damper Effects on Unbalance Response of Flexible Rotors," IECEC, pp. 824-828.

[22] Humphris, R., Allaire, P., and Lewis, D., 1988, "Design and Testing of Magnetic Bearings for Vibration Reduction," Cambridge University Press, pp. 92-100.

[23] Maslen, E., Hermann, P., Scott, M., 1989, "Practical Limits to the Performance of Magnetic Bearings: Peak Force, Slew Rate, and Displacement Sensitivity," Journal of Tribology, 111(2), pp. 331-336. 
[24] Williams, R. D., Keith, F. J., and Allaire, P. E., 1990, "Digital Control of Active Magnetic Bearings," Industrial Electronics, IEEE Transactions, 37(1), pp. 19-27.

[25] Knospe, C. R., Hope, R. W., Fedigan, S. J., 1995, "Experiments in the Control of Unbalance Response using Magnetic Bearings," Mechatronics, 5(4), pp. 385-400.

[26] Hu, T., Lin, Z., Jiang, W., 2005, "Constrained Control Design for Magnetic Bearing Systems," Journal of Dynamic Systems, Measurement, and Control, 127(4), pp. 601-616.

[27] Yoon, S. Y., Lin, Z., and Goyne, C., 2010, "Control of compressor surge with active magnetic bearings," Decision and Control (CDC), 2010 49th IEEE Conference, pp. 4323-4328.

[28] Dimond, T., Allaire, P., Mushi, S., 2012, "Modal Tilt/Translate Control and Stability of a Rigid Rotor with Gyroscopics on Active Magnetic Bearings," International Journal of Rotating Machinery.

[29] Mushi, S. E., Lin, Z., and Allaire, P. E., 2012, "Design, Construction, and Modeling of a Flexible Rotor Active Magnetic Bearing Test Rig," Mechatronics, IEEE/ASME Transactions, 17(6), pp. 1170-1182.

[30] Mahdavi, S., Mansouri, S., and Kimiaeifar, A., 2012, "Effects of using Smart Fluid in Lubrication on Skirt-Liner Friction," Industrial Lubrication and Tribology, 64(2), pp. 90-97.

[31] Pavlioglou, S., Mastrokalos, M., Papadopoulos, C., 2014, "Tribological Optimization of Thrust Bearings Operated with Lubricants of Spatially Varying Viscosity," Proceedings of the ASME Turbo Expo 2014: Turbine Technical 
Conference and Exposition, Paper No. GT2014-25292, June 16-20, 2014, Düsseldorf, Germany, GT2014, pp. 1-10.

[32] Hauk, A., 2001, "Thermo- Und Fluiddynamik Von Synthetischen Schmierstoffen Mit Kohlendioxid Als Kältemittel in PKW-Klimaanlagen," Ph.D. Thesis, Ruhr University, Bochum, Germany.

[33] Yokozeki, A., 2007, "Solubility Correlation and Phase Behaviors of Carbon Dioxide and Lubricant Oil Mixtures," Applied Energy, 84(2), pp. 159-175.

[34] Youbi-Idrissi, M., Bonjour, J., Terrier, M., Meunier, F., and Marvillet, C., 2003, "Solubility of $\mathrm{CO}_{2}$ in a Synthetic Oil," Proceedings of the International Congress of Refrigeration.

[35] Bobbo, S., Fedele, L., and Pernechele, F., 2008, "Solubility of CO2 in Commercial POE Oils with Different Standard Viscosity," International Refrigeration and Air Conditioning Conference, Paper 988.

[36] Clarens, A., Younan, A., Wang, S., and Allaire, P., 2010, "Feasibility of GasExpanded Lubricants for Increased Energy Efficiency in Tilting-Pad Journal Bearings," Journal of Tribology, 132(3), pp. 031802-1-0131802-8.

[37] Bair, S., 2004, "The High Pressure Rheology of Mixtures," Journal of TribologyTransactions of the ASME, 126(4), pp. 697.

[38] Liu, Y., Wang, Q. J., and Krupka, I., 2008, "The Shear-Thinning Elastohydrodynamic Film Thickness of a Two-Component Mixture," Journal of Tribology, 130(2), pp. 021502. 
[39] Wang, S., Al-Sharif, A., Rajagopal, K., and Szeri, A., 1993, "Lubrication with Binary Mixtures: Liquid-Liquid Emulsion in an EHL Conjunction," Journal of Tribology, 115(3), 515-522.

[40] Prata, A., Grando, F., Silva, A., 2004, "Improving Compressor Design Through Modeling of Phase Change and Two-Phase Flow Phenomena," International Compressor Engineering Conference.

[41] Grando, F., Priest, M., and Prata, A., 2006, "A Two-Phase Flow Approach to Cavitation Modelling in Journal Bearings," Tribology Letters, 21(3), pp. 233-244.

[42] Grando, F., Priest, M., and Prata, A., "Lubrication in Refrigeration Systems: Performance of Journal Bearings Lubricated with Oil and Refrigerant Mixtures," Tribology and Interface Engineering Series, 48, pp. 481-491.

[43] Younan, A., Cao, J., Dimond, T., and Allaire, P., 2010, "Nonlinear Analysis of Squeeze Film Damper with Entrained Air in Rotordynamic Systems," Tribology Transactions, 54(1), pp. 132-144.

[44] Taylor, R.I., Dixon, R.T., Wayne, F.D., and Gunsel, S., 2005, "Lubricants \& Energy Efficiency: Life -Cycle Analysis," Tribology and Interface Engineering Series, 48, pp. 565-572.

[45] Frene, J., Nicolas, D., Degueurce, B., and Berthe, D., 1997, "Hydrodynamic Lubrication: Bearings and Thrust Bearings," Elsevier Science.

[46] Booser, E., Missana, A., and Ryan, F., 1970, "Performance of Large Steam Turbine Journal Bearings," ASLE Transactions, 13(4), pp. 262-268.

[47] Anderson, W. J., and Ludwig, L. P., 1968, "Bearing and Seal Technology," NASA, 19690003251. 
[48] Matthews, M. A., Rodden, J. B., and Akgerman, A., 1987, "High-Temperature Diffusion of Hydrogen, Carbon Monoxide, and Carbon Dioxide in Liquid NHeptane, N-Dodecane, and N-Hexadecane," Journal of Chemical \& Engineering Data, 32(3), pp. 319-322.

[49] Wang, L.., Lang, Z.., and Guo, T., 1996, "Measurement and Correlation of the Diffusion Coefficients of Carbon Dioxide in Liquid Hydrocarbons Under Elevated Pressures," Fluid Phase Equilibria, 117(1-2), pp. 364-372.

[50] Zhang, Y. P., Hyndman, C. L., and Maini, B. B., 2000, "Measurement of Gas Diffusivity in Heavy Oils," Journal of Petroleum Science and Engineering, 25(12), pp. 37-47.

[51] Tharanivasan, A. K., Yang, C., and Gu, Y., 2004, "Comparison of Three Different Interface Mass Transfer Models used in the Experimental Measurement of Solvent Diffusivity in Heavy Oil," Journal of Petroleum Science and Engineering, 44(3-4), pp. 269-282.

[52] Shiflett, M. B., and Yokozeki, A., 2005, "Solubilities and Diffusivities of Carbon Dioxide in Ionic Liquids: [Bmim][PF6] and [Bmim][BF4]," Industrial \& Engineering Chemistry Research, 44(12), pp. 4453-4464.

[53] Sheikha, H., Mehrotra, A. K., and Pooladi-Darvish, M., 2006, "An Inverse Solution Methodology for Estimating the Diffusion Coefficient of Gases in Athabasca Bitumen from Pressure-Decay Data," Journal of Petroleum Science and Engineering, 53(3-4), pp. 189-202. 
[54] Tharanivasan, A. K., Yang, C., and Gu, Y., 2006, "Measurements of Molecular Diffusion Coefficients of Carbon Dioxide, Methane, and Propane in Heavy Oil Under Reservoir Conditions," Energy Fuels, 20(6), pp. 2509-2517.

[55] Yang, C., and Gu, Y., 2006, "Diffusion Coefficients and Oil Swelling Factors of Carbon Dioxide, Methane, Ethane, Propane, and their Mixtures in Heavy Oil," Fluid Phase Equilibria, 243(1-2), pp. 64-73.

[56] Farajzadeh, R., Barati, A., Delil, H. A., 2007, "Mass Transfer of CO2 into Water and Surfactant Solutions," Petroleum Science and Technology, 25(12), pp. 14931511.

[57] Hou, Y., and Baltus, R. E., 2007, "Experimental Measurement of the Solubility and Diffusivity of $\mathrm{CO} 2$ in Room-Temperature Ionic Liquids using a Transient Thin-Liquid-Film Method," Industrial \& Engineering Chemistry Research, 46(24), pp. $8166-8175$.

[58] Rasmussen, M. L., and Civan, F., 2009, "Parameters of Gas Dissolution in Liquids obtained by Isothermal Pressure Decay," AIChE Journal, 55(1), pp. 9-23.

[59] Etminan, S. R., Maini, B. B., Chen, Z., 2010, "Constant-Pressure Technique for Gas Diffusivity and Solubility Measurements in Heavy Oil and Bitumen," Energy Fuels, 24(1), pp. 533-549.

[60] Moganty, S., and Baltus, R., 2010, "Diffusivity of Carbon Dioxide in RoomTemperature Ionic Liquids," Industrial \& Engineering Chemistry Research, 49(19), pp. 9370.

[61] Yokozeki, A., 2002, "Time-Dependent Behavior of Gas Absorption in Lubricant Oil," International Journal of Refrigeration, 25(6), pp. 695-704. 
[62] Gessner, T. R., and Barbosa Jr, J. R., 2006, "Modeling Absorption of Pure Refrigerants and Refrigerant Mixtures in Lubricant Oil," International Journal of Refrigeration, 29(5), pp. 773-780.

[63] Barbosa, J. R., and Ortolan, M. A., 2008, "Experimental and Theoretical Analysis of Refrigerant Absorption in Lubricant Oil," HVAC\&R Research, 14(1), pp. 141158.

[64] Marcelino Neto, M. A., and Barbosa Jr., J. R., 2011, "Absorption of Isobutane (R600a) in Lubricant Oil," Chemical Engineering Science, 66(9), pp. 1906-1915.

[65] Henni, A., Jaffer, S., and Mather, A. E., 1996, "Solubility of N2O and CO2 in NDodecane," The Canadian Journal of Chemical Engineering, 74(4), pp. 554-557.

[66] Hamblin, P., 1999, "Oxidative Stabilisation of Synthetic Fluids and Vegetable Oils," Journal of Synthetic Lubrication, 16(2), pp. 157-181.

[67] Adamczewska, J. Z., and Love, C., 2005, "Oxidative Stability of Lubricants Measured by PDSC CEC L-85-T-99 Test Procedure," Journal of Thermal Analysis and Calorimetry, 80(3), pp. 753-759.

[68] Maleville, X., Faure, D., Legros, A., and Hipeaux, J., 1996, "Oxidation of Mineral Base Oils of Petroleum Origin: The Relationship between Chemical Composition, Thickening, and Composition of Degradation Products," Lubrication Science, 9(1), pp. 1-60.

[69] Cerny, J., Strnad, Z., and Sebor, G., 2001, "Composition and Oxidation Stability of SAE 15W-40 Engine Oils," Tribology International, 34(2), pp. 127-134. 
[70] Erhan, S. Z., Sharma, B. K., and Perez, J. M., 2006, "Oxidation and Low Temperature Stability of Vegetable Oil-Based Lubricants," Industrial Crops and Products, 24(3), pp. 292-299.

[71] Grunberg, L., and Nissan, A. H., 1949, "Mixture Law for Viscosity," Nature, 164(4175), pp. 799-800.

[72] Totten, G. E., Westbrook, S. R., and Shah, R. J., 2003, "Fuels and Lubricants Handbook Technology, Properties, Performance, and Testing," ASTM International, West Conshokocken, PA.

[73] Larsson, R., and Andersson, O., 2000, "Lubricant Thermal Conductivity and Heat Capacity Under High Pressure," Proceedings of the Institution of Mechanical Engineers, Part J: Journal of Engineering Tribology, 214(4), pp. 337-342.

[74] Jensen, M., and Jackman, D., 1984, "Prediction of Nucleate Pool Boiling HeatTransfer Coefficients of Refrigerant-Oil Mixtures," Journal of Heat Transfer, 106(1), pp. 184-190.

[75] Jeng, Y., Chang, C., and Wang, C., 2001, "Vapor Pressure of R-410A/Oil and R407C/Oil Mixtures," Applied Thermal Engineering, 21(8), pp. 863-870.

[76] He, M., 2003, "Thermoelastohydrodynamic Analysis of Fluid Film Journal Bearings," Ph.D. Thesis, University of Virginia, Charlottesville, VA.

[77] He, M., and Allaire, P., 2002, "Thermoelastohydro-dynamic analysis of journal bearings with 2D generalized energy equation," Proceedings of the 6th International Conference on Rotor Dynamics, IFTOMM, Sydney, Australia. 
[78] He, M., Allaire, P., Barrett, L., and Nicholas, J., 2002, "TEHD modeling of leading edge groove tilting pad bearings," Proceedings of the 6th International Conference on Rotor Dynamics, IFTOMM, Sydney, Australia.

[79] Weaver, B., Younan, A., Dimond, T., Wang, Z., Allaire, P., and Clarens, A., 2013, "Properties and Performance of Gas-Expanded Lubricants in Tilting Pad Journal Bearings," Tribology Transactions, 56(4), pp. 687-696.

[80] Taniguchi, S., Makino, T., and Takeshita, K., 1990, "A Thermohydrodynamic Analysis of Large Tilting-Pad Journal Bearing in Laminar and Turbulent Flow Regimes with Mixing," Journal of Tribology, 112(3), pp. 542-548.

[81] Ng, C., and Pan, C., 1965, "A Linearized Turbulent Lubrication Theory," Journal of Basic Engineering, 87, pp. 675-682.

[82] Elrod Jr, H., and Ng, C., 1967, "A Theory for Turbulent Fluid Films and its Application to Bearings," Journal of Lubrication Technology, 89(3), pp. 346-362.

[83] Petroff, N., 1883, "Friction in Machines and the Effect of the Lubricant," Inzh. Zh., 1, pp. 71-104.

[84] Ferrando, N., Mougin, P., and Defiolle, D., 2008, "Solubility and Diffusion Coefficient of Hydrogen Sulphide in Polyethylene Glycol 400 from 100 to 140C," Oil and Gas Science and Technology, 63(3), pp. 343-351.

[85] Martin, F. A., and Garner, D. R., 1974, "Plain Journal Bearings Under Steady Loads: Design Guidance for Safe Operations," First European Tribology Conference, pp. 1-16. 
[86] Glavatskih, S. B., and Larsson, R., 2003, "Performance of Synthetic Oils in the Hydrodynamic Regime-1. Experimental," Journal of Synthetic Lubrication, 20(1), pp. 15-24.

[87] Flack, R., Lanes, R., and Gambel, P., 1981, "Effects of Lubricant Viscosity on the Experimental Response of a Three-Mass Flexible Rotor in Two Types of Journal Bearings," Wear, 67(2), pp. 201-216.

[88] Singhal, S., 2004, "A Simplified Thermohydro-dynamic Stability Analysis of the Plain Cylindrical Hydrodynamic Journal Bearings,” M.S. Thesis, Louisiana State University, Baton Rouge, LA.

[89] Roach, M., and Goodwin, M., 1992, "Vibration Control in Rotating Machinery by the use of Accumulators or Aerated Lubricants," Proceedings of the International Conference on Rotating Machine Dynamics, Springer, London, England, pp. 367375.

[90] Samantaray, A., Bhattacharyya, R., and Mukherjee, A., 2006, "An Investigation into the Physics Behind the Stabilizing Effects of Two-Phase Lubricants in Journal Bearings," Journal of Vibration and Control, 12(4), pp. 425-442.

[91] Swamy, S., Prabhu, B., and Rao, B., 1977, "Steady State and Stability Characteristics of a Hydrodynamic Journal Bearing with a Non-Newtonian Lubricant," Wear, 42(2), pp. 229-244.

[92] Raghunandana, K., and Majumdar, B. C., 1999, "Stability of Journal Bearing Systems using Non-Newtonian Lubricants: A Non-Linear Transient Analysis," Tribology International, 32(4), pp. 179-184. 
[93] Williamson, B. P., Walters, K., Bates, T. W., Coy, R. C., and Milton, A. L., 1997, "The Viscoelastic Properties of Multigrade Oils and their Effect on JournalBearing Characteristics," Journal of Non-Newtonian Fluid Mechanics, 73(1-2), pp. $115-126$.

[94] Li, X. K., Gwynllyw, D. R., Davies, A. R., and Phillips, T. N., 2000, "On the Influence of Lubricant Properties on the Dynamics of Two-Dimensional Journal Bearings," Journal of Non-Newtonian Fluid Mechanics, 93(1), pp. 29-59.

[95] Barrett, L. E., 1978, "Stability and Nonlinear Response of Rotor-Bearing Systems with Squeeze Film Bearings," Ph.D. Thesis, University of Virginia, Charlottesville, Virginia.

[96] Wagner, M. B., 2011, “Model Reduction Methods for Rotordynamic Analysis: An Exploration, Comparison, and Metrics," Ph.D. Thesis, University of Virginia, Charlottesville, VA.

[97] Nicholas, J., Gunter, E., and Barrett, L., 1978, "The Influence of Tilting Pad Bearing Characteristics on the Stability of High Speed Rotor-Bearing Systems," Proceedings of the Design Engineering Conference, Topics in Fluid Film Bearing and Rotor Bearing System Design and Optimization, pp. 55-78.

[98] API, 2005, "API Standard Paragraphs Rotordynamic Tutorial: Laterial Critical Speeds, Unbalance Response, Stability, Train Torsionals, and Rotor Balancing," 2nd ed., American Petroleum Institute, API Standard No. 684, Washington, DC.

[99] API, 2002, "Axial and Centrifugal Compressors and Expander-compressors for Petroleum, Chemical, and Gas Industry Service, Downstream Service," 7th ed., American Petroleum Institute, API Standard No. 617, Washington, DC. 
[100] Stringer, D. B., 2009, "Geared Rotor Dynamic Methodologies for Advancing Prognostic Modeling Capabilities in Rotary-Wing Transmission Systems," Ph.D. Thesis, University of Virginia, Charlottesville, VA.

[101] Kaplan, J. A., Dousti, S., Allaire, P.E., Nichols, B., Dimond, T., and Untaroiu, A., 2013, "Dynamic Modeling of Gears and Geared Systems," Proceedings of ASME Turbo Expo 2013: Power for Land, Sea, and Air, GT2013, Paper No. GT201394654, June 3-7 2013, San Antonio, TX.

[102] Cao, J., 2012, "Transient Analysis of Flexible Rotors with Nonlinear Bearings, Dampers and External Forces," Ph.D. Thesis, University of Virginia, Charlottesville, VA.

[103] Untaroiu, A., Hayrapetian, V., Untaroiu, C. D., Wood, H. G., Schiavello, B., and McGuire, J., 2013, "On the Dynamic properties of pump liquid seals," ASME Journal of Fluids Engineering, 135(5), pp. 051104-1-051104-10.

[104] Muszynska, A., and Goldman, P., 1995, "Chaotic Responses of Unbalanced Rotor/Bearing/Stator Systems with Looseness Or Rubs," Chaos, Solitons \& Fractals, 5(9), pp. 1683-1704.

[105] Huang, Z., Zhou, J., Yang, M., and Zhang, Y., 2011, "Vibration Characteristics of a Hydraulic Generator Unit Rotor System with Parallel Misalignment and RubImpact," Archive of Applied Mechanics, 81(7), pp. 829-838.

[106] Sinha, S. K., 2005, "Non-Linear Dynamic Response of a Rotating Radial Timoshenko Beam with Periodic Pulse Loading at the Free-End," International Journal of Non-Linear Mechanics, 40(1), pp. 113-149. 
[107] Cao, D., Gong, X., Wei, D., Chu, S., and Wang, L., 2011, "Nonlinear Vibration Characteristics of a Flexible Blade with Friction Damping due to Tip-Rub," Shock and Vibration, 18(1-2), pp. 105-114.

[108] Khanlo, H., Ghayour, M., and Ziaei-Rad, S., 2011, "Chaotic Vibration Analysis of Rotating, Flexible, Continuous Shaft-Disk System with a Rub-Impact between the Disk and the Stator," Communications in Nonlinear Science and Numerical Simulation, 16(1), pp. 566-582.

[109] Khanlo, H., Ghayour, M., and Ziaei-Rad, S., 2012, "Bearings Coefficients Effects on Chaotic and Bifurcation Behavior of Flexible Rotor Systems Subjected to RubImpact." Journal of Vibroengineering, 14(2), pp. 920-942.

[110] Wang, J., Zhou, J., Dong, D., Yan, B., and Huang, C., 2013, "Nonlinear Dynamic Analysis of a Rub-Impact Rotor Supported by Oil Film Bearings," Archive of Applied Mechanics, 83(3), pp. 413-430.

[111] Zhang, Y., and Wang, W., 2010, "The Nonlinear Dynamic Behavior of DoubleDisk Isotropic Rotor System with Rub-Impact," Applied Mechanics and Materials, 34-35, pp. 1483-1487.

[112] Wang, W., Gao, J., Zhang, Y., and Yao, J., 2011, "Numerical and Experimental Investigation on the Controlling for Rotor-to-Stationary Part Rubbing in Rotating Machinery," ASME 2011 Turbo Expo: Turbine Technical Conference and Exposition, American Society of Mechanical Engineers, Vancouver, Canada, pp. 425-434. 
[113] Chang-Jian, C., and Chen, C., 2006, "Nonlinear Dynamic Analysis of a Flexible Rotor Supported by Micropolar Fluid Film Journal Bearings," International Journal of Engineering Science, 44(15), pp. 1050-1070.

[114] Chang-Jian, C., and Chen, C., 2009, "Chaotic Response and Bifurcation Analysis of a Flexible Rotor Supported by Porous and Non-Porous Bearings with Nonlinear Suspension," Nonlinear Analysis: Real World Applications, 10(2), pp. 1114-1138.

[115] Wang, C., 2010, "Bifurcation and Nonlinear Analysis of a Flexible Rotor Supported by a Relative Short Spherical Gas Bearing System," Communications in Nonlinear Science and Numerical Simulation, 15(9), pp. 2659-2671.

[116] Weaver, B., Dimond, T., Kaplan, J., Untaroiu, A., and Clarens, A., 2014, "GasExpanded Lubricant Performance and Effects on Rotor Stability in Turbomachinery," Proceedings of the ASME Turbo Expo 2014: Turbine Technical Conference and Exposition, Paper No. GT2014-26980, June 16-20, 2014, Düsseldorf, Germany, pp. 1-13.

[117] Oberg, E., Jones, F., Ryffel, H., McCauley, C., and Heald, R., 2008, "Machinery’s Handbook 28th Edition," Industrial Press.

[118] Allaire, P. E., and Kocur, J., 1985, "Oil Seal Effects and Subsynchronous Vibrations in High-Speed Compressors," NASA, Lewis Research Center Instability in Rotating Machinery (SEE N 86-30160 21-37), pp. 205-223.

[119] Warring, R.H., 1981, "Seals and Sealing Handbook," Trade \& Technical Press.

[120] Hirs, G., 1973, "A Bulk-Flow Theory for Turbulence in Lubricant Films," Journal of Tribology, 95(2), pp. 137-145. 
[121] Allaire, P., Lee, C., and Gunter, E., 1978, "Dynamics of Short Eccentric Plain Seals with High Axial Reynolds Number," Journal of Spacecraft and Rockets, 15(6), pp. 341-347.

[122] Wen, C., Yang, A., Tseng, L., and Tsai, W., 2011, "Flow Analysis of a Ribbed Helix Lip Seal with Consideration of Fluid-structure Interaction," Computers \& Fluids, 40(1), pp. 324-332.

[123] Kalsi, M., Conroy, W., and Dietle, L., 1997, "A novel high-pressure rotary shaft seal facilitates innovations in drilling and production equipment," SPE/IADC Drilling Conference, pp. 469-477.

[124] Picardo, A., and Childs, D. W., 2005, "Rotordynamic Coefficients for a Tooth-onStator Labyrinth Seal at 70 Bar Supply Pressures: Measurements Versus Theory and Comparisons to a Hole-Pattern Stator Seal," Journal of Engineering for Gas Turbines and Power, 127(4), pp. 843-855.

[125] Zhang, W. F., Yang, J. G., and Cao, H., 2011, "Experimental Identification of Fluid-Induced Force in Labyrinth Seals," Journal of Mechanical Science and Technology, 25(10), pp. 2485-2494.

[126] Li, Z., Li, J., Yan, X., and Feng, Z., 2011, "Effects of Pressure Ratio and Rotational Speed on Leakage Flow and Cavity Pressure in the Staggered Labyrinth Seal," Journal of Engineering for Gas Turbines and Power, 133(11), pp. 114503.

[127] Asok, S., Sankaranarayanasamy, K., Sundararajan, T., Vaidyanathan, G., and Kumar, K., 2011, "Pressure Drop and Cavitation Investigations on Static HelicalGrooved Square, Triangular and Curved Cavity Liquid Labyrinth Seals," Nuclear Engineering and Design, 241(3), pp. 843-853. 
[128] Kocur, J. A., Nicholas, J. C., and Lee, C. C., 2007, "Surveying tilting pad journal bearing and gas labyrinth seal coefficients and their effect on rotor stability," 36th Turbomachinery Symposium, Turbomachinery Laboratory, Texas A\&M University, College Station, TX, pp. 10-13.

[129] Zhao, W., Nielsen, T., and Billdal, J., 2010, "Effects of cavity on leakage loss in straight-through labyrinth seals," IOP Conference Series: Earth and Environmental Science, IOP Publishing, 12(1), pp. 012002.

[130] Chupp, R. E., Hendricks, R. C., Lattime, S. B., 2006, "Sealing in Turbomachinery," Journal of Propulsion and Power, 22(2), pp. 313-349.

[131] Ross, S. L., and Beckinger, R. F., 2003, "Compressor Seal Selection and Justification," Proceedings of the Thirty-second Turbomachinery Symposium, Turbomachinery Laboratory, Texas A\&M University, College Station, Texas, pp. $167-177$.

[132] Liu, P., Ou, Q. P., Zhou, H. B., and Li, M., 2011, "Development and Application of Non-Contact Seal Assembly for High-Speed Shaft Ends," Applied Mechanics and Materials, 86, pp. 846-849.

[133] Proctor, M. P., and Delgado, I. R., 2004, "Leakage and power loss test results for competing turbine engine seals," ASME Turbo Expo 2004: Power for Land, Sea, and Air, American Society of Mechanical Engineers, pp. 441-451.

[134] Delgado, I. R., and Proctor, M. P., 2006, "Continued Investigation of Leakage and Power Loss Test Results for Competing Turbine Engine Seals," Paper No. NASA/TM-2006-214420. 
[135] Childs, D. W., Shin, Y., and Seifert, B., 2008, "A Design to Improve the Effective Damping Characteristics of Hole-Pattern-Stator Annular Gas Seals," Journal of Engineering for Gas Turbines and Power, 130(1), pp. 012505.

[136] Arghir, M., Zerarka, A., and Pineau, G., 2011, "Rotordynamic Analysis of Textured Annular Seals with Multiphase (Bubbly) Flow," Incas Bulletin, 3(3), pp. $3-13$.

[137] Gowda, D. R., and Prabhu, B., 2002, "High-Pressure Annular Seal Leakage and Rotordynamics with Application to Turbomachinery," International Journal of Rotating Machinery, 8(6), pp. 403-411.

[138] Childs, D. W., Graviss, M., and Rodriguez, L. E., 2007, "Influence of Groove Size on the Static and Rotordynamic Characteristics of Short, Laminar-Flow Annular Seals," Journal of Tribology, 129(2), pp. 398-406.

[139] San Andrés, L., 2012, "Rotordynamic Force Coefficients of Bubbly Mixture Annular Pressure Seals," Journal of Engineering for Gas Turbines and Power, 134(2), pp. 022503.

[140] Childs, D. W., and Wade, J., 2004, "Rotordynamic-Coefficient and Leakage Characteristics for Hole-Pattern-Stator Annular Gas Seals-Measurements Versus Predictions," Journal of Tribology, 126(2), pp. 326-333.

[141] Childs, D. W., Rodriguez, L. E., Cullotta, V., Al-Ghasem, A., and Graviss, M., 2006, "Rotordynamic-Coefficients and Static (Equilibrium Loci and Leakage) Characteristics for Short, Laminar-Flow Annular Seals," Journal of Tribology, 128(2), pp. 378-387. 
[142] Ertas, B. H., Delgado, A., and Vannini, G., 2012, "Rotordynamic Force Coefficients for Three Types of Annular Gas Seals with Inlet Preswirl and High Differential Pressure Ratio," Journal of Engineering for Gas Turbines and Power, 134(4), pp. 042503.

[143] Childs, D.W., Arthur, S., and Mehta, N.J., 2013, "The Impact of Hole Depth on the Rotordynamic and Leakage Characteristics of Hole-Pattern-Stator Gas Annular Seals," Proceedings of the ASME Turbo Expo 2013: Turbine Technical Conference and Exposition, Paper No. GT2013-94333, June 3-7, 2013, San Antonio, Texas, pp. 175-190.

[144] Oike, M., Nosaka, M., Kikuchi, M., and Hasegawa, S., 1999, "Two-Phase Flow in Floating-Ring Seals for Cryogenic Turbopumps," Tribology Transactions, 42(2), pp. 273-281.

[145] Lee, Y., Shin, S., Ryu, K., Kim, C., and Jang, G., 2005, "Test Results for Leakage and Rotordynamic Coefficients of Floating Ring Seals in a High-Pressure, HighSpeed Turbopump," Tribology Transactions, 48(3), pp. 273-282.

[146] Ha, T., Lee, Y., and Kim, C., 2002, "Leakage and Rotordynamic Analysis of a High Pressure Floating Ring Seal in the Turbo Pump Unit of a Liquid Rocket Engine," Tribology International, 35(3), pp. 153-161.

[147] Ketola, H. N., and McGrew, J., 1967, "Turbulent Operation of the Visco Seal," ASLE Transactions, 10(3), pp. 256-272.

[148] King, A. E., 1965, "Screw Type Shaft Seals for Potassium Lubricated Generators," IEEE Transactions On Aerospace, 2, pp. 471-479. 
[149] Necker, N., 2002, "Mechanical sealing technology used in multiphase production," Canadian International Petroleum Conference, Petroleum Society of Canada.

[150] Necker, N., 2005, "Mechanical Sealing Technology used in Multiphase Pumpingthe Benefits," World Pumps, 467, pp. 20-22.

[151] Wang, Y., Yang, H., Wang, Y., Duan, X., and Wang, H., 2005, "Experimental Investigations and Field Applications of Oil-Film-Lubricated Mechanical Face Seals with Spiral Grooves," Tribology Transactions, 48(4), pp. 589-596.

[152] Goilkar, S. S., and Hirani, H., 2008, "Design and Development of a Test Setup for Online Wear Monitoring of Mechanical Face Seals using a Torque Sensor," Tribology Transactions, 52(1), pp. 47-58.

[153] Yu, X. Q., Liu, M. H., Wang, Z. H., Peng, P. Y., and Cai, R. L., 2006, "Experimental investigation on friction performance of mechanical seals with a laser-textured seal face," Materials Science Forum, 532, pp. 81-84.

[154] Mammadov, V. A., Yusifov, A., and Tacon, K. P., 2010, "Upstream Pumping Technogoly In Centrifugal Pump Mechanical Sealing Applications-Field Experience With High Duty Sea Water Injection Pumps," Proceedings of the Twenty-Sixth International Pump Users Symposium.

[155] Shapiro, W., Walowit, J., and Jones, H. F., 1984, "Analysis of Spiral-Groove Face Seals for Liquid Oxygen," ASLE Transactions, 27(3), pp. 177-188.

[156] Zirkelback, N., 2000, "Parametric Study of Spiral Groove Gas Face Seals," Tribology Transactions, 43(2), pp. 337-343. 
[157] Liu, Z., Liu, Y., and Liu, X., 2007, "Optimization Design of Main Parameters for Double Spiral Grooves Face Seal," Science in China Series E: Technological Sciences, 50(4), pp. 448-453.

[158] Pennink, H., 1985, "The Gas Lubricated Spiral Grooved Face Seal in the Process Industry," Dynamics, 1(2), pp. 3-4.

[159] Zhang, G., Zhao, W., Yan, X., and Yuan, X., 2011, "A Theoretical and Experimental Study on Characteristics of Water-Lubricated Double SpiralGrooved Seals," Tribology Transactions, 54(3), pp. 362-369.

[160] Shah, P., 1988, "Dry Gas Compressor Seals," Proceedings of the Seventeenth Turbomachinery Symposium.

[161] Brandt, J., Müller-Link, D., and Rohlfing, G., 2000, "Leakage is Management by a New Sealing System for Twin-Screw Multiphase Pumps," Abu Dhabi International Petroleum Exhibition and Conference, Society of Petroleum Engineers.

[162] Evans, J. G., and Janssen, S., 2002, "Developments in Sealing Technology Within Multiphase Pumps," Nineteenth International Pump Users Symposium, Turbomachinery Laboratory, Texas A\&M University, College Station, Texas, pp. 25-32.

[163] Müller, H., 1972, "An Automatic Barrier Seal: Theory and Experiment," ASLE Transactions, 15(4), pp. 288-295.

[164] Untaroiu, A., Migliorini, P., Wood, H. G., Allaire, P. E., and Kocur, J. A., 2009, "Hole-Pattern Seals: A Three Dimensional CFD Approach for Computing Rotordynamic Coefficient and Leakage Characteristics," ASME 2009 International 
Mechanical Engineering Congress and Exposition, American Society of Mechanical Engineers, pp. 981-990.

[165] Migliorini, P. J., Untaroiu, A., Wood, H. G., and Allaire, P. E., 2012, "A Computational Fluid Dynamics/Bulk-Flow Hybrid Method for Determining Rotordynamic Coefficients of Annular Gas Seals," Journal of Tribology, 134(2), pp. 022202.

[166] Untaroiu, A., Liu, C., Migliorini, P. J., Wood, H. G., and Untaroiu, C. D., 2014, "Hole-Pattern Seals Performance Evaluation using Computational Fluid Dynamics and Design of Experiment Techniques," Journal of Engineering for Gas Turbines and Power, 136(10), pp. 102501.

[167] Witt, W. C., Migliorini, P. J., Untaroiu, A., and Wood, H. G., "A Hybrid CFD/Bulk-Flow Method For Analyzing Incompressible Flow In Labyrinth Seals," Progress Report, University of Virginia.

[168] Migliorini, P. J., Untaroiu, A., Witt, W. C., Morgan, N. R., and Wood, H. G., 2014, "Hybrid Analysis of Gas Annular Seals with Energy Equation," Journal of Tribology, 136(3), pp. 031704.

[169] Untaroiu, A., Liu, C., Migliorini, P. J., Wood, H. G., and Untaroiu, C. D., 2013, "Hole-Pattern Seals Performance Optimization using Computational Fluid Dynamics and Design of Experiment Techniques," ASME 2013 International Mechanical Engineering Congress and Exposition, American Society of Mechanical Engineers, pp. V07BT08A058-V07BT08A058.

[170] Weaver, B. K., Zhang, Y., Clarens, A. F., and Untaroiu, A., November 8-13, 2014, "Nonlinear Analysis of Rub Impact in a Three-Disk Rotor and Correction via 
Bearing and Lubricant Adjustment," Proceedings of the ASME 2014 International Mechanical Engineering Congress \& Exposition, Paper No. IMECE201-40055, November 8-13, 2014, Montreal, Quebec, Canada. 